\title{
Polarity and Competition in the Development of the Calyx of Held Terminal in the Medial Nucleus of the Trapezoid Body in the Mouse
}

\author{
Paul Steven Holcomb \\ West Virginia University, pholcomb@mix.wvu.edu
}

Follow this and additional works at: https://researchrepository.wvu.edu/etd

Part of the Computational Neuroscience Commons, and the Developmental Neuroscience Commons

\section{Recommended Citation \\ Holcomb, Paul Steven, "Polarity and Competition in the Development of the Calyx of Held Terminal in the Medial Nucleus of the Trapezoid Body in the Mouse" (2018). Graduate Theses, Dissertations, and Problem Reports. 3703. \\ https://researchrepository.wvu.edu/etd/3703}

This Dissertation is protected by copyright and/or related rights. It has been brought to you by the The Research Repository @ WVU with permission from the rights-holder(s). You are free to use this Dissertation in any way that is permitted by the copyright and related rights legislation that applies to your use. For other uses you must obtain permission from the rights-holder(s) directly, unless additional rights are indicated by a Creative Commons license in the record and/ or on the work itself. This Dissertation has been accepted for inclusion in WVU Graduate Theses, Dissertations, and Problem Reports collection by an authorized administrator of The Research Repository @ WVU.

For more information, please contact researchrepository@mail.wvu.edu. 


\title{
Polarity and Competition in the Development of the Calyx of Held Terminal in the Medial Nucleus of the Trapezoid Body in the Mouse
}

\author{
Paul Steven Holcomb \\ Dissertation submitted \\ to the Rockefeller Neuroscience Institute \\ at West Virginia University
}

in partial fulfillment of the requirements for the degree of

Doctor of Philosophy in
Neuroscience

George Spirou, Ph.D., Chair

Peter Mathers, Ph.D

Eric Tucker, Ph.D.

Visvanathan Ramamurthy, Ph.D.

Sergiy Yakovenko, Ph.D.

Department of Neuroscience

Morgantown, West Virginia

2018

Keywords: neuron, development, synapse, polarity, MNTB, calyx

Copyright 2018 Paul Steven Holcomb 


\title{
ABSTRACT
}

\section{Polarity and Competition in the Development of the Calyx of Held Terminal in the Medial Nucleus of the Trapezoid Body in the Mouse}

\author{
Paul Steven Holcomb
}

In the auditory brainstem, the connection between globular bushy cells of the anteroventral cochlear nucleus and principal cells (PCs) of the medial nucleus of the trapezoid body (MNTB) is created by one of the largest nerve terminals in the central nervous system, the calyx of Held $(\mathrm{CH})$. The characteristics of the $\mathrm{CH}$ :MNTB connection-a short developmental period (48-72 hours), accessibility for recording from pre- and postsynaptic components, and clear monoinnervated end point-make this system an ideal model system for studying nervous system development. Model systems undergo stereotyped stages of development, including exuberant overinnervation, competition between terminals, and a refinement of innervation through removal of weak inputs. However, unlike other similar model systems (climbing fiber:Purkinje cell, retinal ganglion cells:dorsolateral geniculate nucleus), it has been a longstanding question whether the $\mathrm{CH}$ :MNTB system undergoes competition. We investigated the innervation state of PCs using the novel technique of segmentation and 3D reconstruction of PCs and their associated inputs across important developmental timepoints (postnatal days (P)2,3,4,6,9,30). This was accomplished by application of serial block-face scanning electron microscopy (SBEM), a method of serial section electron microscopy providing high spatial resolution ( 4-10nm) and a high degree of alignment between images with very little section loss. Applying this technique, we show early exuberant innervation of PCs (P2), establish that competition is a common process, and pinpoint the 24-hour period from P3-P4 as a uniquely active day in $\mathrm{CH}$ growth during which terminal contact with $\mathrm{PCs}$ increases at a rate exceeding $200 \mathrm{\mu m}^{2} /$ day. Common morphological characteristics of the $\mathrm{CH}$ :MNTB connection also became qualitatively evident based on $3 \mathrm{D}$ reconstructions, particularly an eccentric $\mathrm{PC}$ nucleus and preference for polarized terminal growth. Based on these observations, we undertook a quantitative study of polarity in $\mathrm{CH}$ :MNTB development using our 3D reconstructions. The results of this investigation demonstrate a novel polarity in development of both the $\mathrm{CH}$ and $\mathrm{PC}$; developing PCs are characterized by eccentrically placed nuclei that establish an "intrasomatic polarity" that persists through young adulthood (P30). This polarity appears to define a unique territory opposite of the nuclear location that is amenable to growth of the calyx, is enriched in dendrites, and is selectively enlarged as the principal cell matures to create glia-free surface area for innervation. To our knowledge, this is the first report of a polarity program in the coordinated pre- and postsynaptic development of a non-laminar brain region. Additionally, our findings have codified a progression of dendritic pruning in the maturation of principal cells that may influence and be influenced by the developmental state of the cell. Taken in totality, these results indicate a highly polarized, systemic competitive process in the MNTB during the development of the calyx of Held and suggests potential mediators of competition that deserve further study. 


\section{Dedication:}

For my wife, Micky, who said "It's your turn."

and

For my children, Colin and Isabel.

I look forward to being "Dr. Daddy". 


\section{Acknowledgements:}

Thank you to my advisor, Dr. George Spirou, for all of the time, effort, and patience he has put into helping me get this far; I won't let you down.

Thank you to my committee for the advice, guidance, and sometimes tough love you've shown me. You've made this dissertation-and me-better for it.

Thank you to my lab mates, both past and present, for being a sounding board for ideas and for pitching in to bring our electron microscopy images to life in 3D.

Thank you to more than 30 undergraduate and graduate student tracers, without whom this research would not have been possible. Your time and perseverance have created some amazing cells!

Thank you to Brian Pope, whose skill with tissue processing made possible the beautiful electron microscopy images I had to work with.

Thank you to Dr. Gerry Hobbs, who helped me figure out whether all these curves meant something. You helped me find an unexpected interest in statistics that will serve me well.

Thank you to my family who have supported and encouraged me throughout this entire process. I love and cherish all of you. 


\section{Table of Contents}

Chapter 1: Introduction and Literature Review ........................................................ 1

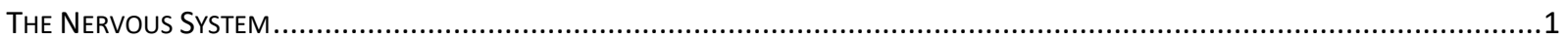

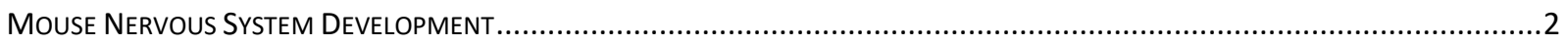

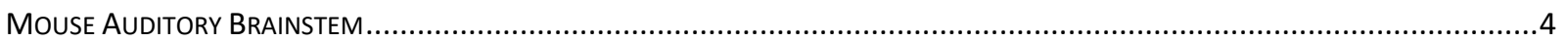

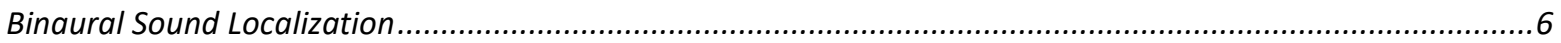

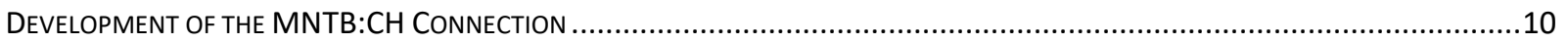

Cellular Development

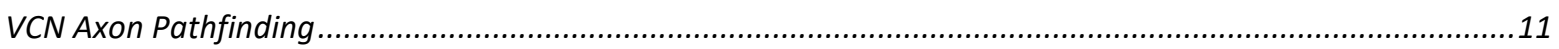

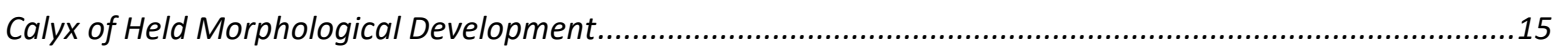

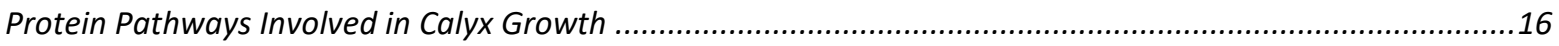

Development of Electrophysiological Characteristics of the Calyx of Held .................................................23

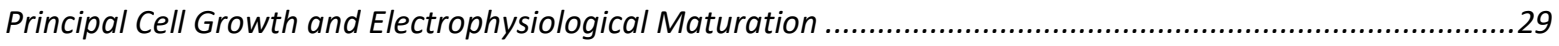

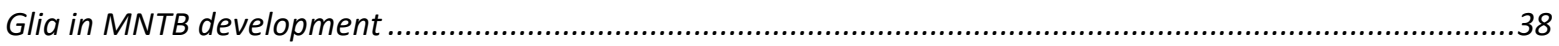

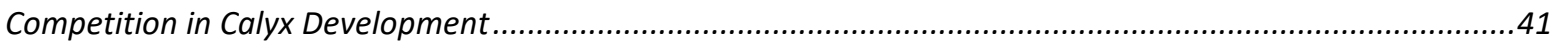

Chapter 2: Synaptic Inputs Compete During Rapid Formation of the Calyx of Held: A New Model System for Neural Development ......................................................................47

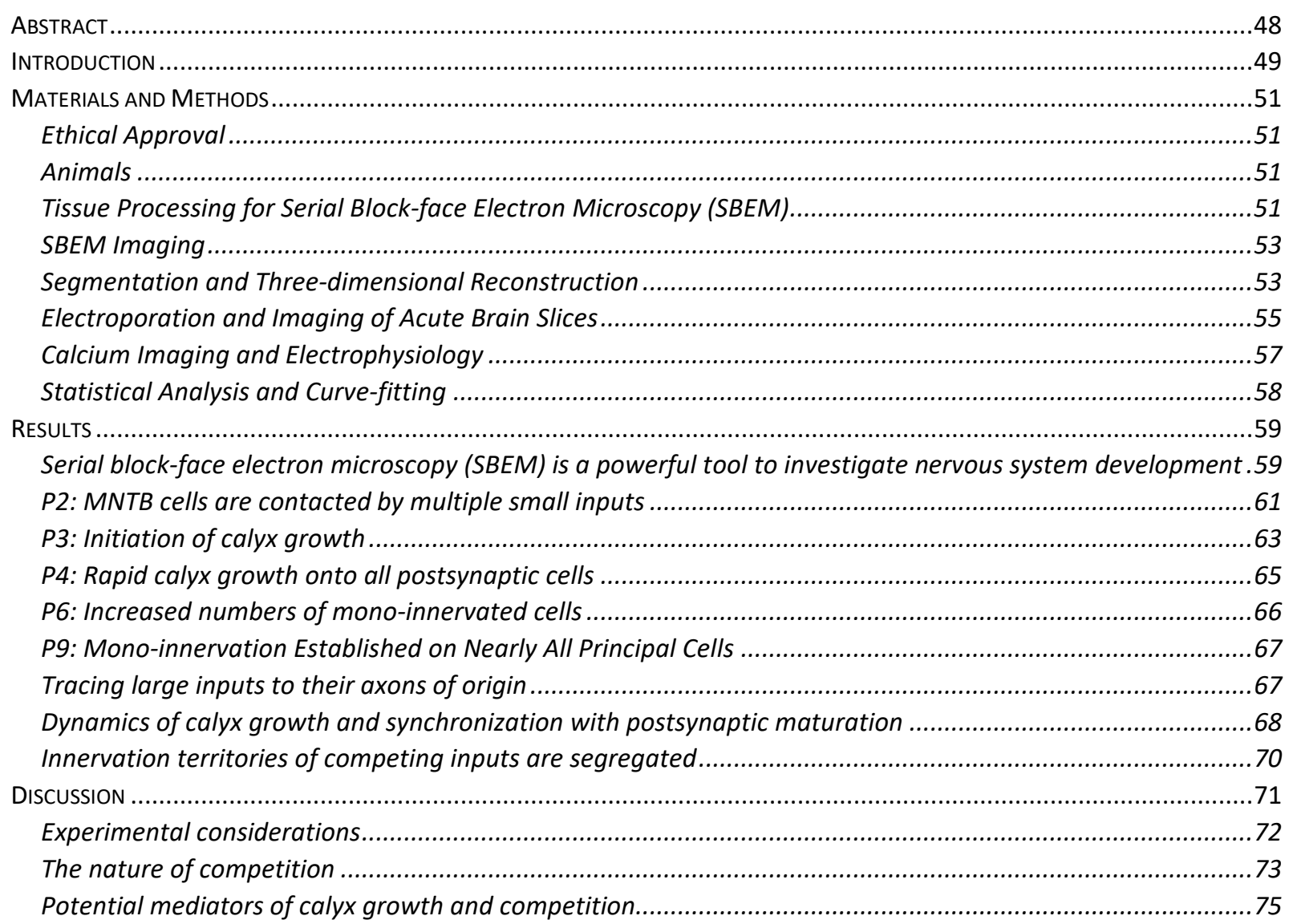

Chapter 3: Terminal Growth Occurs Asymmetrically with Respect to Nuclear Position during Development of the Calyx of Held in the Mouse ................................................77 


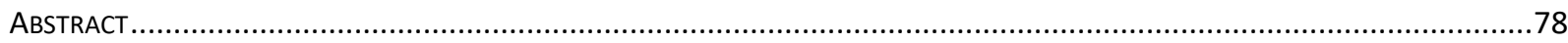

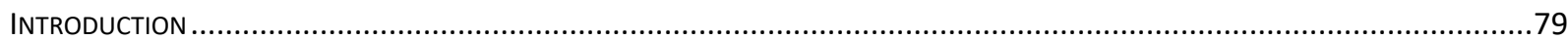

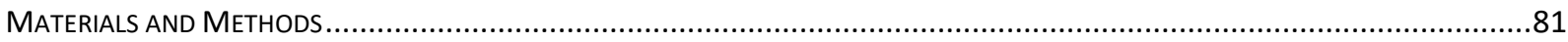

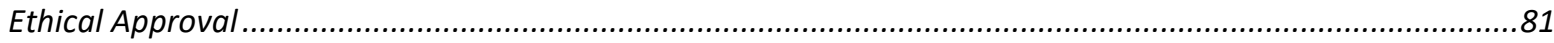

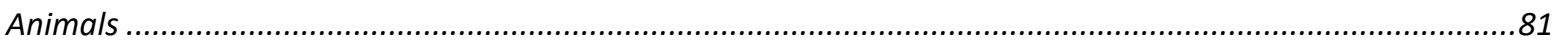

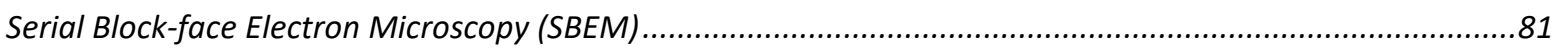

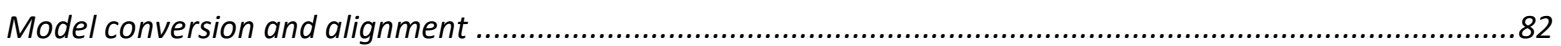

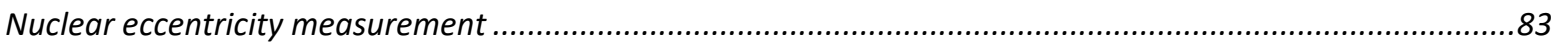

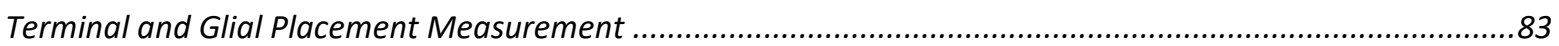

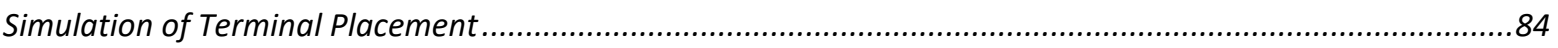

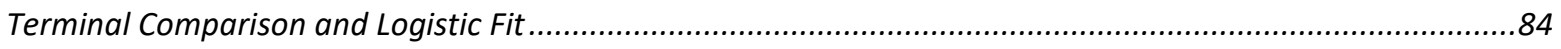

Poisson Reconstruction and Somatic Spicule Surface Area Quantification.................................................8

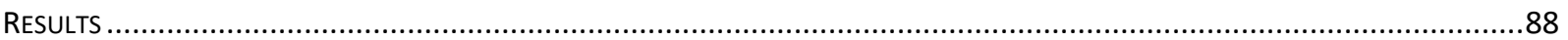

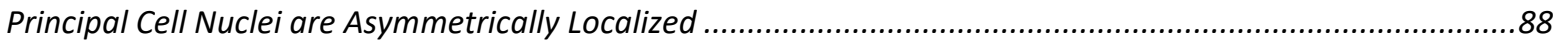

Terminal Location is Polarized with Respect to Nuclear Position....................................................................92

Membrane is Added Asymmetrically During Principal Cell Growth ..............................................................94

Glial Apposition Restricts Innervation from the Nuclear Pole ...................................................................97

Principal Cell Axon and Dendrite Placement is Oppositional and Biased Toward the Cytoplasmic Pole ............98

Dendrites Guide Calycigenic Axons Toward the Principal Cell Soma ........................................................100

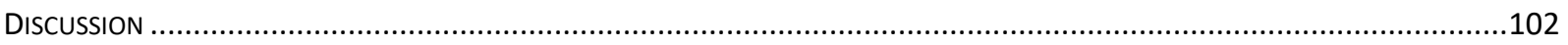

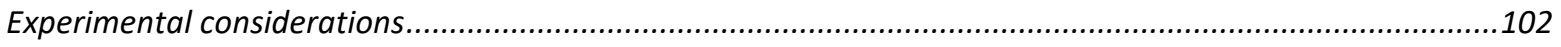

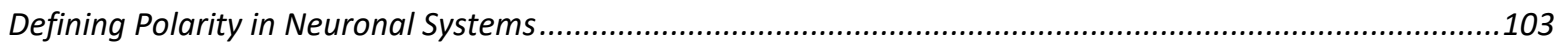

Polarity in Terminal Formation: Similarities to the Climbing Fiber:Purkinje Cell System ..............................104

Functional Consequences of Nuclear Eccentricity ..................................................................................106

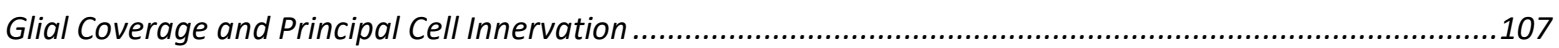

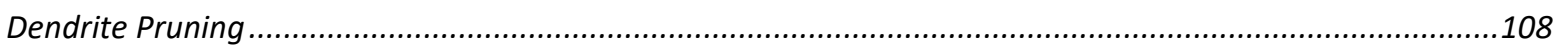

Chapter 4: Discussion and Future Directions .............................................................110

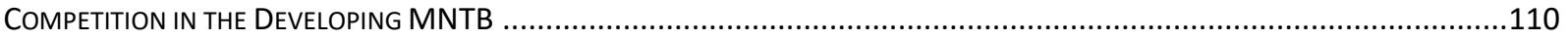

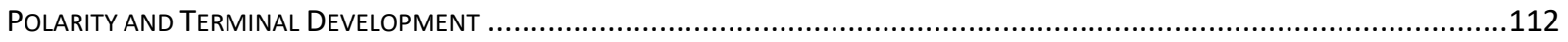

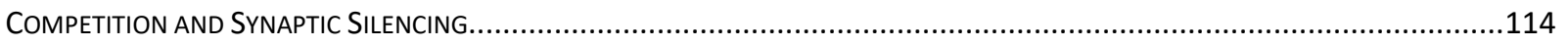

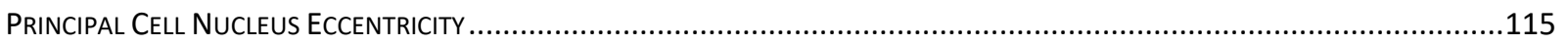

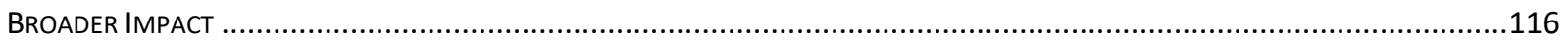

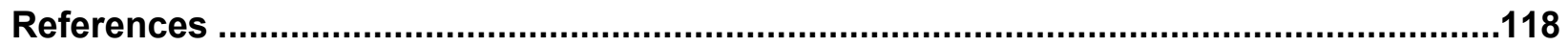




\section{List of Figures and Tables}

Figure 1: Development of the Nervous System

Figure 2: Rhombomeres of the Mouse Hindbrain.

Figure 3: Cochlear Nucleus Anatomy.

Figure 4: Auditory Brainstem Anatomy.

Figure 5: Connectivity of Auditory Nuclei for Binaural Sound Localization.

Figure 6: Embryonic Origins of the Anteroventral Cochlear Nucleus (AVCN).

Figure 7: Embryonic Origins of the Medial Nucleus of the Trapezoid Body (MNTB).

Figure 8: VCN Axon Guidance is Mediated by DCC-Netrin and Slit-Robo Binding.

Figure 9: Eph-ephrin Binding Inhibits Ipsilateral Calyx Formation.

Figure 10: Morphological Development of the Calyx of Held.

Figure 11: Protein Pathways Involved in Calyx Development.

Figure 12: Spontaneous Activity in the Pre-hearing Auditory System.

Figure 13: Changes in Calyceal $\mathrm{Na}+$ and $\mathrm{K}+$ Channels Sharpen the Presynaptic Action

Potential.

Figure 14: Changes in Voltage Gated Calcium Channels (VGCCs) during Calyx Development.

Figure 15: Morphological Changes in the Developing Principal Cell of the MNTB.

Figure 16: Electrophysiological Changes in the Developing Principal Cell of the MNTB.

Figure 17: Maturation of Principal Cell Electrophysiology is Mediated in Part by Potassium

Channels.

Figure 18: Changes in Principal Cell NMDA Channels During Development.

Figure 19: Changes in Principal Cell AMPA Channels During Development.

Figure 20: Glial Development and Association with the CoH:MNTB Connection in MNTB.

Figure 21: Competition During Calyceal Development and Principal Cell Polarity.

Figure 22: Experimental design and tissue analysis for time series SBEM.

Figure 23: Clustering of cells and quantification of somatic inputs at P2.

Figure 24: Exuberant innervation of MNTB at P2.

Figure 25: Separation of cells and beginning of rapid nerve terminal growth at P3.

Figure 26: All cells contacted by large terminals at P4.

Figure 27: Continued growth and competition at P6.

Figure 28: Branches of parent axon do not converge onto the same MNTB cell.

Figure 29: Competition and population growth dynamics of calycigenic terminals.

Figure 30: Electrophysiology methods underestimate innervation.

Figure 31: Competing inputs innervate distinct domains on MNTB somatic surface.

Figure 32: Summary models for dynamics of calyx growth and competition.

Figure 33: Logistic Curve Fitting for Comparison of Polarity Curves.

Figure 34: Principal Cell Nuclei are Eccentric.

Figure 35: Quantification of Nuclear Eccentricity and Principal Cell Growth.

Figure 36: Nuclear Eccentricity and Principal Cell Growth by Developmental State at P3.

Figure 37: Calyceal Terminals are Polarized with Respect to the Nucleus.

Figure 38: Polarity Curves by Postnatal Day.

Figure 39: Polarity Curves by Developmental State (P3 Only). 
Figure 40: Competing Subtype K-Means Clustering Analysis.

Figure 41: Antipolar Calyces in Later Postnatal Development.

Figure 42: Surface Area Expansion and Somatic Spicules.

Figure 43: Average Total Surface Areas by Postnatal Day or Developmental State (P3).

Figure 44: Shift in Bisector Plane Defining Cytoplasmic and Nuclear Poles.

Figure 45: Glial Process Coverage of Principal Cells.

Figure 46: Percentage of Glial Coverage of Cell Surface with Spicules.

Figure 47: Dendrite Angles with Respect to Polarity Axis.

Figure 48: Axodendritic Association and Dendritic Pruning.

Figure 49: Comparison of Axodendritic Association for Competing and Dominant Developmental Categories.

Figure 50: MNTB of KASH/Engrailed-Cre Overexpression Mouse at P6.

Table 1. Image Volumes and Analysis 


\section{List of Abbreviations}

2P: two-photon

ABR: auditory brainstem response

ACSF: artificial cerebrospinal fluid

ALDH1L1: aldehyde dehydrogenase 1 family member L1

AMPA: $\alpha$-amino-3-hydroxy-5-methyl-4-isoxazolepropionic acid

AMPAR: $\alpha$-amino-3-hydroxy-5-methyl-4-isoxazolepropionic acid receptor

AP: action potential

AP5: (2R)-amino-5-phosphonovaleric acid

aPKC: atypical protein kinase $\mathrm{C}$

ASA: apposed surface area

Atoh: atonal basic helix-loop-helix transcription factor

ATP: adenosine triphosphate

AVCN: anteroventral cochlear nucleus

$A Z$ : active zone

BAl1: brain-specific angiogenesis inhibitor 1

BMP: bone morphogenetic protein

BMPR: bone morphogenetic protein receptor

BSE: back-scattered electron

CAST: cytomatrix at the active zone structural protein

CES: cochlear extramural stream

CF:PC: climbing fiber:Purkinje cell

$\mathrm{CN}$ : cochlear nucleus

CNTN: contactin

$\mathrm{CH}$ : calyx of Held

CP: cytoplasmic pole

Cre: Cre recombinase

CREB: cyclic adenosine monophosphate response element binding protein

CTZ: cyclothiozide

DAP: depolarizing action potential 
D-APV: (2R)-amino-5-phosphonovaleric acid

DCC: deleted in colorectal cancer protein

DCN: dorsal cochlear nucleus

DIC: differential interference contrast

dLGN: dorsolateral geniculate nucleus

Dmdo: Diminuendo mutant

dn/dn: deafness mouse

DRP1: dynamin-related protein 1

Dyn: dynamin

E\#: embryonic day \#

EdU: 5-ethynyl-2'-deoxyuridine

Egr2: early growth response 2 protein

EGTA: egtazic acid

ELKS: protein rich in the amino acids $E, L, K$, and $S$

EM: electron microscopy

En1: engrailed 1 homeobox protein

Eph: erythropoietin-producing human hepatocellular protein/receptor

EPSC: excitatory post-synaptic current

Exo: exocyst protein

Flp: Flp recombinase

FMR: Fragile $X$ mental retardation protein

FoxP1: forkhead box P1 transcription factor

Fz3: frizzled 3

GABA: gamma-Aminobutyric acid

gASA: glial apposed surface area

GBC: globular bushy cell

GFAP: glial fibrillary acidic protein

GFP: green fluorescent protein

GLAST: glutamate aspertate transporter

GLT-1: glutamate transporter 1 
GPI: glycophosphatidylinositol

GDP: guanosine diphosphate

GTP: guanosine triphosphate

Hox: homeobox-containing

11: input 1 (largest input)

12: input 2 (second largest input)

IHC: inner hair cell

ILD: interaural level difference

ITD: interaural time difference

$\mathrm{K}-\mathrm{W}$ : Kruskal-Wallis H-test

KASH: Klarsicht, ANC-1, Syne homology domain

KYN: kynurenate

LGL: lethal giant larva

LINC: linker of nucleoskeleton and cytoskeleton complex

LIS1: lissencephaly-related protein 1

LKB1: liver kinase B1

LNTB: lateral nucleus of the trapezoid body

LSO: lateral superior olive

MAC: mitochondria-associated adherens complex

MafB: V-maf musculoaponeurotic fibrosarcoma oncogene homolog B transcription factor

mGluR: metabotropic glutamate receptor

mIPSC: miniature inhibitory postsynaptic currents

miR: microRNA

MNTB: medial nucleus of the trapezoid body

mRNA: messenger RNA

MSO: medial superior olive

M-W: Mann-Whitney U-test

NB: neural recognition molecule

$\mathrm{N}-\mathrm{CP}$ : nucleus-cytoplasmic pole distance

NG2: neural/glial antigen 2 
NMDA: N-methyl-D-aspartate

NMJ: neuromuscular junction

NMDAR: N-methyl-D-aspartate receptor

N-NP: nucleus-nuclear pole distance

NP: nuclear pole

nSIC: neuronal slow inward current

OPC: oligodendrocyte precursor cell

P\#: postnatal day \#

Par: partitioning defective protein

PBS: phosphate buffered saline

PC: principal cell

PF: parallel fibers

PKC: protein kinase C

PMCA2: plasma membrane calcium ATPase pump

PSD: postsynaptic density

PV: parvalbumin

PVCN: posteroventral cochlear nucleus

r\#: rhombomere \#

rAAV: recombinant adeno-associated virus

Rac1: Rac family small GTPase 1

RalA: Ras-related protein A

RGC: retinal ganglion cell

Rim: Regulating synaptic membrane exocytosis protein

RMP: resting membrane potential

RNA: ribonucleic acid

Robo: roundabout receptor

ROSA: Gt(ROSA)26Sor promoter

RPTP: receptor protein tyrosine phosphatase

RRP: readily releasable pool

SAD: Synapses of the amphid defective kinase 
SBC: spherical bushy cell

SBEM: serial block-face scanning electron microscopy

Sec: secretory protein

SEM: scanning electron microscope

SMAD: combination of C. elegans "small" worm phenotype and Drosophila "Mothers Against Decapentaplegic"

SOC: superior olivary complex

SPON: superior paraolivary nucleus

SSEM: serial section electron microscopy

SSR: subsynaptic reticulum

sv: synaptic vesicle

SYT: synaptotagmin protein

TGF- $\beta$ : transforming growth factor $\beta$

Tiam1: T-cell Lymphoma Invasion and Metastasis 1

TrkB: tropomyosin receptor kinase B

TTX: tetrodotoxin

Vangl1/2: Van Gogh-like protein 1 and 2

VCN: ventral cochlear nucleus

VGCC: voltage-gated calcium channel

vGLUT: vesicular glutamate transporter

VNLL: ventral nucleus of the lateral lemniscus

Wnt: wingless-type protein 


\section{Chapter 1: Introduction and Literature Review}

\section{The Nervous System}

The nervous system is arguably the most important and complex system known to man. It consists of two major classes of cells: neurons and glia. Neurons are comprised of three major parts. Dendrites, from the Greek "dendron” for tree, are usually large, multi-branching processes extending from the cell body that receive signals from as many as thousands of other neurons. These signals, in the form of either a positive or negative electrical potential, are transmitted down the dendrite to the cell body, the location of the cell nucleus and major center of protein production. Multiple signals from one or more dendrites, as well as any signals transmitted directly to the cell body, are integrated across the cell body membrane. If this electrical potential exceeds a threshold (typically a depolarization from an average resting state of $-70 \mathrm{mV}$ to between -50 to $-55 \mathrm{mV}$ ), an all-or-nothing response — the action potential (AP) —is triggered in the initial segment of the third major structure called the axon. Each neuron typically has one axon, which connects the neuron to one (or many) other neurons through end processes called nerve terminals. The AP propagates down the axon to the terminals, where the electrical signal is transduced into a chemical signal at specialized structures called synapses; each nerve terminal can contain from one to hundreds of synapses. At the synapse, the AP triggers release of molecules known as neurotransmitters into the space between the transmitting-or presynaptic—cell and the receiving—or post-synaptic—cell. This space is known as the synaptic cleft. Multiple forms of neurotransmitter have been identified, and these neurotransmitters can interact with a wide range of receptors — either metabotropic or ionotropic —on the post-synaptic cell. Metabotropic receptors, such as G-protein coupled receptors, elicit a response in the postsynaptic cell upon ligand binding by intracellular activation of second messenger proteins. The response to metabotropic receptor activation can affect the whole cell and can last for an extended period. Ionotropic signaling, on the other hand, is localized to the immediate area of 
binding and is transient; neurotransmitter binding causes a conformational change in the ionotropic receptor that opens a channel permeable to specific ions and allows current to flow through the membrane. Only one of two responses can be elicited: excitatory (depolarizing), which brings the post-synaptic cell closer to firing, or inhibitory (hyperpolarizing), decreasing the likelihood of axon potential firing. Single neurons have therefore been classically categorized as either excitatory or inhibitory based on the response they elicit from their post-synaptic partner.

Unlike the singular signal transmission function of neurons, glial cells perform a wide array of functions in the nervous system. In the early developing brain, glial cells called radial glia provide both a source for new cells—both neurons and glia-and a scaffold of processes that guide cells to their appropriate locations. As the brain matures, four major types of glial cells are recognized: astrocytes, oligodendrocytes, microglia, and oligodendrocyte precursor cells (OPCs). Astrocytes, so named for their star-like morphology, wrap blood vessels to support the blood-brain barrier, envelop nerve terminals to both insulate them and help clear neurotransmitter, and provide structural support. Oligodendrocytes produce a substance called myelin, which wraps axons to both insulate them and allow for fast transmission of the AP. Microglia are the major immune cells of the brain, serving to both protect it from infection and clear away debris such as dead cells and overabundant nerve terminals. Finally, OPCs are the most recent glial subtype to be described. They provide a pool of cells that generate the oligodendrocyte population and can replace oligodendrocytes that have died, though they may have other functions that have yet to be described.

\section{Mouse Nervous System Development}

Although there are distinct differences in size, developmental timeline and specific brain structures, the overall development and structure of the vertebrate nervous system is largely conserved across species (Rice and Barone, 2000; Semple et al., 2013). In the mouse, the tissue that will eventually become the brain and spinal cord is formed in a process called 
neurulation, which begins around embryonic day (E)7 and lasts until approximately E11 (Rice and Barone, 2000). In this process, the ectoderm — the outer layer of the disc-shaped embryoundergoes a series of infoldings to form a cylindrical cord-shaped structure, the notochord, and an adjacent layer of tissue, the neural plate, contained within a superficial infold called the neural groove (Tam and Behringer, 1997; Desesso et al., 1999). The neural groove bends inward and fuses in at least 4 locations to form the neural tube at approximately E8 (Desesso et al., 1999; Jacobson and Tam, 1982). Around this same time, three distinct swellings of the anterior portion of the neural tube can be observed: the prosencephalon, mesencephalon, and rhombencephalon; these three areas form the basis for the developing brain. The remainder of the neural tube forms the spinal cord and peripheral nervous system, the discussion of which is beyond the scope of this dissertation (Fig.1; Jacobson and Tam, 1982; Desesso et al., 1999; Rice and Barone, 2000; Ishikawa et al., 2012) The prosencephalon and rhombencephalon further subdivide into the telencephalon, diencephalon, and metencephalon and myelencephalon, respectively; the mesencephalon, or midbrain, remains structurally undivided at this stage (Desesso et al., 1999; Jacobson and Tam, 1982; Rice and Barone, 2000). The telencephalon develops into the cerebral cortex, hippocampus, and limbic and olfactory systems, the diencephalon into the optic system, thalamus and hypothalamus, and the mesencephalon forms the tectum and tegmentum of the midbrain (Rice and Barone, 2000).

The auditory structures that are the basis of this dissertation are located within the mouse hindbrain. This brain region can be subdivided into seven physically distinct, but transient, subregions known as rhombomeres ( $r$ ). Prior to a swelling of the rhombomeres to allow for physical distinction, these sub-regions can be identified through differential protein expression; in particular, four clusters of homeobox-containing (Hox-1,Hox-2,Hox-3, and Hox-5) genes are expressed in a rostral-to-caudal pattern corresponding to their 5' to 3' arrangement on the chromosome (Graham et al., 1989; Hunt et al., 1991; Nieto et al., 1992; Irving et al., 1996) (Fig. 
2A-B). Rhombomeres represent divisions between regions of lineage-restricted cells, with little to no migration of neurons occurring across rhombomeric boundaries (Jimenez-Guri et al., 2010). The dorsal region of the rhombomeres does not remain closed; at approximately E9, the previously closed edges of the neural tube expand outward and form the rhombic lip, an important region of proliferating neuroepithelium that gives rise to multiple cell types in the cerebellum, medulla and pons (Wingate, 2001; Hunter and Dymecki, 2007) (Fig. 2C).

\section{Mouse Auditory Brainstem}

\section{Anatomy}

The mouse auditory brainstem is the initial site of entry for auditory information in the central nervous system and is vital to the integration of binaural sound cues for localization of sounds in three-dimensional space (Cant and Benson, 2003; Davis, 2005). Broadly, the auditory brainstem consists of the cochlear nucleus $(\mathrm{CN})$ and the superior olivary complex (SOC), both of which consist of smaller subdivisions of cell groups, and the ventral nucleus of the lateral lemniscus (VNLL) (Fig. 3A). Auditory information is conveyed from the hair cells of the cochlea through spiral ganglion neurons-bipolar neurons that form the vestibulocochlear (VIII) nerve. Passing into the CNS through the internal auditory meatus of the skull, this nerve enters the CN. The $\mathrm{CN}$ can be divided into two major subdivisions: the ventral cochlear nucleus (VCN) and the dorsal cochlear nucleus (DCN). The VCN is further subdivided by the VIII nerve, which bifurcates upon entering the VCN and demarcates the anatomical divide between the anterior (AVCN) and posterior (PVCN) cochlear nuclei (Webster and Trune, 1982; von Gersdorff and Borst, 2002). In addition to their spatial distinction, the VCN and DCN also differ in cellular organization; the DCN has a layered, cerebellar-like structure, while the VCN possesses no clear well-defined ordering of cells (Webster and Trune, 1982; Hackney et al., 1990). These two areas are similar in one respect, however: tonotopic organization. The innervation patterns of the AVCN, PVCN and DCN mirror the frequency progression of the cochlea, with lower 
frequency representations in the anteroventral areas of the nuclei progressing to higher frequencies in the posterodorsal regions (Muniak et al., 2013; Muniak and Ryugo, 2014; Spirou et al., 1993; Fig. 3B). This tonotopic organization is recapitulated in multiple nuclei throughout the auditory circuit (Kandler et al., 2009). Although both the PVCN and DCN contribute to multiple types of acoustic processing, a detailed description of these areas is beyond the scope of this dissertation. We shall instead turn our focus in more detail to the AVCN.

The AVCN consists of 6 major types of neurons: large and small spherical bushy cells (SBCs), globular bushy cells (GBCs), multipolar cells, small cells, and granule cells (Osen, 1969;

Webster and Trune, 1982; Hackney et al., 1990; Doucet and Ryugo, 2006). The anterior pole of AVCN contains mostly large SBCs, mixing gradually into the smaller SBCs found caudally. In contrast, the transition between small SBCs and the most caudal cell type, the GBCs, is more stark and pronounced (Webster and Trune, 1982). SBCs and GBCs also differ in the location of their nuclei; SBCs tend to have a more centered nucleus, while GBC nuclei are eccentrically located (Osen, 1969; Webster and Trune, 1982; Hackney et al., 1990). Interspersed with the spherical and globular bushy cells are multipolar cells, classified into two major groups: onset neurons, which are large stellate cells that fire at the onset of a sound, and chopper cells, a smaller class of stellate cell that respond to a stimulus with a train of regularly spaced APs (Smith and Rhode, 1989; Arnott et al., 2004; Oertel et al., 2011). Collectively, the SBCs, GBCs, and multipolar cells comprise the magnocellular core of the AVCN. Directly adjacent to these cells is a layer of small cells, termed the "small cell cap" (Osen, 1969), that receive both ascending (from the cochlea) and descending (towards the cochlea) auditory input and may participate in a feedback circuit to the inner ear (Ye et al., 2000; Ryugo, 2008). Similarly, granule cells form a continuous lamina (the granule cell layer) covering the $\mathrm{VCN}$ and extending into the DCN, and these cells receive both ascending and descending auditory input, as well as multimodal input from outside of the auditory system (e.g., vestibular nuclei, cuneate nucleus for 
touch and proprioception, Roller's nucleus for control of eye gaze) (Ryugo, Haenggeli, and Doucet, 2003). The granule cell layer also contains an array of other cell types: unipolar brush cells, Golgi cells, and chestnut cells, all of which may provide interesting auditory processing functions but are beyond the scope of this dissertation (Mugnaini et al., 1980; Weedman and Ryugo, 1996). A summary of the AVCN structure and cellular relationships can be seen in Fig. 3C (modified from Webster and Trune, 1982).

Axons from most of the cells in the AVCN leave the nucleus together in an axon tract known as either the ventral acoustic stria or the trapezoid body. For simplicity, we will focus on axonal projections of the three major cell types of the AVCN: large and small SBCs, and GBCs. These axons innervate the cells of the SOC and VNLL to different extents on both the ipsilateral and contralateral sides of the brain (Fig. 4A; Stotler, 1953; Warr, 1966, 1972; Brownell, 1975; Cant and Benson, 2003). The SOC, which plays a vital role in azimuthal localization of sound as initially proposed by Jeffress (Jeffress, 1948), includes four primary nuclei-the medial (MSO) and lateral (LSO) superior olive, superior paraolivary nucleus (SPON), medial nucleus of the trapezoid body (MNTB)—as well as multiple periolivary nuclei (Fig.4B; Ramón y Cajal, 1909; Paxinos et al., 2012). Although a complete study of the process of binaural sound localization is beyond the scope of this dissertation, a brief understanding will be useful for understanding the circuit dynamics underlying this research.

\section{Binaural Sound Localization}

Two methods are utilized by the auditory brainstem to compute the location of a sound in horizontal space (i.e., left or right of the midline of sight) as described separately by Thompson and Rayleigh (Thompson, 1882; Rayleigh, 1907). Interaural level difference (ILD) is the difference in perceived intensity or amplitude of a sound between the two ears due to attenuation by the head. Rayleigh showed that this difference in intensity $(\Delta \mathrm{l})$ can be expressed as follows: 


$$
\Delta I=\frac{3}{4}\left(\frac{2 \pi r}{\lambda}\right)^{4}
$$

where $2 \pi r$ is the circumference of the head and $\lambda$ is the wavelength of the sound (Rayleigh, 1907). As the wavelength increases, or the size of the head decreases, the difference in intensity perceived by each ear becomes vanishingly small. Thus, ILD is useful for localizing high frequency (low wavelength) sounds but is less useful at low frequency (high wavelength). A second method, interaural time difference (ITD), is used to discriminate in these cases. The physical distance between the two ears causes a delay in detection at the further ear. This amounts to a phase shift in the sound wave that, when compared to the originally detected wave, provides information on location in the horizontal plane (Rayleigh, 1907). These two methods of computation are not exclusive by wavelength in the mammalian auditory system, however; ILDs can be significant at low frequency if the source is sufficiently close to the head (Brungart and Rabinowitz, 1999), and ITDs at high frequency can be discriminated even in the presence of a low frequency carrier (Henning, 1974).

Each of the major nuclei of the SOC plays a different but complementary role in binaural sound localization through computation of either ITD or ILD. Early studies in the cat were instrumental in defining the binaural circuitry necessary to accomplish these tasks (Stotler, 1953; Warr, 1966; Lindsey, 1975; Caird and Klinke, 1983). The MSO, which receives excitatory input from both ipsi- and contralateral spherical bushy cells of the AVCN, is essential for computation of ITDs (Masterton et al., 1967). As described by Grothe et al., this is an extremely temporally precise process, requiring neurons to "resolve differences in the time of arrival of the sound at each ear that are almost two orders of magnitude shorter than the duration of action potentials bearing that information" (Grothe et al., 2010). To accomplish this, Jeffress proposed the idea of "delay lines"-converging binaural pathways that can detect coincidence between the signals from each ear and, based on the cells activated, define the location of origin within horizontal space 
(Fig. 5A; Jeffress, 1948). As the MSO integrates excitatory input from both AVCN, this is a logical location for such "delay lines" to converge, and this has been the generally accepted model for ITD since its inception. However, it fails to account for the inhibitory innervation to MSO neurons by both ipsilateral lateral nucleus of the trapezoid body (LNTB) and MNTB cells, themselves innervated by ipsilateral and contralateral GBCs, respectively (Spirou et al., 1990; Smith et al., 1991). The findings of Brand et al. and Pecka et al. in the gerbil have shown these glycinergic inputs to be indispensable for maintaining the precise sensitivity to ITD in the MSO necessary for sound localization; both showed that application of strychnine to abolish inhibitory input to MSO neurons caused a shift of response in these cells rendering them unable to detect ITDs. Interestingly, application of glycine —-the inhibitory neurotransmitter-had much the same effect, suggesting that not only inhibition but the timing of the inhibitory signal is necessary for proper ITD tuning (Brand et al., 2002; Pecka et al., 2008). As the SBCs that directly excite MSO neurons and the GBCs that indirectly inhibit them both exhibit "phase locking" (firing at a fixed frequency dictated by a periodic input, in this case sound frequency), a property necessary for conveying temporal information with high fidelity, this circuit is well constructed for computation of precise ITDs (Goldberg and Brown, 1969; Smith et al., 1991, 1993; Joris et al., 1994).

A second, complementary circuit for binaural sound localization in the auditory brainstem is involved in computation of ILD and involves neurons of the LSO. Cells of the LSO receive excitatory input from ipsilateral SBCs, as demonstrated through both axon degeneration assays with lesions in the AVCN and direct electrophysiological studies (Galambos et al., 1959; Warr, 1966; Caird and Klinke, 1983). Counterbalancing this, inhibitory inputs from the ipsilateral MNTB (Warr, 1972) attenuate firing of LSO neurons, with the amount of attenuation dependent upon how close in intensity the contralateral tone is to the ipsilateral tone (Galambos et al., 1959; Boudreau and Tsuchitani, 1968; Caird and Klinke, 1983). Similarly to the MSO, both strychnine and excess glycine blocked the binaural response in LSO neurons, suggesting a glycinergic 
mechanism for inhibition and a need for precise timed release for proper binaural circuit function (Moore and Caspary, 1983). A summary of the circuitry involved in computation of ITD and ILD can be found in Fig. 5B (Grothe et al., 2010).

Both systems share a common source of inhibition that ensures temporal precision: MNTB principal cells (PCs). These cells, a primary target of innervation from globular bushy cells in the contralateral AVCN, act as high-fidelity signal inverters; the glutamatergic input of the GBC is transformed into a glycinergic output with near sub-millisecond accuracy by the large, multisynaptic terminal formed between the GBC axon and the PCs of the MNTB, the calyx of Held (CH) (Fig. 5C; Ramón y Cajal, 1909; Schneggenburger and Forsythe, 2006). This terminal, named for the German anatomist Hans Held who first described it in his 1893 treatise "Die centrale Gehörleitung", occupies approximately $40-60 \%$ of the surface of the PC in the adult animal, making it one of the largest terminals in the mammalian central nervous system (Held, 1893). The number of active zones, a measure of terminal size and strength, can exceed 400 in the mouse and 2500 in the cat (Ryugo and Spirou, 2010; Baydyuk et al., 2016). Additionally, the large size of the calyx allows for direct patch-clamping of both it and its postsynaptic partner, making it an attractive target for electrophysiological research (Fig. 5D; Forsythe, 1994; Borst et al., 1995; Wimmer et al., 2004). Despite its large size, the development of the calyx of Held occurs over a short time period (3-5 days; Kandler and Friauf, 1993; Hoffpauir et al., 2006; Rodríguez-Contreras et al., 2008). This combination of size, accessibility, and short developmental period appear to make the MNTB:CH connection appealing as a model system for studying neural development. However, there are still outstanding questions regarding the development of this connection. As detailed in a later section, these questions will form the basis for the experimental work detailed herein. 


\section{Development of the MNTB:CH Connection}

\section{Cellular Development}

Both the AVCN and MNTB arise from the rhombomeres of the mouse hindbrain. As early as embryonic day $(E) 11$, neurons that give rise to the AVCN can be identified in the caudal portion of the lower rhombic lip in the mouse (Pierce, 1967; Martin and Rickets, 1981). These cells reside in rhombomeres 2-3, as determined by sparse expression of Wingless-Type 1 (Wnt-1) compared to surrounding neurons (Nichols and Bruce, 2006); the origin of these cells was further confirmed through intersectional fate mapping of the lower rhombic lip using a Cre/Flp system to track both rostrocaudal and dorsoventral position of cells from early embryonic stages $(\mathrm{E} 11.5,14.5,16.5)$ to adult (Fig. 6A; Farago et al., 2006). Proper expression of the Hox genes is necessary for lineage restriction of AVCN neurons to r2-3, with Hoxb1-null mice demonstrating ectopic expression of Atoh7 in the PVCN and-conversely_ animals lacking Hoxa2 showing Atoh7+ cell depletion in the AVCN (Fig. 6B). Atoh7 (atonal basic helix-loop-helix transcription factor, also known as Math5) is an AVCN-specific glutamatergic neuronal marker, and in adult mice is found exclusively in globular and spherical bushy cells (Di Bonito et al., 2013). A second member of this protein family, Atoh1 (Math1), is also necessary for VCN development, and has been utilized to examine the migration of VCN cells. Transgenic mice expressing the LacZ reporter along with Atoh1 from E11.5 to E18.5 showed a distinct migratory stream of cells, the cochlear extramural stream (CES), migrating tangentially from the caudal rhombic lip along the ventrolateral surface of the developing dorsal cochlear nucleus and populating the area of the VCN found in adults (Fig. 6C; Wang et al., 2005).

Similar experiments to those defining the origins of the AVCN have also been carried out to elucidate the birth location and migration of principal neurons in the MNTB. Autoradiographic evidence suggests MNTB cells are born between E11-12 (Pierce, 1973). Xgal staining in Egr2 $^{\text {Cre }} ; R O S A^{R 26 R}$ mice at postnatal day $(\mathrm{P}) 30$ suggests the majority of MNTB cells originate 
from rhombomeres 3 and 5 , though its position relative to these rhombomeres argues for a strictly r5 lineage; Egr2, also known as Krox20, is a transcription regulatory factor expressed selectively in these rhombomeres (Fig.7A; Maricich et al., 2009). Similar experiments using Hoxb1 ${ }^{\text {Cre }} ;$ ROSA $^{\text {R26R }}$ animals only stained a portion of the MNTB, but suggest that rhombomere 4 may also contribute to this nucleus (Fig. 7B; Maricich et al., 2009; Marrs et al., 2013). In order to more specifically determine the embryonic origins of the SOC nuclei, Marrs et al. characterized a set of markers for differentiating between cell types of the SOC during development: En1 (engrailed 1, homeobox protein), expressed in the MNTB, VNTB, LNTB, and the lateral olivocochlear shell neurons surrounding the LSO; MafB (V-maf musculoaponeurotic fibrosarcoma oncogene homolog B, transcription factor) expressed in the LSO and MSO; and FoxP1 (forkhead box P1, transcription factor), expressed in SPON, MNTB and LNTB. By applying these markers to embryonic ages, Marrs et al. demonstrated that both distinct expression patterns and cell positions that distinguish most nuclei of the SOC can be identified as early as E14.5. The exception to this were the nuclei of the trapezoid body, which appeared clustered at E14.5 and only showed mediolateral migration at E16.5 and later. By E17.5, the presumptive MNTB could be identified as a medial cluster of En1 and FoxP1-positive cells in approximately the same location as adult MNTB (Fig. 7C; Marrs et al., 2013).

\section{VCN Axon Pathfinding}

Axonal outgrowth leading to proper wiring between GBCs of the AVCN and PCs of the MNTB occurs concurrently with embryonic development and migration of acoustic neurons. A detailed description of VCN axon extension was accomplished by Howell et al. through use of Dil and DiD injections in the cochlear nucleus beginning at E12.5 (Howell et al., 2007). At E13, axons begin to extend from the cochlear nucleus, and as early as E13.5 these axons have extended to the ventral surface of the brain near the midline, with a small number of axons decussating by E14.5 (Fig. 8A; Kandler and Friauf, 1993; Howell et al., 2007). Proper specification of cell fate 
by Hox genes appears to be a key initial factor in appropriately establishing these axons. Removal of Hoxb1 or Hoxb2 causes aberrant PVCN connection with the contralateral MNTB as assessed at P8 in the mouse (post-calyx development), again suggesting a cell fate switch from $\mathrm{PVCN}$ to $\mathrm{AVCN}$ in this cell population. Interestingly, when $\mathrm{Hoxa2}^{\text {flox }}$ is inactivated using Wnt1::Cre recombination, AVCN cells that typically innervate the contralateral MNTB are found to instead innervate the ipsilateral MNTB (Di Bonito et al., 2013). Di Bonito et al. note that this finding is strikingly similar to conditional knockout results from Krox20::cre, Robo3 $3^{10 x / 10 x}$ mutant mice, and show that, in fact, Robo3 expression is at least partially regulated by Hoxa2 (Renier et al., 2010; Michalski et al., 2013). Robo (roundabout receptor) proteins, initially identified in Drosophilia, have traditionally been associated with repulsion of axons attempting to cross the midline through binding with Slit proteins (Evans and Bashaw, 2010; Nawabi and Castellani, 2011). The Slit-Robo system, along with other protein-protein interactions such as DCC (deleted in colorectal cancer)-netrins and Ephs-ephrins (erythropoietin-producing human hepatocellular protein/receptor), are integral to axon guidance and are highly conserved across species (Evans and Bashaw, 2010). In addition to Robo3, Howell et al. showed that both Robo1 and Robo2 RNA is expressed in the cochlear nucleus as early as E13.5 through hybridization with robo-1 and robo-2 antisense RNA probes; RNA expression of Robo1/2 overlaps at least partially with the site of expression of DCC, suggesting cells in the cochlear nucleus may express all three proteins (Howell et al., 2007). Complementary to this, Howell et al. also found that slit1 (but not slit 2/3) and netrin-1 are expressed at the midline in the developing auditory brainstem at E13.5 (Fig. 8B, Howell et al., 2007). Although a later study by Renier et al. showed the presence of slit2/3 as well as slit1 at the midline, in-situ hybridization for this RNA was done at E11, possibly demonstrating a shift in slit expression at the midline over this time period (Renier et al., 2010). While Slit-Robo binding is repulsive, DCC-netrin binding is attractive to axonal outgrowth. DCCnull mice show no outgrowth of axons from the $\mathrm{VCN}$ until at least $\mathrm{P} 0$, and these axons only reach the ipsilateral SOC and never approach the midline (Howell et al., 2007). Slit-Robo 
binding also abrogates the effect of DCC-netrin on axon growth, with the important exception of Robo3; Robo3 in mammals acts as an atypical Robo protein, indirectly increasing midline attraction (Zelina et al., 2014; Friocourt and Chédotal, 2017). Robo3 binds DCC in a receptor complex but does not directly bind netrin-1. Instead, in the presence of netrin-1—most likely through its binding of DCC—Robo3 is phosphorylated through Src kinases, possibly c-Src (Zelina et al., 2014). Although the intracellular consequences of this association have not yet been clarified, Zelina et al. show that loss of Robo3 in precerebellar neurons of the pontine nucleus inhibited the ability of these cells to migrate towards the midline, even though DCC was expressed at the cell surface in similar quantity to wild-type mice (Zelina et al., 2014). The attractive behavior of Robo3 is complementary to the antagonistic effect that this protein appears to exert on Slit repulsion through Robo1/2, an effect possibly accomplished through Robo3-induced degradation of Robo1/2 as demonstrated in vitro (Sabatier et al., 2004; Jaworski et al., 2010; Li et al., 2014). Additionally, two Robo3 isoforms are expressed in spinal commissural neurons, with Robo3.1 acting prior to midline crossing to affect midline attraction, and Robo3.2 present in post-crossing fibers to increase midline repulsion; which of these two Robo3 isoforms are present in GBC crossing fibers has yet to be determined (Chen et al., 2008). This sequence-axonal attraction by netrin and suppression of slit signaling, axons entering the midline, and a functional switch from attraction to repulsion to "push" axons into the contralateral hemisphere-is a common theme throughout the developing nervous system (Nawabi and Castellani, 2011).

While traversing the developing hindbrain, GBC axons course through the ipsilateral MNTB on their way to the midline and-eventually - the contralateral MNTB. However, in wild-type mice the calyx of Held develops strictly contralaterally, suggesting that molecular guidance cues may exist to either repel VCN axons from the ipsilateral MNTB or attract them towards the contralateral MNTB or both. As mentioned previously, Robo3-deficient mice have aberrant 
innervation of the ipsilateral MNTB by VCN axons. These misplaced calyces do not develop properly, maintaining immature firing properties into adulthood, and PCs in these cases also possess multiple presynaptic partners (Renier et al., 2010; Michalski et al., 2013). No electrophysiological deficiencies were found when Robo3 was removed after midline crossing, however, suggesting that crossing the midline-and not Robo3 itself-affects the targeting and maturation of the calyx of Held (Fig. 9A, Michalski et al., 2013). The repulsion of VCN axons from the ipsilateral MNTB has been shown to be mediated in part by Eph-ephrin binding. Eph proteins represent the largest family of receptor tyrosine kinases, and their signaling can be bidirectional, with Eph "forward" signaling and ephrin "reverse" signaling causing changes in behavior in their respective cells. Ephs are subdivided into $A$ and $B$ types based on protein homology and affinity for ephrin binding; ephrins are similarly divided into two categories, with ephrinA proteins tethered to the cell membrane through a glycosyl phosphatidylinositol—or GPI-anchor and ephrinB being a transmembrane protein. Although these proteins generally bind A-to-A or B-to-B, there are exceptions, most notably ephrin-A5, which can bind to EphB2 in addition to EphA receptors (Klein, 2004). Null mutations in either ephrin-A2 or ephrin-A5 have been shown to induce abnormalities in the auditory brainstem response (ABR) in mice (Yates et al., 2014). In wild-type animals, ephrin-A2 is expressed in VCN from E17-P12, with expression decreasing from P4-P12. Ephrin-A2 is also expressed in areas surrounding MNTB PCs, with expression most prominent at P4. In contrast, ephrin-A5 is expressed in PCs as early as E17, with expression more well-defined at P4 and decreased by P12. It is present only diffusely in VCN, but with the same temporal pattern of expression. Ephrin-A2 KO, ephrin-A5 KO, and ephrin-A2/A5 DKO mice all exhibit growth of both contralateral and ipsilateral calyces, suggesting one function of these proteins is to prevent ipsilateral calyx formation (Abdul-latif et al., 2015). EphB2, the binding partner for ephrin-A5 as well as ephrin-B2, is expressed during development in VCN, and both EphB2-null and ephrin-B2-null mice show similar ipsilateral calyx growth to the ephrin-A2 or ephrin-A5 knockouts; although more study is necessary to reveal the 
exact molecular pathway, these results may indicate a common pathway for Eph-ephrin signaling in assuring strictly contralateral calyx formation (Fig. 9B). Importantly, when the intracellular portion of EphB2 is replaced with $\beta$-galactosidase, ipsilateral calyx growth is still inhibited. Thus, it is the "reverse" signaling of the ephrin binding partner, and not the "forward" signal from EphB2, that is essential for prevention of this erroneous connection (Hsieh et al., 2010).

\section{Calyx of Held Morphological Development}

Functional connection between the VCN and MNTB is present as early as E17 in mice, where stimulation in VCN can evoke APs in principal cells (Hoffpauir et al., 2010). As mentioned previously, this connection is accomplished in the adult through the large axosomatic terminal, the calyx of Held. Three stages of the developing $\mathrm{CH}$ were described by D. Kent Morest in 1968 using Golgi impregnation of multiple developmental ages in cats, rats, opossums, and rabbits: the migratory growth cone, the protocalyx, and the young calyx (Morest, 1968b). Migratory growth cones that have the potential to form the calyx stem from axons that originate in the ventral acoustic stria ranging in size from $0.5 \mu \mathrm{m}$ to $2 \mu \mathrm{m}$ in diameter (Kandler and Friauf, 1993). These growth cones typically end in either filipodial- or lamellipodial-like structures with multiple thin processes projecting outward (Fig. 10A, Morest, 1968a; Kandler and Friauf, 1993). Many of the initial contacts of these axons with PCs take place on dendrites (Hoffpauir et al., 2006, 2010; Rodríguez-Contreras et al., 2008). Small, flattened, spoon-shaped contacts between VCN axons and the PC soma-representing the initial protocalyx — can first be identified at P2 (Hoffpauir et al., 2006; Rodríguez-Contreras et al., 2008). Protocalyces often have long radiating processes that may contact neighboring PCs (Kandler and Friauf, 1993; RodríguezContreras et al., 2008; Hoffpauir et al., 2010). Protocalyceal terminals grow rapidly from P2-P4, with the majority of cells possessing a single large terminal (protocalyx or young calyx) by P4

(Fig. 10B, Kandler and Friauf, 1993; Hoffpauir et al., 2006, 2010; Rodríguez-Contreras et al., 
2008; Soria Van Hoeve and Borst, 2010; Xiao et al., 2013). The protocalyx maintains its solid, single compartment structure until approximately the onset of hearing (P10-12, Ehret, 1976, 2005; Hammerschmidt et al., 2012) when the terminal is re-sculpted into its mature shape; the solid protocalyx restructures into multiple stalks and swellings connected by thin $(\sim 1 \mu \mathrm{m}$ diameter) necks and spread across the surface of the PC in a process called fenestration (Fig. 10C, Kil et al., 1995; Rowland et al., 2000; Wimmer et al., 2006; Ford et al., 2009). This maturation process is tonotopically graded, with calyces in the medial, high-frequency portion of the MNTB fenestrating before the lateral, low-frequency sensitive areas (Ford et al., 2009).

\section{Protein Pathways Involved in Calyx Growth}

Substantial changes in gene expression occur during the development of the calyx, with over 500 genes differentially expressed during the postnatal developmental period from P0-P6 (Kolson et al., 2016). Although most of these genes have not been investigated regarding calyx growth and maturation, several key proteins have been identified in this process. These include bone morphogenetic proteins (BMP), Synapses of the Amphid Defective (SAD) kinases, Regulating Synaptic Membrane Exocytosis proteins (Rim1/2), contactin 5 (CNTN5 or NB-2), dynamins, Exo70, and miR-96.

BMPs are secreted molecules in the transforming growth factor $\beta$ (TGF- $\beta$ ) family that act as ligands for type-I and type-II receptors and lead to gene expression changes in their target cell through recruitment and phosphorylation of SMAD proteins (Katagiri and Watabe, 2016). Both BMPs and their receptors are highly expressed in the MNTB at P3, when substantial protocalyceal growth occurs (Xiao et al., 2013). Conditional knockout of floxed BMP receptor (BMPR) type 1a using Krox20-Cre crossed with a global knockout of BMPR1b led to smaller protocalyces, poly-innervation of PCs as late as P16, and reduced excitatory post-synaptic current (EPSC) amplitudes (Fig. 11A). As phosphorylated SMAD proteins are present in the nuclei of both VCN and MNTB cells at P2 and P9 (pSMAD1,5, and 8) and SMAD4 is present in 
the calyx at P3 and P9, activation of these proteins may be disrupted in the BMP knockout and cause the observed phenotype; unfortunately, immunohistochemistry for SMADs was only performed in wild type animals, so no information on location or activation of these proteins in BMP knockout is available (Xiao et al., 2013). Although application of the SMAD1/5/8 phosphorylation inhibitor LDN-193189 to an in vitro slice culture of MNTB resulted in smaller terminals, atypical growth characteristics of the slice culture make the results questionable (Kronander et al., 2017).

SAD kinases-SAD-A and SAD-B, both homologs of the C.elegans Par-1 polarity protein-are downstream targets of the liver kinase B1 (LKB1, mammalian homolog of C.elegans Par-4) and are essential in establishing neuronal polarity through axon initiation, growth, and elaboration (Shelly and Poo, 2011). Both SAD kinases are highly expressed in the developing calyx of Held and colocalize with vesicular glutamate transporter 1 and 2 (vGLUT1/2), membrane-bound proteins that transport glutamate into vesicles, and synaptotagmin-2 (SYT2), a $\mathrm{Ca}^{2+}$ sensor. When floxed SAD kinases are conditionally deleted from the MNTB using Cre under the postnatally expressed parvalbumin driver (PV-Cre), calyx growth is disrupted; in P24 PVCre/SAD ${ }^{K O}$ animals, calyces occupy $\sim 33 \%$ less of the PC surface than controls (Fig. 11B). Lilley et al. put forward two potential explanations for this. First, SAD kinases have been shown to phosphorylate tau and $\mathrm{y}$-tubulin, suggesting they may play a role in microtubule growth and stabilization necessary for the continued expansion of the calyx. Second, the SAD-B kinase phosphorylates RIM scaffolding proteins that have been implicated in the maintenance of calcium channel density and the readily releasable pool (RRP) of vesicles (Mittelstaedt et al., 2010; Han et al., 2011, 2015; Lilley et al., 2014). The RRP consists of vesicles that are positioned such that they can be more quickly released than others, and the size of the RRP and probability of release determine the amount of neurotransmitter released upon terminal activation (Kaeser and Regehr, 2017). In the developing calyx of Held (P5), postsynaptic 
densities (PSDs) are larger, with more docked vesicles than in the more mature calyx (P14); reduction in both PSD size and docked vesicles refines the release properties of the calyx, reducing the amount of glutamate released per AP, allowing for high frequency signal transmission by preventing the saturation of postsynaptic AMPA ( $\alpha$-amino-3-hydroxy-5-methyl4-isoxazolepropionic acid) receptors (Taschenberger et al., 2002). Adult active zones (AZs) in the calyx of Held are characterized by highly clustered calcium channels surrounded by a "donut" of vesicles connected by filaments, with one or several mitochondria located in or near the "donut hole", and a specialized network of cytoskeletal structures connecting the mitochondria to the $A Z$. Both the amount of channels per cluster and the size of the clusters increase from P7-P14 (Nakamura et al., 2015). The aggregation of presynaptic structures, termed the mitochondria-associated adherens complex (MAC), has been identified in the large endbulbs of Held innervating bushy cells of the VCN, terminals in the LNTB and the calyx of Held (Fig. 11C, Cant and Morest, 1979; Spirou et al., 1998; Rowland et al., 2000; Wimmer et al., 2006; Perkins et al., 2010; Nakamura et al., 2015). MACs are not apparent until P3 and, interestingly, the donut-like structure of synaptic vesicles does not form until P12, coinciding closely with sensitivity to airborne sound (Hoffpauir et al., 2006; Wimmer et al., 2006). The MAC-adjacent calcium channel clusters in the AZ also colocalize closely with RIM proteins, and conditional double knockout of RIMs show a dramatic reduction in docked vesicles at the AZ (Fig. 11D, Han et al., 2011; Nakamura et al., 2015). Additionally, RIM proteins have been shown to bind directly to both calcium channels and SYT2, and in the presence of $\mathrm{Ca}^{2+}$ this binding is enhanced (Coppola et al., 2001). When a mutated form of SYT2 is overexpressed in the calyx which disrupts the binding site of RIM proteins, vesicle release probability is lowered, and a reduction is seen in the RRP. RIM proteins also bind the CAST (cytomatrix at the active zone structural protein) and ELKS (protein rich in the amino acids $E, L, K$, and S) family of proteins, which have been implicated in the clustering of calcium channels in the calyx (Dong et al., 2018). Together, these findings suggest that SAD kinase and RIM proteins-along with their 
associated binding partners - may be key proteins in both the morphological and intra-terminal restructuring of the calyx of Held concomitant with changing patterns of activity associated with sensitivity to airborne sound. Further research is needed to directly link this signaling pathway to a specific stage in calyx development, however.

Contactins (CNTNs), as the name suggests, are glycophosphatidylinositol (GPI)-linked neural recognition and cell adhesion proteins of the immunoglobulin superfamily (Zuko et al., 2013). Six contactin homologs (CNTN1-6) have been identified with 40-60\% shared sequence homology between them (Bouyain and Watkins, 2010). Contactins bind multiple downstream partners, the most prominent being the receptor protein tyrosine phosphatases (RPTPs), a family of transmembrane proteins involved in cell-cell interaction that have been implicated in axon elongation, targeting and both pre- and post-synaptic organization (Dunah et al., 2005; Takahashi and Craig, 2013; Zuko et al., 2013). CNTN5 (NB-2) is expressed in both the VCN and calyces of Held in a developmentally specific manner (P2-P10), and global knockout of CNTN5 results in $8 \%$ of MNTB PCs lacking calyces and apoptosis of these cells by P15 (Fig. 11E, Toyoshima et al., 2009a, 2009b). Phenotypically, this leads to inter-peak latency increases in ABR between waves II-III (IIX nerve transmission to CN) and III-IV (CN-SOC transmission), which may lead to dysfunction in sound localization (Toyoshima et al., 2009b; ClaesdotterHybbinette et al., 2016). Cntn4 and Cntnap1 (contactin-associated protein 1) RNA have also been shown to be expressed in a developmentally specific manner in the MNTB, though the localization of the protein products and their function in calyx growth and maturation have yet to be investigated (Kolson et al., 2016).

The large guanosine triphosphatase (GTPase), dynamin, also plays a role in development of the calyx of Held. Dynamins—of which there are three identified subtypes (Dyn-1/2/3)—associate with lipid membranes and facilitate shape changes involved in vesicle scission, mitochondrial fusion and fission, and cytokinesis (Kar et al., 2017). The expression of dynamins increases in 
the MNTB postnatally, with Dyn-1 located in both the growing calyx and the MNTB PCs while Dyn-3 is located almost exclusively in PCs. Single conditional knockout of either floxed Dyn-1 or Dyn-3 using Krox20-Cre causes no noticeable change in calyx growth. However double knockout of these proteins causes a significant decrease in MNTB size and calyx area, as well as neuron and astrocyte loss and an increase in activated microglia. These changes only became apparent after P3, when protocalyces undergo rapid growth (Fig. 11F, Hoffpauir et al., 2006; Soria Van Hoeve and Borst, 2010; Fan et al., 2016). Multiple small, vGLUT1 positive puncta persist until P8 or later in these animals, but whether these represent unique sites of innervation—like the BMP1a/b-KO—or a monoinnervated state depleted of synaptic vesicles is unknown. Dyn-1/3 double knockouts also maintain immature electrophysiological properties both pre- and post-synaptically: lower frequency firing, slower rise and decay times, lack of switching from tonic (sustained bursting of APs with depolarization) to phasic (one to several APs fired with depolarization) firing, and higher resting membrane potentials compared to wild type animals (Fan et al., 2016). It is unclear, however, whether these electrophysiological defects precede the developmental dysfunction in calyx growth or are a result of it. Whether the dynamin-mediated disruption in $\mathrm{CH}$ :MNTB connectivity is directly related to lack of proper endocytosis or indirectly related to the loss of internalization of trophic factors—or both-also remains to be elucidated. There is evidence for GTP- and dynamin-independent endocytosis at the calyx of held in P7-10 rat brainstem slices using non-functional guanosine diphosphate (GDP) and GTP homologs and dynasore, a pan-dynamin blocker, but this has not yet been shown in dynamin knockout animals. This study also focused on activity-dependent endocytosis, whereas ligand-mediated endocytosis may underly the internalization of trophic factors (Xu et al., 2008). Additionally, GTP-independent endocytosis is not present at later ages (P13-14) (Yamashita et al., 2010). Most recently, knockout of the dynamin-related protein 1 (DRP1) in the calyx was accomplished using virally expressed Cre stereotaxic injection in the VCN at P1. This caused a drastic reduction in RRP, mitochondrial malformations, and smaller 
volume calyces (Singh et al., 2018). Interestingly, dynamin-mediated endocytosis has been shown to regulate BMP signaling for neurite outgrowth in the SH-SY5Y cell line in vitro and synaptic growth at Drosophila neuromuscular junctions (O'Connor-Giles et al., 2008; Hegarty et al., 2017). The relationship between dynamin and BMP signaling has yet to be studied in the developing calyx, however.

Conversely to the function of dynamin, the exocyst complex is a highly evolutionarily conserved octameric protein structure that functions to traffic vesicles to sites of fusion in a polarity-specific manner. The exocyst complex differentially shuttles vesicles to discrete areas of the cell for yeast budding, maintenance of apicobasal polarity in mammalian epithelial cells, intracellular reorganization in migratory cells, and neurite growth (Murthy et al., 2003; Pfenninger et al., 2003; Zajac et al., 2005; Polgar and Fogelgren, 2017). In Drosophila, the size of the neuromuscular junction postsynaptic membrane —-the subsynaptic reticulum (SSR)—is controlled in an activity-dependent manner by recruitment of exocyst complex secretory proteins (Sec)3/5/8/15 by the GTP-activated molecular switch RalA (Ras-related protein A).

Constitutively active RalA has also been shown to increase recruitment of Sec5 to dendritic spines in hippocampal neuron cultures, suggesting that this role may be conserved in mammals as well (Teodoro et al., 2013). Whether RalA and the exocyst complex serve this function in the PC has yet to be studied. In the calyx, overexpression of a dominant negative construct of Exo70 (green fluorescent protein (GFP)-Exo70 $\Delta \mathrm{C}$ ) - a exocyst complex constituent that functions to tether vesicles to the target membrane-using a recombinant adeno-associated virus (rAAV) causes defects in terminal morphology. Stereotaxic injection of rAAV in the VCN was performed on P2 rat pups, and calyces were assessed at P13 (large, non-fenestrated protocalyx) and P21 (fenestrated calyx). GFP-Exo70 $\Delta$ C calyces at P13 showed a significant deficit in volume compared to wild type that recovered by P21, though qualitatively there are significant morphological differences between P21 GFP-Exo70 $\Delta \mathrm{C}$ and wild type calyces. No 
electrophysiological differences between wild type, Exo70-overexpressing, and GFP-Exo70 $\Delta \mathrm{C}$ were found, suggesting the exocyst complex plays a role in terminal membrane growth and elaboration but is dispensable for synaptic vesicle fusion (Fig. 11G, Schwenger and Kuner, 2010). Unfortunately, due to variable expression times of the rAAV virus, no specific time point of Exo70 function can be gleaned from this study.

Most recently, the microRNA miR-96 has been shown to significantly influence the maturation of the calyx of Held. MicroRNAs are single-stranded RNA sequences that possess a "seed region" that binds to messenger RNAs with complementary sequences and lead to RNA degradation. Expressed exclusively in sensory systems of the brain, miR-96 is a member of a family of microRNAs including miR-182 and miR-183 (the miR-183 family) that influence hair cell differentiation and maturation, cochlear nucleus development, and photoreceptor development (Kuhn et al., 2011; Rosengauer et al., 2012; Zhang et al., 2015; Xiang et al., 2017). Mutations in the seed region of miR-96 are associated with progressive non-syndromic hearing loss, both in mice (diminuendo mutant) and humans (Lewis et al., 2009; Mencía et al., 2009). miR-96 expression is upregulated postnatally in the auditory brainstem, suggesting a role in development during this period (Rosengauer et al., 2012). Diminuendo (Dmdo) mutants show significant reductions in both MNTB volume ( 34\%) and PC size ( 22\%); interestingly, the difference in MNTB volume only becomes apparent at P4 and later. In addition, Dmdo mutant calyces and PCs maintain immature phenotypes into young adulthood (P25-30), both in terms of morphology and electrophysiological characteristics (Fig. 11H). Expression of both potassium channel $\mathrm{K}_{\mathrm{v}} 1.6$ and components of the voltage-gated BK potassium channel were downregulated in these mutants, suggesting a potential disruption in activity-dependent maturation of the CH:MNTB connection (Schlüter et al., 2018). However, microarray analysis of gene expression in the organ of Corti showed 96 genes differentially expressed in Dmdo mutants, along with hundreds of potential binding partners for the seed region of miR-96 (Lewis et al., 2009). 
Although this cell group is developmentally distinct from the CN and MNTB, the number of potential binding partners implies multiple potential affected targets in the Dmdo mouse.

\section{Development of Electrophysiological Characteristics of the Calyx of Held}

Although the electrophysiological properties of the $\mathrm{CH}$ :MNTB connection were not a focus of this research, it is important to understand the changes in the electrical properties of both preand post-synaptic cells that occur during the development of the calyx. To this end, a brief description of these changes will be detailed herein.

Neurotransmission at the $\mathrm{CH}$ :MNTB connection, as elsewhere in the nervous system, represents a cascade of events beginning and ending with the generation of an action potential. This AP, propagated by $\mathrm{Na}+$ and $\mathrm{K}+$ currents, causes opening of voltage-gated calcium channels in the presynaptic terminal. An influx of calcium leads to fusion of synaptic vesicles from the RRP, releasing neurotransmitter into the synaptic cleft that activates postsynaptic receptors. In turn, channels on the postsynaptic cell open, leading to an EPSC that-if sufficiently depolarizing - may trigger generation of a new AP in the postsynaptic cell. The speed at which these events can occur is of paramount importance in the $\mathrm{CH}$ :MNTB connection, as the computation of both ITD and ILD require precise temporal synchrony between contra- and ipsilateral signals. This requirement drives changes during development that tune the calyx to ensure high fidelity transmission. However, the early development of the calyx occurs in the absence of external auditory stimulus, as the ear canal does not open until the second postnatal week. Calyces in congenitally deaf mice $(d n / d n)$ also form correctly, with no obvious difference in morphology compared to wild type animals in either young (P8) or adult (P20) calyces (Fig. 12A, Youssoufian et al., 2008). Despite lacking sensitivity to sound, the developing auditory system is still active even in congenitally deaf animals due to spontaneous activity generated at the level of the inner hair cells. Release of adenosine triphosphate (ATP) by cells in Kölliker's organ-a transient epithelial structure in the cochlea that is transformed into 
the inner sulcus of the organ of Corti after hearing onset-causes bursts of APs to be fired by inner hair cells (IHCs) that propagate throughout the developing auditory circuitry and can be detected at the CH:MNTB connection (Fig. 12B, Tritsch et al., 2007; Youssoufian et al., 2008; Sonntag et al., 2009; Tritsch and Bergles, 2010; Dayaratne et al., 2014). This spontaneous activity is only detected in $\sim 50 \%$ of IHCs prior to P4 and increases in frequency and amplitude until it is abolished at the level of Kölliker's organ after hearing onset (Fig. 12C, Tritsch et al., 2007; Tritsch and Bergles, 2010; Sonntag et al., 2011). Although morphology is unaffected in $d n / d n$ animals, electrophysiological changes have been demonstrated: alterations in $\mathrm{Na}^{+}$and $\mathrm{K}^{+}$ conductances, hyperexcitability of PCs leading to firing of multiple APs on depolarization, increase in frequency and decrease in amplitude of miniature inhibitory postsynaptic currents (mIPSCs) and loss of tonotopically graded firing patterns (Leão et al., 2004a, 2004b, 2006a, 2006b). It is important to note, however, that these studies were almost exclusively performed in P12-14 animals, with the exception of examination of $\mathrm{Na}^{+}$conductance at $\mathrm{P} 7$ which showed no difference between $d n / d n$ and wild type animals (Leão et al., 2006a). Disruption of temporal precision of bursting activity in IHCs has been shown to distort the tonotopic map and interfere with synaptic refinement between the MNTB and LSO prior to hearing onset, but similar phenotypic results have yet to be shown for the $\mathrm{CH}$ :MNTB connection (Clause et al., 2014). Finally, mice lacking the $\mathrm{Ca}_{\mathrm{v}} 1.3$ subtype of the L-type calcium channel-a channel necessary for glutamate release from IHCs—show typical calyceal morphology and very similar electrophysiology to wild type animals at P8-10, despite having theoretically been deprived of spontaneous activity from the cochlea. Spontaneous activity from the VCN, even in the absence of afferent signaling from the cochlea, cannot be discounted as a potential source of activity, however (Erazo-Fischer et al., 2007; Hirtz et al., 2011). Further study will be necessary to determine if prehearing spontaneous activity is necessary for non-morphological aspects of $\mathrm{CH}:$ MNTB maturation. 
Both pre- and postsynaptic electrophysiological changes occur in tandem during development of the calyx of Held. One of the most pronounced changes in the presynapse is the sharpening of the action potential, with faster rise and decay times and a significantly reduced AP half-width but without a significant change in amplitude (Fig. 13A, Taschenberger et al., 2002; Fedchyshyn and Wang, 2005; Sonntag et al., 2011). One limiting factor in high-frequency transmission of action potentials is the time course of inactivation and recovery of voltage-gated sodium $\left(\mathrm{Na}_{\vee}{ }^{+}\right)$ channels. These channels consist of an $\alpha$-subunit and, in some cases, one or more $\beta$-subunits. Nine $\alpha$-subunit subtypes are known $\left(\mathrm{Na}_{\mathrm{v}} 1.1-\mathrm{Na}_{\mathrm{v}} 1.9\right)$, and these four-domain transmembrane channels can function in the absence of $\beta$-subunit binding (Ahern et al., 2016). In the MNTB, $\mathrm{Na}_{\vee} 1.6$ is increasingly expressed during development from at least P6 to P13. During this time, both inactivation and recovery times of $\mathrm{Na}^{+}$channels in the calyx decreases significantly. Interestingly, $\mathrm{Na}_{\mathrm{v}}{ }^{+}$channels are excluded from the calyx and reside on an elongated axonal heminode leading into the terminal; biophysical modeling of APs in a calyx with varying $\mathrm{Na}_{v}{ }^{+}$ conductance shows a positive correlation between $\mathrm{Na}_{v}{ }^{+}$conductance and half-width of the AP, suggesting that exclusion of $\mathrm{Na}_{v}{ }^{+}$channels from the calyx helps sharpen the AP waveform (Fig. 13B, Leão et al., 2005). $\mathrm{Na}_{v}{ }^{+}$conductance is also responsible for a depolarizing after potential (DAP) that occurs following an action potential in the calyx; following the initial $\mathrm{Na}^{+}$influx from opening of $\mathrm{Na}_{\mathrm{v}}{ }^{+}$channels, a secondary $\mathrm{Na}^{+}$influx occurs due to a subset of inactivated $\mathrm{Na}_{\mathrm{V}}{ }^{+}$ channels that pass through an "open" state before returning to a closed configuration. This depolarization peaks faster as development proceeds (P5-P12), may be mediated by the $\mathrm{Na}_{v}{ }^{+}$ $\beta_{4}$-subunit, and serves to increase both the sharpening of the AP and the reliability of firing at the calyx (see rightmost portion of curves in Fig. 13A, Hee Kim et al., 2010).

Between the initial depolarization and the DAP, voltage gated potassium $\left(\mathrm{K}_{\mathrm{v}}^{+}\right)$channels open, rectifying the depolarization and bringing the calyx back to near resting potential (approximately 
$-80 \mathrm{mV}$, Borst et al., 1995). $\mathrm{K}_{\mathrm{V}}{ }^{+}$channels consist of 4 a-subunits in either a homotetrameric or heterotetrameric configuration. There are currently 12 known $\mathrm{K}_{\mathrm{v}}{ }^{+}$channel families $\left(\mathrm{K}_{\vee} 1-\mathrm{K}_{\vee} 12\right)$, with multiple subtypes for each (Grizel et al., 2014). Two $\mathrm{K}_{\mathrm{v}}{ }^{+}$channel families $-\mathrm{K}_{\mathrm{v}} 1$ and $\mathrm{K}_{\mathrm{v}} 3$ are the dominant $\mathrm{K}_{\mathrm{V}}{ }^{+}$channels in the calyx, representing approximately $26 \%$ and $57 \%$ of the overall $\mathrm{K}^{+}$current respectively. The remaining $\mathrm{K}^{+}$current is mediated by the $\mathrm{Ca}^{2+}$-activated "big" potassium (BK) channel and a small, unknown current source (Ishikawa et al., 2003; Nakamura and Takahashi, 2007). Over a period (P7-P14) coinciding with the increase in density of $\mathrm{Na}_{\vee}{ }^{+}$ channels, both $\mathrm{K}_{\mathrm{v}} 1$ and $\mathrm{K}_{\mathrm{v}} 3$ channel densities increase approximately threefold (Fig. 13C, Elezgarai et al., 2003; Nakamura and Takahashi, 2007). Concurrently, $\mathrm{K}^{+}$current amplitude increased six-fold and rise time decreased three-fold for both channels. Despite the similarity in developmental changes, $\mathrm{K}_{\mathrm{v}} 1$ and $\mathrm{K}_{\mathrm{v}} 3$ channels have been shown to mediate different aspects of presynaptic signaling. $\mathrm{K}_{\mathrm{v}} 3$ channels ensure high-fidelity transmission at the calyx during highfrequency firing, as demonstrated by an increase in failure rates at P13-15 comparable to prehearing (P7-8) levels when these channels are blocked (Fig. 13D). An increase in $\mathrm{K}_{\mathrm{v}} 1$ channels, however, mediates the transition of calyx firing from tonic to phasic, shown by a return to tonic firing of the calyx at $\mathrm{P} 13-15$ when $\mathrm{K}_{\mathrm{v}} 1$ channels are blocked (Fig. 13E, Nakamura and Takahashi, 2007).

Faster depolarization and repolarization of the calyx during APs has a profound effect on the dynamics of calcium influx presynaptically, a process mediated by voltage-gated calcium channels (VGCCs). Like the $\mathrm{Na}_{\mathrm{V}}{ }^{+}$channel, VGCCs consist of a four-domain pore-forming transmembrane $\alpha_{1}$-subunit and multiple auxiliary subunits, of which the $\alpha 2 \delta$ - and $\beta$-subunits are most commonly found in neurons. Three classes of VGCCs have been reported $\left(\mathrm{Ca}_{\mathrm{v}} 1-3\right)$, each with a variety of subtypes. $\mathrm{Ca}_{\sqrt{ }} 2.1$ and $\mathrm{Ca}_{\sqrt{ }} 2.2(\mathrm{P} / \mathrm{Q}$ - and $\mathrm{N}$-type calcium channels, respectively) are most commonly found at synapses, and display a fast inactivation upon voltage-dependent 
activation (Nanou and Catterall, 2018). Both channel types mediate $\mathrm{Ca}^{2+}$ influx at the protocalyx from P4-P7, along with the small contribution of R-type $\left(\mathrm{Ca}_{\mathrm{v}} 2.3\right) \mathrm{Ca}^{2+}$ channels. From $\mathrm{P} 7-10$, the contribution of N-type VGCCs declines to undetectable levels, while P/Q-type channels increase (Fig. 14A, Iwasaki and Takahashi, 1998; Iwasaki et al., 2000). Despite this shift in VGCC composition, a substantial number of VGCCs $(60+)$ must still be activated for each synaptic vesicle to be released at P8-10, most likely owing to the distance from VGCCs to the RRP and the high concentration of $\mathrm{Ca}^{2+}$ necessary to elicit vesicle release (Borst and Sakmann, 1996). However, contrary to what might be expected, recruitment of VGCCs decreases as APs become more sharply tuned; both decreased rise and decay times contribute to this confinement of the $\mathrm{Ca}^{2+}$ transient, while the rate of decay has also been shown to influence VGCC kinetics. In spite of this, the quantal output per AP remains unchanged, suggesting an increase in coupling efficiency between $\mathrm{Ca}^{2+}$ influx and vesicle fusion (Fedchyshyn and Wang, 2005; Yang and Wang, 2006; Kochubey et al., 2009). Besides a shift in VGCC type, these developmental changes rely heavily on changes to the MAC (as discussed at length in the previous section on SAD kinase and RIM proteins) that shift the vesicle fusion mechanism from "microdomain" (many VGCC, both N- and P/Q-type, with loose coupling to vesicles) to "nanodomain" (vesicle fusion mediated by a small number of P/Q type VGCCs) (Fig. 14B, Fedchyshyn and Wang, 2005). In short, these changes include increased cluster size and VGCC number, an approximately twofold or greater increase in RRP, and decreased distance between VGCCs and synaptic vesicles (Fig. 14C, Taschenberger and von Gersdorff, 2000; Taschenberger et al., 2002; Fedchyshyn and Wang, 2005; Yang and Wang, 2006; Kochubey et al., 2009; Yang et al., 2010; Nakamura et al., 2015). Stronger vesicle-VGCC coupling is mediated in part by Septin5, one of 14 members of the filament-forming GTP/GDP-binding Septin family of proteins. Calyces in immature Septin5-knockout mice (P8-12) showed a twofold increase in vesicles located less than $5 \mathrm{~nm}$ from the AZ, marked increase in evoked EPSCs, 
a larger RRP, and increased resistance to the calcium buffer egtazic acid (EGTA); these characteristics were similar to those of more mature calyces (P16-19), but significantly different from age-matched wild type animals. Septin5 appears to act as a component of a barrier that keeps many vesicles from associating closely with the AZ early in development but is reorganized to permit this association as the calyx develops (Fig. 14D, Yang et al., 2010). Further study will be necessary to determine if Septin5 represents the filamentous structure that has been identified in MACs.

VGCC activity is also modulated by the inhibitory effects of serotonin (5-HT) and adenosine, and this inhibition declines as development proceeds (P5-P14) (Kimura et al., 2003; Mizutani et al., 2006). Later in development (P14-P19), gamma-Aminobutyric acid (GABA) also inhibits VGCCs through $\mathrm{GABA}_{\mathrm{B}}$ receptors, but their function in early postnatal development has yet to be described (Takahashi et al., 1998). Contrary to their canonical function, both GABA and glycine have also been found to weakly depolarize the developing calyx and enhance neurotransmitter

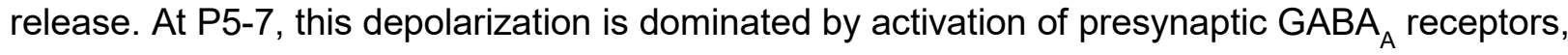
while at P12 the effect of GABA is negligible, and this depolarization is dominated by glycine (Fig. 14E). Glycine spillover from inhibitory terminals has been shown to weakly depolarize the calyx by activating $\mathrm{Cl}^{-}$channels that subsequently cause $\mathrm{P} / \mathrm{Q}$-type $\mathrm{Ca}^{2+}$ channels to open, increasing internal $\mathrm{Ca}^{2+}$ concentration and the probability of vesicle release. Although the exact mechanism of action for GABA depolarization hasn't been completely detailed, this process is also mediated by $\mathrm{Cl}^{-}$, suggesting it may function similarly to glycine receptor activation (Turecek and Trussell, 2001, 2002; Awatramani et al., 2005a). Further characterization of the changes in intraterminal and intracellular $\mathrm{Cl}^{-}$levels throughout development of the $\mathrm{CH}$ :MNTB is needed to understand how this modulation of electrical potential is regulated. 


\section{Principal Cell Growth and Electrophysiological Maturation}

During the developmental window of calyx growth, substantial changes in the principal cells of the MNTB also occur. Although multiple studies have examined PC morphology in the adult animal using stained sections, light microscopy, and electron microscopy (EM), there exists a paucity of information regarding the morphological changes in this cell type accompanying calyceal development (Morest, 1968b; Jean - Baptiste and Morest, 1975; Ollo and Schwartz, 1979; Banks and Smith, 1992; Kuwabara and Zook, 1992; Kil et al., 1995). Based on EM images—chosen for their high resolution and lack of bias based on staining technique or antibody — from Hoffpauir et al., some morphological features of these cells can be gleaned (Hoffpauir et al., 2006). Immature MNTB PCs (P0) appear closely apposed to one another and bipolar in morphology with an eccentric nucleus. The long cell axis parallel to the long axis of the cell nucleus measures approximately $16 \mu \mathrm{m}$ in diameter, and the short axis is approximately 10 $\mathrm{um}$ in diameter (Fig. 15A). This morphology appears to persist until P2 (Fig. 15B). By P3, cells appear more spheroid (Fig. 15C), and a reversal of primary axes is evident at P4, with the axis parallel to the long axis of the cell nucleus appearing unchanged in diameter but the

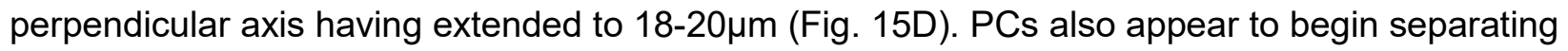
from one another at P2, with substantial space between cells apparent by P4 (Fig. 15A-B, Hoffpauir et al., 2006). Estimations of cell surface area based on calculations from cell perimeter measurements in serial section electron micrographs show an increase in surface area from $377 \pm 44 \mu \mathrm{m}^{2}$ at $\mathrm{P} 0$ to $498 \pm 75 \mu \mathrm{m}^{2}$ at $\mathrm{P} 4$, with the largest increase $\left(\sim 60 \mu \mathrm{m}^{2}\right)$ from P3-P4. No appreciable difference in cell size or shape is evident from P4-P10 in EM images from multiple studies (P8-10 (Müller et al., 2009), P9 (Sätzler et al., 2002), P10 (Kil et al., 1995)), and the estimated long $(\sim 20 \mu \mathrm{m})$ and short $(\sim 16 \mu \mathrm{m})$ axis lengths agree with estimates of cell size in the adult MNTB $(22.4 \pm 3.01 \mu \mathrm{m} \times 15.8 \pm 2.42 \mu \mathrm{m}$ in rat (Banks and Smith, 1992); $20 \mu \mathrm{m}$ average diameter in cat (Morest, 1968a); 16.4 $\mu \mathrm{m}$ average diameter in mouse (Ollo and Schwartz, 
1979)). PC size is tonotopically graded, with smaller cells medially and larger cells laterally (Fig. 15E, Pasic et al., 1994; Weatherstone et al., 2016). Intriguingly, PC size appears regulated by activity; when activity is abrogated due to cochlear ablation or blockade of the IIX nerve by application of tetrodotoxin (TTX), PC soma size is reduced by $\sim 15 \%$, but returns to normal upon removal of the nerve block (Jean-Baptiste and Morest, 1975; Webster, 1983; Pasic et al., 1994). The MNTB tonotopic PC size gradient may also be mediated by the plasma membrane calcium ATPase pump (PMCA2, Fig. 15F), as this size gradient is abolished in deafwaddler mutant mice either lacking PMCA2 or possessing mutated PMCA2 with greatly reduced function (Fig. 15G, Weatherstone et al., 2016).

In concert with electrophysiological changes in the calyx, the PC undergoes corresponding changes in excitability. As mentioned previously, evoked EPSCs in the PC can be detected as early as E17 through stimulation of the AVCN in a whole-head slice preparation (Hoffpauir et al., 2010). Similar to the calyx, the neurotransmitters glutamate, GABA, and glycine can all elicit depolarization to different degrees in the PC early in development (P0-P7); at these early ages, glutamate causes a "fast" current response, while the "slow" response is elicited by GABA (Fig. 16A, Awatramani et al., 2005b; Hoffpauir et al., 2010; Rusu and Borst, 2011). Principal cells also undergo a shift in response to these canonically inhibitory molecules from predominantly $\mathrm{GABA}_{\mathrm{A}}$-receptor mediated to glycine receptor mediated; however-unlike the calyxGABAergic response persists in P13-P15, but at a weaker amplitude (Awatramani et al., 2005b). From E17-P1, spontaneous EPSCs are evoked in at least $20 \%$ of PCs, most likely through presynaptic spontaneous release of vesicles independent of an AP (Fig. 16B, Hoffpauir et al., 2010; Rusu and Borst, 2011). Interestingly, this spontaneous release has been shown to be at least partially independent of VGCCs or SYT-2 ( 40\%), though the exact mechanism behind this release has not yet been codified (Dai et al., 2015). Hoffpauir et al. showed a significantly higher spontaneous EPSC amplitude evoked by glutamate versus GABA (- 
$61.0 \pm 18.3 p A$ vs. $-41.53 \pm 15.0 p A)$ in $\mathrm{E} 17-\mathrm{P} 0$ mice, with glutamatergic events occurring three times more often (Fig. 16A, Hoffpauir et al., 2010). Subsequently, Rusu and Borst demonstrated a similarity in amplitude between these two currents $(-39.6 \pm 1.7 p A$ for glutamate, $-32 \pm 1.8 p A$ for GABA) with less frequent GABAergic EPSCs (10\%) (Rusu and Borst, 2011). These measurements were done at P0 in the rat, however, which normally lags at least one day behind the mouse in terms of development, so it is possible that these currents represent a more immature state than those measured in the mouse (Clancy et al., 2001). Spontaneous activity decreases by P2-3 (<5\%) and is undetectable in P4 and later cells (Rusu and Borst, 2011).

Glutamatergic response at the PC is mediated by a combination of AMPA and N-methyl-Daspartate (NMDA) receptors (Fig. 16C, Forsythe and Barnes-Davies, 1993; Futai et al., 2001; Taschenberger et al., 2002; Hoffpauir et al., 2006; Rusu and Borst, 2011). AMPA receptors (AMPARs) are composed of four subunits (GluR1-4) that, when combined in different combinations, create unique AMPAR subtypes with distinct functions. These receptors are predominantly responsible for the fast excitatory post-synaptic potential (EPSP) response to glutamate in most neurons (Song and Huganir, 2002). In contrast, NMDA receptors (NMDARs) both open and close slower than AMPARs, creating slower, more prolonged EPSPs more likely to sum temporally and thus limit the rate of AP firing (Joshi et al., 2007). NMDARs are tetrameric, being composed of two copies of an NR1 subunit and either two NR2 or NR3 subunits. These receptors also require depolarization of the postsynaptic membrane to remove the $\mathrm{Mg}^{2+}$ that blocks the channel formed by the tetramer (Furukawa et al., 2005). Combining these two properties, NMDARs therefore work as coincidence detectors, needing both postsynaptic depolarization and binding of glutamate together in order to function; when depolarization and glutamate binding are separated temporally by 3.5 seconds, the $\mathrm{Mg}^{2+}$ block is removed significantly more slowly and the amplitude of NMDAR-mediated depolarization is 
reduced by more than $50 \%$ compared to a separation of only 10 milliseconds (Mayer et al., 1984; Kampa et al., 2004). Interestingly, co-binding of glutamate and glycine for some NR1/NR2 combinations can enhance the speed of NMDAR response, suggesting a transition from GABA to glycine release at PCs may have more than just an inhibitory role (Johnson and Ascher, 1987; Furukawa et al., 2005). Both AMPAR- and NMDAR-mediated currents increase early in development, with AMPAR currents increasing by a factor of 30 from P1-4 and NMDAR currents increasing 17-fold over the same time. This EPSC increase is most evident at the P3-P4 transition, when some small terminals grow rapidly into protocalyces. Additionally, the contribution of AMPAR currents to the EPSC increases from P1-4 (ratio of AMPAR to NMDAR currents: 0.45 at P1, 0.83 at P4) (Fig. 16D). APs evoked in the PC during this postnatal time period arise from summation of EPSCs from multiple small inputs, rather than a single large terminal (Hoffpauir et al., 2006; Rusu and Borst, 2011; Sierksma et al., 2017). However, both resting membrane potential (RMP) and membrane resistance of the PC decrease from E17-P4 ( $-55 \mathrm{mV}$ to $-72 \mathrm{mV}$ and $1.75 \mathrm{G} \Omega$ to $\sim 0.4 \mathrm{G} \Omega$, respectively), increasing the threshold current necessary to trigger an AP; the smallest inputs to PCs were capable of eliciting APs in $50 \%$ of cases at E18-P0, but by P2-3 none of the smallest inputs could drive their postsynaptic partners (Fig. 16E, Hoffpauir et al., 2010). This synchrony of development-with increased postsynaptic membrane resistance and hyperpolarity met with larger drive presynaptically—may represent a selectivity process by which small supernumary inputs are negatively selected for and removed (Hoffpauir et al., 2010; Rusu and Borst, 2011; Sierksma et al., 2017).

Changes in PC firing, EPSC and AP waveform patterns occur early in development and continue to well beyond the onset of hearing. PCs transition from an almost exclusively tonic firing pattern at P0 to nearly all phasic firing by P6 (Fig. 17A, Hoffpauir et al., 2010). Kv1.1 and Kv1.2-two low-threshold voltage gated $\mathrm{K}^{+}$channel $\alpha$-subunits expressed in the PC-have been shown to limit the burst firing of APs from the PC in P5-15 rats, allowing for phasic firing by 
opening rapidly but closing slowly (Brew and Forsythe, 1995; Dodson et al., 2002). These channel subunits were shown to be highly expressed in the proximal PC axon, allowing them to have a profound effect on AP kinetics (Fig. 17B, Dodson et al., 2002). In mice, messenger RNA (mRNA) expression of these two channels in the PC increases from P0.5 to P8 (Kv1.2) or P14 (Kv1.1), and blocking these channels causes them to return to a tonic firing phenotype, both in P8-14 rats and in P2-3 cells that have already established phasic firing in mice (Fig. 17C, Dodson et al., 2002; Hoffpauir et al., 2010). A reduction in postsynaptic depression and failure rate, as well as an increase in phase locking, all occur during the second postnatal week. At P7, PCs fire faithfully to presynaptic stimulation up to $20 \mathrm{~Hz}$, which $20-100 \mathrm{~Hz}$ stimulation causes excess firing due to summation of EPSP tails, and $100 \mathrm{~Hz}$ stimulation causes AP blockage due to EPSP plateau and synaptic depression. P15 cells show higher fidelity, fewer improper AP firings, and no loss of APs at $100 \mathrm{~Hz}$, and cells at P27 fire faithfully at $400 \mathrm{~Hz}$ (Futai et al., 2001). Some cells at P14 were found to already be able to fire with high accuracy at stimulation frequencies up to $600 \mathrm{~Hz}$ (Taschenberger and von Gersdorff, 2000). In addition to maturation in timing, a decrease in failure rate is also evident across this developmental time period; PCs at P5-P8 showed failure of AP firing following EPSPs at a rate of $\sim 60 \%$, reducing to $\sim 40 \%$ at P8-9, $1.4 \%$ at P12, and becoming undetectable at P14 (Fig. 17D, Joshi and Wang, 2002; Sonntag et al., 2011). High voltage, non-inactivating potassium channels $K_{v} 3.1 a$ and $K_{v} 3.1 b$ are at least partially responsible for these developmental changes. Microarray analysis of the MNTB from P0-14 shows a slight increase in $\mathrm{K}_{\mathrm{v}} 3.1$ RNA expression, though this may not translate directly into increased protein expression (Kolson et al., 2016). Several studies in other nearby brain regions have examined expression of this channel family across development as well. $\mathrm{K}_{\mathrm{v}} 3.1 \mathrm{a}$ is expressed as early as E17 in rat brains-most prominently in the cerebellum-and expression of this subtype increases until P10. Expression of $\mathrm{K}_{v} 3.1 \mathrm{~b}$ is lower and shows less of an increase during development, but the pattern of expression for both channel subtypes in the adult are 
very similar (Perney et al., 1992). In the cerebellum and inferior colliculus, $\mathrm{K}_{\mathrm{v}} 3.1 \mathrm{~b}$ increases significantly from P8-40 in an activity-dependent manner (Liu and Kaczmarek, 1998a, 1998b); if this same increase occurs in MNTB, the timing coincides well with the shift in PC firing properties. $\mathrm{K}_{\mathrm{v}} 3.1$ channels are expressed along a tonotopic gradient in the MNTB, with the highest expression in the medial, high frequency portion (Li et al., 2001; von Hehn et al., 2004). This tonotopic gradient is abolished in 8 month old C57BL/6 mice, a mouse model that loses high-frequency cochlear hair cells between 3-6 months of age (von Hehn et al., 2004). Thus, an activity-dependent and frequency-specific maintenance of $\mathrm{K}_{v} 3.1$ channels exists in PCs of the MNTB. When $\mathrm{K}_{\mathrm{v}} 3.1$ channels are either blocked using TEA (tetraethylammonium) or knocked out genetically, PCs fail to be able to follow high frequency stimulus patterns $(200-400 \mathrm{~Hz})$, have significantly higher failure rates that increase with frequency, and reduced amplitude of EPSPs associated with synaptic depression (Fig. 17E, Brew and Forsythe, 1995; Wang et al., 1998; Macica et al., 2003). Additionally, $\mathrm{K}_{\mathrm{v}} 3.1 \mathrm{~b}$ channel activity can be diminished through serine phosphorylation by protein kinase $\mathrm{C}(\mathrm{PKC})$, and high frequency stimulation causes a decrease in phosphorylated $\mathrm{K}_{\mathrm{v}} 3.1 \mathrm{~b}$. PCs may therefore be able to tune their response to stimulation by PKC-dependent $\mathrm{K}_{\mathrm{v}} 3.1 \mathrm{~b}$ phosphorylation, allowing for fast high-amplitude AP firing across a range of frequencies (Song et al., 2005).

Modifications in both AMPARs and NMDARs also have a profound influence on the ability of PCs to faithfully follow high-frequency stimuli. As mentioned previously, the slow rate of NMDAR-evoked EPSC is a rate-limiting step for high-frequency AP firing. However, blocking of NMDARs in immature PCs using (2R)-amino-5-phosphonovaleric acid (D-APV or AP5) eliminates temporal summation, reduces postsynaptic depression during high frequency firing, and confers upon these cells a firing pattern typical of P16-19 PCs (Fig. 18A, Futai et al., 2001; Joshi et al., 2007). From P6-P14, NMDAR-mediated EPSC amplitude decreases by $\sim 50 \%$ 
( $15 \mathrm{nA}$ to $\sim 7 \mathrm{nA}$ ) and NMDAR decay time also shortens significantly (time constant for single exponential fit to data: $7.06 \pm 0.6 \mu$ s to $4.21 \pm 0.19 \mu \mathrm{s}$ ) (Fig. 18B, Taschenberger and von Gersdorff, 2000). Temporal summation of NMDAR EPSCs—indicative of slow NMDAR kinetics—is present as late as P12, but absent by P18 (Fig. 18A, Joshi et al., 2007). NMDAR EPSCs also diminish even further by P18 to $0.3-0.5 \mathrm{nA}$ (Steinert et al., 2010). Taken together, these findings suggest a substantial change in NMDARs following the onset of hearing. This hypothesis is strengthened by the fact that P7 animals that have undergone bilateral cochlear ablation show higher levels of mRNA from NMDAR subunits NR2A and NR2B, larger NMDAR-mediated EPSCs, and a greater failure rate at $100 \mathrm{~Hz}$ stimulation at $\mathrm{P} 14$ than age-matched sham animals (Futai et al., 2001). Three factors may contribute to the changes seen in NMDAR-EPSCs in PC development: decreased numbers of NMDARs, changes in NMDAR subunit composition, and a shift in the $\mathrm{Mg}^{2+}$ dissociation constant. A decrease in total mRNA and protein for NMDAR subunits NR1, NR2A, and NR2B from P10-P27 in mice is indicative of an overall reduction in NMDAR production (Futai et al., 2001). An increase in activity upon onset of hearing may also decrease perisynaptic NMDARs; paired calyx stimulation and PC depolarization leads to coactivation of NMDARs and metabotropic glutamate receptors (mGluR2/5), resulting in clatherindependent endocytosis of NMDARs outside of the synapse (Fig. 18C, Joshi et al., 2007). The constituent subunits of NMDARs influence their kinetics and sensitivity to $\mathrm{Mg}^{2+}$, but the makeup of NMDARs across development is still a matter of debate due to conflicting evidence (CullCandy et al., 2001). Based on a transition to faster kinetics, it has been suggested that a shift from NR2B subunits to the faster NR2A subunits is most likely occurring after hearing onset (Taschenberger and von Gersdorff, 2000). Although Futai et al. show a decrease in both NR2A and NR2B subunit mRNA and protein over this period in mice, NR2B protein decreases less than NR2A, contrary to what would be expected (Fig. 18D, Futai et al., 2001). Expression does not necessarily translate into incorporation into the NMDAR, however, so it is still possible that this subunit transition occurs. Contrary to these findings, Steinert et al. show a significant 
increase in mRNA for the NR2A and NR2B subunits of NMDAR in rats from P10 to P21, along with consistently higher peak amplitudes of NMDAR EPSCs compared to mice. This suggests there may be a species-specific difference in both subunit regulation and overall protein expression in rats and mice for this channel (Fig. 18E, Steinert et al., 2010). Finally, PCs in mice show an increased sensitivity to $\mathrm{Mg}^{2+}$ concentration $\left(\left[\mathrm{Mg}^{2+}\right]\right)$ from $\mathrm{P} 6-12$, causing a greater block in NMDAR-mediated EPSC; this increased $\left[\mathrm{Mg}^{2+}\right]$ sensitivity-along with faster NMDA kinetics-is indicative of a reduction in the slow, $\mathrm{Mg}^{2+}$-sensitive NR2C/D subunits (Cull-Candy et al., 2001; Joshi and Wang, 2002). $\left[\mathrm{Mg}^{2+}\right]$ sensitivity appears to decline between P12-14 in mice, and a similar decrease is seen from P11-18 in rats. This may represent a transition from the slower NR2B subunit to the faster but more $\mathrm{Mg}^{2+}$-sensitive NR2C; indeed, this appears to be the case in PCs in rats, but has yet to be shown in mice (Joshi and Wang, 2002; Steinert et al., 2010).

Similar to NMDAR, changes in AMPAR kinetics contribute to the ability of PCs to phase lock to high frequency firing from the calyx of Held without loss of amplitude (Joshi et al., 2004). The AMPAR-evoked EPSC in mice increases in amplitude by a factor of 3 from P5-P14, with little change after this. During this time, rise and decay times for this EPSC decrease by $\sim 55 \%$ and $\sim 70 \%$, respectively. (Fig.s 16D and 19A, Futai et al., 2001; Joshi and Wang, 2002). In rats, AMPAR kinetics change similarly to mice, however the peak amplitude of AMPAR-mediated EPSCs does not change significantly from P5-P14 (Fig. 18B, Taschenberger and von Gersdorf, 2000). The sharpening of AMPAR EPSCs is most likely a result of a combination of multiple factors. One method for shortening decay time is receptor desensitization, where chronic exposure to high concentrations of neurotransmitter reduces the response from receptors in the postsynaptic cell. Kynurenate (KYN) and cyclothiozide (CTZ), both blockers of AMPAR desensitization, abolished AMPAR desensitization at P5-8 but had little effect on EPSCs at P1214 in both mice and rats (Joshi and Wang, 2002; Taschenberger et al., 2002; Koike-Tani et al., 
2005). Desensitization of AMPARs at younger ages is due in part to an immature ability to effectively clear glutamate from the synaptic cleft, as the decay time of evoked EPSCs with large amounts of glutamate release are more affected by CTZ desensitization than spontaneous EPSCs (Fig. 19B, Koike-Tani et al., 2005). This suggests that desensitization of AMPARs may play a substantial role in tuning AMPAR-evoked EPSCs at younger ages but has less impact after onset of hearing due to increased glutamate clearance. AMPAR-mediated EPSCs are suppressed in P8-10 rats when glutamate transporters are blocked, but not in P16-18 animals (Renden, 2005). As these time points bracket both the onset of hearing and the fenestration of the protocalyx, it is possible that glutamate clearance in the $\mathrm{CH}$ :MNTB synaptic cleft switches from glutamate transporter-dominated to diffusion-dominated (Koike-Tani et al., 2005; Renden, 2005). In addition to desensitization, faster deactivation of the AMPAR channel may contribute to the reduction in decay time in the EPSC. Short ( 1ms) applications of glutamate to PCs showed a significant decrease in the time constant for deactivation from P6-14 in both mice $(1.35 \pm 0.12$ vs $0.81 \pm 0.07 \mathrm{~ms})$ and rats $(1.72 \pm 0.16$ vs $0.81 \pm 0.07 \mathrm{~ms})$ (Joshi et al., 2004 ; KoikeTani et al., 2005). AMPAR deactivation kinetics are determined by their subunit makeup, with GluR1 subunits being the slowest to deactivate and GluR3/4 having rapid deactivation kinetics (Traynelis et al., 2010). In agreement with this, GluR1 decreases in expression in both mice (P5-P18) and rats (P7-P21), while both GluR3 and GluR4 increased over the same time period (Fig. 19C, Caicedo and Eybalin, 1999; Joshi et al., 2004; Koike-Tani et al., 2005, 2008). Immunogold-labeling in the P9 and adult rat also shows very little GluR1 at the synapse in comparison to GluR2/3 or GluR4 (Hermida et al., 2006, 2010). Very little GluR2 expression is evident from P5-18 in the mouse, and despite an apparent increase in GluR2 mRNA in the rat from P7-21, immunohistochemical analysis of MNTB in rats shows no increase in GluR2 from P4-30. This is of particular importance, as inclusion of the GluR2 subunit conveys an impermeability to $\mathrm{Ca}^{2+}$ ions on the AMPAR channel. (Caicedo and Eybalin, 1999; Joshi et al., 2004; Koike-Tani et al., 2005, 2008). In P16-19 mice, global knockout of GluR4 causes 
decrease in AMPAR-EPSC amplitude, increase in both rise and decay time, a 10-fold increase in AP failure rate, and substantial alterations in ABR amplitude and latency compared to wildtype controls. In contrast, GluR3-knockout mice showed only a modest increase in decay time constant, but no change in either amplitude or rise time in AMPAR-EPSC (Fig. 19D). Although deactivation time was not assessed in this study, loss of GluR4 exacerbates desensitization and increases short-term depression, suggesting that this AMPAR subunit is indispensable for maintaining proper firing properties of the PC (Yang et al., 2011). GluR subunits may also be alternatively spliced, resulting in either a "flip" or "flop" variant (Boulter et al., 1990; Sommer et al., 1990; Lomeli et al., 1994). Although similar in deactivation kinetics, the "flop" variant of these subunits typically has a much shorter desensitization time constant than their "flip" counterparts (Traynelis et al., 2010). A developmental shift from "flip" to "flop" GluR mRNA in the MNTB is apparent in rats after onset of hearing, with "flop" variants increasing from $83.6 \pm 4.9 \%$ at $\mathrm{P} 7$ to $94.7 \pm 2.0 \%$ at P14 (Fig. 19C, Koike-Tani et al., 2005). It is important to note, however, that changes in GluR variants have not been quantified in mice, and mRNA levels are not necessarily indicative of levels of protein expression.

\section{Glia in MNTB development}

(section reproduced-with minor edits—from "Perinatal Development of the Medial Nucleus of the Trapezoid Body", The Oxford Handbook of the Auditory Brainstem, 2018, with permission under the "After Publication Rights Granted" section of the Author Reuse and Self-Archiving Policy of Oxford University Press,

\section{https://global.oup.com/academic/rights/permissions/autperm/?cc=gbandlang=enand)}

Neuroglia in the MNTB, as in other brain regions, serve a diverse and important array of functions. As in the cerebral cortex and cerebellum, glial cells in the brainstem are believed to be derived from the ventricular neuroepithelium, from which radial glial processes project to create a "scaffold" that may be the basis for later neural organization (Van Hartesveldt et al., 
1986; Ariel Gomez et al., 1990; Parnavelas, 1999; Buffo and Rossi, 2013). Both astrocytes and oligodendrocytes are present in the MNTB as early as P0 in mice and rats, with microglia appearing in substantial numbers only after P6 (Fig. 20A, Dinh et al., 2014; Saliu et al., 2014). Using 5-ethynyl-2'-deoxyuridine (EdU) injections of pregnant rat dams, Saliu et al. showed that glial progenitor cells in the rat MNTB begin to divide prenatally (E19 or earlier), rapidly increase their rate of division until around the onset of hearing (approximately P12), and then slow their division exponentially until barely distinguishable at P18. EdU-labeled cells later stain positive for the commonly used astrocyte marker, $\mathrm{S} 100 \beta$. This timeline of glial proliferation occurs in synchrony with the developmental timeline of the $\mathrm{CH}$ terminal (Saliu et al., 2014). Although $\mathrm{S} 100 \beta$ is often used as a marker for astrocytes, this protein is also expressed in neural/glial antigen 2 (NG2) OPCs (Hachem et al., 2005). Therefore, proliferating glia in the MNTB may potentially assume either an oligodendrocytic or astrocytic fate (Hamori et al., 1997; Buffo and Rossi, 2013).

Both astrocytes and OPCs participate in a multi-partite synaptic system in the MNTB that is only partially understood. Light and ultrastructural analyses of the developing MNTB have shown that vellous processes, either from astrocytes or OPCs, occupy non-innervated territory on the principal cell surface, surround growing terminals, and interpose between the principal cell and terminals (Fig. 15A (black arrows), Jean - Baptiste and Morest, 1975; Elezgarai et al., 2001; Hoffpauir et al., 2006; Reyes-Haro et al., 2010; Dinh et al., 2014). Dinh et al. showed that vellous processes, present as early as P0 in mice, express $\mathrm{S} 100 \beta$, aldehyde dehydrogenase 1 family member L1 (ALDH1L1), or glial fibrillary acidic protein (GFAP), though the pattern of expression differs for each protein. From P0 to at least P23, ALDH1L1 glial processes associate with MNTB principal neurons and, later in development, the growing calyx. Processes expressing $S 100 \beta$ are similarly associated with these structures, though the processes were found to be more homogenously distributed throughout the MNTB. GFAP shows no expression 
within the MNTB at P0 and expression only in glial processes within the MNTB from P6 to P24. No GFAP cell bodies are found present in the MNTB at any age studied (Fig. 20B, Dinh et al., 2014). However, the extent of overlap in expression of these proteins in these processes has yet to be determined. A single astrocyte or OPC may also contact multiple principal cells, opening the possibility for regulation of network-level organization by these cells. Vellous processes also contain mGluR2/3 (P0-24 rats, P6-18 mice) and transporters (EAAT1[GLAST], EAAT2[GLT-1], P10-15 rats), allowing them to respond to glutamate release from the $\mathrm{CH}$, take up excess glutamate, and prevent glutamate receptor saturation in the immature calyx (Fig. 20C, Elezgarai et al., 2001; Renden, 2005; Uwechue et al., 2012). As the calyx matures and fenestrates, the risk of receptor saturation decreases, glutamate uptake by glial cells is less necessary, and expression of mGluR2/3 decreases in these processes (Elezgarai et al., 2001; Renden, 2005). Astrocytes may also influence synaptic refinement through gliotransmission and the induction of neuronal slow inward currents ( $\mathrm{nSICs);} \mathrm{repeated} \mathrm{stimulation} \mathrm{of} \mathrm{midline} \mathrm{axons}$ causes astrocytes to release both glutamate and D-serine that, together, coactivate NMDA receptors on the principal cell and induce nSICs (Fig. 20D, Reyes-Haro et al., 2010). In contrast, direct, AMPA receptor-mediated synaptic connections are established between the $\mathrm{CHs}$ and OPCs, allowing these cells to receive synchronized input (Fig. 20E, Müller et al., 2009). Glutamatergic input to NG2 OPCs has generally been shown to inhibit differentiation of these cells into more mature oligodendrocytes (Gallo et al., 1996; Yuan et al., 1998; Káradóttir and Attwell, 2007). However, it has been demonstrated more recently in the rat corpus callosum that the frequency of stimulation has a direct influence on OPC proliferation and differentiation, with $5 \mathrm{~Hz}$ stimulation increasing OPC differentiation and $25 \mathrm{~Hz}$ and $300 \mathrm{~Hz}$ stimulation increasing OPC proliferation (Nagy et al., 2017). As activity can influence both GBC axon diameter and myelin thickness (Sinclair et al., 2017), and as oligodendrocyte proliferation is the major contributor to cell proliferation during calyx development from P3-P6 (Brandebura et al., 2018), it is intriguing to think that changes in firing patterns may allow terminals to regulate their own 
oligodendrocyte differentiation and, therefore, their own axon myelination (Sinclair et al., 2017; Brandebura et al., 2018). The studies of both astrocyte gliotransmission and $\mathrm{CH}$ :OPC synapses were performed in 8-10 day old mice, after monoinnervation has already been established. Future studies will need to focus on earlier postnatal time points to elucidate the reciprocal effects that neuron-glial interactions may have on development of the MNTB.

\section{Competition in Calyx Development}

One of the most contentious questions regarding the development of the calyx of Held is that of competition. In other systems, such as the neuromuscular junction, climbing fiber:Purkinje cell connection, and the dorsolateral geniculate nucleus, supernumerary inputs form early in development and-through a process of competition-are pruned to either one or a small few inputs (Lee et al., 2002; Kasthuri and Lichtman, 2003; Hashimoto et al., 2009). However, while these systems can take 1-2 weeks or more to resolve competition, monoinnervation of the PC appears to occur in as little as 48 hours (Hoffpauir et al., 2006, 2010). Conflicting evidence exists for whether the terminal that eventually forms the calyx is established early in isolation (i.e., without competitors) or emerges after a growth process marked by competition with other calycigenic terminals. Although studies in the rat using either Dil or dextran labeling of VCN axons claim an absence of multiple large terminals on single PCs during development (Fig. 21A), two important caveats to these findings must be considered (Kandler and Friauf, 1993; Rodríguez-Contreras et al., 2006). First, these studies were carried out in P4 or older animals, after monoinnervation has largely been established (Kandler and Friauf, 1993; Hoffpauir et al., 2006, 2010; Rodríguez-Contreras et al., 2008; Soria Van Hoeve and Borst, 2010; Xiao et al., 2013). Second, the methods used only label a subset of axons, which is advantageous to examining the branching of axons and structure of terminals in isolation but leaves open the possibility that large terminals converging onto single PCs may have been missed.

Electrophysiological characterization of converging terminals using a minimum stimulation 
protocol from E18-P4 shows PC innervation at P4 by either a terminal capable of evoking an EPSC much larger $(>1 \mathrm{nA})$ than its next largest counterpart $(8 / 20)$, or two terminals with similar EPSC amplitudes (6/20) (Hoffpauir et al., 2010). The remaining cells (6/20) possessed only inputs with small EPSC values (Fig. 21B). It is possible that these cells represent an immature set of P4 cells — the recorded terminals appear much like those found at P3—but may also exemplify a problem endemic to slice recordings in the MNTB: because of the long path that GBC axons must take to reach their target cells in the contralateral MNTB, there is a high likelihood that a substantial fraction of axons are cut during slicing. Additionally, placement of the stimulating electrode relative to axons at the midline may lead to axons distant from the electrode not being activated. Minimal stimulation protocols are also prone to underestimating innervation due to terminals with similar thresholds that produce an additive EPSC, although this was partially controlled for by having multiple stimulation protocols of different voltage step sizes (Hoffpauir et al., 2006, 2010). Reconstructions from serial section electron microscopy provide the most accurate depiction of innervation of the PC: they are unbiased by specific antibodies, capture all inputs to the cell unlike Dil or dextran labeling, are capable of extremely high resolution in the imaging plane $(\sim 4-10 \mathrm{~nm})$ and are not limited by the diffraction limit as is confocal microscopy (Denk and Horstmann, 2004; Briggman and Bock, 2012; Horstmann et al., 2012). P4 reconstructions of PCs and associated large terminals show that $11 \%(2 / 19)$ of cells are innervated by two large terminals (Fig. 21C, Hoffpauir et al., 2006). Although this study was, again, done after resolution to monoinnervation for most PCs, it provides compelling evidence that PCs may receive multiple large inputs. Unfortunately, these reconstructions do not contain enough of the terminal axon to determine if these terminals emerge from separate axons or collaterals from the same axon. The rate of single GBC axons giving rise to two calyces is unclear, though studies have shown this phenomenon at many developmental time points and across a range of species; Rodríguez-Contreras et al., for example, found in the rat that $18 \%$ or more of GBC axons branched to form multiple calyces, irrespective of age (Morest, 1968a, 
1968b; Spirou et al., 1990; Kuwabara et al., 1991; Smith et al., 1991; Kandler and Friauf, 1993; Rodríguez-Contreras et al., 2006). While some calyceal axons bifurcate close to the PC and form seemingly separate calyces, axon collaterals that produce additional calyces have been identified as far as $50 \mu \mathrm{m}$ from the calyx, with significantly fewer at distances of $100 \mu \mathrm{m}$ or greater (Fig. 21D, Rodríguez-Contreras et al., 2006). These dual calyces are invariably located near to one another, most likely owing to frequency matching of GBCs and PCs to maintain tonotopy, though techniques used to examine these calyces precluded the ability to determine if they innervate the same PC (Spirou et al., 1990; Kuwabara et al., 1991; Rodríguez-Contreras et al., 2006). Kuwabara et al. noted that dual calyces are more frequently found in 1-2 week old animals and that these calyces were generally more spatially distinct than those in older animals, suggesting that loss of dual calyces may indicate a refinement of tonotopy (Kuwabara et al., 1991).

To address the outstanding question of competition in calyx development, the study detailed in chapter 2 was undertaken. This study utilizes serial block-face scanning electron microscopy (SBEM) to survey many cells in the medial portion of the MNTB at postnatal ages spanning P2P9. This process uses an ultramicrotome with diamond knife integrated within the electron microscope vacuum chamber capable of slicing ultrathin sections from the surface of a sample after it has been scanned. This results in excellent resolution in the image plane, minimal slice thickness, as well as a high degree of alignment between slices and low rate of loss of serial sections (Denk and Horstmann, 2004; Briggman and Bock, 2012). The dimensions of the resulting image volumes $(50-150 \mu m$ in each dimension $(x, y, z))$ are sufficient to examine convergence of calyceal axons in most cases. The results of this study should provide strong evidence of innervation states for PCs across calyx development and serve as a benchmark for further studies of calyx formation. 
A second and related question that remains outstanding in calyx development are the specific mechanisms inherent in the developing system that may dictate terminal competition. Several potential methods have already been discussed: decreasing PC resistance requiring stronger EPSCs to evoke an AP, synchronous pre- and postsynaptic firing to reduce NMDAR concentration and sharpen postsynaptic response, and regulation of PC gene expression through the BMP/SMAD pathway (Joshi et al., 2007; Hoffpauir et al., 2010; Xiao et al., 2013). Qualitative analysis has revealed another potential mediator of competition: polarity in somatic terminal placement; polarity in $\mathrm{CH}$ :MNTB development is the focus of the study in chapter 3.

\section{Polarity and Calyx Development}

Polarity is a hallmark characteristic of neurons, both in terms of structure and function. Morphological polarity and asymmetry in protein trafficking is a requirement for proper cell division, differentiation, migration, and function in all neurons. At the most basic level, neurons possess signal-receiving dendrites and signal-transmitting axons that define a polarized flow of information. In addition, gross neuronal polarity is reflected internally through positioning of intracellular structures such as the nucleus and centrosome; the relationship between orientation of these organelles and cellular polarity has persisted evolutionarily from unicellular life to the most complex living creatures (Bornens and Azimzadeh, 2007). Polarity is particularly vital to neuronal development. For example, polarized orientation of the mitotic division plane in cortical development determines whether a progenitor cell divides symmetrically to form additional progenitors or asymmetrically to produce neurons (Noctor et al., 2004). Mutations in the LIS1 protein (lissencephaly-related protein 1) - a dynein-interacting protein that is essential for aligning the mitotic division plane-leads to a disorder called lissencephaly characterized by a smooth brain surface devoid of gyri and sulci and multiple negative physical and mental phenotypes (Reiner et al., 1993; Pilz et al., 1998). Migration also requires coordinated and highly polarized structural changes in neurons, both in macrostructure and organelle 
rearrangement. A repetitive program of extension of a leading process, translocation of the centrosome into this process, and movement of the nucleus into the leading process allows cells to migrate and obtain correct placement within the developing brain (Higginbotham and Gleeson, 2007). The partitioning defective protein 6 (Par6), part of a polarity protein complex that also includes Par3 and atypical protein kinase $\mathrm{C}(\mathrm{aPKC})$, is involved in centrosomal protein aggregation, microtubule dynamics, centrosome movement, and nucleokinesis-all processes vital to neuronal migration (Etienne-Manneville and Hall, 2003; Hirano et al., 2005; Kodani and Tonthat, 2010). This complex is also necessary for proper axon specification; the polarity complex is highly localized to the axon growth cone in hippocampal neurons, and knockdown of any component results in loss of axon specification (Shi et al., 2003). More recently-and most importantly for our study_polarity proteins have been shown as key mediators of synaptogenesis. For instance, Lis1 heterozygous mice show decreased spine density, dendritic filopodial motility, and delayed synaptic cluster formation, suggesting Lis1 plays a role in synaptogenesis through its association and stabilization of the dynein motor complex (Sudarov et al., 2013). The planar cell polarity proteins Celsr3 (Cadherin EGF LAG seven-pass G-type receptor 3) and Vangl2 (Van Gogh-like protein 2) localize to glutamatergic synapses in the hippocampus and exert opposing effects; while loss of Celsr3 leads to a $50 \%$ reduction in hippocampal excitatory synapses, Vangl2 knockout actually increases synapse number (Thakar et al., 2017). Finally, there is evidence that Scribble1-another component of the planar cell polarity complex-recruits and localizes the Vangl1/2 protein through Par3, and that Vangl2 can bind the postsynaptic density protein PSD-95 (Yoshioka et al., 2013; Kharfallah et al., 2017).

In the MNTB, PCs are highly polarized, possessing eccentric nuclei that create a nucleus-poor "cytoplasmic pole" and nucleus-rich "nuclear pole" (Morest, 1968b; Smith et al., 1991; Hoffpauir et al., 2006; Rodríguez-Contreras et al., 2006). It has been observed previously that calyceal terminals appear to grow selectively on the "cytoplasmic pole" opposite the nucleus (Fig. 15B-D 
and 21C, Bollmann et al., 1998; Hoffpauir et al., 2006). If this somatic area represents postsynaptic specialization for supporting the growing calyx, a terminal occupying this space may have a distinct competitive advantage over terminals located closer to the nucleus. This phenomenon has not yet been studied either quantitatively or with respect to calyceal development. Applying SBEM, 3D reconstruction and morphological analysis, the study discussed in chapter 3 fills a portion of this gap in knowledge. 


\section{Chapter 2: Synaptic Inputs Compete During Rapid Formation of the Calyx of Held: A New Model System for Neural Development}

(reprinted with permission from the Journal of Neuroscience, see Appendix I)

Paul S. Holcomb ${ }^{1}$, Brian K. Hoffpauir ${ }^{1}$, Mitchell C. Hoyson ${ }^{1}$, Dakota R. Jackson ${ }^{1}$, Thomas J. Deerinck $^{4}$, Glenn S. Marrs ${ }^{1}$, Marlin Dehoff ${ }^{1}$, Jonathan Wu ${ }^{2}$, Mark H. Ellisman ${ }^{4,5}$, George A. Spirou ${ }^{1,2,3}$

${ }^{1}$ Center for Neuroscience, Departments of ${ }^{2}$ Otolaryngology, and ${ }^{3}$ Physiology and Pharmacology

West Virginia University School of Medicine Morgantown, WV 26506-9303, USA

${ }^{4}$ National Center for Microscopy and Imaging Research and ${ }^{5}$ Department of Neuroscience, University of California, San Diego, California 92092-0608, USA

Acknowledgements: Thanks to an additional 21 tracers who segmented neurons for 3D reconstruction and analysis, Brian Pope for tissue preparation, and Gerry Hobbs for assistance with statistical analysis. We appreciate the insightful comments of two anonymous reviewers. This work was supported by grants NIH R01 DC007695 to GAS, NIH F32 DC008730 to BKH, NIH F32 DC105462 to GSM, NIH/NIGMS CoBRE P30 GM103503 to the WVU Center for Neuroscience and NIH/NIGMS P41 GM103412 to the National Center for Microscopy and Imaging Research. 


\section{Abstract}

Hallmark features of neural circuit development include early exuberant innervation followed by competition and pruning to mature innervation topography. Several neural systems, including the neuromuscular junction and climbing fiber innervation of Purkinje cells, are models to study neural development in part because they establish a recognizable endpoint of mono-innervation of their targets and the presynaptic terminals are large and easily monitored. We demonstrate here that calyx of Held $(\mathrm{CH})$ innervation of its target, which forms a key element of auditory brainstem binaural circuitry, exhibits all of these characteristics. To investigate $\mathrm{CH}$ development, we made the first application of serial block-face scanning electron microscopy (SBEM) to neural development with fine temporal resolution and thereby accomplished the first time series for 3D ultrastructural analysis of neural circuit formation. This approach revealed a growth spurt of added apposed surface area (ASA) $>200 \mu \mathrm{m}^{2} /$ day centered on a single age at postnatal day $(P) 3$ in mice and an initial rapid phase of growth and competition that resolved to monoinnervation in $2 / 3$ of cells within three days. This rapid growth occurred in parallel with an increase in AP threshold, which may mediate selection of the strongest input as the winning competitor. ASAs of competing inputs were segregated on the cell body surface. These data suggest mechanisms to select "winning" inputs by regional reinforcement of postsynaptic membrane to mediate size and strength of competing synaptic inputs. 


\section{Introduction}

The overproduction of synaptic connections and subsequent pruning is a common theme in nervous system development (Purves and Lichtman, 1980). Model systems to study these events include the neuromuscular junction (NMJ; Wu et al., 2010), climbing fiber innervation of Purkinje cells (CF:PC; Watanabe and Kano, 2011 ) and retinal ganglion cell innervation of the dorsal lateral geniculate nucleus (RGC:dLGN; Hong and Chen, 2011) because nerve terminals are large, easily identified, and the end point of 1:1 or few:1 innervation ratio is easily quantified. In each of these systems, innervation begins as contact by multiple small inputs, proceeds through an early competitive stage whereby several inputs strengthen and completes by final pruning to, in most cases, a single "winning" input.

Another neural connection characterized by mono-innervation is the calyx of Held contact with principal cells of the medial nucleus of the trapezoid body $(\mathrm{CH}: \mathrm{MNTB})$, but there is uncertainty about the prevalence of competition among synaptic inputs (Hoffpauir et al., 2006; RodríguezContreras et al., 2008) and, therefore, whether it exhibits the hallmark developmental features of other neural systems (Borst and Soria van Hoeve, 2012). This typically mammalian neural connection is part of massive binaural convergence circuitry in the auditory brainstem (Warr, 1972). Light microscopic techniques established rapid growth of $\mathrm{CHs}$ in rats over several days during the first postnatal week (Kandler and Friauf, 1993; Rodríguez-Contreras et al., 2008). In mice, rapid growth in synaptic currents occurred in a narrow temporal window between P2-4. Both serial section electron microscopy (SSEM) and minimal stimulation physiological techniques in brain slices revealed only a small percentage of MNTB neurons innervated by multiple large inputs at P4 (2/19, 11\%; 4/29, 17\% respectively; Hoffpauir et al., 2006). Electrophysiological counting techniques revealed multiple small inputs onto most cells from late embryonic and younger postnatal ages (Rodríguez-Contreras et al., 2008; Hoffpauir et al., 
2010). Therefore, it seems plausible that the $\mathrm{CH}$ :MNTB also passes through hallmark developmental stages found in other neural systems.

To objectively assay the dynamics of calyx formation, we used an anatomical approach because physiological counting techniques provide an underestimate of convergent synaptic inputs due to sectioning of axons in brain slice preparations. Also, convergent inputs may share stimulation thresholds and be unresolvable. Serial block-face scanning electron microscopy (SBEM) provides high resolution to identify neuronal contacts and accurately registers cellular ultrastructure through large tissue volumes (Denk and Horstmann, 2004; Briggman et al., 2011). SBEM and related techniques were developed to generate maps of neural circuits, called connectomes, with the eventual objectives of comparing brains among normal and pathological states (Kleinfeld et al., 2011). Here we make the first application of these technologies to the developing brain with fine temporal resolution to reveal changing neural connections across the age range critical for calyx growth (P2-P9). We demonstrate early exuberant innervation, establish that competition is a common process and pinpoint $\mathrm{P} 3$ as a uniquely active day in $\mathrm{CH}$ growth during which cell apposition areas increase at a rate exceeding $200 \mu \mathrm{m}^{2} /$ day. These data provide metrics for $\mathrm{CH}$ growth dynamics more precise than for other model systems and exemplify utility of the $\mathrm{CH}: M N T B$ system for studies of synaptogenesis with relevance to other neural systems throughout the brain. 


\section{Materials and Methods}

\section{Ethical Approval}

All procedures involving animals were approved by the West Virginia University (WVU)

Institutional Animal Care and Use Committee and were in accordance with policies of the United States Public Health Service.

\section{Animals}

A breeding colony of mice (FVB/NJ; The Jackson Laboratory, Bar Harbor, ME, USA or FVB/NCr, National Cancer Institute, Frederick, MD, USA) was maintained within the vivarium at West Virginia University School of Medicine. Timed pregnancies were carried out by pairing mice overnight. Assuming fertilization occurred around midnight, gestation time was approximately 19 days (E0-E18) with birth typically occurring overnight. Animals were checked twice daily, so ages denoted herein are accurate to within $12 \mathrm{~h}$.

\section{Tissue Processing for Serial Block-face Electron Microscopy (SBEM)}

Unless otherwise indicated, all reagents were purchased from Sigma (St. Louis, MO). A single mouse of either sex was used at each age (P2, P3, P4, P6 and P9) with the exception of P2, where a second mouse was used to check data consistency. This second P2 mouse, as well as the P3 and P4 mice, were littermates; the mice used for P6 and P9 were littermates from a separate litter. Key image features were also compared and were consistent with those from separate animals in a different study where serial sections were imaged by transmission electron microscopy (Hoffpauir et al., 2006). Each mouse was anesthetized and perfused with normal Ringer's solution containing xylocaine $(0.2 \mathrm{mg} / \mathrm{ml})$ and heparin $(20$ units $/ \mathrm{ml})$ for 2 minutes at $35^{\circ} \mathrm{C}$ followed by $0.15 \mathrm{M}$ cacodylate buffer containing $2.5 \%$ glutaraldehyde (Polysciences, Inc., Warrington, PA), 2\% formaldehyde (Fisher Scientific, Waltham, MA) with 2 mM calcium chloride at $35^{\circ} \mathrm{C}$ for 5 minutes. Brains were removed from the skull and postfixed for an additional 18 hours on ice in the same solution. Brain tissue was cut into $200 \mu \mathrm{m}$ thick sections 
in the coronal plane using a vibratome (Ted Pella, Inc., Redding, CA) in ice-cold 0.15M cacodylate buffer containing $2 \mathrm{mM}$ calcium chloride then washed for 30 minutes in the same solution. The MNTB was identified in free-floating sections using a dissecting microscope and the superior olivary complex containing the MNTB, with surrounding tissue $(\sim 1.5 \times 1.5 \mathrm{~mm})$, was dissected from the tissue. The tissue was photographed and the location of the MNTB was mapped relative to contours of the brain surface and cut edges of the tissue block. A solution containing 3\% potassium ferrocyanide (Electron Microscopy Sciences, Hatfield, PA) in 0.3M cacodylate buffer with $4 \mathrm{mM}$ calcium chloride was combined with an equal volume of $4 \%$ aqueous osmium tetroxide and added immediately to the tissue for $1 \mathrm{hr}$ at room temperature (RT). Tissue was processed sequentially through filtered $1 \%$ thiocarbohydrazide (Electron Microscopy Sciences; all water was nanopure filtered; Nanopure Diamond, Barnstead International, Dubuque, lowa)) for 20 minutes at RT, $2 \%$ osmium for 30 minutes at RT and $1 \%$ uranyl acetate overnight at $4^{\circ} \mathrm{C}$ following triple rinses in $\mathrm{H}_{2} \mathrm{O}$ for 5 minutes each between each step. Tissue was triple rinsed in $\mathrm{H}_{2} \mathrm{O}$ at $\mathrm{RT}$ for 30 minutes. A lead aspartate solution was prepared (0.066 gm of lead nitrate, (Electron Microscopy Sciences), dissolved in $10 \mathrm{ml} 0.003 \mathrm{M}$ aspartic acid solution, $\mathrm{pH}$ adjusted to 5.5 with $1 \mathrm{~N} \mathrm{KOH}$, warmed in a $60^{\circ} \mathrm{C}$ oven for 30 minutes) and filtered. Sections were placed into filtered lead aspartate solution in the $60^{\circ} \mathrm{C}$ oven for 30 minutes. The tissue was rinsed 5 times for 3 minutes in $\mathrm{H}_{2} \mathrm{O}$ and then dehydrated through graded alcohols into acetone and flat-embedded in Durcopan resin (Electron Microscopy Sciences) between mylar strips and placed in a $60^{\circ} \mathrm{C}$ oven for 48 hours. Tissue samples were photographed and shipped to the National Center for Microscopy and Imaging Research (NCMIR, University of California San Diego) for imaging. The MNTB was identified by comparing photographs of tissue before and after staining. 


\section{SBEM Imaging}

Resin-embedded tissues were mounted on aluminum specimen pins (Gatan, Inc., Pleasanton, CA) using cyanoacrylic glue and precision trimmed with a glass knife to a rectangle approximately $0.5 \mathrm{~mm} \times 0.75 \mathrm{~mm}$ so that tissue was exposed on all four sides. Silver paint (Ted Pella Inc., Redding, CA) was used to electrically ground the edges of the tissue block to the aluminum pin. The entire specimen was then sputter coated with a thin layer of gold/palladium to enhance conductivity. After the block was faced with a 3View ultramicrotome unit (Gatan) to remove the top layer of gold/palladium, the tissue morphology became visible by back-scattered electron (BSE) detector imaging and the remaining coating on the edges of the block served to reduce charging. A low magnification image ( 500X) was collected to identify the MNTB and midline and target the medial MNTB for serial image collection. Tissue blocks were scanned using an FEI Quanta FEG SEM (FEI, Hillsboro, OR, USA) or Zeiss Merlin scanning electron microscope (Car Zeiss, Inc., Thornwood, NY) and sectioned at a thickness of $60 \mathrm{~nm}$ or $40 \mathrm{~nm}$ (P9). Accuracy of section thickness was estimated by assuming circularity of mitochondria and comparing the diameter of longitudinally oriented organelles with diameters measured in the image plane (Wilke et al., 2013). These values approximated the nominal section thickness (P2 58.4 nm, P3 55.3 nm, P4 56.5 nm, P6 53.2 nm, P9 35.9 nm). Tissue dimensions and pixel sizes are indicated in Table 1.

\section{Segmentation and Three-dimensional Reconstruction}

Image files were shipped to WVU for analysis. Cells were segmented, reconstructed and analyzed using the IMOD 3D reconstruction and visualization software (Boulder Laboratory for 3-D Electron Microscopy of Cells, University of Colorado, Boulder). Most reconstructions were completed using either a Velocity D55SQ workstation (Xeon X5677 3.46GHz quad core processor, 96 GB RAM, 640GB SSD storage, 2.4TB 15k RPM SAS storage, Linux Red Hat Enterprise Release 5.7; Pogo Linux Inc., Redmond, WA), a Dell Precision T7500 desktop 
computer (Dual Intel Xeon X5687 3.6GHz quad-core CPUs, 48GB RAM, 240GB SSD storage, 2.5Tb 15k RPM SAS storage, Windows 7 Professional 64-bit OS; Dell, Inc., Round Rock, TX), or a Dell Precision T1500 desktop computer (Intel Core i5-750 2.67GHz quad core processor, 16GB RAM, 600GB SSD, Windows 7 Professional 64-bit OS; Dell, Inc., Round Rock, TX). Each computer was interfaced to an interactive display drawing tablet and pen (Model DTU-2231, Wacom, Otone, Japan) for manual segmentation of objects within the tissue volume. Many reconstructions were performed on image volume subsets on laptop computers running IMOD and interfaced to a Wacom Bamboo Splash drawing tablet. Neurons were identified within each SBEM data set and differentiated from glia by ultrastructural criteria (Peters et al., 1991). Cells were omitted from tracing if a portion of the cell body was truncated by the bounds of the data set, or if a sufficient portion of an input extended outside of the data volume to prevent its identification as a distinct input. The nucleus, cell body and all neural inputs were segmented for 58 cells across tissue samples from P2-P6 and dendrites were segmented for 51 of these cells. Reduced quantitative analysis of only input number and size was assessed for an additional 61 cells across this age range; inputs were tracked to separate axons and axons were tracked through the image volume. Qualitative determination of a dominant large input was possible for 57 of the 66 cells present in the P9 volume due to the large size of the terminal. For cells at P9 where a dominant large input was not evident (9/66), quantitative analysis of ASA was performed. A large tracing team (total 28 people) completed the segmentations. We established a ranking process of expert and junior level competency for tracing team members to minimize the two most common types of errors in segmentation: (1) cellular features were missed because parts of cellular objects (e.g. cell body, neural input) were overlooked or mistakenly considered not to be connected to an object, or (2) new features were introduced by fusing two different objects resulting from uncertainty in aligning small caliber structures $(<100 \mathrm{~nm})$ across sections. Expert tracers (initially GS and BH who completed a previous serial section EM study of the MNTB, Hoffpauir et al., 2006) were identified by their experience and absence of errors 
during segmentation. Errors in category 1 were addressed by having expert tracers proof all of the work of junior tracers. Category 2 issues were identified during tracing and were resolved by review with an expert tracer and, if necessary, tracking the questioned object through the image volume to determine if it was a part of a different cell. All junior tracers were instructed to highlight uncertainties in tracking objects across sections for resolution by expert tracers. A final check was to examine rendered structures for atypical features, such as obtuse branching angles or sudden changes in dimension, and re-inspect these structures in the image volume. Consistent absence of these defined errors led to promotion of junior tracers to expert status. To balance efficiency and accuracy, manual segmentation was performed on every third section containing each object; comparisons between traces of every section and every third section yielded a difference of $<5 \%$ in total surface area. Apposed surface areas were traced at the contact between each presynaptic input and its postsynaptic partner and meshed using the IMOD meshing tool, creating a two-dimensional sheet-like surface. Surface area calculations were accomplished using the imodinfo command, which calculates the total surface area by summing all of the triangular areas composing the meshed surface (http://bio3d.colorado.edu/imod/doc/man/imodinfo.html). All binning and ratio calculations were performed using Microsoft Excel (Microsoft, Redmond, WA) and graphs were generated using MATLAB (The MathWorks, Inc., Natick, MA).

\section{Electroporation and Imaging of Acute Brain Slices}

Mouse whole-head or brainstem slices $(250-350 \mu \mathrm{m})$ were generated using a Compresstome VF-200 tissue cutter (Precisionary Instruments, Greenville, NC, USA) in ice cold oxygenated artificial cerebrospinal fluid (ACSF). Extracellular solution of ACSF consisted of (in mM): 125 $\mathrm{NaCl}_{2}, 2.5 \mathrm{KCl}, 1 \mathrm{MgCl}_{2}, 2 \mathrm{CaCl}_{2}, 25$ glucose, $25 \mathrm{NaHCO}_{3}, 1.25 \mathrm{NaH}_{2} \mathrm{PO}_{4}, 0.4$ ascorbic acid, 3 myo-inositol, and 2 Na-pyruvate, at a pH of 7.3. A total of 8 mice aged P3 (7 animals) -P4 were used to generate brainstem tissue slices for these experiments. Following sectioning, slices 
were allowed to cool to room temperature before transfer to an in vitro recording chamber, where they were maintained in oxygenated ACSF at $35^{\circ} \mathrm{C}$ (dual in-line heating units, and a heated stage with an RC-27L tissue chamber and TC-344B temperature control module; Warner Instruments, Hamden, CT) until completion of electroporation labeling (typically less than $2 \mathrm{hrs})$. Constant perfusion of oxygenated ACSF ( $2 \mathrm{ml} / \mathrm{min})$ was supplied by a Valvelink 8 perfusion system (Automate Scientific, San Francisco, CA). All data collection for imaging or electrophysiological experiments was complete within $6 \mathrm{~h}$ of tissue sectioning.

Focal application of fluorescent markers was accomplished by selective targeting of afferent fiber bundles at the ventral midline and proximal to the MNTB. Afferent fibers and terminals were labeled by electroporation of Alexa fluorescent dyes ( $5 \%$ in phosphate buffered saline (PBS)) coupled to dextran (Alexa 488 or 548; Invitrogen, Carlsbad, CA). Electroporation pulses of 20-40V were delivered in sets of 10 pulses at $16 \mathrm{~Hz}$ through pipettes of 2-3 $\mathrm{M} \Omega$ resistance. Stimulation protocols were created and controlled by pClamp 9.2 software (Molecular Devices, Sunnyvale, CA) and administered through an S-940/S-910 stimulus isolation system (Dagan, Minneapolis, MN). Pipette guidance and differential interference contrast (DIC) observation of tissue slices was performed with an Axioskop II plus microscope (Zeiss, Thornwood, NY) with a 40X (0.75 NA) dipping lens.

Image stacks (Z-step size 0.7-1.2 $\mu \mathrm{m}$ ) were collected using either a Zeiss LSM510 confocal microscope or custom-built two photon (2P) microscope (Yang et al., 2013). Confocal microscopy was performed using a 20X (0.75 NA) PLAN NEOFLOUR lens. The 2P system was comprised of a Verdi-V18 pumped, Mira-HP Ti-Sapphire laser (Coherent Inc., Santa Clara, CA) mode-locked at $780 \mathrm{~nm}$, an upright microscope (Moveable Microscope, Sutter Instrument, Novato, CA) equipped with a scanner (Cambridge Instruments, Somerville, MA) and 20X (1.0 NA) PLAN APO dipping lens (Olympus, Center Valley, PA). Image acquisition was controlled using Scanlmage software (Pologruto et al., 2003) operating on a MATLAB platform. Images 
were converted to tiff format and imported into Imaris (Bitplane, South Windsor, CT) for viewing and qualitative analysis. Images of the compressed image stacks were imported into Photoshop (Adobe, San Jose, CA) for highlighting and figure creation.

\section{Calcium Imaging and Electrophysiology}

Data are re-plotted and re-analyzed from Hoffpauir et al. (Hoffpauir et al., 2010) and that manuscript should be consulted for detailed experimental methods. Briefly, for minimal stimulation experiments a dual shaft tungsten microelectrode (FHC, Bowdoinham, ME) was positioned over trapezoid body afferent fibers at the ventral midline. Tissue visualization for these experiments was performed on the Axioskop II system with constant ACSF perfusion at $35^{\circ} \mathrm{C}$ as described above. Connected cells were identified by evoked calcium signals following loading with Fura-2-AM (Invitrogen, Grand Island, NY). Excitation light at $340 \mathrm{~nm}$ and $380 \mathrm{~nm}$ was supplied by rapid filter switching with a Lambda DG-4 system (Sutter Instrument) controlled by $\mathrm{HC}$ Image software (Hamamatsu, Sewickley, PA) and images were captured at $25 \mathrm{~Hz}$ with a Hamamatsu C9100-12 EM-CCD camera. Responsive cells were targeted for whole-cell voltageclamp recording $\left(\mathrm{V}_{\text {hold }}=-70 \mathrm{mV}\right)$ using a multiclamp 700A microelectrode amplifier, Digidata 1322A digitizer, and CV-7A headstage controlled by pClamp 9.2 software (Molecular Devices). Data were acquired at a sampling rate of $50 \mathrm{kHz}$ and low-pass filtered at $6 \mathrm{kHz}$. Series resistance compensation was set to at least $70 \%$ for all recordings. Internal recording solution for voltage clamp contained (in mM): $114 \mathrm{~K}$-gluconate, $26 \mathrm{KCl}, 2 \mathrm{MgCl}_{2}, 0.1 \mathrm{CaCl}_{2}, 1.1 \mathrm{EGTA}$ $\mathrm{Na}_{4}, 10$ HEPES, 5 Na-phosphocreatine, and 4 ATP-Mg, at $\mathrm{pH}$ 7.3. Trapezoid body stimuli were varied in $2 \mathrm{~V}$ increments from $2-40 \mathrm{~V}$. A selected minimal stimulation set was repeated 4 times in a randomized presentation order to circumvent effects of stimulus order. 


\section{Statistical Analysis and Curve-fitting}

The increase in average ASA size between P2-6 was assessed using non-parametric tests, since size distributions were skewed toward large values and had increasing variability with age. For these reasons, data were transformed so that standard deviations were identical and the hypothesis that means increased each day from P2-4 was tested. A Jonckheere-Terpstra Test was used to assess overall significance and a post hoc test attributed to Hayter-Stone in Hollander and Wolf (1999) was used to assess differences between individual pairs of means. Other statistical analysis utilized the t-test. Values reported in the text are mean \pm standard deviation.

The rate for emergence of dominant, large inputs was determined using the following equation:

$$
y=A\left(1-e^{\frac{-(t-2)}{\tau}}\right)
$$

Where $t$ is age, $\mathrm{T}$ is the time constant, $\mathrm{A}(=100)$ normalizes the maximum (asymptotic) value to $100 \%$, and (t-2) shifts the beginning value to $\mathrm{P} 2$.

Data for calyx growth were fit to a Boltzmann equation and compared to fits for maturation of AP firing phenotype and AP threshold to current injection (Hoffpauir et al., 2010):

$$
Y=\frac{A_{1}-A_{2}}{1+e^{\frac{t-t_{1 / 2}}{k}}}+A_{2}
$$

Where $A_{1}$ and $A_{2}$ are the initial and final conditions, respectively, $t$ is age, $t_{0.5}$ is the age of halfmaturation, and $\mathrm{k}$ is the slope factor. 


\section{$\underline{\text { Results }}$}

\section{Serial block-face electron microscopy (SBEM) is a powerful tool to investigate nervous system development}

In order to quantify growth dynamics of the calyx of Held, resolution of cellular ultrastructure was necessary to assay the size and extent of physical contact between nerve terminals and the postsynaptic neuron, verify that terminals contained structural elements of synapses and exclude frequent axon-somatic contacts in the developing MNTB that lack synaptic machinery (Hoffpauir et al., 2006). Furthermore, although most cells of the MNTB in mice are considered principal cells, a small percentage of neurons may be of a different cell type, and not innervated by calyces of Held (Banks and Smith, 1992). We considered that ultrastructural criteria might be useful to identify non-principal cells at young postnatal ages and exclude them for a more accurate analysis of calyx growth dynamics.

Tissue blocks were collected at multiple postnatal time points (P2-4, P6, P9; Fig. 22A), encompassing salient changes in mouse MNTB innervation (e.g., increases in terminal size, strength of evoked EPSCs, and innervation by multiple inputs) as described in previous studies (Kandler and Friauf, 1993; Hoffpauir et al., 2006, 2010; Rodríguez-Contreras et al., 2008). Minimal stimulation electrophysiological techniques in rat indicated that nearly all MNTB cells (93\%) are mono-innervated by P9 (Bergsman et al., 2004). This dense temporal sampling provided a time series view of neural development, albeit using a different animal at each age, at ultrastructural resolution. We addressed fundamental questions about $\mathrm{CH}$ :MNTB development such as the number of small inputs preceding calyx growth, the extent of competition during calyx growth, the speed of calyx growth and the age by which all MNTB cells were contacted by large inputs (question marks in Fig. 22A). High-resolution image stacks, oriented in the coronal plane at all ages, were obtained from the medial portion of the MNTB using SBEM (Fig. 22B), providing a robust and highly detailed data set for each sampled age. 
The medial MNTB was sampled to avoid potential regional differences in maturation within this cell group (Rodríguez-Contreras et al., 2008; Ford et al., 2009). Sampled volumes ranged from approximately 400,000 to $1,000,000 \mu \mathrm{m}^{3}$ (Table 1 ). This age range and specified sample region permitted a systematic and dynamic view of calyx and principal cell development amenable to quantitative analysis.

MNTB principal neurons and their synaptic inputs were manually segmented from the image volume. The nucleus, cell body, dendrites and axon were segmented as distinct objects, as was each somatic input and its associated axon (Fig. 22C). This approach yielded high-resolution three-dimensional representations of neurons and their synaptic inputs across the age range selected for analysis. A representative section through a cell with two large inputs (largest input (1) colored blue; second largest input (2) colored green) is depicted in Fig. 22C. We noted that glial processes could intervene between a neural input and the principal neuron and reduce the contact area between cells (labeled g, purple structures and open purple arrowheads, respectively, in Fig. 22C). Therefore, the contact between input and somatic membrane was segmented separately and reconstructed for each input to yield the apposed surface area (ASA). The ASA is representative of the available space for both current and potential synaptic contacts to form between the growing terminal and the MNTB cell body. ASA size can be measured accurately in these images and was used as the metric for synaptic strength, especially given uncertainty that all postsynaptic densities were resolved. In addition, synaptic strength may not scale linearly with size or density of synaptic structures due to variance in numbers of docked vesicles, vesicle pool size and density of glutamate receptors. All large terminals (see below) were filled with synaptic vesicles and multiple synapses could be identified within each terminal, consistent with our earlier report using higher resolution imaging over smaller serial section volumes (Hoffpauir et al., 2006). Functional inputs were identified, then, as having synaptic vesicles clustered at membrane apposition sites (sv, Fig. 22C). In a 
subset of terminals at P6 $(n=7)$, where smaller pixel dimensions (Table 1) rendered identification of the active zone $(A Z)$ more reliable, $A Z$ size was linearly related to $A S A\left(R^{2}=\right.$ 0.97). The ASA, then, was used to distinguish innervation territories of multiple inputs, investigate the prevalence of synaptic competition, and quantify the dynamics of calyx growth.

\section{P2: MNTB cells are contacted by multiple small inputs}

At P2, as has been shown previously in a small tissue sample (Hoffpauir et al., 2006), neurons in the MNTB are packed tightly (cells colored gold and numbered in Fig. 23A) with large areas of cell-cell contact (white arrowheads between cells 1 and 2, 3 and 4). Even in single sections, neurons appear to be extensively covered by glial processes (purple structures in Fig. 23A). Consequently, a large portion of the cell membrane is inaccessible for innervation. Somatic neural inputs, identified by collections of synaptic vesicles in regions of directly apposed preand postsynaptic membrane (Fig. 23A, inset), were small (average ASA of $2.38 \pm 2.5 \mu m^{2} ; n=$ 21 cells, 204 inputs total; Fig. 23B). The size distribution revealed many small inputs with few inputs having an ASA > 5 $\mu \mathrm{m}^{2}$ (25 of 204 inputs, 12.3\%; Fig. 23B). The inputs to each cell are plotted in order of increasing size and linked by lines in Fig. 23C. Most cells had between 5-12 inputs on the cell body (average $9.3 \pm 6$ inputs; Fig. 23C). Three-dimensional reconstructions depict the principal cells of the MNTB at the earliest stage of input formation, with multiple small inputs impinging upon each cell. Most somatic inputs at P2 were en passant in morphology (most inputs in Fig. 23D, E), even though they occurred in branches of axons that passed near the cell body (e.g. inputs 1 and 8 in Fig. 23D). These latter axons had periodic swellings that were filled with synaptic vesicles and which frequently made synaptic contacts with dendrites of neighboring MNTB principal cells (arrowheads in Fig. 23D show example regions of axon filled with vesicles). One of the largest inputs at this age, termed a protocalyx by Morest (1968), (black arrowhead in Fig. 23E) may have been captured in the earliest stage of expansion over the somatic surface. 
We considered that some inputs destined to form calyces may initially contact the dendrites of MNTB cells, as occurs for auditory nerve fiber innervation of bushy cells in the ventral cochlear nucleus (Mattox et al., 1982), and that round vesicle shape is not as reliable an indicator in neonatal animals of excitatory input as in adults (Vaughn, 1989). Since large diameter fibers of the trapezoid body originate from globular bushy cells of the ventral cochlear nucleus and give rise to calyces in the mature brain (Warr, 1972; Spirou et al., 1990; Smith et al., 1991), we identified large diameter fibers at P2 (average $1.59 \pm 0.4 \mu \mathrm{m}$ diameter) and mapped several of these fibers and their branches (example shown in Fig. 24A). We discovered that large diameter axons branched 2-4 times in the image volume, and these branches further branched so extensively that a single fiber may contact over twenty MNTB cells $(22.5 \pm 12, n=12$; center of contacted MNTB cells plotted as green spheres in Fig. 24A). Conversely, we investigated whether multiple large diameter axons had branches that converged via somatic and dendritic inputs onto a single MNTB cell (Fig. 24B). We identified cells contacted by 4 or more of the 12 large diameter axons that were reconstructed. The cell in Fig. 24B was contacted on its soma by 4 of the 5 large diameter axons depicted; ASAs for 3 of these 4 somatic inputs are shown in the inset. Of the 71 identifiable MNTB cells in this image volume (because their cell bodies were entirely contained within the volume), $36(51 \%)$ were contacted by at least 4 of the 12 reconstructed axons. By extrapolating from these values, 24 large diameter axons $(2 \times 12)$ would generate at least 4 inputs onto all 71 cells, which is fewer than the estimated 60 axons that will mono-innervate these cells in maturity (assuming 18\% of globular bushy cells generate 2 calyces as in rat; Rodríguez-Contreras et al., 2006). It is likely that axons eventually innervating MNTB cells outside of this population of 71 cells also transiently innervate the cells included in the P2 volume, so we suggest that convergence from at least 4 separate globular bushy cells onto each MNTB cell is a common occurrence. Large diameter axons very rarely, if ever, branch medially to the MNTB into two large diameter axons in rodents (Friauf and 
Ostwald, 1988; Kandler and Friauf, 1993; Rodríguez-Contreras et al., 2006; Hoffpauir et al., 2010; see below), so these axons represent independent inputs.

\section{P3: Initiation of calyx growth}

Principal neurons at P3 displayed less cell-cell contact, allowing for greater contact with nerve terminals than was possible at P2 ( 3 cells indicated in Fig. $25 \mathrm{~A}$ ) but still have a substantial investment of glial covering (purple structures contacting cell bodies in Fig. 25A). Many inputs were larger than at P2 and generated a much broader distribution and larger average value of ASA $\left(10.7 \pm 35 \mu \mathrm{m}^{2}, \mathrm{n}=37\right.$ cells, 298 total inputs; Fig. 25B). The histogram of ASA values was multimodal, reflecting populations of small and larger inputs. The first minimum inflection point of this histogram occurred at $25 \mu^{2}$ (vertical dashed line in Fig. 25B), which was defined operationally as the threshold for differentiating small inputs from large inputs (Fig. 25B, red and black histogram bars, respectively). The inputs to each cell are plotted in Fig. 25C in order of increasing size and linked by lines, as in Fig. 25C. Most inputs (92\%) at P3 were small and most cells were contacted only by small inputs $(21 / 37$ (57\%) cells, red symbols in bottom graph of Fig. 4C; example cell shown in Fig. 25D). Nearly one-half of cells (16/37, 43\%) had at least one large input (open and closed black symbols in upper graph of Fig. 25C). Example cells are shown in Fig. 25E-F'; note that in 3D reconstructions the largest and second-largest inputs are colored blue and green, respectively, independent of the number of large inputs. Of these 16 cells, $8(50 \%)$ had at least two large inputs indicative of competition among growing calyces. One of these cells had 3 large inputs (asterisk in Fig. 25C). Considering that some cells with a single large input (Fig. 25E) may have already proceeded through a phase of competition, we considered $50 \%$ to be a minimum estimate of the fraction of MNTB neurons competed for by multiple large terminals with the potential to form calyces (termed calycigenic). Rotated views of a single MNTB neuron illustrate two large inputs (Fig. 25F, F'). 
We performed a more refined analysis of competition in attempt to distinguish cells in which one large input has become the dominant input. For cells having at least one large input, we calculated the ratio of the two largest inputs onto each cell. A ratio near 1 indicates inputs of similar size and suggests an active competitive process. The distribution of these ratios showed a grouping of cells ( 9 of 15 cells, $60 \%$ ) with an ASA ratio of 5 or less. Based upon this metric, we defined this group of cells to be contacted by actively competing inputs (open histograms in Fig. 25B'; same cells plotted as open diamonds in Fig. 25C). We then defined ASA ratios greater than 5 (6 of 15 cells, $40 \%$ ) to indicate a dominant large input (solid black histogram and circles in Fig. 25B', C, respectively) and considered the inputs to these cells not to be undergoing active competition. This quantification distinguished three categories of MNTB neurons: (1) those with only small inputs likely poised to begin growth, (2) those with actively competing inputs, (3) those with a dominant large input. Cells in groups 1 or 2 tended to have a greater number of inputs than those in group 3 (8.7 \pm 4 inputs for groups 1 and 2 vs $5.7 \pm 3$ inputs for group 3, p <0.00001, Student's t-test on pooled data P2-P6). This situation may be a consequence of regression of some small inputs as another subset of small inputs become large.

3D reconstructions demonstrate that these three groups of cells reflect different stages of calyx growth. Axons that gave rise to small inputs onto MNTB cell somata often had an en passant morphology and larger axonal swellings nearby that were filled with synaptic vesicles (white arrowheads in Fig. 25D), as at P2. Small inputs could branch from large terminals onto adjacent cells (input 5, Fig. 25D), can be functional (Rodríguez-Contreras et al., 2008) and may persist into adulthood (Kuwabara et al., 1991). The largest calycigenic input, whether defined as actively competing or a dominant input, typically enlarged from the base of the axon over the somatic surface with sinuous branches radiating into the extracellular space (Fig. 25E, F, F', blue and green). Note that for one of the MNTB cells with competing inputs, both inputs were 
similar in size and among the largest of this group (symbols enclosed by ellipsoid and labeled F in Fig. 25C, 3D views in Fig. 25F, F'). The largest inputs $\left(>200 \mu \mathrm{m}^{2}\right)$, however, were from the dominant large input group (closed black circles), suggesting that competition is resolved prior to the final stages of calyx growth (Fig. 25C).

\section{P4: Rapid calyx growth onto all postsynaptic cells}

Principal cells continue to enlarge and maintain their separation from one another between P3 and P4 (single P4 cell shown in Fig. 21C). Analysis of input size and distribution was performed for P4 as at earlier ages. The first minimum inflection point in the histogram of input sizes occurred at $25 \mu \mathrm{m}^{2}$ (Fig. 26A, dashed line), which defined, as at P3, the distinction between small (red) and large (black) terminals. Rapid growth of calycigenic terminals over this 24-hour period led to a large increase of average ASA to $60.6 \pm 108 \mu \mathrm{m}^{2}$ ( $n=18$ cells, 83 total inputs). All cells at P4 were contacted by at least one large input and most cells (16 of 18) had at least one input $>100 \mu \mathrm{m}^{2}$ in size. The histogram of ASA ratios revealed a break at a value of five, as at P3, to distinguish cells with actively competing from those with a dominant large input. There was an increase over P3 in the fraction of cells having a dominant large input (12 of 18 cells, $67 \%$ vs $40 \%$ at P3; Fig. $\left.26 A^{\prime}\right)$. This change in innervation profile suggests that a subset of small inputs at P3 grew quickly and competing inputs detected at P3 likely resolved their competition quickly within the 24-hour developmental period between P3 and P4. Several features of innervation were similar to P3. Most cells had one or two large inputs, but two cells had three large inputs (labeled $\mathrm{C}$ and $\mathrm{F}$ in Fig. 25B; 3D reconstructions shown in panels $\mathrm{C}$ and $\mathrm{F}$ ). On one cell (labeled F in Fig. 25B) these three inputs were considered to be in active competition. A single cell could have both competing inputs among the largest of all competing inputs (symbols shaded by blue ellipsoid in Fig. 26B and pictured in Fig. 26E). The largest inputs $\left(>260 \mu \mathrm{m}^{2}\right)$ were the dominant inputs on their respective cells (Fig. 26B-D). The presence of some cells at P3 without large inputs and all cells at P4 with large inputs but a wide range of sizes (Fig. 26B) 
supports the idea that initiation of calyx formation within a specified region of the MNTB is relatively well-synchronized within approximately a 24-hour period.

\section{P6: Increased numbers of mono-innervated cells}

At P6, a larger volume of the medial portion of the MNTB was captured than at P3 yet it contained a similar number of cells (43 cells), due in part to their wider spacing (Fig. 27A). The input size histogram revealed a minimum inflection point of $35 \mu \mathrm{m}^{2}$, similar to $\mathrm{P} 3$ and $\mathrm{P} 4$, to distinguish small from large inputs (Fig. 27B). Similar to P4, all but one cell at P6 (42/43) had at least one large input. Some cells had more small inputs than at P4, so the average ASA decreased to $42.6 \pm 105 \mu \mathrm{m}^{2}$ ( $\mathrm{n}=43$ cells, 306 total inputs). The histogram of input size ratios was divided into values greater or less than five, as at P3 and P4, defining cells with actively competing inputs $(<5)$ or a dominant large input $(>5)$ (Fig. 27B'). The fraction of cells with a dominant large input increased slightly to $70 \%$ (32/43) from $67 \%$ at P4. Many cells at P6 were contacted by a clearly defined immature calyx (> $250 \mu \mathrm{m}^{2}, 19$ of 43 cells, $44 \%$ ), which spread over much of the MNTB cell body (3D rendering in Fig. 27D, labeled D in Fig. 27C). Of the cells with large inputs, only 2 cells had three large inputs (asterisks in Fig. 27C) and these had size ratios $<5$ indicative of active competition. A single cell could have both competing inputs among the largest of all competing inputs as at P3 and P4 (symbols shaded by blue ellipsoid in Fig. $27 \mathrm{C}$ and pictured in Fig. 27E). Also like P3 and P4, the largest inputs were dominant inputs (Fig. 27C). Six MNTB cells with actively competing inputs did not have any input $>100 \mu \mathrm{m}^{2}$ and one MNTB cell had only small inputs. These cells, and one additional cell that was classified as nonprincipal due to its small size, deeply invaginated nucleus and limited cytoplasmic volume (not shown), were located among the most medial members of the cell group. 


\section{P9: Mono-innervation Established on Nearly All Principal Cells}

Since not all MNTB cells at P6 had a dominant large input, we analyzed an image volume from P9. This image volume was larger than at earlier ages (Table 1) and contained 66 neurons. Most cells (58/66) had a dominant large input that could be identified qualitatively. A small percentage of cells (8/66) appeared to have multiple smaller inputs. The ASAs for these inputs were quantified, indicating that $7 / 8$ cells had at least one input defined as a large input $(>25$ $\mu \mathrm{m}^{2}$ ), but all of these large inputs were $<100 \mu \mathrm{m}^{2}$. The input size ratio for these 7 cells was $<5$, indicating that a dominant input had not been resolved. The cells without a dominant large input clustered at the medial edge of the MNTB, but appeared similar morphologically to other MNTB principal cells. Thus, competition had resolved on all MNTB neurons except those contained in this cell cluster.

\section{Tracing large inputs to their axons of origin}

A subpopulation of globular bushy cells branch distant from their terminals and give rise to two calyces (instead of one), each onto nearby MNTB cells (cat, Spirou et al., 1990; Smith et al., 1991; rodent and bat, Kuwabara et al., 1991; estimated 18\% in rat, Rodríguez-Contreras et al., 2006). In rare instances in rat, a single axon could branch near its target (as close as $15 \mu \mathrm{m}$ ) and appear to deliver two large terminals onto the same cell (Rodríguez-Contreras et al., 2006). Since we studied the medial MNTB, many axons were traceable within the SBEM volume to distinct parent axons in the trapezoid body fiber fascicles. Branching of inputs near their targets

was captured except for cells at the borders of our image volume. Although in rodents branching of trapezoid body axons outside of the MNTB occurs rarely if at all (Friauf and Ostwald, 1988; Kuwabara et al., 1991; Rodríguez-Contreras et al., 2006), we considered that some competing inputs onto MNTB cells might originate from the same parent axon due to branching outside of the image volume. To address this issue, we electroporated small numbers of axons in mouse brain slices at P3 and P4 with Alexa dyes (Rodríguez-Contreras et al., 2008; Hoffpauir et al., 
2010) and imaged their trajectories within and emerging from fiber bundles in the trapezoid body using confocal or 2-photon microscopy (Fig. 28). Axons diverged separately from each small electroporation site and were distinguishable in the trapezoid body (not shown) prior to entering the fields of view depicted in this figure. Our goal was to label a small enough number of axons in each preparation to evaluate whether branches of single axons converged into the same vicinity via large nerve endings. Only $1 / 20$ axons studied in this fashion exhibited the potential for such convergence (axon highlighted in yellow and putative terminals by white arrows in Fig. $28 \mathrm{~A})$. For this axon, the nerve terminals were approximately $5 \mu \mathrm{m}$ apart, but it was difficult to assess whether they contacted the same cell. An angled view from the image stack suggested that the inputs contacted different postsynaptic structures due to curvature of the larger terminal (asterisks Fig. 28A, inset). An example of more typical branching patterns and convergence are shown in Fig. 28B. One axon is highlighted in yellow and expands into a single large terminal. Another labeled axon also ends in a large terminal, possibly onto the same but more likely onto a neighboring cell body (labeled "2" in Fig. 28B). A third axon (labeled "3" in Fig. 28B) delivers a

large terminal that in turn extends long, thin processes. These data confirm that the incidence of single axons that branch at distant locations and converge onto single MNTB cells via large terminals is very rare and that our estimates of synaptic competition reflect true convergence of neuronal projections.

\section{Dynamics of calyx growth and synchronization with postsynaptic maturation}

Emergence of a dominant, large input. Figure 29A shows a histogram summary of the distribution of MNTB cells across age based upon categorization of their inputs: (1) cells with only small inputs (red), (2) cells with actively competing inputs (open black, ASA ratio < 5), and (3) cells with a dominant large input (solid black, ASA ratio > 5). The growth of large inputs onto MNTB cells is a rapid and well-synchronized process, as indicated by the elimination (with one exception at P6 and P9) of cells with only small inputs between P2 and P4, and a significant 
increase in the percentage of cells with a dominant, large input from P3 to P4. These data also permitted quantification of the rate at which a single input emerged as a dominant input and were fit to a single exponential growth curve with $\mathrm{T}=2.7$ days (Fig. 29B).

Rate of calyx growth. To further explore the growth dynamics of this system, averages of the largest inputs onto each cell body at ages P2-6 were used to generate a growth curve for the populations of sampled cells (Fig. 29C). A Boltzmann fit to the data revealed a half-maximal age $\left(\mathrm{t}_{1 / 2}\right)$ of 3.5 days at a calyx size of $145 \mu \mathrm{m}^{2}$. The maximum growth rate (dashed curve in Fig. 29C) at $t_{1 / 2}$ was $207 \mu m^{2}$ per day. Note that this description of population growth dynamics may underestimate the growth rate of individual calyces. Additionally, ASA was compared across ages using non-parametric statistical methods (see Methods). The daily increase in mean size from P2-4 was highly significant $(Z=6.64, p<0.0001$, non-parametric Jonckheere-Terpstra Test, see Methods). In follow-up individual comparisons, the mean for P2 was significantly smaller than P3 $(p<0.0001)$, which in turn was significantly smaller than P4 $(p=0.0003)$. Previously it was demonstrated that maturation of MNTB cells parallels calyx growth (Hoffpauir et al., 2010; Rusu and Borst, 2011), although the population dynamics of calyx growth could only be estimated from physiological measures and serial section EM at the single time point of P4 (Hoffpauir et al., 2006). Boltzmann fits to physiological data from Hoffpauir et al. (2010) are re-plotted with anatomical measures of calyx growth in Fig. 29D. Both the transition from tonic to phasic firing and the increase in action potential (AP) current threshold begin about one day earlier than growth of calycigenic terminals. The half maximal times for percent phasic cells $\left(t_{1 / 2}\right.$ $=3.5$ days) is identical to calyx growth and the value for increase in AP current threshold is less than one day delayed $\left(\mathrm{t}_{1 / 2}=4.4\right.$ days $)$.

Correspondence between electrophysiological and anatomical counting methods. Because these ultrastructural data reveal all inputs onto the MNTB cell, we used them as a template to similarly analyze physiological measures of input number and synaptic current amplitude from 
Hoffpauir et al. (2010). Since minimal stimulation techniques revealed fewer inputs per cell and there were fewer inputs measured physiologically per age, we combined ages P2-4 to capture the transition in input size and generate a histogram of evoked EPSC amplitudes. This histogram had a noticeable gap at $500 \mathrm{pA}$, which we defined as differentiating large from small inputs (Fig. 30A). This value was intermediate between threshold values ( 400 pA to $1 \mathrm{nA}$ adjusted for holding potential) for calyceal inputs used in studies of older animals, which have fewer small inputs (Forsythe and Barnes-Davies, 1993; Futai et al., 2001; Bergsman et al., 2004). For cells with at least one large input, we next calculated the ratio of EPSCs separately for each age. At P3, 31\% (5/16) of cells had at least one large input, and at P4 this value increased to $78 \%(18 / 23)$. The fraction of cells with at least one large input was slightly less than values obtained using SBEM ( $41 \%$ at $\mathrm{P} 3,100 \%$ at $\mathrm{P} 4)$, consistent with the notion that some inputs are cut during brain slice preparation or that some inputs have similar thresholds, resulting in underestimates of cells having large inputs. We next calculated the input size ratio, as with anatomical data, as a metric for cells that had an established dominant input. At P3 and $\mathrm{P} 4$, the input size histograms had a break at values of 4 and larger, similar to anatomical data, to differentiate cells with actively competing inputs from cells with a dominant input (Fig. 30A', A"). At P3, $2 / 16(12.5 \%)$ cells had a dominant large input and at P4 this fraction increased to $12 / 23(52 \%)$. As with the other physiological parameters, these values were less than anatomical measures (Fig. 30B).

\section{Innervation territories of competing inputs are segregated}

As calyx-forming inputs grow, they establish new synapses with the MNTB neuron. At the NMJ, postsynaptic receptor sites are mapped onto the muscle surface, are relatively invariant in position although they grow in size (Balice-Gordon and Lichtman, 1990), and innervation of receptor loci can be traded between competing inputs (Walsh and Lichtman, 2003). For CF:PC innervation, small inputs are intermingled on the cell body before the eventual "winning" input 
extends over the dendritic end of the cell body in the capuchon stage of terminal development (Sugihara, 2005; Hashimoto et al., 2009). We investigated locations of competing inputs on the MNTB somatic surface to determine if competing inputs were interdigitated, which would indicate that they vied for the same postsynaptic territory, or if they were segregated as eventually occurs at the CF:PC. The ASA and glial incursions for all actively competing inputs onto cells at P3-6 were mapped onto the postsynaptic surface and inspected for innervation of contiguous regions of the somatic surface. In all cases, actively competing inputs occupied segregated territories on the somatic surface (Fig. 31). The ASAs for three smaller, actively competing inputs (P3 cell, Fig31A) indicate that they begin their growth within wellcompartmentalized regions of the somatic surface. This segregation is maintained as competing inputs grow (different cell from P3 depicted in Fig. 31B) and can be located on opposite sides of the cell body (P6 cell, Fig. 31C). The closest contact between competing inputs in our data set is illustrated in Fig. 31B (arrowhead), where extensions from the main region of ASA for each input nearly touch but remain on opposite sides of a somatic spicule. We next investigated whether glial incursions (white arrowheads, Fig. 23C) between calycigenic terminals and the somatic surface were in contact, considering that these glial processes better indicate the actual edges of the terminals and may mediate physical interactions. The contact areas between these glial incursions and the somatic surface are indicated in Fig. 31A', B' and C'. These regions also remain segregated on the somatic surface.

\section{Discussion}

With this systematic analysis of high-resolution structural images, we demonstrate that the innervation of MNTB principal neurons bears the hallmarks of initial exuberance and strengthening and pruning characteristic of other developing neural systems. A well-timed growth spurt exceeding $200 \mu \mathrm{m}^{2} /$ day during a single day at P3 is more constrained temporally than in other systems, such as CF:PC and NMJ, and offers opportunities to study growth and 
competition on a compressed time scale. In retrospect, this characteristic made possible this first application of SBEM to the developing brain at high temporal resolution.

\section{Experimental considerations}

We relied upon the distribution of terminal sizes to define large inputs (greater than 25 or 35 $\mu \mathrm{m}^{2}$ depending upon age), and the presence of at least two large inputs as an estimate (50\% at P3) for the prevalence of competition. This is a minimal estimate because our 24-hour sampling interval may miss a stage at which more MNTB cells are contacted by competing inputs, due to explosive growth of calycigenic terminals at P3. Note that changing the threshold for defining large inputs does not change appreciably the prevalence of competition (60\% at $15 \mu \mathrm{m}^{2}$ to $43 \%$ at $35 \mu \mathrm{m}^{2}$ ), so this metric is not sensitive to the method of defining threshold nor its small variation across age. Therefore, we propose a qualitative model that incorporates both competition among large inputs and emergence of only a single large input from initial exuberant innervation (Fig. 32A).

We provided a refined definition of active competition based upon the input size ratio, considering that a large input may have already lost the competition to a much larger input. Additional rules may have better defined cells with actively competing inputs. For example, the largest input for some cells could be $>100 \mu \mathrm{m}^{2}$ larger than the second largest input, and considered to be the dominant large input; however, the ratio of input sizes $(<5)$ placed the cell into the actively competing category. Conversely, some cells had a single large input, classified as a dominant large input, that was relatively small $\left(<50 \mu \mathrm{m}^{2}\right)$, therefore close in size to the second largest input with which it could be in active competition. Very few cells and approximately equal numbers fell into these complementary borderline categories ( 1 in each category at P3 and P6; 2 in the first and 1 in the second category at P4), so this concern does not alter our conclusions. 
The use of SBEM permitted well-defined metrics for synaptic growth and competition because it revealed actual apposed surface area (ASA). Light microscopic labeling of the calyx, including immunolabeling for synaptic vesicle proteins, overestimates its size due to glial incursions between the calycigenic terminals and MNTB cell (Fig. 23C; Rowland et al., 2000). SBEM also permitted tracking fine processes due to precise alignment between sequential images. The morphology of these inputs is in general agreement with previously published descriptions of the protocalyx and immature calyx (Morest, 1968a; Kandler and Friauf, 1993), but with more accurate $3 \mathrm{D}$ reconstruction due to efficient capture of thin processes. One limitation of SBEM is that the tissue volume is smaller than in some light microscopy studies, although our more recently collected image volumes (P2, P6 and P9, Table 1) bridge this gap. Note that our analysis is more extensive and time-consuming than in previously published studies using SBEM or related techniques (Bock et al., 2011; Briggman et al., 2011), because we quantify contacts by ASA in 3D. One goal of nanoscale connectomics is to compare wiring diagrams between brains in different conditions (Seung, 2012). We have achieved an expression of this goal by comparing the same brain region at short temporal intervals across developmental age.

\section{The nature of competition}

Our data place constraints on the $\mathrm{CH}$ :MNTB competitive process and reveal similarities with other model systems. We were often able to trace small inputs at P2 to large diameter, calycigenic axons, noting that some inputs not traced to calycigenic axons may represent GABAergic or glycinergic inputs (Awatramani et al., 2005b; Hoffpauir et al., 2010; Rusu and Borst, 2011). Convergence of at least 3-4 small inputs from calycigenic axons is evidence for initial exuberance because only 2-3 inputs strengthen to pass through a second pruning phase for monoinnervation. This process is similar to the RGC:dLGN, CF:PC and NMJ systems, whereby tens of small inputs winnow to 2-3 (NMJ) or 5-7 larger inputs (CF:PC, NMJ) during a first phase of competition by strengthening a subset of inputs while pruning many others 
(Chedotal and Sotelo, 1992; Colman et al., 1997; Chen and Regehr, 2000; Sugihara, 2005; Tapia et al., 2012). After this first phase of strengthening and pruning in these systems, small inputs do not participate further in competition. Competition resolves to monoinnervation for most MNTB cells within 2 days, similar to rapid emergence of a dominant input in the CF:PC system during the "nest" stage of innervation between P4-7 (Kano and Hashimoto, 2012). In the RGC:dLGN and NMJ systems, this final phase of competition is protracted, resolving over 1-2 weeks; growth of CFs along with the PC dendritic tree occupies a similar period of time (Brown et al., 1976; Balice-Gordon and Lichtman, 1993; Chen and Regehr, 2000).

Competition for the final $1 / 3$ of MNTB cells resolves more slowly and is complete by P9 in all but a small subset of cells, consistent with physiological measures at P9-11 (Bergsman et al., 2004). This group was spatially clustered at the margins of the MNTB; and may reveal a small subpopulation of MNTB cells that proceeds at a different developmental pace, remains multiinnervated or disappears after hearing onset. These cells may elude detection by light microscopy in part because the summed area of all inputs can be large $\left(>200 \mu \mathrm{m}^{2}\right)$.

Our data also place a size limit on competitive inputs. At each age, at least one cell in the sample volume was contacted by two very large inputs of nearly equal size (Figs. 25C,F,F'; 26B,E, 27C,E) but these were never the largest inputs. Perhaps both competing inputs grew to such a large size in few cases, or elimination of "losing" inputs is a swift process. At the NMJ, the smaller of two large inputs can eventually out-compete the larger in a process referred to as "flip-flop" (Walsh and Lichtman, 2003). We cannot rule out this possibility for calyx innervation, but it is unlikely for cells that resolve a dominant input by P4 due to the rapid growth rate. For cells in which competition resolves more slowly after P4, "flip-flop" of actively competing inputs is more likely. In contrast to the NMJ (Bishop et al., 2004; Turney and Lichtman, 2012), once an axon has withdrawn contact from an MNTB cell, it is difficult to identify within the complex 
cellular arrangements of the MNTB. Processes of degeneration and their resemblance to other systems (Luo and O'Leary, 2005) will be presented in a subsequent report.

\section{Potential mediators of calyx growth and competition}

Calyx growth is integrated with other aspects of tissue reorganization in the MNTB. At P2, MNTB neurons are tightly packed, with much of their somatic surface apposed to other neurons or wrapped by glia. Somatic contact between principal cells and glial wrapping of the somatic surface is less prevalent at P3 and provides space for large terminals to innervate the somatic surface. These structural changes may be part of a process whereby neurons alter the composition of their cell membrane to disentangle from other cells and prime for synapse formation. Postsynaptic structural changes may form part of the maturation process, such as an apparent increased number of somatic spicules (compare Fig. 24B, inset with Fig. 27E). Calyx growth closely parallels physiological transitions in the MNTB cell (Fig. 29C; Hoffpauir et al., 2010) suggestive of common initiating events. One trigger for these coordinated pre- and postsynaptic events may be transition from non-bursting spontaneous activity (Marrs and Spirou, 2012) to bursting patterns initiated in the cochlea and delivered by globular bushy cells to the MNTB (Tritsch and Bergles, 2010; Tritsch et al., 2010; Fig. 32B).

Evidence across multiple neural systems has accumulated for activity-dependent selection of competitive winners (Balice-Gordon and Lichtman, 1993; Buffelli et al., 2003; Hooks and Chen, 2006; Ohtsuki and Hirano, 2008; Favero et al., 2012) that triggers through cell signaling pathways involving protein kinases (reviewed by Watanabe and Kano, 2011) and which may be subject to global factors such as metabolic load of the presynaptic neuron (Kasthuri and Lichtman, 2003). Actively competing calyces are also growing, indicating a level of support for both inputs by the MNTB cell. In contrast to the NMJ, where axons vie for a fixed postsynaptic receptor area (Turney and Lichtman, 2012), competing calycigenic inputs are segregated on the somatic surface (Fig. 31). This spatial organization may facilitate reinforcement via a Hebbian 
mechanism of inputs that stay ahead of the rapid and concurrent increase in action potential threshold. Location of calyx-forming inputs on the somatic surface may be a determinant of the eventual winner, similar to early designation of the winning CF as the first to innervate the dendritic pole of the PC cell body (Hashimoto et al., 2009). Further investigation should include assessment of $\mathrm{CH}$ :MNTB growth dynamics across the entire frequency axis of the MNTB so that local competing mechanisms can be compared with global mechanisms, such as mismatched chemical signals such as Ephs and ephrins (Hsieh et al., 2010; Nakamura et al., 2012), as has been studied at the NMJ (Tapia et al., 2012).

In summary, the $\mathrm{CH}$ :MNTB shares features with other model systems for neural development. A unique feature for the $\mathrm{CH}$ :MNTB is the more rapid occurrence of these developmental events and their completion before the onset of sensitivity to airborne sound between P10-12 (Ehret, 1976; Romand and Ehret, 1990; Kamiya et al., 2001; Sonntag et al., 2009). This compressed time frame has made possible precise quantification of population growth dynamics and may offer experimental advantages to decipher cellular mechanisms for synaptic growth and competition. 


\section{Chapter 3: Terminal Growth Occurs Asymmetrically with Respect to Nuclear Position during Development of the Calyx of Held in the Mouse}

Paul S. Holcomb ${ }^{1}$, Sara Berzingi ${ }^{4}$, Cody L. Mullins ${ }^{4,5}$, Jessica L. Patterson ${ }^{4}$, Michael Morehead ${ }^{6}$, Thomas J. Deerinck ${ }^{7}$, Mark H. Ellisman ${ }^{7,8}$, George A. Spirou ${ }^{1,2,3}$

${ }^{1}$ Rockefeller Neuroscience Institute, Departments of ${ }^{2}$ Otolaryngology, and

${ }^{3}$ Physiology and Pharmacology, ${ }^{4}$ West Virginia University School of Medicine, Morgantown, WV 26506-9303, USA

${ }^{5}$ Perelman School of Medicine, University of Pennsylvania, Philadelphia, PA 19104

${ }^{6}$ Lane Department of Computer Science and Electrical Engineering, Benjamin M. Statler College of Engineering and Mineral Resources, West Virginia University, Morgantown, WV, 26506-6070, USA

${ }^{7}$ National Center for Microscopy and Imaging Research and ${ }^{8}$ Department of Neuroscience, University of California, San Diego, California 92092-0608, USA 


\section{Abstract}

Polarity is a vital aspect of the development and function of the nervous system, but little is known about how intracellular organelle arrangement—especially nuclear eccentricity—may influence innervation. The calyx of Held:MNTB principal cell connection has emerged as a useful model system for studying terminal formation due to its rapid maturation, large terminal size, and monoinnervated endpoint. Using serial block-face scanning electron microscopy and 3D reconstruction of neurons and associated structures, we show that principal cells possess eccentric nuclei, and that terminals selectively innervate and grow on the cell surface opposite the nucleus position. Mechanisms for biasing terminal growth, such as asymmetric addition of new membrane during principal cell growth, glial coverage of the principal cell, and polarized location of dendrites were also assessed. Principal cell surface expansion was significantly higher on the side of the cell being innervated at P4 and P6, owing mostly to an expansion of small cellular projections called somatic spicules. Glial processes were found to cover a significant portion of the principal cell surface (49-57\%, P2-P6), and showed a bias toward coverage of the cell surface nearest the nucleus at P4. Bias was also evident in dendritic location; approximately $70 \%$ of all dendrites were found to reside on the side of the cell opposite the nucleus. This analysis also revealed a two-stage process of dendritic pruning that may have implications for principal cell innervation and terminal competition. 


\section{Introduction}

Polarity is a major factor in all aspects of neural development: mitosis and cell fate determination, migration, axon and dendrite specification, and even synaptogenesis. Morphological polarity is evident at multiple levels of neuronal organization. At the tissue level, it can be seen in the polarized alignment of hair cells in the inner ear essential for sound transduction. The structure of axons and dendrites ensuring the flow of information in neurons demonstrates the most recognizable aspect of neuronal polarity at the cellular level. Finally, the positioning of the centrosome with the nucleus guaranteeing the correct division of nuclear material during mitosis is but one of many examples of vital intracellular polarity programs (Holcomb et al., 2013a). Polarized neuronal innervation has been considered in respect to dendrite-axon alignment, but much less focus has been placed on the polarized arrangement of internal organelles and how this may affect innervation in the developing nervous system.

The connection between the principal cell of the medial nucleus of the trapezoid body (MNTB) and the calyx of Held $(\mathrm{CH})$-one of the largest terminals in the mammalian central nervous system—has emerged as an excellent model system for examining terminal development and competition. This is due to its rapid maturation (48-72 hours), large terminal size, and monoinnervated endpoint (Hoffpauir et al., 2010; Holcomb et al., 2013b). Many studies have observed an eccentrically placed nucleus within the MNTB principal cell, and qualitative observations of terminal location have suggested that calyx placement may be polarized with respect to the cell nucleus (Bollmann et al., 1998; Hoffpauir et al., 2006; Rodríguez-Contreras et al., 2006; Holcomb et al., 2013a, 2013b). These observations are particularly intriguing due to the apparent lack of directionality in the organization of the MNTB as a whole; the presence of a polarity program to dictate terminal placement in a non-laminar system would be a novel finding with potential implications for understanding terminal development in other similar brain regions. 
The use of serial block-face scanning electron microscopy (SBEM) in describing $\mathrm{CH}$ and MNTB principal cell morphology has proven highly effective previously (Holcomb et al., 2013b). SBEM-a process for gathering serial EM images with both high resolution (4-10nm) and exceptional alignment between images_allows for the unambiguous identification of cellular components and tracking of processes over long $(\mu \mathrm{m})$ distances. The segmentation and reconstruction of structures from these images allows for a quantitative analysis of neuronal development unmatched in other imaging techniques. To ensure the most accurate measurement of polarity and terminal placement possible, we utilized SBEM to assess these and other morphological features of the developing $\mathrm{CH}$ and MNTB principal cell in 24-hour intervals from P2-P9.In doing so, this study codifies for the first time the morphological characteristics of polarity in the developing CH:MNTB connection, including changes in cell structure that may have implications in competition during $\mathrm{CH}$ formation. 


\section{Materials and Methods}

\section{Ethical Approval}

All procedures involving animals were approved by the West Virginia University (WVU) Institutional Animal Care and Use Committee and were in accordance with policies of the United States Public Health Service.

\section{Animals}

A mouse breeding colony (FVB/NJ; The Jackson Laboratory, Bar Harbor, ME, USA or FVB/NCr, National Cancer Institute, Frederick, MD, USA) was housed in the vivarium at WVU School of Medicine. Mice were paired overnight to accomplish timed pregnancies, with fertilization assumed to have occurred at approximately midnight. Typically, birth occurred overnight, with an average gestation time of approximately 19 days (E0-E18). Ages are accurate within 12 hours, as mice were checked twice daily.

\section{Serial Block-face Electron Microscopy (SBEM)}

Tissue was processed and imaged as published previously (Deerinck et al., 2010; Holcomb et al., 2013b). Cells were segmented, reconstructed and analyzed using either manual segmentation in the IMOD 3D reconstruction and visualization software (Boulder Laboratory for 3-D Electron Microscopy of Cells, University of Colorado: Boulder) or a combination of initial segmentation with the carving workflow in the llastik software (Heidelberg Collaboratory for Image Processing , University of Heidelberg, Germany) and proofreading and reconstruction using the Seg3D software (Scientific Computing Institute, University of Utah). Cells were omitted from reconstruction if a portion of the cell body was truncated by the bounds of the data set, or if enough of an input extended outside of the data volume to prevent its identification as a distinct input. The nucleus, cell body and all neural inputs were segmented for 107 cells across the 
postnatal ages studied. A large tracing team (30+ people) completed the segmentations. Accuracy of segmentation was verified as detailed previously (Holcomb et al., 2013b). Apposed surface areas were traced at the contact between each presynaptic input and its postsynaptic partner (ASA) or glial process (gASA); these contacts were identified during segmentation and either segmented and meshed using the IMOD meshing tool or reconstructed using Boolean intersections of masks representing the terminal (or glial process) and cell body segmentations in Seg3D. Both methods created a two-dimensional sheet-like surface, which was used as a representation of terminal location and strength in the case of the ASA. Morphological measurements were performed either using Rhinoceros (McNeel, Seattle, WA) or MeshLab (Visual Computing Lab, Istituto di Scienza e Tecnologie dell'Informazione, Pisa, Italy), calculations were performed using Microsoft Excel (Microsoft, Redmond, WA), custom Python scripts, or MATLAB (The MathWorks, Inc., Natick, MA). MATLAB was also used to generate all graphs.

\section{Model conversion and alignment}

For cells reconstructed with IMOD, the cell models were converted from IMOD format into OBJ format using the imod2obj command in the IMOD terminal interface. Cells reconstructed with Seg3D were output into VTK format and converted to OBJ format using a custom Python script. These converted models were then imported into the Rhinoceros CAD (RhinoCAD) software (McNeel, Seattle, WA). The number of faces for each object (nucleus, cell body) was then standardized to 20,000 and 40,000 , respectively, to unify the model characteristics from the two different reconstruction methods and to reduce calculation time; this reduction did not change surface area or volume characteristics by more than $5 \%$ for any model. Centroids of the nuclei and cell bodies of reconstructed cells at ages P3-P9 were computed using the "VolumeCentroid" command within RhinoCAD. Models were translated such that the cell centroid was located at the origin, and the model was rotated with respect to the origin such that 
the nucleus centroid was located along the $-Z$ axis. This translation and rotation established the "polarity vector" between the nucleus centroid and cell body centroid. Due to the smaller cell body and more fusiform cell shape at P2, however, cell body centroid locations were biased such that the nucleus was not oriented similarly to other ages. Due to this, a different polarity alignment technique was used. For all P2 cells, the primary axes of rotation for the nucleus were calculated using the MeshLab software (Cignoni et al., 2008). Each principal cell was then rotated such that the nucleus third axis of rotation (shortest axis) lay along the Z-axis, with the majority of the nucleus volume located in $-Z$, similar to other ages studied. Nuclear and cell body volume and surface area were computed using the "Volume" and "Area" commands within RhinoCAD.

\section{Nuclear eccentricity measurement}

Two methods were employed to define nuclear eccentricity. First, a custom Python script was written to measure the distance from each vertex comprising the nucleus OBJ to each vertex of the associated cell body and return the minimum of these distances. Minimum distances were binned in $25 \mathrm{~nm}$ increments, and the resulting histograms for all nucleus-cell pairs at each developmental age were averaged. Second, the distance along the polarity vector (Z-axis following alignment) from the centroid of the nucleus to the cell membrane in both directions was measured within RhinoCAD. These distances were then compared to quantify nuclear eccentricity.

\section{Terminal and Glial Placement Measurement}

To quantify terminal placement, neurons were binned in $1 \mu \mathrm{m}$ increments by planes perpendicular to the polarity vector and symmetric about the cell body center (10 bins in the cytoplasmic pole, 10 bins in the nuclear pole). The amount of surface area of the ASA for each of the three largest terminals was quantified per bin, and measurements were combined into cumulative area curves. Bins were expanded in the radial direction on a cell-by-cell basis to 
guarantee complete capture of the ASA; if ASAs extended beyond the bins in the direction of the polarity vector, the additional surface area was included in either the first bin for the cytoplasmic pole or last bin for the nuclear pole. Nucleus volume was also binned in this manner to provide a reference for the nuclear pole and as a tertiary measure of nuclear eccentricity. Glial process placement was quantified by dividing the gASA into cytoplasmic and nuclear pole components using a single plane perpendicular to the polarity vector and passing through the cell center.

\section{Simulation of Terminal Placement}

Random terminal placement and polarity quantification was accomplished through a custom Python script using the Visualization Tool Kit (VTK, Kitware, Clifton Park, NY). A cell body reconstruction representative of the average postnatal day 4 cell in volume and surface area $\left(1626 \mu \mathrm{m}^{3}\right.$ volume, $1540 \mu \mathrm{m}^{2}$ surface area) was used for the simulation; average cells from P2 and P3 were also simulated, with no statistical difference in outcome. A mesh face was selected at random, and the terminal grew isometrically by agglomerating neighboring faces on the mesh until the average largest terminal apposed surface area at P4 $\left(249 \mu \mathrm{m}^{2}\right)$ was exceeded.

Terminal placement was then assessed as described for EM reconstructions. One thousand simulation results were averaged to achieve a distribution of apposed surface area for random terminal placement that was compared to results from EM reconstructions.

\section{Terminal Comparison and Logistic Fit}

To compare cumulative area curves from binned input measurements to either one another or to simulation results of random terminal placement, the JMP software (SAS Institute Inc., Cary, NC) was used to fit the measured curve data based on a modified logistic curve:

$$
y=\theta_{1}+\left(d_{1} * I_{1}\right)+\frac{\theta_{2}-\theta_{1}+\left(d_{2} * I_{1}\right)}{1+e^{\left(\theta_{3}+\left(d_{3} * I_{1}\right)\right) *\left(x-\theta_{4}+\left(d_{4} * I_{1}\right)\right)}}
$$


In this equation, $\theta_{1}$ and $\theta_{2}$ represent the upper and lower sills of the curve, respectively, while $\theta_{3}$ is the maximum steepness of the curve (measured at the midpoint) and $\theta_{4}$ is the value of $x$ at the midpoint of the curve. The variables $d_{1-4}$ represent the deviation from the initial curve (as defined by $\theta_{1-4}$ ) necessary to fit the curve being compared. $l_{1}$ is a binary operator that determines if the variables $d_{1-4}$ are to be used (Fig. 33). To compare two curves, first $l_{1}$ is set to zero, eliminating the $d_{1-4}$ variables. The resulting equation is used to fit the measured data for one of the two curves being compared (typically the simulation results as discussed in the previous section) using JMP, and corresponding values for $\theta_{1-4}$ are found. Using these values as initial conditions and returning to the original equation, the second curve is fit by fixing $\theta_{1-4}$ and allowing the variables $d_{1-4}$ to be varied until the best fit to the curve is found, again using JMP. These values $\left(d_{1-4}\right)$ representing the deviation of fit between the two curves and their associated $95 \%$ confidence intervals provide the basis for the statistical comparison between polarity curves.

\section{Poisson Reconstruction and Somatic Spicule Surface Area Quantification}

3D reconstructions of cell bodies were processed in MeshLab using a Poisson surface reconstruction approach codified by Kazhdan et al. to create a smoothed representation of the cell body and remove somatic spicules (Kazhdan et al., 2006). Briefly, the goal of Poisson reconstruction is to define an indicator function ( 1 inside the model, 0 outside) from a set of points in 3D space and use this to create an isosurface that approximates the shape of the object these points represent. Kazhdan et al. revealed a relationship between the gradient of the indicator function $(\nabla \chi)$ and the normal of the points comprising the surface: $\nabla \chi$ represents a vector field that is zero except at the surface points where its value equals the point's inward facing normal (Kazhdan et al., 2006). Given this, the indicator function can be found by determining the scalar function $\chi$ that is closest to the vector field created by the surface points $(\vec{V})$. This problem simplifies into: 


$$
\nabla^{2} \chi=\nabla \cdot \vec{V}
$$

which is in the form of Poisson's equation. The Poisson reconstruction technique is implemented by discretizing the surface points using an adaptive octree where each node has eight children (or "leafs"). A salient feature of this approach is that controlling the octree depth allows for control of the resolution of the resulting isosurface. Knowing this, octree depth was optimized for each cell to minimize the difference in volume between the original mesh and smoothed reconstruction while removing surface projections; all Poisson reconstructions were within at least $5 \%$ of the original cell body volume. Poisson reconstructions were then imported into RhinoCAD, and morphological metrics (surface area, volume, etc.) were calculated as for the original cell reconstruction. The difference in surface area between the original and Poisson reconstruction was considered the surface area of somatic spicules.

\section{Statistical Analysis}

All data is presented as mean \pm standard deviation. Statistical significance was defined as a $p$ value $<0.05$. Data was tested for normality using the expanded Shapiro-Wilk test. Due to size differences skewed toward older ages, as well as a high degree of variance in developmental state on a cell-by-cell basis, non-parametric statistical methods were used. Age and developmental comparisons of distance, volume, and surface area were accomplished in Microsoft Excel (Microsoft, Redmond, WA) using the non-parametric Kruskal-Wallis H-test and-if statistical significance was obtained-a pairwise comparison between groups was accomplished using the Mann-Whitney U-test. Statistical comparison of counts for axondendrite association was performed using a Chi-squared test. Comparison of polarity curves was achieved by computing a $95 \%$ confidence interval for each variable $d_{1-4}$ (the change in the initial condition necessary to fit the curve). If this confidence interval does not include zero—that is, if the original curve fit does not fall within the confidence interval—the two parameters were said to be significantly different. The p-value for the polarity curve data was calculated by 
estimating the standard error from the $95 \%$ confidence interval, calculating a t-statistic and determining p-value using the following equation in the JMP software (SAS Institute Inc., Cary, NC):

$$
(1-\operatorname{tstatistic}(\operatorname{Maximum}(t,-t), d f)) * 2
$$

where "Maximum" (a built-in function of JMP) guarantees a positive t-statistic, df is the degrees of freedom (number of samples - 1), "tstatistic" (also a built-in function of JMP) returns the probability of the t-statistic at the given df. The result of the equation is multiplied by 2 for a twotailed t-test. 


\section{$\underline{\text { Results }}$}

\section{Principal Cell Nuclei are Asymmetrically Localized}

Qualitative observations of SBEM image stacks at multiple ages $(P 2,3,4,6,9,30)$ from our previous studies and others have suggested that MNTB principal cells have asymmetrically localized nuclei (Fig. 34A; Hoffpauir et al., 2006; Holcomb et al., 2013a; Rodríguez-Contreras et al., 2006). To demonstrate this quantitatively, we utilized 3D reconstructions of principal cell nuclei and cell bodies reconstructed from SBEM segmentation. The linear distance from each vertex of the 3D reconstruction of the principal cell nucleus to all vertices of the cell body reconstruction was measured to find the minimum distance, and the results of all cells measured within a single age were averaged. Measurement results for ages P2-P9 were heavily skewed towards sub-micron distances, with $>50 \%$ of minimum distances at ages P2-P6 and $>40 \%$ at P9 falling below $1 \mu \mathrm{m}$ (Fig. 34B). In many cases, the distance between the nuclear and cell body membranes was so small that the difference was almost indistinguishable in electron microscopy images (Fig. 34C-D). At P30, well after development of the calyx of Held and onset of hearing ( P12; Ehret, 2005; Hammerschmidt et al., 2012), eccentricity of the nucleus appears to relax, with only $24 \%$ of minimum distances below $1 \mu \mathrm{m}$ (Fig. 34B-dark orange, Fig. 34E). These results show that nuclear eccentricity is a ubiquitous characteristic of MNTB principal cells throughout early developmental ages, a feature we have termed "intrasomatic polarity", and suggest that eccentricity may lessen at later ages when development has completed.

To further examine intrasomatic polarity—both within and across developmental ages—it was first necessary to define a three-dimensional alignment that would provide a single reference frame for all cells. To do this, the centers of geometry of the nucleus and cell body were used as control points to align each cell. The center of the cell body was defined as the origin $(0,0,0)$ and a vector from the nucleus center to the cell body center defined the major $(Z)$ axis of each cell (Fig. 35A) except for P2 cells (see Methods). A perpendicular plane (XY) passing through the 
cell body center bisected the cell into a "cytoplasmic pole" $(\mathrm{CP})$ in $+Z$, and a "nuclear pole" (NP) in $-Z$. Within this reference frame, a secondary measurement of nuclear eccentricity was performed by comparing the distance from the nucleus center to the cell membrane in either the cytoplasmic pole (N-CP) or nuclear pole (N-NP) along the Z-axis. For centric nuclei, these two distances should not be significantly different. In contrast, all MNTB principal cells displayed significant differences between N-CP and N-NP lengths across all developmental ages studied, with the N-CP length larger in every case $(4.82 \pm 0.6 \mu \mathrm{m} N-N P$ vs $10.9 \pm 2.7 \mu \mathrm{m} N-C P, P 2-P 9$ combined, $n=97$ cells (11 at $P 2,29$ at $P 3,17$ at $P 4,29$ at $P 6$, and 11 at $P 9$, one brain per age), $p=8.45 \times 10^{-33}$, Mann-Whitney U-test (M-W), Fig. 35B). Additionally, although there was no significant change in N-NP distance from P2-P9 $(p=0.051$, Kruskal-Wallis H-test $(K-W))$, the NCP distance increased significantly from $8.83 \pm 3.5 \mu \mathrm{m}$ at $\mathrm{P} 2(\mathrm{n}=11)$ to $11.89 \pm 2.7 \mu \mathrm{m}$ at $\mathrm{P} 4(\mathrm{n}=17)$ $(p=0.003, M-W)$. The difference in N-CP distance between P2 and later ages (P6-P30) remains significant, but no significant difference was found in N-CP in pairwise comparisons of P3-P9, suggesting that there is a rapid outgrowth and then stabilization of intracellular distance during this stage of development. By P30, both N-NP and N-CP distances have increased compared to $\mathrm{P9}(6.36 \pm 2.1 \mu \mathrm{m}$ and $13.92 \pm 2.1 \mu \mathrm{m}, \mathrm{n}=8$ cells $)$ but only the $\mathrm{N}-\mathrm{CP}$ change reached the level of significance $(p=0.019$ for $N-C P, p=0.069$ for $N-N P, M-W)$. These results confirm our previous analysis of nuclear eccentricity and suggest that intrasomatic polarity increases during the developmental window studied.

There are multiple mechanisms that may increase intrasomatic polarity. First, the nucleus may move within the cell into a more eccentric position. Although the consistency of the nucleus-tocell membrane minimum distance (Fig. 34B) and N-NP distance (Fig. 35B) measures suggest this is not the case, we cannot rule out nuclear movement due to the extended time between our time points and static nature of imaging in electron microscopy. Second, nuclear eccentricity may also appear to increase if the nucleus shrinks as development progresses. Contrary to this, 
volumetric analysis of nuclei showed a small, transient, but significant increase in nuclear volume across ages P2-P9 $\left(453 \pm 58 \mu \mathrm{m}^{3}\right.$ at $\mathrm{P} 2$ to $505 \pm 19 \mu \mathrm{m}^{3}$ at $\mathrm{P} 4, \mathrm{p}<0.05, \mathrm{M}-\mathrm{W}$; decrease from P4 to $482 \pm 29 \mu \mathrm{m}^{3}$ at P6, $\mathrm{p}=0.004, \mathrm{M}-\mathrm{W}$; Fig. 35C arrows indicate comparison). Finally, eccentricity may increase if the cell body expands away from the nucleus, thus shifting the location of the cell center further from the nucleus center. Volumetric analysis of the cell body showed a significant increase in cell volume with respect to age except for P4-P6 (average of $1012 \pm 190 \mu m^{3}$ at $P 2(n=14)$ to $1989 \pm 212 \mu m^{3}$ at $P 9(n=11) ; p=1.41 \times 10^{-12}, K-W$, Fig. $\left.35 C\right)$. As the percentage of cell volume occupied by the nucleus declines from $45.1 \pm 3.5 \%$ at P2 to $24.3 \pm 1.5 \%$ at P9, nuclear growth and cell growth appear decoupled during early development. In contrast, cell volume occupied by the nucleus remained constant between P9 and P30 $(23.9 \pm 1.9 \%)$, with a significant and symmetric increase in both nucleus and cell body volume between these ages. Cell body growth, coupled with the significant increase in N-CP distance, suggests that directed expansion of the cell body away from the position of the nucleus from P2 (Fig. 35D) to P4 (Fig. $35 \mathrm{E})$ accounts for the increase in nuclear eccentricity during development.

Previous work has shown similar time courses for structural changes in calyx growth and electrophysiological changes in the MNTB principal cell, suggesting a coordinated pre- and postsynaptic maturation of this connection (Hoffpauir et al., 2010; Holcomb et al., 2013b). We have previously described three developmental stages for calyx growth: cells with all small inputs $\left(<25 \mu \mathrm{m}^{2}\right)$ were defined as "small", cells with at least 2 competing inputs $\left(>25 \mu \mathrm{m}^{2}\right.$, ratio of two largest input sizes < 5) were defined as "competing", and cells with a single dominant input $\left(>25 \mu \mathrm{m}^{2}\right.$, ratio of two largest input sizes $>5$ ) were defined as "dominant" (Holcomb et al., 2013b). Although all of these developmental stages may be found in MNTB principal cells at a single age, there is a distinct correlation between postnatal time point and developmental status; with a very small percentage of exceptions, no cells with only small inputs are found after P3, and no cells possess a dominant input at P2 (Hoffpauir et al., 2010; Holcomb et al., 2013b). To 
determine if the polarity found in our developmental categories stems solely from differences in developmental time point, we restricted our analysis to cells at P3-an age when all developmental categories are present (small: n=13, Fig. 36A; competing: n=9, Fig. 36A'; dominant: n=7, Fig. 36A"). All groups showed significant difference between N-NP and N-CP distances at $\mathrm{P} 3$ (small: $p=7.34 \times 10^{-6}$, competing: $p=7.85 \times 10^{-5}$, dominant: $p=8.72 \times 10^{-4}, M-W$ ). No significant difference was found in N-NP between all developmental groups $(p=0.184, K-W)$ or between N-CP in competing and dominant cells $(p=0.356, M-W)$. However, a significant difference was found in N-CP between small and competing cells $(9.19 \pm 2.3 \mu \mathrm{m}$ versus 11.01 $\pm 2.1 \mu \mathrm{m}, \mathrm{p}=0.018, \mathrm{M}-\mathrm{W})$ and N-CP between small and dominant cells trended toward significance $(9.19 \pm 2.3 \mu \mathrm{m}$ versus $10.96 \pm 3 \mu \mathrm{m}, \mathrm{p}=0.061, \mathrm{M}-\mathrm{W})$ (Fig. 36B). Significant differences in volume were also found for both nuclei (averages - small: $468 \pm 35 \mu \mathrm{m}^{3}$, competing: $529 \pm 39 \mu \mathrm{m}^{3}$, dominant: $516 \pm 54 \mu \mathrm{m}^{3} ; \mathrm{p}=0.003, \mathrm{~K}-\mathrm{W}$ ) and cell bodies (averages - small: $1116 \pm 183 \mu \mathrm{m}^{3}$, competing: $1528 \pm 214 \mu \mathrm{m}^{3}$, dominant: $\left.1524 \pm 273 \mu \mathrm{m}^{3} ; p=0.001\right)$. Pairwise comparison of nucleus volumes between developmental stages showed statistical difference between nuclei of cells with small inputs and those with either competing inputs $\left(p=9.51 \times 10^{-4}\right.$, $M-W)$ or a single dominant input $(p=0.012, M-W)$. Similarly, cell body volumes were significantly different only when small category cells were compared with competing $\left(p=6 \times 10^{-4}, M-W\right)$ or dominant $(p=0.003, M-W)$ (Fig. 36C). This indicates one of two potential scenarios. First, postsynaptic maturation in morphology may be highly influenced by the transition of pre-synaptic status from small, exuberant terminals to a more refined innervation pattern in the competitive state. Alternatively, the maturation of the principal cell may be a initiating factor for the pruning of supernumerary inputs. These results agree with those obtained for developmental categories grouped irrespective of age (P2-P9, data not shown), and suggest that changes in intrasomatic polarity are associated with the developmental status and innervation of individual cells rather than the overall developmental age of the animal. 


\section{Terminal Location is Polarized with Respect to Nuclear Position}

To quantitatively assess the placement of growing calyceal terminals, reconstructed cells from P2-P6 were binned in $1 \mu \mathrm{m}$ increments using planes normal to the polarity vector (Fig. 37A). For each bin, the apposed surface area (ASA) — the portion of the terminal in direct apposition to the cell membrane-for the two largest inputs was quantified (Fig. 37B). Nuclear volume within each bin was also recorded for comparison. Cells were grouped either by age (Fig. 38) or developmental state and results were averaged. This analysis was also repeated for P3 alone with similar results (Fig. 39). Our previous work has shown that principal cells have 2-3 competing inputs during development (Holcomb et al., 2013b), and only $29 \%$ of the competing cells reconstructed have 3 competing inputs (7/24 cells, P3-P6). Considering this, we restricted our analysis to the two largest inputs per cell. To determine if terminal placement was significantly different from random, a simulation of random terminal growth was performed, and all terminal curves were compared to the results (Fig. 37B - dotted black line). Significance was determined by comparing the confidence intervals of a modified logistic curve fit to either the simulated curve or the curve data from reconstructed terminals (see Methods). Although multiple curve parameters were considered (lower sill, upper sill, midpoint, slope), we considered the midpoint-the bin at which the input surface area reaches $50 \%$ of its total-to be the most salient factor in determining terminal polarity. Both terminals for small category cells were found to be significantly polarized towards the cytoplasmic pole with respect to their midpoint (Input 1 (I1): bin 6.72, Input 2 (I2): bin 8.64, Simulated (S): bin 11.79; I1 vs S: $p=6.01 \times 10^{-11}, 12$ vs $S: p=0.001 ; n=19$, Fig. 37B - upper left). Comparison between terminals also showed a significant shift in midpoint between I1 and I2, with the second largest terminal consistently less polarized $\left(p=1.95 \times 10^{-5}\right)$. However, terminals at this stage only occupy $1-2$ bins, leading to a high degree of variability in the data. Cells with a single dominant terminal showed significant polarity in the largest terminal $\left(11\right.$ vs $\left.S: p=1.33 \times 10^{-15}\right)$, however the second largest terminal showed no difference in placement from random (I2 vs S: $p=0.611$; Fig. 37B - upper 
right). It is important to note that the average largest terminal size increases from $8.1 \mu \mathrm{m}^{2}$ in cells with small terminals to $254.1 \mu \mathrm{m}^{2}$ in cells with dominant terminals, and that polarity increases significantly in the largest terminal between these two groups $\left(p=3.28 \times 10^{-4}\right)$, indicating that terminals selectively expand on the cytoplasmic pole. Taken together, these results indicate that both initial placement and growth of the calyx is biased towards the cytoplasmic pole.

Competing terminals were also significantly more polar than random placement would dictate (I1: bin 7.68, I2: bin 6.63; I1 vs $S: p=1.74 \times 10^{-10}$, I2 vs $\left.S: p=5.19 \times 10^{-11} ; n=20\right)$, but the difference between these curves showed a much weaker significance ( $p=0.001$; Fig. 37B - upper center). Additionally, comparisons between other curve parameters showed no significant difference between the largest and second largest inputs on these competing cells. To determine if this similarity in terminal polarity is a hallmark of competing cells, each cell was considered individually. The cumulative sum of ASA in bin 10 (i.e., the sum of all ASA on the cytoplasmic pole) for the largest and second largest input were plotted against one another, and k-means clustering was applied for a series of $k$ 's to determine an optimal number of groupings (Fig. 40). This revealed three separate groups of polarity profiles for competing terminals: Type I, where the largest terminal is also the most polarized, and the second largest is not significantly different from random; Type II, where the second largest terminal is the most polarized, and the largest is random; and Type III, where both terminals are highly and almost equally polarized (Fig. 37B - lower). One cell that was identified by the k-means clustering as being in the Type II group appeared to be an outlier (Fig. 40). The ratio of input 1 to input 2 for this cell $(0.74)$ was much closer to the average Type 3 ratio (0.93) than that of Type 2 (0.39). However, both terminals of this cell appeared to be anti-polar-that is, located on the nuclear pole rather than the cytoplasmic pole. As previous work by Sätzler et al. clearly demonstrated the presence of an anti-polar calyx on an MNTB cell at postnatal day 9 in the rat (Sätzler et al., 2002), we decided to qualitatively explore the prevalence of anti-polar calyces in our older ages (P6, P9). Of the 43 
cells at P6 examined (15 qualitatively, 28 quantitatively), 84\% possessed calyces located on the cytoplasmic pole (36/43), and only 5\% (2/43) possessed anti-polar calyces. The remaining cells either had no clear calyx $(9 \%, 4 / 43)$ or were equally distributed between both poles $(2 \%, 1 / 43)$. As the calyx matures, terminal morphology changes from a solid, spoon-like shape into a highly fenestrated structure (Hoffpauir et al., 2006; Ford et al., 2009). Due to this morphological change, qualitative analysis of polarity at P9 was complicated and an accurate qualitative assessment of polarity at P30 was impossible. A total of 66 cells at P9 were examined (55 qualitatively, 11 quantitatively), with $26 \%$ (17/66) of these having an indeterminate result due to fenestration and an additional $12 \%(8 / 66)$ having no clear calyx. Of the remaining cells, $53 \%$ (35/66) had a clearly polar calyx, and only $9 \%$ (6/66) possessed an anti-polar calyx (Fig. 41). This suggests that, while anti-polar calyces are present in the MNTB, the population of cells possessing an anti-polar calyx is small. Overall, these results suggest that terminal placement and growth is predisposed toward the cytoplasmic pole of the MNTB principal cell, and that polarized placement may be one of the competitive mechanisms used in determining terminal dominance.

\section{Membrane is Added Asymmetrically During Principal Cell Growth}

Our results indicate both a polarized expansion of the postsynaptic cell away from the nuclear location and biased growth of terminals on the cytoplasmic pole. This suggests that membrane addition during principal cell growth is also a polarized process. Principal cells also develop multiple thin processes that appear to increase in number from P3 to P6, but are less prevalent by P9 (Sätzler et al., 2002; Holcomb et al., 2013b). These processes come in two types: short "somatic spicules", many of which project into growing terminals, and long, thin projections devoid of organelles that do not appear to be innervated (Fig. 42A - left). To explore membrane distribution during development in MNTB principal cells, surface area was measured for each reconstructed cell. Each cell was also simplified using a screened Poisson reconstruction which 
maintained general surface topography and cell volume but removed processes (Kazhdan and Hoppe, 2013) to quantify membrane on somatic processes (Fig. 42A - right). A significant increase in surface area occurred between P2 and P4, during which time the average surface area of cells more than doubled $\left(708.1 \mu \mathrm{m}^{2}\right.$ at $\mathrm{P} 2,1518.3 \mu \mathrm{m}^{2}$ at $\mathrm{P} 4 ; \mathrm{p}=1.88 \times 10^{-6}, \mathrm{M}-\mathrm{W}$; Fig. 43A). The surface area of somatic processes_calculated as the difference in surface areas between the original cell reconstruction and the Poisson reconstruction-increased from $16 \%$ of the average cell surface area at P2 to $50 \%$ at P4; increase in somatic process area accounted for $80 \%$ of the membrane expansion between P2 and P4, but only represented $1.4 \%$ of the P4 cell volume (Fig. 42B). The cell membrane-discounting somatic processes-also undergoes significant expansion over this period (average surface area of Poisson reconstructions: $596.5 \mu \mathrm{m}^{2}$ at $\mathrm{P} 2,756.2 \mu \mathrm{m}^{2}$ at $\mathrm{P} 4, \mathrm{p}=3.21 \times 10^{-5}$ ), potentially providing new surface upon which nascent terminals can grow (Fig. 43A). Although no significant change in total surface area of cells (including spicules) was found after P4, a significant decrease (P4-P6) and subsequent increase (P6-P9) in surface area of Poisson reconstructions was evident. This may represent a reorganization of membrane between the main cell body and somatic processes, as these processes qualitatively increase in number and then mostly disappear during this timeframe.

Presynaptic activity has been shown to be necessary to maintain principal cell soma size (Weatherstone et al., 2016) and modulation of synaptic activity can increase or decrease the insertion of membrane into the subsynaptic reticulum at the Drosophila neuromuscular junction (Teodoro et al., 2013). As many somatic spicules can be found embedded in the growing calyx (Sätzler et al., 2002; Holcomb et al., 2013b), it is possible that calyx activity may induce growth of somatic spicules. If this is the case, somatic spicules should have a polarized distribution similar to developing terminals. To this end, we examined whether a difference in somatic spicules was present between the nuclear and cytoplasmic poles. Cells were bisected through the center of mass as for previous calculations, and surface area was computed for the 
cytoplasmic and nuclear poles for both spiculated and Poisson reconstructions. The surface area of the cytoplasmic pole for spiculated P4 and P6 cells was significantly larger than their nuclear pole counterparts (Fig. 42C; P4: $834 \pm 191 \mu \mathrm{m}^{2}$ for CP versus $684 \pm 103 \mu \mathrm{m}^{2}$ for NP, $p=0.003, M-W ; P 6: 876 \pm 292 \mu m^{2}$ for $C P$ versus $627 \pm 122 \mu m^{2}$ for NP, $\left.p=0.006, M-W\right)$. However, this significance was abolished in the Poisson reconstructed cells, suggesting that the difference was confined to the somatic spicules. This asymmetry in somatic spicules may be a result either of activity-dependent induction of postsynaptic growth or the natural development of the principal cell irrespective of innervation state. To differentiate between these two cases, the surface area for each pole was assessed for the three developmental states at P3 (Fig. 42D). No significant difference in surface area was found between NP and CP in any of the three developmental states, either for spiculated or Poisson reconstructed cells. Comparison of NP and CP between developmental states, however, revealed a significant increase in surface area on both poles for competing and dominant cells compared to cells with only small inputs (CP: $392 \pm 83 \mu m^{2}$ for small versus $560 \pm 86 \mu m^{2}$ for competing $\left(p=6 \times 10^{-4}, M-W\right)$ or $628 \pm 160 \mu m^{2}$ for dominant $(p=0.002, M-W)$; NP: $403 \pm 90 \mu m^{2}$ for small versus $543 \pm 87 \mu m^{2}$ for competing $(p=0.021$, $M-W)$ or $533 \pm 91 \mu m^{2}$ for dominant $\left.(p=0.022, M-W)\right)$. These results were similar for the whole cell case as well (Fig. 43B). Unlike the comparison of CP and NP surface area by postnatal day, the significant difference between developmental states was not abolished in the Poisson reconstructed cells; the remaining surface area increase most likely represents the membrane added directly to the cell body that results in an increase in overall principal cell size, similar to the expansion seen in whole Poisson reconstructed cells compared across postnatal days. Taken together, these results suggest a bias in membrane addition toward the cytoplasmic pole that is potentially induced by terminal growth and dominated by extension of somatic spicules.

The division of the cytoplasmic and nuclear poles of the principal cell was performed by bisecting the cell with a plane passing through the cell center. However, as the cell matures the 
center of the cell changes position. If the functional polarity of the cell is established early in development, the division of cytoplasmic and nuclear hemispheres may be more asymmetric than we have predicted at later ages (P4-P9). To explore how this may affect our measurements, the surface areas for cells from P3-P9 were remeasured using the average placement of the bisecting plane at $\mathrm{P} 2(1.83 \mu \mathrm{m}$ from the nucleus center along the polarity vector). As expected, an increase in cytoplasmic pole surface area and concomitant decrease in nuclear pole surface area occurred, with stronger effects at later ages (Fig. 44; change in CP: $11.9 \%$ at $\mathrm{P} 3,17.6 \%$ at $\mathrm{P} 4,16 \%$ at $\mathrm{P} 6$, and $21.5 \%$ at $\mathrm{P} 9)$. These changes would not affect our conclusions; if anything, an increase in cytoplasmic pole surface area would only increase the polarity of calyceal terminals and principal cell structures.

\section{Glial Apposition Restricts Innervation from the Nuclear Pole}

Extrinsic factors may also influence the placement and growth potential of terminals during calyx formation. One such factor is glial ensheathment of the cell body; glial ensheathment has been shown to fluctuate based on hormonal and environmental cues and affect the availability of somatic territory for innervation (Theodosis et al., 2008). Glial vellous processes interact with MNTB principal cells and calyces as early as P0, positioning them to potentially influence synaptic connections (Hoffpauir et al., 2006). To determine the potential influence of glial ensheathment on placement and expansion of the growing calyx, glial apposed surface areas (gASA) were reconstructed for ten cells each at P2-P3 and five cells each at P4 and P6 (Fig. 45A-A"). As previously, cells were subdivided into cytoplasmic and nuclear hemispheres, and percentage of glial coverage was calculated for each hemisphere (Fig. 45B). Glial processes were rarely found on somatic spicules, so for an unbiased comparison of glial coverage percentages the Poisson reconstructed cells for P3-P6 were used for these calculations. In addition, as other extracellular structures may limit available surface area, the amount of cellcell contact (either somatosomatic or somatodendritic) and somatic surface area covered by 
fiber fascicles was also quantified. No significant change in gASA on either the nuclear pole or cytoplasmic pole was found when each hemisphere was compared across ages, though the change in nuclear pole gASA across ages approached significance (P2-P6, $p=0.078, K-W)$. However, when hemispheres were compared within ages, a significant difference in nuclear pole to cytoplasmic pole gASA was evident at P4 (average glial surface area of $232.7 \mu \mathrm{m}^{2}$ for $\mathrm{NP}$ versus $174 \mu \mathrm{m}^{2}$ for $\left.\mathrm{CP}, \mathrm{p}=0.038, \mathrm{M}-\mathrm{W}\right)$. Additionally, the percent coverage of the $\mathrm{P} 4$ nuclear pole was significantly greater than that of the cytoplasmic pole (Fig. 45C, $63 \pm 13 \%$ for NP versus $44 \pm 9 \%$ for $C P, p=0.038, M-W)$. This shift of glial coverage from P3 to P4 could represent either a retraction of glial processes to expose more surface area for innervation or a growth of cell membrane without concomitant growth of associated glia. If glial processes were removed from the cell body, the total gASA should decrease as development proceeds, however no significant difference in gASA was found (Fig. 45D). As a large amount of somatic surface area is added between P3 and P4 $\left(666.1 \mu \mathrm{m}^{2}\right.$ at P3 versus $756.2 \mu \mathrm{m}^{2}$ at P4, Poisson reconstructions, $\mathrm{p}=0.007$, $\mathrm{M}-\mathrm{W}$ ) and this addition of surface area is biased towards the cytoplasmic pole (Fig. 42C), it is reasonable to surmise that this is the source of the apparent redistribution of gASA. This is particularly apparent when glial coverage is considered including spicule surface area: total cell surface covered by glial processes drops from $45 \%$ at P2 to $25 \%$ at P6, driven mainly by a drop in cytoplasmic pole coverage from $46 \%$ at P2 to $20 \%$ at P6 (Fig. 46).

\section{Principal Cell Axon and Dendrite Placement is Oppositional and Biased Toward the Cytoplasmic Pole}

Prior to the appearance of axosomatic terminals (P0-P1), Hoffpauir et al. found that principal cell innervation was largely confined to the dendrites of these cells (Hoffpauir et al., 2006). To determine whether polarized placement of the dendrites may influence positioning of axosomatic terminals, the angle of the center of the proximal dendrite with respect to the polarity vector was assessed for P2-P9 (Fig. 47A). At all ages, greater than $68 \%$ of dendrites originated 
on the cytoplasmic pole (Fig. 47B; $75 \%$ at $\mathrm{P} 2,69 \%$ at $\mathrm{P} 3,69 \%$ at $\mathrm{P} 4,70 \%$ at $\mathrm{P} 6$, and $77 \%$ at P9). The average angle of dendrites with respect to the polarity vector did not change significantly over the course of development $\left(72.5 \pm 27^{\circ}\right.$ at $\mathrm{P} 2,67.3 \pm 38^{\circ}$ at $\mathrm{P} 3,73 \pm 43^{\circ}$ at $\mathrm{P} 4$, $70.5 \pm 44^{\circ}$ at $\mathrm{P} 6$, and $76.9 \pm 23^{\circ}$ at $\left.\mathrm{P} 9 ; \mathrm{p}=0.787, \mathrm{~K}-\mathrm{W}\right)$. The general stability of dendrite angles was also apparent when developmental stage was considered (average angles: $69.4 \pm 30^{\circ}$ for small, $71.8 \pm 41^{\circ}$ for competing, and $70.2 \pm 42^{\circ}$ for dominant; $p=0.868, \mathrm{~K}-\mathrm{W}$ ) and confirmed by confining our measurements to only P3 $(p=0.873, K-W)$. Cells at P2 have a more fusiform appearance compared to later time points; no cell at P2 possessed dendrites at $<20^{\circ}$ from the polarity axis, compared to $\sim 10 \%$ of dendrites at $<20^{\circ}$ from P3-P9 (Fig. $47 \mathrm{C}$ ). These results show a tendency for principal cell dendrites to be located on the cytoplasmic pole.

Many cell types in the central nervous system, such as cerebellar granule cells and pyramidal cells in the cerebral cortex and hippocampus, define their dendrites and axon during migration from the diametrically opposed leading and trailing process (for a review, see Barnes and Polleux, 2009). Though the axons and dendrites of principal cells are already well defined by $\mathrm{P} 2$, the presence of dendrites and axons diametrically opposed to one another may suggest similar developmental processes at work in MNTB development and possibly indicate that MNTB cell polarity is established embryonically. Axon position with respect to the polarity axis was determined for each cell in the same manner as for dendrites. On average, axons appear to reside equatorially with regards to the polarity axis of the cell early in development, though there is a high degree of variability in placement (Fig. 47D; average angles: $91.5 \pm 29^{\circ}$ at $P 2,91.6 \pm 43^{\circ}$ at $\mathrm{P} 3,65 \pm 41^{\circ}$ at $\mathrm{P} 4,75.8 \pm 43^{\circ}$ at $\mathrm{P} 6$, and $77.7 \pm 40^{\circ}$ at $\left.\mathrm{P} 9 ; \mathrm{p}=0.242, \mathrm{~K}-\mathrm{W}\right)$. More than half of the axons at P2 emerge from a dendrite (Fig. 47E), and this number decreases until P9 when this phenomenon is no longer apparent (Fig. 47F; percentage of axons emerging from dendrites: $62 \%$ at $\mathrm{P} 2,17 \%$ at $\mathrm{P} 3,29 \%$ at $\mathrm{P} 4,8 \%$ at $\mathrm{P} 6,0 \%$ at $\mathrm{P} 9)$. This suggests that dendrites with associated axons may be selectively pruned during development. Axodendritic angle was then 
determined by measuring the angle with respect to the cell center from the base of the axon to the average location of all dendrites. As the emergence of an axon from a dendrite is a transient phenomenon, those dendrites were excluded from this assessment. Only $21 \%$ of cells possessed an axon within $90^{\circ}$ of the average dendrite location (16/76 cells), and all ages measured had an average axodendritic angle $>100^{\circ}$ (Fig. 47G; average axodendritic angles: $136.4 \pm 28^{\circ}$ at $P 2,105.9 \pm 50^{\circ}$ at $P 3,119.5 \pm 33.5^{\circ}$ at $P 4,113.5 \pm 29^{\circ}$ at $P 6$, and $114 \pm 17^{\circ}$ at $P 9$ ). No significant change in axodendritic angle was found across ages $(p=0.25, K-W)$, or when developmental stage was considered at P3 $(p=0.638, K-W)$. Though not diametrically opposed, these results do suggest that dendrites and axons are segregated to opposing areas of the cell surface following the removal of axon-associated dendrites. Intriguingly, both dendrites and axons appear to have a slight bias towards the cytoplasmic pole despite being located on opposite sides of the cell, resulting in both being located near the "equator" between the cytoplasmic and nuclear poles. As these cells have a fusiform shape early in development, these later axodendritic positions may be a slightly relaxed holdover from this early morphology.

\section{Calycigenic Axons Align with Dendrites Prior to Innervating the Principal Cell Soma}

The presence of innervated dendrites with a positional bias for the cytoplasmic pole as early as P2 suggests that dendrites may serve as guides for axons toward the soma, and that axodendritic association may increase the likelihood of an axon attaining a favorable position for forming a large terminal. To evaluate this, a qualitative assessment of GBC axon-PC dendrite association was performed for the two largest terminals $(11,12)$ of cells with either competing or single dominant terminals (16 cells each at P3-P4, 10 cells at P6). Terminal axons were divided into three categories based on their dendrite association: the axon contacts and follows the principal cell dendrite to the soma ("tracks", Fig. 48C,E), the axon touches down at the base of a dendrite without tracking along it ("base contact", Fig. 48D,H), and the axon is not associated with any dendrite ("no association", Fig. 48F,G). At P3, the axons of both large terminals were 
highly associated with dendrites (Fig. 48A; "tracks": $88 \%$ for I1, 56\% for I2; "base contact": $0 \%$ for I1, 22\% for I2; "no association": $12 \%$ for $\mathrm{I1}, 22 \%$ for I2). A similar result is evident at P4, though the distribution of axons for I1 and I2 are inverted compared to P3 ("tracks": $56 \%$ for I1, $75 \%$ for I2; "base contact": $13 \%$ for both I1 and I2; "no association": $31 \%$ for I1, $12 \%$ for I2). However, by P6 the dendritic association with axons from both inputs decreased ("tracks": $50 \%$ for I1, $29 \%$ for I2; "no association": $50 \%$ for $11,71 \%$ for I2). One intriguing possibility for this is a reduction in the number of dendrites following successful capture of calycigenic axons. To examine this possibility, the average number of dendrites per cell for each age was calculated (Fig. 48B). These measurements revealed two phases of dendrite pruning, one from P2-P3 (5.23 \pm 1.2 to $3.59 \pm 1.1$ dendrites, $p=2 \times 10^{-4}, M-W$ ) and a second from P4-P6 (4.35 \pm 1.4 to $2.5 \pm 0.5$ dendrites, $\left.p=9.98 \times 10^{-5}, M-W\right)$. The timing of these dendritic reductions corresponds well to the transition in phases of competition for terminals described previously (Holcomb et al., 2013b). These reduction phases have been termed the "early" phase (P2 and earlier, 5+ dendrites), "primary" phase (P3-P4, 3-4 dendrites), and "secondary" phase (P6-P9, 2 or fewer dendrites). Based on these findings, assessment of axon-dendrite association was performed for both P3 cells alone and pooled P3-P4 cells to avoid the confound of additional dendritic pruning at P6 (Fig. 49). Terminals in competition showed a high propensity to track dendrites to the soma, both in the P3-only and pooled P3-P4 cases with no significant difference between I1 and I2 (P3-only "tracks": $78 \%$ for $11,71 \%$ for I2, p=0.771, Chi-squared; P3-P4 pooled "tracks": 85\% for I1, $80 \%$ for I2, $\mathrm{p}=0.772$, Chi-squared). In contrast, a significant difference in axodendritic association was evident between I1 and I2 in the dominant case (P3-only "tracks": 100\% for I1, $29 \%$ for I2, p=0.005, Chi-squared; P3-P4 pooled "tracks": $71 \%$ for I1, 28\% for I2, p=0.011, Chisquared). These results demonstrate both a significant association between calyx-forming axons and PC dendrites and a multi-stage dendritic pruning not described before for MNTB PCs. 


\section{Discussion}

The results of this investigation demonstrate a novel polarity in development of both the calyx of Held and its postsynaptic target, the principal cell of the MNTB. Developing principal cells are characterized by eccentrically placed nuclei that establish an "intrasomatic polarity" that persists through young adulthood (P30). This polarity appears to define a unique territory opposite of the nuclear location that is amenable to growth of the calyx, is enriched in dendrites, and is selectively enlarged as the principal cell matures to create glia-free surface area for innervation. To our knowledge, this is the first report of a polarity program in the coordinated pre- and postsynaptic development of a non-laminar brain region. Additionally, our findings have codified a progression of dendritic pruning in the maturation of principal cells that may influence and be influenced by the developmental state of the cell.

\section{Experimental considerations}

Although the process of segmentation and reconstruction of neurons and associated structures from SBEM is a time-intensive undertaking, the resulting 3D models provide a more complete and structurally quantitative view of the developing auditory system than has previously been possible with light microscopy or traditional electron microscopy. Several limitations to this technique are worthy of note, however. The number of cells readily available for analysis is necessarily lower in SBEM due to restrictions on tissue sample size and time constraints for segmentation. As semi-automated and automated segmentation techniques become more reliable and less computationally intensive, reconstruction of 3D models will prove less of a bottleneck to ultrastructural analysis. The 24-hour separation in our time points and static nature of SBEM also present constraints to our study. For instance, astroglial processes can be remodeled on a time scale of minutes-a dynamic process that this technique is not capable of capturing (Hirrlinger et al., 2004). The stability of gASA measurements across ages, however, suggests that while transient changes in glial coverage may occur, the overall glial coverage of 
principal cells remains constant. Dendrite pruning can also occur rapidly ( $\sim 5$ hours in Drosophila sensory neurons, Kanamori et al., 2015) necessitating a technique capable of capturing rapid changes in dendrite morphology in a living system. These changes in dendrite morphology are particularly important in the assessment of axodendritic association. In early MNTB development (P2-P3), dendrites frequently branch proximally, while these bifurcations are less prevalent at later time points (Fig. 47B, compare dendrites at P2-P4 to those at P6-P9). Axons that follow a dendritic branch that is pruned may appear later to only have associated with the dendrite base; Fig. 48D represents a possible example of this. Similarly, an axon that tracks down a dendrite that is removed completely prior to tissue fixation and imaging may be misidentified as never having been associated with a dendrite. Therefore, our measurement of axodendritic association is most likely an underestimation, particularly at P6. Despite these limitations, the advantages of nanoscale resolution and excellent alignment of images in SBEM has allowed us to assess the morphology of the developing MNTB with a degree of precision significantly better than other imaging techniques.

\section{Defining Polarity in Neuronal Systems}

Polarity based on the arrangement of intracellular organelles (“intrasomatic polarity") was used in this study to operationally define compartments of the MNTB principal cell. Neuronal polarity has classically been defined either macroscopically through position of the axon and dendrites or at the protein level through asymmetric localization of canonical polarity proteins. For instance, Calderon de Anda et al. defined polarity of pyramidal neurons based on the appearance of the first two diametrically opposed neurites, one of which becomes the axon (Calderon de Anda et al., 2008). In inner hair cells, asymmetric trafficking of proteins such Vangl2, Scribble1, and Frizzled (Fz3) orient these cells in a uniform fashion to guarantee proper stereocilia function (Montcouquiol et al., 2006). Importantly, multiple polarity proteins have been implicated in the formation of synapses (Wiggin et al., 2005). For instance, Par3 is recruited to 
developing synapses where it participates in a signaling pathway responsible for cytoskeletal changes vital to synapse formation (Duman et al., 2013). Par3 has also been shown to mediate another vital aspect of cellular polarity: localized membrane addition through the exocyst complex, a multi-protein complex involved in vesicle docking for exocytosis (Lipschutz and Mostov, 2002; Lalli, 2009; Das et al., 2014). Additionally, activity-dependent expansion of the sarcoplasmic reticulum at the neuromuscular junction requires both RalA and the exocyst complex (Teodoro et al., 2013). These findings present an intriguing narrative that may explain many of the observations in our study. Exocyst function has been explored in the calyx by overexpression of a dominant negative version of Exo70, an exocyst subunit, through viral injection into the ventral cochlear nucleus. While no functional phenotype was found, a significant disruption in structure and decrease in calyx size were evident at P13. However, the timeline of viral expression in these cells is unclear, calyces were only assessed at P13 and later, and changes in the MNTB principal cells were not assessed (Schwenger and Kuner, 2010). Further research is necessary to determine if polarity-associated proteins are asymmetrically trafficked to the cytoplasmic pole of the principal cell. In particular, if polarity proteins known to promote synapse formation are found to aggregate in the CP prior to somatic innervation, it would represent a pre-patterning of the postsynaptic cell in preparation for terminal formation similar to that observed in the neuromuscular junction (Jing et al., 2009).

\section{Polarity in Terminal Formation: Similarities to the Climbing Fiber:Purkinje Cell System}

Due to its rapid development, exceptional size, and well-defined one-to-one partnership with the MNTB principal cell, the calyx of Held represents an excellent model system for studying terminal development and competition. Given the results of this investigation, determining if the morphological characteristics and innervation constraints found in calyx and principal cell development are translatable to other similar systems is an important avenue of future research. Some evidence already exists that hints at parallelism between calyx of Held:MNTB 
development and an analogous system, the connection between climbing fibers and Purkinje cells (CF:PC) in the cerebellum. The CF:PC connection undergoes a multi-stage developmental process that refines the connection from an exuberant, multi-innervated state to a monoinnervated endpoint as in the MNTB (for a comprehensive review, see Watanabe and Kano, 2011). From E19-P5, CFs innervate and track along Purkinje cell dendrites to reach the cell body (the "creeper" stage), similar to the axodendritic association shown here in the MNTB (Morara et al., 2001). Concurrent with this, PCs undergo changes in dendritic morphology, beginning with a simple fusiform shape at E19, increasing in dendrite number and complexity at P1 (“complex fusiform"), and then beginning to retract until their complete removal at P6 (Armengol and Sotelo, 1991). Intriguingly, this process mirrors well the multi-stage dendritic pruning seen in our principal cell results. Following this (P5-P9), the CF terminals densely innervate the Purkinje cell soma in the "pericellular nest" stage (Hashimoto et al., 2009). During this stage, a single CF increases in both the number of terminals on the Purkinje cell surface and the strength of these terminals, so it is an intriguing possibility that CFs with more apical terminals may be selectively strengthened (Kano and Hashimoto, 2012). The next developmental stage ("capuchon" stage, P9-P12) involves multiple CFs translocating to apical pole where the primary dendrite is located. In the final developmental stage ("dendritic stage", $\sim P 12)$ the strongest CF — the fiber capable of inducing the largest EPSC in the Purkinje cell — begins innervating the apical dendrite, while weaker CFs are excluded from the dendrite and eventually removed (Hashimoto et al., 2009). The pruning of "losing" inputs is accomplished in two stages: an early stage that lasts until P10 and is independent of innervation of Purkinje cell by parallel fibers (PFs), and a second stage that requires PF activation of metabotropic glutamate receptors to eliminate supernumerary CFs. Though longer in time course, these two phases of synapse elimination are similar to the pruning of inputs in calyx development (Holcomb et al., 2013b). Most importantly for comparison, Purkinje cells appear to have an eccentric nucleus at early developmental ages based on both light and electron microscopy 
(Armengol and Sotelo, 1991 - Fig. 8A, P6; Morara et al., 2001 - Fig. 1D, P6, Fig. 2A, P0; Dunn et al., 1998 - mouse in vitro). A comprehensive 3D reconstruction of Purkinje cells and associated terminals across the salient time points detailed here is necessary to determine if the implied correlations to our findings are accurate. If these similarities are verified, it strengthens the possibility that the findings in this study may be generalized to other developing neural systems.

\section{Functional Consequences of Nuclear Eccentricity}

Despite the vital role nuclear positioning plays in nervous system development, the relationship between nuclear position and neuronal innervation has received very little attention.

Quantitatively, we have demonstrated that principal cell nuclei are eccentrically positioned as early as P2; qualitatively, this eccentricity can be seen in images of principal cells as early as P0 (Hoffpauir et al., 2006). The function of an eccentric nucleus is still unclear, however. One potential explanation involves optimization of nuclear readout of synaptic activity. Bollmann et al. showed that voltage gated calcium channels on the principal cell of P8-P10 rats are located opposite the calyx and near the nucleus, possibly positioning calcium influx to influence the transcriptional profile of the developing cell (Bollmann et al., 1998). Synaptically activated internal calcium release causes calcium waves in immature pyramidal cells (1-3 weeks) have been shown to increase phosphorylation of cAMP Response Element-Binding protein (CREB), an important transcription factor present in the nucleus (Yeckel et al., 2008). In addition to their eccentric location, principal cell nuclei are also infolded in morphology based on qualitative assessments (Holcomb, unpublished data). Calcium flux through the nucleus is significantly affected by its shape; while a spherical nucleus acts as an integrator for calcium fluxuations, nuclear infolds create microdomains capable of detecting high frequency calcium waves (Wittmann et al., 2009; Queisser et al., 2011). The infolding of the principal cell nucleus and eccentric location may allow the rate of calyx firing to evoke calcium waves and elicit changes in 
transcriptional regulation, both through phosphorylation of nuclear-resident transcription factors and chromatin remodeling (Queisser et al., 2011). A secondary consequence of nuclear eccentricity is a polarized concentration of organelles-particularly protein translation machinery - to the region opposite the nucleus. This creates a directionality to transcription and translation that may influence protein trafficking necessary for supporting terminal growth on the cell surface opposite the nucleus. Further work is necessary to confirm these theories and to determine the developmental consequences of losing nuclear eccentricity.

\section{Glial Coverage and Principal Cell Innervation}

Structural plasticity in astrocytic processes has varied and system-specific consequences for neuronal function. For instance, glial retraction in the magnocellular neurons of the supraoptic nucleus resulting from multiple stimuli (dehydration, lactation, restraint stress, etc.) has been shown to increase cell-cell contact and provide the axons of these cells greater contact area with capillaries. In the arcuate nucleus of mice, the amount of neuron surface coverage by glia corresponds to fluctuations in sex hormones (Theodosis et al., 2008). Similarly, our measurements show that thin vellous glial processes consistently cover $50 \%$ or more of the somatic surface of principal cells. Although the distribution of glial processes changes significantly during the period of explosive growth for both the calyx and principal cell (P3-P4), the total surface area of glial apposition did not change significantly, suggesting that cell growth—particularly from somatic spicules (Fig. 46)—and not glial retraction mediates the increase in available surface area for innervation. Glial coverage is also apparent surrounding growing terminals and interposing between the terminal and principal cell (Hoffpauir et al., 2006; Reyes-Haro et al., 2010; Holcomb et al., 2013b). Astrocytes apposed to calyces experience slow inward currents upon terminal activation, demonstrating an electrophysiological coupling between these elements (Reyes-Haro et al., 2010). Similar associations have been found for Bergmann glia and climbing fibers in the cerebellum, though while the response in Bergmann 
glia is AMPA-dependent, the astrocytic response in the MNTB is independent of AMPA receptors. Intriguingly, when Bergmann glia AMPA receptor subunit composition was altered, glial processes retracted from terminals and Purkinje cell dendrites became innervated by multiple climbing fibers (lino et al., 2001). Although time constraints did not allow for quantification of glial coverage on inputs as well as principal cells, this analysis should be a priority in future work. Additionally, manipulation of astrocytes in the MNTB to abolish their sensitivity to presynaptic activation should be undertaken to determine if astrocytic wrapping of terminals may play a vital role in competition.

\section{Dendritic Capture of Calycigenic Axons}

Dendritic capture of axons for somatic innervation has not previously been described. Our results suggest that axodendritic association is a prevalent phenomenon in development of the calyx of Held; many axons appear to use dendrites as "highways" to track to the principal cell soma, and axodendritic association may play a role in terminal competition. If axodendritic association influences the competitive process, one would expect competitive terminals to both be associated with a dendrite while cells with a single dominant terminal would have only the largest input dendritically associated. The decrease in association between the axon of the second largest input and a PC dendrite for cells with dominant inputs in our data indicates that this may be the case. However, if somatic innervation induces dendritic pruning, this may lead to an underestimation of axon-dendrite association in this case. Further analysis of dendritic morphology and innervation is currently being undertaken to provide a clearer picture of this phenomenon.

\section{Dendrite Pruning}

During development, both axons and dendrites are refined to create accurate circuit connections. We demonstrate here for the first time that the pruning of principal cell dendrites is a multi-stage process that corresponds temporally to removal of supernumerary inputs to a 
monoinnervated state. Additionally, a greater propensity for axodendritic association in competing versus single dominant cases suggests that dendrites may contribute to what is most likely a multi-faceted set of competitive mechanisms that will define the calyx. Many outstanding questions remain in the removal of principal cell dendrites, chiefly the mechanism by which the dendrites are removed. In the chick nucleus magnocellularis, dendrites are removed through retraction following downregulation of the neurotrophin receptor Tropomyosin Receptor Kinase B (TrkB) (Schecterson et al., 2012). Dendrite removal in Drosophila sensory neurons is accomplished by endocytic thinning of dendrites modulated by calcium transients and leading to dendritic pruning (Kanamori et al., 2015). External factors may also play a role in dendrite removal; organotypic cultures of E13 chick nucleus laminaris exposed to astrocyte conditioned media from more mature astrocytes lost substantially more dendrites than control cultures (Cramer and Rubel, 2016). Glial processes can frequently be seen in apposition to principal cell dendrites in SBEM images, making them well positioned to mediate dendritic pruning (Holcomb et al., 2013b). The exact mechanism for dendritic removal in MNTB development remains to be described, and the consequence of early loss of dendrites on principal cell innervation should be studied in detail.

In conclusion, this study defines several novel morphological aspects of the development of the calyx of Held:principal cell connection and provides promising new avenues of research to determine competitive mechanisms driving the selection of the terminal that will become the calyx of Held. The rich quantitative information provided by 3D reconstructions of developing MNTB cells and calyceal terminals fills a gap in our knowledge of morphological transitions that occur during the formation and maturation of the calyx of Held. Although remarked upon previously (Bollmann et al., 1998; Hoffpauir et al., 2006), the results of positional quantification of terminal growth in opposition to nuclear location in this study creates constraints on terminal growth that must be considered in future studies. 


\section{Chapter 4: Discussion and Future Directions}

\section{Competition in the Developing MNTB}

Competition is a necessary mechanism for removing supernumerary inputs in the developing nervous system and is accomplished in multiple ways depending on the system. In the climbing fiber:Purkinje cell connection, multiple climbing fibers innervate the Purkinje cell but only one strengthens its ability to evoke a postsynaptic response, ascend the apical dendrite, and remain on the cell (Hashimoto et al., 2009). Competition at the neuromuscular junction involves multiple terminals vying for territory on the motor end plate, with a weaker terminal potentially winning out in a process called 'flip-flop' (Walsh and Lichtman, 2003). The projection of the retinal ganglion cells to the lateral geniculate nucleus is refined through spontaneous activity to ensure the correct mapping of ocular information (Hong and Chen, 2011). Although it has been shown that multiple excitatory terminals innervate the principal cells of the MNTB early in development, competition has generally been discounted as a mechanism for refinement of this connection (Kandler and Friauf, 1993; Hoffpauir et al., 2006, 2010; Rodríguez-Contreras et al., 2006). The research presented here provides direct ultrastructural evidence of multiple large terminals innervating a single postsynaptic target in the developing MNTB during a period of explosive growth of the calyx (P3-P4). Importantly, based on our two-photon data (Fig. 28) these terminals most likely originate from different globular bushy cell axons, making them distinct units that must undergo a selection process to determine which will eventually become the calyx of Held. Terminals of at least $50 \%$ of the principal cells in the MNTB appear to undergo competition, though given the 24-hour time difference in our samples this is likely an underestimate. These results also demonstrated that terminal selection is not a function of the age of the animal. As development of the calyx is known to be tonotopically graded, with the medial portion of the MNTB developing before the lateral, we confined our SBEM data sets to the medial portion to attempt to obviate this confound (Ford et al., 2009). Despite this, cells were found at P3-P6 at 
multiple stages of development, which suggests that development of the $\mathrm{CH}$ :MNTB connection is directed at the level of the individual cell. A particularly surprising finding was that $23 \%$ of cells examined at P6 remained in competition, a time course longer than the 48-72 hours previously believed necessary to affect monoinnervation in the MNTB (Hoffpauir et al., 2006). However, half of these cells had a largest terminal measuring only $50 \mu \mathrm{m}^{2}$ in apposed surface area, and one additional cell had no terminals that surpassed the threshold of $25 \mu \mathrm{m}^{2}$ to be considered in the competing or dominant categories. These cells may represent a small subpopulation of nonprincipal cells that have been described previously, though more characterization is necessary to confirm this (Morest, 1968b; Banks and Smith, 1992).

The most likely driving force behind the pruning of competing inputs in the MNTB is a refinement of the tonotopic map to ensure proper frequency matching (Kuwabara et al., 1991). Although Rodriguez-Contreras et al. showed that multiple calyces from the same axon innervate principal cells with very similar mediolateral locations, these measurements were performed in P12-P18 rats, after the onset of hearing and significantly after growth and competition of terminals forming the calyx of Held. Ford et al. demonstrated a loss of tonotopically graded development of fenestration in the absence of spontaneous activity from the cochlea, suggesting that aspects of calyceal development rely on frequency-specific signals. However, these experiments were again performed in P10 and later animals, missing the key developmental window for competition and pruning. Our results show that a single largediameter axon (assumed to be a globular bushy cell axon) can innervate 20 or more cells within our tissue volume at P2 (see Chapter 2 results); if this exuberant branching is found throughout the MNTB, tonotopic refinement is a necessary consequence. One of the current drawbacks of the SBEM technique is a small tissue sample field of view $(\sim 100-150 \mu \mathrm{m}$ in mediolateral and dorsoventral dimensions for our tissue volumes), so we were unable to track calycigenic axons into more lateral areas of the MNTB to determine if they formed calyces outside of the primary 
isofrequency lamina within which they were found. Future experiments must focus on determining the full extents of MNTB innervation by a single globular bushy cell axon to discern whether tonotopic refinement is necessary in $\mathrm{CH}$ :MNTB development.

As competition is mediated at the level of the individual principal cell, local mechanisms for selecting a "winning" input must be considered. Based upon our results, direct physical contact does not appear to influence competition, as competing terminals occupy unique areas of the postsynaptic cell without overlap. However, as these reconstructions represent a single snapshot of the cellular ultrastructure, retraction and expansion causing terminals to come into contact cannot be discounted. Purves and Lichtman have suggested that terminals may compete for a "trophic" factor, suggesting interplay between the pre- and postsynaptic cell may be necessary to affect terminal refinement (Purves and Lichtman, 1980). Although protein expression cannot be assessed in our SBEM image volumes, we were able to measure local morphological factors such as changes in pre- and post-synaptic structure that may correlate with differences in protein expression.

\section{Polarity and Terminal Development}

Based on qualitative observation, we theorized that calyx formation is a polarized process, with terminals growing on the somatic surface opposite the eccentric nucleus. Our results demonstrate that this is the case and, moreover, terminal polarity becomes more extreme as terminal size increases. This suggests either the presence of trophic factor(s) on the principal cell surface opposite the nucleus position (CP), or inhibitory factors near the nucleus (NP), or both. Addition of principal cell surface area, particularly in the form of small protrusions called somatic spicules, is biased towards the $\mathrm{CP}$, though whether this growth is initiated by the principal cell or is a response to stimulation from the presynaptic terminal remains to be determined. If the growing protocalyx does induce postsynaptic changes that result in its polarized location, this would necessarily require a bias towards an initial contact for the axon 
with the CP and concomitant blockage of terminal spreading into the NP that results in anisotropic terminal growth. Our finding of rearrangement of glial coverage from P3-P4 to cover significantly more of the NP than earlier in development may provide an impediment to nuclear growth of terminals. This redistribution loses significance by P6 but may persist long enough to bias terminal growth. Dendrite location is also asymmetric, with our data showing $\sim 70 \%$ of dendrites located on the CP from P2-P9. A strong relationship between the axons of growing terminals and the dendrites of the principal cells was also evident, with $65-85 \%$ of axons for the two largest terminals per cell either tracking along a dendrite or first touching the somatic surface at the base of the dendrite. Intriguingly, significant differences in axodendritic association were found between cells with competing inputs and those with a single dominant input; while competing inputs tend to maintain an axodendritic association, only the largest input on cells with a single dominant terminal was significantly associated with a dendrite. Two potential theories can be drawn from this finding. First, it is possible that axodendritic association is a criterion for competing terminals, with axons not associated with a dendrite being removed early in development. Second, if principal cell dendrites function to capture globular bushy cell axons and guide them to the soma, then these dendrites may only be maintained until the cell has accrued axons capable of forming potential calyceal candidates and then removed. Surprisingly, we found two significant decreases in dendrite number which coincided temporally with the phases of competition in the MNTB, suggesting that as innervation becomes more refined, so too do the dendritic arbors of the principal cells. As adult principal cells possess dendrites, it is unclear if this dendritic pruning continues and then dendrites regrow or if the pruning ceases at a minimum number of dendrites. 


\section{Competition and Synaptic Silencing}

Using in vivo whole cell and juxtacellular recordings in P3-P4 rat pups, Sierksma et al. found that only 3 of 132 cells showed evidence of multiple terminals (2/67 for whole cell recordings for P3-P4, 1/65 for juxtacellular recordings at P4, Sierksma et al., 2017). There are several potential reasons why these numbers would be significantly lower than our ultrastructural findings. It is possible that the apparent difference in the number of competing terminals may represent a species difference in methods of circuit formation or in the time course of competition. As Sierksma et al. points out, whole cell recordings cannot differentiate between unique inputs with a high degree of certainty, meaning findings based on this technique are most likely an underestimate. Large terminals with very similar electrophysiological characteristics could also lead to an underestimate of polyinnervation. Perhaps most intriguingly, it is possible that multiple large terminals are present on the principal cell, but that all but one has been functionally silenced during competition. In the LSO-the major downstream target for the MNTB-supernumerary terminals are silenced a week prior to being removed (Kandler et al., 2009). Recently, Higashi et al. have demonstrated that BMP4 concentrated in dense core vesicles surrounding synapses is capable of destabilizing synapses in hippocampal neurons; as Xiao et al. showed defects in terminal pruning and electrophysiological profiles indicative of polyinnervation as late as P10 in BMP receptor knockout mice, this seems a likely pathway for terminal silencing (Xiao et al., 2013; Higashi et al., 2018). Interestingly, Higashi et al. found that reduced activity caused an increase in BMP4 expression in culture, suggesting that weaker terminals may be more susceptible to synapse destabilization. Decreasing membrane resistance and resting membrane potential in principal cells over development consistently raises the necessary strength of the presynaptic terminal required to elicit an action potential (Hoffpauir et al., 2010). This increasing postsynaptic threshold may induce synapse removal in terminals not capable of reaching it, either through 
BMP4-mediated destabilization or other methods of trans-synaptic signaling. The experiments performed by Higashi et al. are translatable to examination of the developing CH:MNTB system, and should be undertaken to explore this possibility (Higashi et al., 2018).

\section{Principal Cell Nucleus Eccentricity}

The results of this study demonstrate that nuclear eccentricity is a ubiquitous feature of MNTB principal cells throughout development. The functional consequences of this positioning on calyx development and competition have yet to be deciphered, however. As mentioned previously, the positioning of the nucleus may provide a method for transcriptional readout of presynaptic signal frequency through nuclear infolding and perinuclear positioning of voltagegated calcium channels (Bollmann et al., 1998; Queisser et al., 2011). Eccentric nuclear positioning may also create a directionality in protein production that focuses development on the cytoplasmic pole. The most expedient method for determining the necessity of nuclear eccentricity on $\mathrm{CH}: \mathrm{MNTB}$ development would be to disrupt the location of the principal cell nucleus. Nuclear position is mediated by attachment of the nucleoskeleton to the actin cytoskeleton of the cell through the LINC (Linker of Nucleoskeleton and Cytoskeleton) protein complex. This complex is composed of the inner nuclear membrane proteins Sun $1 / 2$ and the outer nuclear membrane transmembrane proteins Nesprin1-3, and these proteins link together within the perinuclear space via interaction of their KASH (Klarsicht, ANC-1, Syne Homology) domains. Razafsky et al. demonstrated the ability to disrupt nuclear position in cone cells using a KASH overexpression construct; unfortunately, crossing the $\mathrm{KASH}$-overexpressing mouse with the Engrailed-1-Cre mouse to disrupt nuclear eccentricity in MNTB principal cells was not successful despite the early expression of En1 (Fig. 50, Razafsky and Hodzic, 2009; Razafsky et al., 2012; Marrs et al., 2013). A similar lack of disruption was observed using this overexpression construct in Purkinje cells (Razafsky and Hodzic, 2014). Nuclear position may not change even after becoming untethered if the cytoskeleton is highly stable. However, given 
that En1 expression is evident as early as E13.5, before principal cells have migrated into their eventual position, it is highly unlikely that the cytoskeleton would remain stable enough to hold the nucleus in an eccentric position from this early stage. It is more likely that a secondary, asof-yet unidentified mechanism of nuclear anchoring is active in this system. Alternative methods of nuclear disruption, such as manipulations of the nuclear lamins, should be explored to decipher the role of nuclear position and morphology in $\mathrm{CH}$ :MNTB development.

\section{Broader Impact}

The $\mathrm{CH}$ :MNTB connection plays a vital role in early computation of binaural sound input. However, the usefulness in studying the development of this connection for understanding pathological conditions such as congenital deafness is not immediately apparent; as mentioned previously, congenitally deaf mice $(d n / d n)$ show no defects in calyx formation as late as P20, and electrophysiological differences in these animals have only been noted after hearing onset would have occurred (Leão et al., 2006a, 2006b; Youssoufian et al., 2008). This does not discount the potential for the development of this system to have an impact on our understanding of auditory neuroscience, but more intensive characterization of the structure and function of the $\mathrm{CH}$ :MNTB connection at early postnatal ages in genetic models of deafness are needed. This research may provide significant insight into the development-and potential treatment—of connectivity disorders, however. Compelling evidence points to deficits in functional refinement of the central nervous system in disorders such as autism and schizophrenia (Lynall et al., 2010; Supekar et al., 2013). Moreover, these connectivity disorders share a high degree of comorbidity with auditory dysfunction, particularly at the level of the auditory brainstem (Tomchek and Dunn, 2007; Källstrand et al., 2012). MNTB neurons in autistic brains from children showed a decrease in size and number, and possess a more immature morphology (Kulesza et al., 2011). Several proteins involved in calyx development have also been associated with autism spectrum disorders. For example, miR-96 has recently 
been identified as an autism biomarker through a bioinformatics approach comparing differential mRNA expression in autistics with known regulatory functions of miRNAs (Shen et al., 2016). Neuroligin-3, a member of a family of proteins frequently mutated in autism, significantly altered EPSC amplitude and kinetics when knocked out in principal cells using a PV-Cre mouse line (Zhang et al., 2016). Autism spectrum disorder is also highly comorbid with Fragile X syndrome, the most common cause of mental retardation caused by mutations in the eponymous Fragile $\mathrm{X}$ Mental Retardation protein (FMR or FMRP) (O'Donnell and Warren, 2002; Belmonte and Bourgeron, 2006). In FMR knockout mice, MNTB principal cell size is reduced similar to that of autistic individuals, GABAergic inhibition is increased, and the tonotopic expression of $\mathrm{K}_{v} 3.1 \mathrm{~b}$ channels is abolished (Strumbos et al., 2010; Rotschafer et al., 2015). Adult calyces in FMR knockouts are also significantly greater in volume and surface area than control animals (Wang et al., 2015). In relation to this work, FMR trafficking has been shown to require both the LGL (lethal giant larva) and the Par complex, suggesting FMR may be transported in a polarityspecific manner (Zarnescu et al., 2005). Additionally, our findings suggest coordinated calyx and principal cell maturation marked by significant growth of the principal cell; this phase of growth may be absent in cases of autism spectrum disorder and fragile $\mathrm{X}$ mental retardation given the consistently small principal cell size found in these studies. Further study will be necessary to determine if either competition or polarity in $\mathrm{CH}$ :MNTB development is disrupted in animal models of these disorders. If this is the case, however, understanding the key mechanisms in this developmental process may help clarify the etiology of these disorders and-possibly—lead to new avenues of treatment. 


\section{References}

Abdul-latif ML, Salazar JAA, Marshak S, Dinh ML, Cramer KS (2015) Ephrin-A2 and ephrin-A5 guide contralateral targeting but not topographic mapping of ventral cochlear nucleus axons. Neural Dev 10:27.

Ahern CA, Payandeh J, Bosmans F, Chanda B (2016) The hitchhiker's guide to the voltagegated sodium channel galaxy. J Gen Physiol 147:1-24.

Ariel Gomez L, Brusco A, Saavedra JP (1990) Immunocytochemical study of S-100 positive glial cells in the brainstem and spinal cord of the rat embryo. Int J Dev Neurosci 8:55-64.

Armengol JA, Sotelo C (1991) Early dendritic development of Purkinje cells in the rat cerebellum. A light and electron microscopic study using axonal tracing in "in vitro" slices. Dev Brain Res 64:95-114.

Arnott RH, Wallace MN, Shackleton TM, Palmer AR (2004) Onset Neurones in the Anteroventral Cochlear Nucleus Project to the Dorsal Cochlear Nucleus. JARO - J Assoc Res Otolaryngol 5:153-170.

Awatramani GB, Price GD, Trussell LO (2005a) Modulation of transmitter release by presynaptic resting potential and background calcium levels. Neuron 48:109-121.

Awatramani GB, Turecek R, Trussell LO (2005b) Staggered development of GABAergic and glycinergic transmission in the MNTB. J Neurophysiol 93:819-828.

Balice-Gordon R, Lichtman J (1990) In vivo visualization of the growth of pre-and postsynaptic elements of neuromuscular junctions in the mouse. J Neurosci 10:894-908.

Balice-Gordon R, Lichtman J (1993) In vivo observations of pre-and postsynaptic changes during the transition from multiple to single innervation at developing neuromuscular junctions. J Neurosci 73:834-855.

Banks MI, Smith PH (1992) Intracellular recordings from neurobiotin-labeled cells in brain slices of the rat medial nucleus of the trapezoid body. J Neurosci 12:2819-2837.

Barnes AP, Polleux F (2009) Establishment of axon-dendrite polarity in developing neurons. Annu Rev Neurosci 32:347-381.

Baydyuk M, Xu J, Wu LG (2016) The calyx of Held in the auditory system: Structure, function, and development. Hear Res 338:22-31.

Belmonte MK, Bourgeron T (2006) Fragile X syndrome and autism at the intersection of genetic and neural networks. Nat Neurosci 9:1221-1225.

Bergsman JB, De Camilli P, McCormick D a (2004) Multiple large inputs to principal cells in the mouse medial nucleus of the trapezoid body. J Neurophysiol 92:545-552.

Bishop DL, Misgeld T, Walsh MK, Gan W-B, Lichtman JW (2004) Axon branch removal at developing synapses by axosome shedding. Neuron 44:651-661.

Bock DD, Lee W-CA, Kerlin AM, Andermann ML, Hood G, Wetzel AW, Yurgenson S, Soucy ER, Kim HS, Reid RC (2011) Network anatomy and in vivo physiology of visual cortical neurons. Nature 471:177-182.

Bollmann JH, Helmchen F, Borst JG, Sakmann B (1998) Postsynaptic Ca2+ influx mediated by 
three different pathways during synaptic transmission at a calyx-type synapse. J Neurosci 18:10409-10419.

Bornens M, Azimzadeh J (2007) Origin and evolution of the centrosome. Adv Exp Med Biol 607:119-129.

Borst JG, Helmchen F, Sakmann B (1995) Pre- and postsynaptic whole-cell recordings in the medial nucleus of the trapezoid body of the rat. J Physiol 489:825-840.

Borst JGG, Sakmann B (1996) Calcium influx and transmitter release in a fast CNS synapse. Nature 383:431-434.

Borst JGG, Soria van Hoeve J (2012) The calyx of held synapse: from model synapse to auditory relay. Annu Rev Physiol 74:199-224.

Boudreau JC, Tsuchitani C (1968) Binaural interaction in the cat superior olive S segment. J Neurophysiol 31:442-454.

Boulter J, Hollmann M, O'Shea-Greenfield A, Hartley M, Deneris E, Maron C, Heinemann S (1990) Molecular Cloning and Functional Expression of Glutamate Receptor Subunit Genes. Science (80) 249:1033-1037.

Bouyain S, Watkins DJ (2010) The protein tyrosine phosphatases PTPRZ and PTPRG bind to distinct members of the contactin family of neural recognition molecules. Proc Natl Acad Sci 107:2443-2448.

Brand A, Behrend O, Marquardt T, McAlpine D, Grothe B (2002) Precise inhibition is essential for microsecond interaural time difference coding. Nature 417:543-547.

Brandebura AN, Morehead M, Heller DT, Holcomb PS, Kolson DR, Jones G, Mathers PH, Spirou GA (2018) Glial Cell Expansion Coincides with Neural Circuit Formation in the Developing Auditory Brainstem. Dev Neurobiol:1-20.

Brew HM, Forsythe ID (1995) Two voltage-dependent K+ conductances with complementary functions in postsynaptic integration at a central auditory synapse. J Neurosci 15:80118022.

Briggman KL, Bock DD (2012) Volume electron microscopy for neuronal circuit reconstruction. Curr Opin Neurobiol 22:154-161.

Briggman KL, Helmstaedter M, Denk W (2011) Wiring specificity in the direction-selectivity circuit of the retina. Nature 471:183-188.

Brown M, Jansen J, Essen D Van (1976) Polyneuronal innervation of skeletal muscle in newborn rats and its elimination during maturation. J Physiol 261:387-422.

Brownell WE (1975) Organization of the cat trapezoid body and the discharge characteristics of its fibers. Brain Res 94:413-433.

Brungart DS, Rabinowitz WM (1999) Auditory localization of nearby sources. Head-related transfer functions. J Acoust Soc Am 106:1465-1479.

Buffelli M, Burgess R, Feng G (2003) Genetic evidence that relative synaptic efficacy biases the outcome of synaptic competition. Nature 424:430-434.

Buffo A, Rossi F (2013) Origin, lineage and function of cerebellar glia. Prog Neurobiol 109:4263. 
Caicedo A, Eybalin M (1999) Glutamate receptor phenotypes in the auditory brainstem and midbrain of the developing rat. Eur J Neurosci 11:51-74.

Caird D, Klinke R (1983) Processing of binaural stimuli by cat superior olivary complex neurons. Exp Brain Res 52:385-399.

Calderon de Anda F, Gärtner A, Tsai L-H, Dotti CG (2008) Pyramidal neuron polarity axis is defined at the bipolar stage. J Cell Sci 121:178-185.

Cant N, Morest D (1979) The bushy cells in the anteroventral cochlear nucleus of the cat. A study with the electron microscope. Nat Neurosci 4:1925-1945.

Cant NB, Benson CG (2003) Parallel auditory pathways: Projection patterns of the different neuronal populations in the dorsal and ventral cochlear nuclei. Brain Res Bull 60:457-474.

Chedotal A, Sotelo C (1992) Early Development of Olivocerebellar Projections in the Fetal Rat Using CGRP Immunocytochemistry. Eur J Neurosci 4:1159-1179.

Chen C, Regehr W (2000) Developmental remodeling of the retinogeniculate synapse. Neuron 28:955-966.

Chen Z, Gore BB, Long H, Ma L, Tessier-Lavigne M (2008) Alternative Splicing of the Robo3 Axon Guidance Receptor Governs the Midline Switch from Attraction to Repulsion. Neuron 58:325-332.

Cignoni P, Callieri M, Corsini M, Dellepiane M, Ganovelli F, Ranzuglia G (2008) MeshLab: an Open-Source Mesh Processing Tool. In: Sixth Eurographics Italian Chapter Conference, pp 129-136.

Claesdotter- Hybbinette E, Cervin M, Åkerlund S, Råstam M, Lindvall M (2016) Gender Specific Differences in Auditory Brain Stem Response in Young Patients with ADHD. J Neuropsychiatr 2:28-35.

Clancy B, Darlington RB, Finlay BL (2001) Translating developmental time across mammalian species. Neuroscience 105:7-17.

Clause A, Kim G, Sonntag M, Weisz CJCJC, Vetter DEE, Rübsamen R, Kandler K, Rubsamen $\mathrm{R}$, Kandler K (2014) The Precise Temporal Pattern of Prehearing Spontaneous Activity Is Necessary for Tonotopic Map Refinement. Neuron 82:822-835.

Colman H, Nabekura J, Lichtman J (1997) Alterations in synaptic strength preceding axon withdrawal. Science (80- ) 275:356-361.

Coppola T, Magnin-Lüthi S, Perret-Menoud V, Gattesco S, Schiavo G, Regazzi R (2001) Direct Interaction of the Rab3 Effector RIM with Ca2+ Channels, SNAP-25, and Synaptotagmin. J Biol Chem 276:32756-32762.

Cramer KS, Rubel EW (2016) Glial Cell Contributions to Auditory Brainstem Development. Front Neural Circuits 10:1-8.

Cull-Candy S, Brickley S, Farrant M (2001) NMDA receptor subunits: diversity, development and disease. Curr Opin Neurobiol 11:327-335.

Dai J, Chen P, Tian H, Sun J (2015) Spontaneous Vesicle Release Is Not Tightly Coupled to Voltage-Gated Calcium Channel-Mediated Ca2+ Influx and Is Triggered by a Ca2+ Sensor Other Than Synaptotagmin-2 at the Juvenile Mice Calyx of Held Synapses. J Neurosci 35:9632-9637. 
Das A, Gajendra S, Falenta K, Oudin MJ, Peschard P, Feng S, Wu B, Marshall CJ, Doherty P, Guo W, Lalli G (2014) RalA promotes a direct exocyst-Par6 interaction to regulate polarity in neuronal development. J Cell Sci 127:686-699.

Davis KA (2005) Contralateral effects and binaural interactions in dorsal cochlear nucleus. JARO - J Assoc Res Otolaryngol 6:280-296.

Dayaratne MWN, Vlajkovic SM, Lipski J, Thorne PR (2014) Kölliker's Organ and the Development of Spontaneous Activity in the Auditory System : Implications for Hearing Dysfunction. Biomed Res Int 2014:1-8.

Deerinck T, Bushong E, Thor A, Ellisman M (2010) NCMIR methods for 3D EM: a new protocol for preparation of biological specimens for serial block face scanning electron microscopy. Microscopy:6-8.

Denk W, Horstmann H (2004) Serial block-face scanning electron microscopy to reconstruct three-dimensional tissue nanostructure. PLoS Biol 2:e329.

Desesso JM, Scialli AR, Holson JF (1999) Apparent Lability of Neural Tube Closure in Laboratory Animals and Humans. 162:143-162.

Di Bonito M, Narita Y, Avallone B, Sequino L, Mancuso M, Andolfi G, Franzè AM, Puelles L, Rijli FM, Studer M (2013) Assembly of the auditory circuitry by a Hox genetic network in the mouse brainstem. PLoS Genet 9:e1003249.

Dinh ML, Koppel SJ, Korn MJ, Cramer KS (2014) Distribution of glial cells in the auditory brainstem: Normal development and effects of unilateral lesion. Neuroscience 278:237252.

Dodson PD, Barker MC, Forsythe ID (2002) Two heteromeric Kv1 potassium channels differentially regulate action potential firing. J Neurosci 22:6953-6961.

Dong W, Radulovic T, Goral RO, Thomas C, Suarez Montesinos M, Guerrero-Given D, Hagiwara A, Putzke T, Hida Y, Abe M, Sakimura K, Kamasawa N, Ohtsuka T, Young SM (2018) CAST/ELKS Proteins Control Voltage-Gated Ca2+Channel Density and Synaptic Release Probability at a Mammalian Central Synapse. Cell Rep 24:284-293.e6.

Doucet JR, Ryugo DK (2006) Structural and functional classes of multipolar cells in the ventral cochlear nucleus. Anat Rec - Part A Discov Mol Cell Evol Biol 288:331-344.

Duman JG, Tzeng CP, Tu Y-K, Munjal T, Schwechter B, Ho TS-Y, Tolias KF (2013) The adhesion-GPCR BAI1 regulates synaptogenesis by controlling the recruitment of the Par3/Tiam1 polarity complex to synaptic sites. J Neurosci 33:6964-6978.

Dunah AW, Hueske E, Wyszynski M, Hoogenraad CC, Jaworski J, Pak DT, Simonetta A, Liu G, Sheng M (2005) LAR receptor protein tyrosine phosphatases in the development and maintenance of excitatory synapses. Nat Neurosci 8:458-467.

Dunn ME, Schilling K, Mugnaini E (1998) Development and fine structure of murine Purkinje cells in dissociated cerebellar cultures : neuronal polarity. Anat Embryol (Berl) 197:9-29.

Ehret G (1976) Development of absolute auditory thresholds in the house mouse (Mus musculus). J Am Audiol Soc 1:179-184.

Ehret G (2005) Infant rodent ultrasounds - A gate to the understanding of sound communication. Behav Genet 35:19-29. 
Elezgarai I, Bilbao A, Mateos JM, Azkue JJ, Benítez R, Osorio A, Díez J, Puente N, DoñateOliver F, Grandes P (2001) Group II metabotropic glutamate receptors are differentially expressed in the medial nucleus of the trapezoid body in the developing and adult rat. Neuroscience 104:487-498.

Elezgarai I, Díez J, Puente N, Azkue JJ, Benítez R, Bilbao A, Knöpfel T, Doñate-Oliver F, Grandes P (2003) Subcellular localization of the voltage-dependent potassium channel $\mathrm{Kv3} .1 \mathrm{~b}$ in postnatal and adult rat medial nucleus of the trapezoid body. Neuroscience 118:889-898.

Erazo-Fischer E, Striessnig J, Taschenberger H (2007) The Role of Physiological Afferent Nerve Activity during In Vivo Maturation of the Calyx of Held Synapse. J Neurosci 27:1725-1737.

Etienne-Manneville S, Hall A (2003) Cell polarity: Par6, aPKC and cytoskeletal crosstalk. Curr Opin Cell Biol 15:67-72.

Evans TA, Bashaw GJ (2010) Axon guidance at the midline: of mice and flies. Curr Opin Neurobiol 20:79-85.

Fan F, Funk L, Lou X (2016) Dynamin 1- and 3-Mediated Endocytosis Is Essential for the Development of a Large Central Synapse In Vivo. J Neurosci 36:6097-6115.

Farago AF, Awatramani RB, Dymecki SM (2006) Assembly of the brainstem cochlear nuclear complex is revealed by intersectional and subtractive genetic fate maps. Neuron 50:205218.

Favero M, Busetto G, Cangiano A (2012) Spike timing plays a key role in synapse elimination at the neuromuscular junction. Proc Natl Acad Sci U S A 109:E1667-75.

Fedchyshyn MJ, Wang L-Y (2005) Developmental Transformation of the Release Modality at the Calyx of Held Synapse. J Neurosci 25:4131-4140.

Ford MC, Grothe B, Klug A (2009) Fenestration of the calyx of Held occurs sequentially along the tonotopic axis, is influenced by afferent activity, and facilitates glutamate clearance. $J$ Comp Neurol 514:92-106.

Forsythe ID (1994) Direct patch recording from identified presynaptic terminals mediating glutamatergic EPSCs in the rat CNS, in vitro. J Physiol 479:381-387.

Forsythe ID, Barnes-Davies M (1993) The binaural auditory pathway: excitatory amino acid receptors mediate dual timecourse excitatory postsynaptic currents in the rat medial nucleus of the trapezoid body. Proc Biol Sci 251:151-157.

Friauf E, Ostwald J (1988) Divergent projections of physiologically characterized rat ventral cochlear nucleus neurons as shown by intra-axonal injection of horseradish peroxidase. Exp brain Res 73:263-284.

Friocourt F, Chédotal A (2017) The Robo3 receptor, a key player in the development, evolution, and function of commissural systems. Dev Neurobiol 77:876-890.

Furukawa H, Singh SK, Mancusso R, Gouaux E (2005) Subunit arrangement and function in NMDA receptors. Nature 438:185-192.

Futai K, Okada M, Matsuyama K, Takahashi T (2001) High-fidelity transmission acquired via a developmental decrease in NMDA receptor expression at an auditory synapse. J Neurosci 
21:3342-3349.

Galambos R, Schwartzkopff J, Rupert A (1959) Microelectrode study of superior olivary nuclei. Am J Physiol 197:527-536.

Gallo V, Zhou JM, McBain CJ, Wright P, Knutson PL, Armstrong RC (1996) Oligodendrocyte progenitor cell proliferation and lineage progression are regulated by glutamate receptormediated K+ channel block. J Neurosci 16:2659-2670.

Goldberg J, Brown P (1969) Response of Binaural neurons of Dog Superior Olivary Complex to Dichotic Tonal Stimuli: Some Physiological Mechanisms of Sound Localization. J Physiol 32:613-636.

Graham A, Papalopulu N, Krumlauf R, Ridgeway T, Hill M (1989) The Murine and Drosophila Homeobox Complexes Have Common Features of Organization and Expression. Cell 57:367-378.

Grizel A V., Glukhov GS, Sokolova OS (2014) Mechanisms of activation of voltage-gated potassium channels. Acta Naturae 6:10-26.

Grothe B, Pecka M, McAlpine D (2010) Mechanisms of Sound Localization in Mammals. Physiol Rev 90:983-1012.

Hachem S, Aguirre A, Vives V, Marks A, Gallo V, Legraverend C (2005) Spatial and temporal expression of S100B in cells of oligodendrocyte lineage. Glia 51:81-97.

Hackney CM, Osen KK, Kolston J (1990) Anatomy of the cochlear nuclear complex of guinea pig. Anat Embryol (Berl) 182:123-149.

Hammerschmidt K, Reisinger E, Westekemper K, Ehrenreich L, Strenzke N, Fischer J (2012) Mice do not require auditory input for the normal development of their ultrasonic vocalizations. BMC Neurosci 13.

Hamori J, Jakab RL, Takacs J (1997) Morphogenetic plasticity of neuronal elements in cerebellar glomeruli during deafferentation-induced synaptic reorganization. J Neural Transplant Plast 6:11-20.

Han Y, Babai N, Kaeser P, Südhof TC, Schneggenburger R (2015) RIM1 and RIM2 redundantly determine $\mathrm{Ca}^{2+}$ channel density and readily releasable pool size at a large hindbrain synapse. J Neurophysiol 113:255-263.

Han Y, Kaeser PS, Südhof TC, Schneggenburger R (2011) RIM determines Ca2+channel density and vesicle docking at the presynaptic active zone. Neuron 69:304-316.

Hashimoto K, Ichikawa R, Kitamura K, Watanabe M, Kano M (2009) Translocation of a "winner" climbing fiber to the Purkinje cell dendrite and subsequent elimination of "losers" from the soma in developing cerebellum. Neuron 63:106-118.

Hee Kim J, Kushmerick C, von Gersdorff H (2010) Presynaptic Resurgent Na+ Currents Sculpt the Action Potential Waveform and Increase Firing Reliability at a CNS Nerve Terminal. J Neurosci 30:15479-15490.

Hegarty S V., Sullivan AM, O’Keeffe GW (2017) Endocytosis contributes to BMP2-induced Smad signalling and neuronal growth. Neurosci Lett 643:32-37.

Held H (1893) Die centrale Gehörleitung. Arch Anat Physiol (Anat Abt). 
Henning GB (1974) Detectability of interaural delay in high-frequency complex waveforms. J Acoust Soc Am 55:84-90.

Hermida D, Elezgarai I, Puente N, Alonso V, Anabitarte N, Bilbao A, Doñate-Oliver F, Grandes $P$ (2006) Developmental increase in postsynaptic alpha-amino-3-hydroxy-5-methyl-4 isoxazolepropionic acid receptor compartmentalization at the calyx of Held synapse. $\mathrm{J}$ Comp Neurol 495:624-634.

Hermida D, Mateos JM, Elezgarai I, Puente N, Bilbao A, Bueno-López JL, Streit P, Grandes P (2010) Spatial compartmentalization of AMPA glutamate receptor subunits at the calyx of Held synapse. J Comp Neurol 518:163-174.

Higashi T, Tanaka S, lida T, Okabe S (2018) Synapse Elimination Triggered by BMP4 Exocytosis and Presynaptic BMP Receptor Activation. Cell Rep 22:919-929.

Higginbotham HR, Gleeson JG (2007) The centrosome in neuronal development. Trends Neurosci 30:276-283.

Hirano Y, Yoshinaga S, Takeya R, Suzuki NN, Horiuchi M, Kohjima M, Sumimoto H, Inagaki F (2005) Structure of a cell polarity regulator, a complex between atypical PKC and Par6 PB1 domains. J Biol Chem 280:9653-9661.

Hirrlinger J, Hülsmann S, Kirchhoff F (2004) Astroglial processes show spontaneous motility at active synaptic terminals in situ. Eur J Neurosci 20:2235-2239.

Hirtz JJ, Boesen M, Braun N, Deitmer JW, Kramer F, Lohr C, Müller B, Nothwang HG, Striessnig J, Löhrke S, Friauf E (2011) Cav1.3 calcium channels are required for normal development of the auditory brainstem. J Neurosci 31:8280-8294.

Hoffpauir BK, Grimes JL, Mathers PH, Spirou GA (2006) Synaptogenesis of the calyx of Held: rapid onset of function and one-to-one morphological innervation. J Neurosci 26:55115523.

Hoffpauir BK, Kolson DR, Mathers PH, Spirou GA (2010) Maturation of synaptic partners: functional phenotype and synaptic organization tuned in synchrony. J Physiol 588:43654385.

Holcomb PS, Deerinck TJ, Ellisman MH, Spirou GA (2013a) Construction of a polarized neuron. J Physiol 591:3145-3150.

Holcomb PS, Hoffpauir BK, Hoyson MC, Jackson DR, Deerinck TJ, Marrs GS, Dehoff M, Wu J, Ellisman MH, Spirou GA (2013b) Synaptic inputs compete during rapid formation of the calyx of Held: a new model system for neural development. J Neurosci 33:12954-12969.

Hollander M, Wolfe D (1999) Nonparametric Statistical Methods, 2nd ed. New York: John Wiley \& Sons.

Hong YK, Chen C (2011) Wiring and rewiring of the retinogeniculate synapse. Curr Opin Neurobiol 21:228-237.

Hooks BM, Chen C (2006) Distinct roles for spontaneous and visual activity in remodeling of the retinogeniculate synapse. Neuron 52:281-291.

Horstmann H, Körber C, Sätzler K, Aydin D, Kuner T (2012) Serial Section Scanning Electron Microscopy (S3EM) on Silicon Wafers for Ultra-Structural Volume Imaging of Cells and Tissues Fox MA, ed. PLoS One 7:e35172. 
Howell D, Morgan W, Jarjour A, Spirou G, Berrebi A, Kennedy T, Mathers P (2007) Molecular guidance cues necessary for axon pathfinding from the ventral cochlear nucleus. J Comp Neurol 549:533-549.

Hsieh CY, Nakamura PA, Luk SO, Miko IJ, Henkemeyer M, Cramer KS (2010) Ephrin-B reverse signaling is required for formation of strictly contralateral auditory brainstem pathways. $\mathrm{J}$ Neurosci 30:9840-9849.

Hunt P, Whiting J, Muchamore I, Marshall H, Krumlauf R (1991) Homeobox genes and models for patterning the hindbrain and branchial arches. Development 113:187-196.

Hunter NL, Dymecki SM (2007) Molecularly and temporally separable lineages form the hindbrain roof plate and contribute differentially to the choroid plexus. Development 134:3449-3460.

lino M, Goto K, Kakegawa W, Okado H, Sudo M, Ishiuchi S, Miwa a, Takayasu Y, Saito I, Tsuzuki K, Ozawa S (2001) Glia-synapse interaction through Ca2+-permeable AMPA receptors in Bergmann glia. Science 292:926-929.

Irving C, Flenniken A, Alldus G, Wilkinson D (1996) Cell-cell interactions and segmentation in the developing vertebrate hindbrain. Biochem Soc Symp 62:85-95.

Ishikawa T, Nakamura Y, Saitoh N, Li W-B, Iwasaki S, Takahashi T (2003) Distinct roles of Kv1 and Kv3 potassium channels at the calyx of Held presynaptic terminal. J Neurosci 23:10445-10453.

Ishikawa Y, Yamamoto N, Yoshimoto M, Ito H (2012) The primary brain vesicles revisited: Are the three primary vesicles (forebrain/midbrain/hindbrain) universal in vertebrates? Brain Behav Evol 79:75-83.

Iwasaki S, Momiyama A, Uchitel O, Takahashi T (2000) Developmental changes in calcium channel types mediating central synaptic transmission. J Neurosci 20:59-65.

Iwasaki S, Takahashi T (1998) Developmental changes in calcium channel types mediating synaptic transmission in rat auditory brainstem. J Physiol 509 (Pt 2):419-423.

Jacobson AG, Tam PPL (1982) Cephalic neurulation in the mouse embryo analyzed by SEM and morphometry. Anat Rec 203:375-396.

Jaworski A, Long H, Tessier-Lavigne M (2010) Collaborative and Specialized Functions of Robo1 and Robo2 in Spinal Commissural Axon Guidance. J Neurosci 30:9445-9453.

Jean-Baptiste M, Morest DK (1975) Transneuronal changes of synaptic endings and nuclear chromatin in the trapezoid body following cochlear ablations in cats. J Comp Neurol 162:111-133.

Jeffress $L$ (1948) A place theory of sound localization. J Comp Physiol Psychol 41:35-39.

Jimenez-Guri E, Udina F, Colas JF, Sharpe J, Padrón-Barthe L, Torres M, Pujades C (2010) Clonal analysis in mice underlines the importance of rhombomeric boundaries in cell movement restriction during hindbrain segmentation. PLoS One 5:1-7.

Jing L, Lefebvre JL, Gordon LR, Granato M (2009) Wnt Signals Organize Synaptic Prepattern and Axon Guidance through the Zebrafish unplugged/MuSK Receptor. Neuron 61:721733.

Johnson JW, Ascher P (1987) Glycine potentiates the NMDA response in cultured mouse brain 
neurons. Nature 325:529-531.

Joris PX, Carney LH, Smith PH, Yin TC (1994) Enhancement of neural synchronization in the anteroventral cochlear nucleus. I. Responses to tones at the characteristic frequency. J Neurophysiol 71:1022-1036.

Joshi I, Shkralla S, Titis P, Wang L-Y (2004) The Role of AMPA Receptor Gating in the Development of High-Fidelity Neurotransmission at the Calyx of Held Synapse. J Neurosci 24:183-196.

Joshi I, Wang L-Y (2002) Developmental profiles of glutamate receptors and synaptic transmission at a single synapse in the mouse auditory brainstem. J Physiol 540:861-873.

Joshi I, Yang Y-M, Wang L-Y (2007) Coincident Activation of Metabotropic Glutamate Receptors and NMDA Receptors (NMDARs) Downregulates Perisynaptic/Extrasynaptic NMDARs and Enhances High-Fidelity Neurotransmission at the Developing Calyx of Held Synapse. J Neurosci 27:9989-9999.

Kaeser PS, Regehr WG (2017) The readily releasable pool of synaptic vesicles. Curr Opin Neurobiol 43:63-70.

Källstrand J, Nehlstedt SF, Sköld ML, Nielzén S (2012) Lateral asymmetry and reduced forward masking effect in early brainstem auditory evoked responses in schizophrenia. Psychiatry Res 196:188-193.

Kamiya K, Takahashi K, Kitamura K, Momoi T, Yoshikawa Y (2001) Mitosis and apoptosis in postnatal auditory system of the C3H/He strain. Brain Res 901:296-302.

Kampa BM, Clements J, Jonas P, Stuart GJ (2004) Kinetics of Mg2+ unblock of NMDA receptors: implications for spike-timing dependent synaptic plasticity. J Physiol 556:337345.

Kanamori T, Yoshino J, Yasunaga KI, Dairyo Y, Emoto K (2015) Local endocytosis triggers dendritic thinning and pruning in Drosophila sensory neurons. Nat Commun 6:1-14.

Kandler K, Clause A, Noh J (2009) Tonotopic reorganization of developing auditory brainstem circuits. Nat Neurosci 12:711-717.

Kandler K, Friauf E (1993) Pre- and postnatal development of efferent connections of the cochlear nucleus in the rat. J Comp Neurol 328:161-184.

Kano M, Hashimoto K (2012) Activity-dependent maturation of climbing fiber to Purkinje cell synapses during postnatal cerebellar development. Cerebellum 11:449-450.

Kar UP, Dey H, Rahaman A (2017) Regulation of dynamin family proteins by post-translational modifications. J Biosci 42:333-344.

Káradóttir R, Attwell D (2007) Neurotransmitter receptors in the life and death of oligodendrocytes. Neuroscience 145:1426-1438.

Kasthuri N, Lichtman JW (2003) The role of neuronal identity in synaptic competition. Nature 424:426-430.

Katagiri T, Watabe T (2016) Bone morphogenetic proteins. Cold Spring Harb Perspect Biol.

Kazhdan M, Bolitho M, Hoppe H (2006) Poisson surface reconstruction. Eurographics Symp Geom Process:61-70. 
Kazhdan M, Hoppe H (2013) Screened poisson surface reconstruction. ACM Trans Graph $32: 1-13$.

Kharfallah F, Guyot MC, El Hassan AR, Allache R, Merello E, De Marco P, Di Cristo G, Capra V, Kibar Z (2017) Scribble1 plays an important role in the pathogenesis of neural tube defects through its mediating effect of Par-3 and Vangl1/2 localization. Hum Mol Genet 26:2307-2320.

Kil J, Hkageyama G, Semple MN, Kitzes LM (1995) Development of ventral cochlear nucleus projections to the superior olivary complex in gerbil. J Comp Neurol 353:317-340.

Kimura M, Saitoh N, Takahashi T (2003) Adenosine A1 receptor-mediated presynaptic inhibition at the calyx of Held of immature rats. J Physiol 553:415-426.

Klein R (2004) Eph/ephrin signaling in morphogenesis, neural development and plasticity. Curr Opin Cell Biol 16:580-589.

Kleinfeld D, Bharioke A, Blinder P, Bock DD, Briggman KL, Chklovskii DB, Denk W, Helmstaedter M, Kaufhold JP, Lee W-CA, Meyer HS, Micheva KD, Oberlaender M, Prohaska S, Reid RC, Smith SJ, Takemura S, Tsai PS, Sakmann B (2011) Large-scale automated histology in the pursuit of connectomes. J Neurosci 31:16125-16138.

Kochubey O, Han Y, Schneggenburger R (2009) Developmental regulation of the intracellular $\mathrm{Ca}^{2+}$ sensitivity of vesicle fusion and $\mathrm{Ca}^{2+}$-secretion coupling at the rat calyx of Held. $\mathrm{J}$ Physiol 587:3009-3023.

Kodani A, Tonthat V (2010) Par6a Interacts with the Dynactin Subunit p150Glued and Is a Critical Regulator of Centrosomal Protein Recruitment. Mol Biol Cell 21:3376-3385.

Koike-Tani M, Kanda T, Saitoh N, Yamashita T, Takahashi T (2008) Involvement of AMPA receptor desensitization in short-term synaptic depression at the calyx of Held in developing rats. J Physiol 586:2263-2275.

Koike-Tani M, Saitoh N, Takahashi T (2005) Mechanisms Underlying Developmental Speeding in AMPA-EPSC Decay Time at the Calyx of Held. J Neurosci 25:199-207.

Kolson DR, Wan J, Wu J, Dehoff M, Brandebura AN, Qian J, Mathers PH, Spirou GA (2016) Temporal patterns of gene expression during calyx of held development. Dev Neurobiol 76:166-189.

Kronander E, Michalski N, Lebrand C, Hornung JP, Schneggenburger R (2017) An organotypic slice culture to study the formation of calyx of Held synapses in-vitro. PLoS One 12:1-19.

Kuhn S, Johnson SL, Furness DN, Chen J, Ingham N, Hilton JM, Steffes G, Lewis MA, Zampini V, Hackney CM, Masetto S, Holley MC, Steel KP, Marcotti W (2011) miR-96 regulates the progression of differentiation in mammalian cochlear inner and outer hair cells. Proc Natl Acad Sci 108:2355-2360.

Kulesza RJ, Lukose R, Stevens LV (2011) Malformation of the human superior olive in autistic spectrum disorders. Brain Res 1367:360-371.

Kuwabara N, DiCaprio RA, Zook JM (1991) Afferents to the medial nucleus of the trapezoid body and their collateral projections. J Comp Neurol 314:684-706.

Kuwabara N, Zook J (1992) Projections to the medial superior olive from the medial and lateral nuclei of the trapezoid body in rodents and bats. J Comp Neurol 324:522-538. 
Lalli G (2009) RalA and the exocyst complex influence neuronal polarity through PAR-3 and aPKC. J Cell Sci 122:1499-1506.

Leão R, Kushmerick C, Pinaud R, Renden R, Li G-L, Taschenberger H, Spirou G, Levinson S, von Gersdorff H (2005) Presynaptic Na+ Channels: Locus, Development, and Recovery from Inactivation at a High-Fidelity Synapse. J Neurosci 25:3724-3738.

Leão RN, Berntson A, Forsythe ID, Walmsley B (2004a) Reduced low-voltage activated K+ conductances and enhanced central excitability in a congenitally deaf $(\mathrm{dn} / \mathrm{dn})$ mouse. $\mathrm{J}$ Physiol 559:25-33.

Leão RN, Naves MM, Leão KE, Walmsley B (2006a) Altered sodium currents in auditory neurons of congenitally deaf mice. Eur J Neurosci 24:1137-1146.

Leão RN, Oleskevich S, Sun H, Bautista M, Fyffe REW, Walmsley B (2004b) Differences in glycinergic mIPSCs in the auditory brain stem of normal and congenitally deaf neonatal mice. J Neurophysiol 91:1006-1012.

Leão RN, Sun H, Svahn K, Berntson A, Youssoufian M, Paolini AG, Fyffe REW, Walmsley B (2006b) Topographic organization in the auditory brainstem of juvenile mice is disrupted in congenital deafness. J Physiol 571:563-578.

Lee CW, Eglen SJ, Wong ROL (2002) Segregation of ON and OFF retinogeniculate connectivity directed by patterned spontaneous activity. J Neurophysiol 88:2311-2321.

Lewis MA, Quint E, Glazier AM, Fuchs H, De Angelis MH, Langford C, Van Dongen S, AbreuGoodger C, Piipari M, Redshaw N, Dalmay T, Moreno-Pelayo MA, Enright AJ, Steel KP (2009) An ENU-induced mutation of miR-96 associated with progressive hearing loss in mice. Nat Genet 41:614-618.

Li L, Liu S, Lei Y, Cheng Y, Yao C, Zhen X (2014) Robo3.1A suppresses slit-mediated repulsion by triggering degradation of Robo2. J Neurosci Res 92:835-846.

Li W, Kaczmarek LK, Perney TM (2001) Localization of two high-threshold potassium channel subunits in the rat central auditory system. J Comp Neurol 437:196-218.

Lilley BN, Krishnaswamy A, Wang Z, Kishi M, Frank E, Sanes JR (2014) SAD kinases control the maturation of nerve terminals in the mammalian peripheral and central nervous systems. Proc Natl Acad Sci 111:1138-1143.

Lindsey BG (1975) Fine structure and distribution of axon terminals from the cochlear nucleus on neurons in the medial superior olivary nucleus of the cat. J Comp Neurol 160:81-103.

Lipschutz JH, Mostov KE (2002) Exocytosis: the many masters of the exocyst. Curr Biol 12:R212-4.

Liu SJ, Kaczmarek LK (1998a) The expression of two splice variants of the Kv3.1 potassium channel gene is regulated by different signaling pathways. J Neurosci 18:2881-2890.

Liu SJ, Kaczmarek LK (1998b) Depolarization selectively increases the expression of the Kv3.1 potassium channel in developing inferior colliculus neurons. J Neurosci 18:8758-8769.

Lomeli H, Mosbacher J, Melcher T, Höger T, Jörg RP, Kuner T, Monyer H, Higuchi M, Bach A, Seeburg PH (1994) Control of Kinetic Properties of AMPA Receptor Channels by Nuclear RNA Editing. Science (80- ) 266:1709-1713.

Luo L, O'Leary DDM (2005) Axon retraction and degeneration in development and disease. 
Annu Rev Neurosci 28:127-156.

Lynall MM-E, Bassett DS, Kerwin R, McKenna PJ, Kitzbichler M, Muller U, Bullmore E (2010) Functional connectivity and brain networks in schizophrenia. J Neurosci 30:9477-9487.

Macica CM, von Hehn CAA, Wang L-Y, Ho C-S, Yokoyama S, Joho RH, Kaczmarek LK (2003) Modulation of the Kv3.1b potassium channel isoform adjusts the fidelity of the firing pattern of auditory neurons. J Neurosci 23:1133-1141.

Maricich SM, Xia A, Mathes EL, Wang VY, Oghalai JS, Fritzsch B, Zoghbi HY (2009) Atoh1Lineal Neurons Are Required for Hearing and for the Survival of Neurons in the Spiral Ganglion and Brainstem Accessory Auditory Nuclei. J Neurosci 29:11123-11133.

Marrs GS, Morgan WJ, Howell DM, Spirou GA, Mathers PH (2013) Embryonic origins of the mouse superior olivary complex. Dev Neurobiol 73:384-398.

Marrs GS, Spirou GA (2012) Embryonic assembly of auditory circuits: spiral ganglion and brainstem. J Physiol 590:2391-2408.

Martin MR, Rickets C (1981) Histogenesis of the cochlear nucleus of the mouse. J Comp Neurol 197:169-184.

Masterton B, Diamond IT, Harrison J, Beecher M (1967) Medial Superior Olive and Sound Localization. Science (80- ) 155:1696-1697.

Mattox D, GR N, Gulley R (1982) A freeze-fracture study of the maturation of synapses in the anteroventral cochlear nucleus of the developing rat. Anat Rec 204:281-287.

Mayer M, Westbrook G, Guthrie P (1984) Voltage-dependent block by Mg2+ of NMDA responses in spinal cord neurones. Nature 309:261-263.

Mencía A, Modamio-Høybjør S, Redshaw N, Morín M, Mayo-Merino F, Olavarrieta L, Aguirre LA, Del Castillo I, Steel KP, Dalmay T, Moreno F, Moreno-Pelayo MA (2009) Mutations in the seed region of human miR-96 are responsible for nonsyndromic progressive hearing loss. Nat Genet 41:609-613.

Michalski N, Babai N, Renier N, Perkel DJ, Chédotal A, Schneggenburger R (2013) Robo3driven axon midline crossing conditions functional maturation of a large commissural synapse. Neuron 78:855-868.

Mittelstaedt T, Alvaréz-Baron E, Schoch S (2010) RIM proteins and their role in synapse function. Biol Chem 391:599-606.

Mizutani H, Hori T, Takahashi T (2006) 5-HT1B receptor-mediated presynaptic inhibition at the calyx of Held of immature rats. Eur J Neurosci 24:1946-1954.

Montcouquiol M, Sans N, Huss D, Kach J, Dickman JD, Forge A, Rachel RA, Copeland NG, Jenkins NA, Bogani D, Murdoch J, Warchol ME, Wenthold RJ, Kelley MW (2006) Asymmetric Localization of Vangl2 and Fz3 Indicate Novel Mechanisms for Planar Cell Polarity in Mammals. J Neurosci 26:5265-5275.

Moore MJ, Caspary DM (1983) Strychnine blocks binaural inhibition in lateral superior olivary neurons. J Neurosci 3:237-242.

Morara S, Van Der Want JJL, De Weerd H, Provinp L, Rosina A (2001) Ultrastructural analysis of climbing fiber-Purkinje cell synaptogenesis in the rat cerebellum. Neuroscience 108:655-671. 
Morest D (1968a) The growth of synaptic endings in the mammalian brain: a study of the calyces of the trapezoid body. Anat Embryol (Berl) 127:201-220.

Morest $D(1968 b)$ The collateral system of the medial nucleus of the trapezoid body of the cat, its neuronal architecture and relation to the olivo-cochlear bundle. Brain Res 9:288-311.

Mugnaini E, Osen KK, Dahl AL, Friedrich VL, Korte G (1980) Fine structure of granule cells and related interneurons (termed Golgi cells) in the cochlear nuclear complex of cat, rat and mouse. J Neurocytol 9:537-570.

Müller J, Reyes-Haro D, Pivneva T, Nolte C, Schaette R, Lübke J, Kettenmann H (2009) The principal neurons of the medial nucleus of the trapezoid body and NG2(+) glial cells receive coordinated excitatory synaptic input. J Gen Physiol 134:115-127.

Muniak MA, Rivas A, Montey KL, May BJ, Francis HW, Ryugo DK (2013) 3D model of frequency representation in the cochlear nucleus of the CBA/J mouse. J Comp Neurol 521:1510-1532.

Muniak MA, Ryugo DK (2014) Tonotopic organization of vertical cells in the dorsal cochlear nucleus of the CBA/J mouse. J Comp Neurol 522:937-949.

Murthy M, Garza D, Scheller RH, Schwarz TL (2003) Mutations in the exocyst component Sec5 disrupt neuronal membrane traffic, but neurotransmitter release persists. Neuron 37:433447.

Nagy B, Hovhannisyan A, Barzan R, Chen TJ, Kukley M (2017) Different patterns of neuronal activity trigger distinct responses of oligodendrocyte precursor cells in the corpus callosum. PLoS Biol 15:1-32.

Nakamura PA, Hsieh CY, Cramer KS (2012) EphB signaling regulates target innervation in the developing and deafferented auditory brainstem. Dev Neurobiol 72:1243-1255.

Nakamura Y, Harada H, Kamasawa N, Matsui K, Rothman JS, Shigemoto R, Silver RA, DiGregorio DA, Takahashi T (2015) Nanoscale Distribution of Presynaptic Ca2+Channels and Its Impact on Vesicular Release during Development. Neuron 85:145-159.

Nakamura Y, Takahashi T (2007) Developmental changes in potassium currents at the rat calyx of Held presynaptic terminal. J Physiol 581:1101-1112.

Nanou E, Catterall WA (2018) Calcium Channels, Synaptic Plasticity, and Neuropsychiatric Disease. Neuron 98:466-481.

Nawabi H, Castellani V (2011) Axonal commissures in the central nervous system: How to cross the midline? Cell Mol Life Sci 68:2539-2553.

Nichols DH, Bruce LL (2006) Migratory routes and fates of cells transcribing the Wnt-1 gene in the murine hindbrain. Dev Dyn 235:285-300.

Nieto M, Bradley L, Hunt P, Das Gupta R, Krumlauf R, Wilkinson D (1992) Molecular mechanisms of pattern formation in the vertebrate hindbrain. Ciba Found Symp 165:92107.

Noctor SC, Martínez-Cerdeño V, Ivic L, Kriegstein AR (2004) Cortical neurons arise in symmetric and asymmetric division zones and migrate through specific phases. Nat Neurosci 7:136-144.

O'Connor-Giles KM, Ho LL, Ganetzky B (2008) Nervous Wreck Interacts with Thickveins and 
the Endocytic Machinery to Attenuate Retrograde BMP Signaling during Synaptic Growth. Neuron 58:507-518.

O'Donnell WT, Warren ST (2002) A Decade of Molecular Studies of Fragile X Syndrome. Annu Rev Neurosci 25:315-338.

Oertel D, Wright S, Cao XJ, Ferragamo M, Bal R (2011) The multiple functions of T stellate/multipolar/chopper cells in the ventral cochlear nucleus. Hear Res 276:61-69.

Ohtsuki G, Hirano T (2008) Bidirectional plasticity at developing climbing fiber-Purkinje neuron synapses. Eur J Neurosci 28:2393-2400.

Ollo C, Schwartz IR (1979) The superior olivary complex in C57BL/6 mice. Am J Anat.

Osen KK (1969) Cytoarchitecture of the Cochlear Nuclei in the Cat. J Comp Neurol 136:453484.

Parnavelas JG (1999) Glial cell lineages in the rat cerebral cortex. Exp Neurol 156:418-429.

Pasic TR, Moore DR, Rubel EW (1994) Effect of altered neuronal activity on cell size in the medial nucleus of the trapezoid body and ventral cochlear nucleus of the gerbil. J Comp Neurol 348:111-120.

Paxinos G, Xu-Feng H, Sengul G, Watson C (2012) Organization of Brainstem Nuclei. In: The Human Nervous System, 3rd ed. (Mai J, Paxinos G, eds), pp 260-327. London: Elsevier Inc.

Pecka M, Brand A, Behrend O, Grothe B (2008) Interaural Time Difference Processing in the Mammalian Medial Superior Olive: The Role of Glycinergic Inhibition. J Neurosci 28:69146925.

Perkins GA, Tjong J, Brown JM, Poquiz PH, Scott RT, Kolson DR, Ellisman MH, Spirou GA (2010) The micro-architecture of mitochondria at active zones: electron tomography reveals novel anchoring scaffolds and cristae structured for high-rate metabolism. J Neurosci 30:1015-1026.

Perney TM, Marshall J, Martin K a, Hockfield S, Kaczmarek LK (1992) Expression of the mRNAs for the Kv3.1 potassium channel gene in the adult and developing rat brain. $\mathrm{J}$ Neurophysiol 68:756-766.

Peters A, Palay S, Webster H (1991) The Fine Structure of the Nervous System: Neurons and Their Supporting Cells, 3rd ed. USA: Oxford University Press.

Pfenninger KH, Laurino L, Peretti D, Wang X, Rosso S, Morfini G, Caceres A, Quiroga S (2003) Regulation of membrane expansion at the nerve growth cone. J Cell Sci 116:1209-1217.

Pierce E (1973) Time of origin of neurons in the brain stem of the mouse. Prog Brain Res 40:53-65.

Pierce ET (1967) Histogenesis of the dorsal and ventral cochlear nuclei in the mouse. An autoradiographic study. J Comp Neurol 131:27-53.

Pilz DT, Matsumoto N, Minnerath S, Mills P, Gleeson JG, Allen KM, Walsh C a, Barkovich a J, Dobyns WB, Ledbetter DH, Ross ME (1998) LIS1 and XLIS (DCX) mutations cause most classical lissencephaly, but different patterns of malformation. Hum Mol Genet 7:20292037. 
Polgar N, Fogelgren B (2017) Regulation of Cell Polarity by Exocyst-Mediated Trafficking. Cold Spring Harb Perspect Biol.

Pologruto TA, Sabatini BL, Svoboda K (2003) ScanImage: flexible software for operating laser scanning microscopes. Biomed Eng Online 2:13.

Purves D, Lichtman J (1980) Elimination of synapses in the developing nervous system. Science (80- ) 210:153-157.

Queisser G, Wiegert S, Bading H (2011) Structural dynamics of the cell nucleus. Nucleus 2:98104.

Ramón y Cajal S (1909) Histologie du Systeme Nerveux de l'Homme et des Vertebres. Maloine, Paris: 1911. chap. II. Demography v.1:3-43.

Rayleigh, Lord (1907) On our perception of sound direction. Philos Mag Ser 6 13:214-232.

Razafsky D, Blecher N, Markov A, Stewart-Hutchinson PJ, Hodzic D (2012) LINC complexes mediate the positioning of cone photoreceptor nuclei in mouse retina. PLoS One 7:e47180.

Razafsky D, Hodzic D (2009) Bringing KASH under the SUN: the many faces of nucleocytoskeletal connections. J Cell Biol 186:461-472.

Razafsky D, Hodzic D (2014) Temporal and tissue-specific disruption of LINC complexes in vivo. Genesis 52:359-365.

Reiner O, Carrozzo R, Shen Y, Wehnert M, Faustinella F, Dobyns WB, Caskey CT, Ledbetter $\mathrm{DH}$ (1993) Isolation of a Miller-Dieker lissencephaly gene containing $\mathrm{G}$ protein $\beta$-subunitlike repeats. Nature 364:717-721.

Renden R (2005) Glutamate Transporter Studies Reveal the Pruning of Metabotropic Glutamate Receptors and Absence of AMPA Receptor Desensitization at Mature Calyx of Held Synapses. J Neurosci 25:8482-8497.

Renier N, Schonewille M, Giraudet F, Badura A, Tessier-Lavigne M, Avan P, De Zeeuw Cl, Chédotal A (2010) Genetic dissection of the function of hindbrain axonal commissures. PLoS Biol 8:e1000325.

Reyes-Haro D, Müller J, Boresch M, Pivneva T, Benedetti B, Scheller A, Nolte C, Kettenmann H (2010) Neuron-astrocyte interactions in the medial nucleus of the trapezoid body. J Gen Physiol 135:583-594.

Rice D, Barone S (2000) Critical periods of vulnerability for the developing nervous system: Evidence from humans and animal models. Environ Health Perspect 108:511-533.

Rodríguez-Contreras A, de Lange RPJ, Lucassen PJ, Borst JGG (2006) Branching of calyceal afferents during postnatal development in the rat auditory brainstem. J Comp Neurol 496:214-228.

Rodríguez-Contreras A, van Hoeve JSS, Habets RLP, Locher H, Borst JGG (2008) Dynamic development of the calyx of Held synapse. Proc Natl Acad Sci 105:5603-5608.

Romand R, Ehret G (1990) Development of tonotopy in the inferior colliculus. I. Electrophysiological mapping in house mice. Brain Res Dev Brain Res 54:221-234.

Rosengauer E, Hartwich H, Hartmann AM, Rudnicki A, Satheesh SV, Avraham KB, Nothwang HG (2012) Egr2::Cre-mediated conditional ablation of Dicer disrupts histogenesis of 
mammalian central auditory nuclei. PLoS One 7:e49503.

Rotschafer SE, Marshak S, Cramer KS (2015) Deletion of Fmr1 alters function and synaptic inputs in the auditory brainstem. PLoS One 10:1-15.

Rowland K, Irby N, Spirou G (2000) Specialized synapse-associated structures within the calyx of Held. J Neurosci 20:9135-9144.

Rusu SI, Borst JGG (2011) Developmental changes in intrinsic excitability of principal neurons in the rat medial nucleus of the trapezoid body. Dev Neurobiol 71:284-295.

Ryugo DK (2008) Projections of low spontaneous rate, high threshold auditory nerve fibers to the small cell cap of the cochlear nucleus in cats. Neuroscience 154:114-126.

Ryugo DK, Haenggeli CA, Doucet JR (2003) Multimodal inputs to the granule cell domain of the cochlear nucleus. Exp Brain Res 153:477-485.

Ryugo DK, Spirou GA (2010) Auditory System: Giant Synaptic Terminals, Endbulbs, and Calyces. Encycl Neurosci 1:759-770.

Sabatier C, Plump AS, Ma L, Brose K, Tamada A, Murakami F, Lee EYHP, Tessier-Lavigne M (2004) The divergent Robo family protein Rig-1/Robo3 is a negative regulator of Slit responsiveness required for midline crossing by commissural axons. Cell 117:157-169.

Saliu A, Adise S, Xian S, Kudelska K, Rodríguez-Contreras A (2014) Natural and lesion-induced decrease in cell proliferation in the medial nucleus of the trapezoid body during hearing development. J Comp Neurol 522:971-985.

Sätzler K et al. (2002) Three-dimensional reconstruction of a calyx of Held and its postsynaptic principal neuron in the medial nucleus of the trapezoid body. J Neurosci 22:10567-10579.

Schecterson LC, Sanchez JT, Rubel EW, Bothwell M (2012) TrkB Downregulation Is Required for Dendrite Retraction in Developing Neurons of Chicken Nucleus Magnocellularis. J Neurosci 32:14000-14009.

Schlüter T, Berger C, Rosengauer E, Fieth P, Krohs C, Ushakov K, Steel KP, Avraham KB, Hartmann AK, Felmy F, Nothwang HG (2018) miR-96 is required for normal development of the auditory hindbrain. Hum Mol Genet 27:860-874.

Schneggenburger R, Forsythe I (2006) The calyx of Held. Cell Tissue Res 326:311-337.

Schwenger DB, Kuner T (2010) Acute genetic perturbation of exocyst function in the rat calyx of Held impedes structural maturation, but spares synaptic transmission. Eur J Neurosci 32:974-984.

Semple B, Blomgren K, Gimlin K, Ferriero D, Noble-Haeusslein L (2013) Brain development in rodents and humans: Identifying benchmarks of maturation and vulnerability to injury across species. Prog Neurobiol 106-107:1-16.

Seung S (2012) Connectome: How the Brain's Wiring Makes Us Who We Are, 1st ed. Boston, MA: Houghton Mifflin Harcourt.

Shelly M, Poo MM (2011) Role of LKB1-SAD/MARK pathway in neuronal polarization. Dev Neurobiol 71:508-527.

Shen L, Lin Y, Sun Z, Yuan X, Chen L, Shen B (2016) Knowledge-Guided Bioinformatics Model for Identifying Autism Spectrum Disorder Diagnostic MicroRNA Biomarkers. Sci Rep 6:1-9. 
Shi S, Jan L, Jan Y (2003) Hippocampal neuronal polarity specified by spatially localized $\mathrm{mPar3/mPar6}$ and PI 3-kinase activity. Cell 112:63-75.

Sierksma MC, Tedja MS, Borst JGG (2017) In vivo matching of postsynaptic excitability with spontaneous synaptic inputs during formation of the rat calyx of Held synapse. J Physiol 595:207-231.

Sinclair JL, Fischl MJ, Alexandrova O, Heß M, Grothe B, Leibold C, Kopp-Scheinpflug C (2017) Sound-evoked activity influences myelination of brainstem axons in the trapezoid body. $J$ Neurosci 37:3728-16.

Singh M, Denny H, Smith C, Granados J, Renden R (2018) Presynaptic loss of dynamin related protein 1 impairs synaptic vesicle release and recycling at the mouse calyx of held. $\mathrm{J}$ Physiol:1-72.

Smith P, Joris P, Carney L, Yin T (1991) Projections of physiologically characterized globular bushy cell axons from the cochlear nucleus of the cat. J Comp Neurol 304:387-407.

Smith PH, Joris PX, Yin TCT (1993) Projections of physiologically characterized spherical bushy cell axons from the cochlear nucleus of the cat: Evidence for delay lines to the medial superior olive. J Comp Neurol 331:245-260.

Smith PH, Rhode WS (1989) Structural and Functional Properties Distinguish Two Types of Multipolar Cells in the Ventral Cochlear Nucleus. J Comp Neurol 282:595-616.

Sommer B, Keinanen K, Verdoorn TA, Wisden W, Burnashev N, Herb A, Kohler M, Takagi T, Sakmann B, Seeburg PH (1990) Flip and Flop: A Cell-Specific Functional Switch in Glutamate-Operated Channels of the CNS. Science (80- ) 249:1580-1585.

Song I, Huganir R (2002) Regulation of AMPA receptors during synaptic plasticity. Trends Neurosci 25:578-588.

Song P, Yang Y, Barnes-Davies M, Bhattacharjee A, Hamann M, Forsythe ID, Oliver DL, Kaczmarek LK (2005) Acoustic environment determines phosphorylation state of the Kv3.1 potassium channel in auditory neurons. Nat Neurosci 8:1335-1342.

Sonntag M, Englitz B, Kopp-Scheinpflug C, Rübsamen R (2009) Early postnatal development of spontaneous and acoustically evoked discharge activity of principal cells of the medial nucleus of the trapezoid body: an in vivo study in mice. J Neurosci 29:9510-9520.

Sonntag M, Englitz B, Typlt M, Rübsamen R (2011) The calyx of held develops adult-like dynamics and reliability by hearing onset in the mouse in vivo. J Neurosci 31:6699-6709.

Soria Van Hoeve JS, Borst JGG (2010) Delayed appearance of the scaffolding proteins PSD-95 and Homer-1 at the developing rat calyx of held synapse. J Comp Neurol 518:4581-4590.

Spirou G, Brownell W, Zidanic M (1990) Recordings from cat trapezoid body and HRP labeling of globular bushy cell axons. J Neurophysiol 63:1169-1190.

Spirou G, May B, Wright D, Ryugo D (1993) Frequency organization of the dorsal cochlear nucleus in cats. J Comp Neurol 329:36-52.

Spirou GAG, Rowland KKC, Berrebi AS (1998) Ultrastructure of neurons and large synaptic terminals in the lateral nucleus of the trapezoid body of the cat. J Comp Neurol 398:257272.

Steinert JR, Postlethwaite M, Jordan MD, Chernova T, Robinson SW, Forsythe ID (2010) 
NMDAR-mediated EPSCs are maintained and accelerate in time course during maturation of mouse and rat auditory brainstem in vitro. J Physiol 588:447-463.

Stotler WA (1953) An experimental study of the cells and connections of the superior olivary complex of the cat. J Comp Neurol 98:401-431.

Strumbos JG, Brown MR, Kronengold J, Polley DB, Kaczmarek LK (2010) Fragile X Mental Retardation Protein Is Required for Rapid Experience-Dependent Regulation of the Potassium Channel Kv3.1b. J Neurosci 30:10263-10271.

Sudarov A, Gooden F, Tseng D, Gan WB, Ross ME (2013) Lis1 controls dynamics of neuronal filopodia and spines to impact synaptogenesis and social behaviour. EMBO Mol Med 5:591-607.

Sugihara I (2005) Microzonal projection and climbing fiber remodeling in single olivocerebellar axons of newborn rats at postnatal days 4-7. J Comp Neurol 487:93-106.

Supekar K, Uddin LLQ, Khouzam A, Phillips J, Gaillard WD, Kenworthy LE, Yerys BE, Vaidya CJ, Menon V (2013) Brain hyperconnectivity in children with autism and its links to social deficits. Cell Rep 5:738-747.

Takahashi H, Craig AM (2013) Protein tyrosine phosphatases PTPס, PTPs, and LAR: Presynaptic hubs for synapse organization. Trends Neurosci 36:522-534.

Takahashi T, Kajikawa Y, Tsujimoto T (1998) G-Protein-coupled modulation of presynaptic calcium currents and transmitter release by a GABAB receptor. J Neurosci 18:3138-3146.

Tam PPL, Behringer RR (1997) Mouse gastrulation: The formation of a mammalian body plan. Mech Dev 68:3-25.

Tapia JC, Wylie JD, Kasthuri N, Hayworth KJ, Schalek R, Berger DR, Guatimosim C, Seung HS, Lichtman JW (2012) Pervasive Synaptic Branch Removal in the Mammalian Neuromuscular System at Birth. Neuron 74:816-829.

Taschenberger H, Leão RM, Rowland KC, Spirou GA, von Gersdorff H (2002) Optimizing Synaptic Architecture and Efficiency for High-Frequency Transmission. Neuron 36:11271143.

Taschenberger H, von Gersdorff H (2000) Fine-tuning an auditory synapse for speed and fidelity: developmental changes in presynaptic waveform, EPSC kinetics, and synaptic plasticity. J Neurosci 20:9162-9173.

Teodoro RO, Pekkurnaz G, Nasser A, Higashi-Kovtun ME, Balakireva M, Mclachlan IG, Camonis J, Schwarz TL (2013) Ral mediates activity-dependent growth of postsynaptic membranes via recruitment of the exocyst. EMBO J 32:2039-2055.

Thakar S, Wang L, Yu T, Ye M, Onishi K, Scott J, Qi J, Fernandes C, Han X, Yates JR, Berg DK, Zou Y (2017) Evidence for opposing roles of Celsr3 and Vangl2 in glutamatergic synapse formation. Proc Natl Acad Sci 114:E610-E618.

Theodosis DT, Poulain DA, Oliet SHR (2008) Activity-Dependent Structural and Functional Plasticity of Astrocyte-Neuron Interactions. Physiol Rev 88:983-1008.

Thompson SP (1882) On the function of the two ears in the perception of space. Philos Mag Ser 5 13:406-416.

Tomchek SD, Dunn W (2007) Sensory Processing in Children With and Without Autism: a 
Comparative Study Using the Short Sensory Profile. Am J Occup Ther 61:190-200.

Toyoshima M, Sakurai K, Shimazaki K, Takeda Y, Nakamoto M, Serizawa S, Shimoda Y, Watanabe K (2009a) Preferential localization of neural cell recognition molecule NB-2 in developing glutamatergic neurons in the rat auditory brainstem. J Comp Neurol 513:349_ 362.

Toyoshima M, Sakurai K, Shimazaki K, Takeda Y, Shimoda Y, Watanabe K (2009b) Deficiency of neural recognition molecule NB-2 affects the development of glutamatergic auditory pathways from the ventral cochlear nucleus to the superior olivary complex in mouse. Dev Biol 336:192-200.

Traynelis S, Wollmuth L, McBain C, Menniti F, Vance K, Ogden K, Hansen K, Yuan H, Myers S, Dingledine R (2010) Glutamate Receptor Ion Channels: Structure, Regulation, and Function. Pharmacol Rev 62:405-496.

Tritsch NX, Bergles DE (2010) Developmental regulation of spontaneous activity in the Mammalian cochlea. J Neurosci 30:1539-1550.

Tritsch NX, Rodríguez-Contreras A, Crins TTH, Wang HC, Borst JGG, Bergles DE (2010) Calcium action potentials in hair cells pattern auditory neuron activity before hearing onset. Nat Neurosci 13:1050-1052.

Tritsch NX, Yi E, Gale JE, Glowatzki E, Bergles DE (2007) The origin of spontaneous activity in the developing auditory system. Nature 450:50-55.

Turecek R, Trussell LO (2001) Presynaptic glycine receptors enhance transmitter release at a mammalian central synapse. Nature 411:587-590.

Turecek R, Trussell LO (2002) Reciprocal developmental regulation of presynaptic ionotropic receptors. Proc Natl Acad Sci 99:13884-13889.

Turney SG, Lichtman JW (2012) Reversing the outcome of synapse elimination at developing neuromuscular junctions in vivo: evidence for synaptic competition and its mechanism. PLoS Biol 10:e1001352.

Uwechue NM, Marx M-C, Chevy Q, Billups B (2012) Activation of glutamate transport evokes rapid glutamine release from perisynaptic astrocytes. J Physiol 590:2317-2331.

Van Hartesveldt C, Moore B, Hartman BK (1986) Transient midline raphe glial structure in the developing rat. J Comp Neurol 253:175-184.

Vaughn J (1989) Fine structure of synaptogenesis in the vertebrate central nervous system. Synapse 3:255-285.

von Gersdorff H, Borst JGG (2002) Short-term plasticity at the calyx of Held. Nat Rev Neurosci 3:53-64.

von Hehn CAA, Bhattacharjee A, Kaczmarek LK (2004) Loss of Kv3.1 Tonotopicity and Alterations in cAMP Response Element-Binding Protein Signaling in Central Auditory Neurons of Hearing Impaired Mice. J Neurosci 24:1936-1940.

Walsh MK, Lichtman JW (2003) In vivo time-lapse imaging of synaptic takeover associated with naturally occurring synapse elimination. Neuron 37:67-73.

Wang LY, Gan L, Forsythe ID, Kaczmarek LK (1998) Contribution of the Kv3.1 potassium channel to high-frequency firing in mouse auditory neurones. J Physiol 509:183-194. 
Wang T, de Kok L, Willemsen R, Elgersma Y, Borst JGG (2015) In vivo synaptic transmission and morphology in mouse models of Tuberous sclerosis, Fragile $X$ syndrome, Neurofibromatosis type 1, and Costello syndrome. Front Cell Neurosci 9:1-15.

Wang VY, Rose MF, Zoghbi HY (2005) Math1 expression redefines the rhombic lip derivatives and reveals novel lineages within the brainstem and cerebellum. Neuron 48:31-43.

Warr W (1966) Fiber Degeneration Following Lesions in the Anterior Ventral Cochlear Nucleus of the Cat. Exp Neurol 14:453-474.

Warr W (1972) Fiber degeneration following lesions in the multipolar and globular cell areas in the ventral cochlear nucleus of the cat. Brain Res 40:247-270.

Watanabe M, Kano M (2011) Climbing fiber synapse elimination in cerebellar Purkinje cells. Eur J Neurosci 34:1697-1710.

Weatherstone JH, Kopp-Scheinpflug C, Pilati N, Wang Y, Forsythe ID, Rubel EW, Tempel BL (2016) Maintenance of neuronal size gradient in MNTB requires sound-evoked activity. J Neurophysiol 117:jn.00528.2016.

Webster DB (1983) Auditory neuronal sizes after a unilateral conductive hearing loss. Exp Neurol 79:130-140.

Webster DB, Trune DR (1982) Cochlear nuclear complex of mice. Am J Anat 163:103-130.

Weedman DL, Ryugo DK (1996) Projections from auditory cortex to the cochlear nucleus in rats: synapses on granule cell dendrites. J Comp Neurol 371:311-324.

Wiggin GR, Fawcett JP, Pawson T (2005) Polarity proteins in axon specification and synaptogenesis. Dev Cell 8:803-816.

Wilke SA, Antonios JK, Bushong EA, Badkoobehi A, Malek E, Hwang M, Terada M, Ellisman $\mathrm{MH}$, Ghosh A (2013) Deconstructing complexity: serial block-face electron microscopic analysis of the hippocampal mossy fiber synapse. J Neurosci 33:507-522.

Wimmer V, Nevian T, Kuner T (2004) Targeted in vivo expression of proteins in the calyx of Held. Pflugers Arch Eur J Physiol 449:319-333.

Wimmer VC, Horstmann H, Groh A, Kuner T (2006) Donut-Like Topology of Synaptic Vesicles with a Central Cluster of Mitochondria Wrapped into Membrane Protrusions: A Novel Structure-Function Module of the Adult Calyx of Held. J Neurosci 26:109-116.

Wingate RJT (2001) The rhombic lip and early cerebellar development. Curr Opin Neurobiol $11: 82-88$.

Wittmann M, Queisser G, Eder A, Wiegert JS, Bengtson CP, Hellwig A, Wittum G, Bading H (2009) Synaptic Activity Induces Dramatic Changes in the Geometry of the Cell Nucleus: Interplay between Nuclear Structure, Histone H3 Phosphorylation, and Nuclear Calcium Signaling. J Neurosci 29:14687-14700.

Wu H, Xiong WC, Mei L (2010) To build a synapse: signaling pathways in neuromuscular junction assembly. Development 137:1017-1033.

Xiang L, Chen X-J, Wu K-C, Zhang C-J, Zhou G-H, Lv J-N, Sun L-F, Cheng F-F, Cai X-B, Jin Z$B(2017)$ miR-183/96 plays a pivotal regulatory role in mouse photoreceptor maturation and maintenance. Proc Natl Acad Sci 114:6376-6381. 
Xiao L, Michalski N, Kronander E, Gjoni E, Genoud C, Knott G, Schneggenburger R (2013) BMP signaling specifies the development of a large and fast CNS synapse. Nat Neurosci 16:1-11.

Xu J, McNeil B, Wu W, Nees D, Bai L, Wu LG (2008) GTP-independent rapid and slow endocytosis at a central synapse. Nat Neurosci 11:45-53.

Yamashita T, Eguchi K, Saitoh N, von Gersdorff H, Takahashi T (2010) Developmental shift to a mechanism of synaptic vesicle endocytosis requiring nanodomain $\mathrm{Ca} 2+$. Nat Neurosci 13:838-844.

Yang S, Adhikari S, Dobbala M, Adusumilli S, Rowley JD, Ganikhanov F, Zhang L, Marrs G, Wysolmerski R, Spirou GA (2013) Multi-color ultrafast laser platform for nonlinear optical imaging based on independently tunable optical parametric oscillators. Appl Phys B 111:617-625.

Yang Y-M, Aitoubah J, Lauer A, Nuriya M, Takamiya K, Jia Z, May B, Huganir R, Wang L-Y (2011) GluA4 is indispensable for driving fast neurotransmission across a high-fidelity central synapse. J Physiol 589:4209-4227.

Yang Y-M, Wang L-Y (2006) Amplitude and Kinetics of Action Potential-Evoked Ca2+ Current and Its Efficacy in Triggering Transmitter Release at the Developing Calyx of Held Synapse. J Neurosci 26:5698-5708.

Yang YM, Fedchyshyn MJ, Grande G, Aitoubah J, Tsang CW, Xie H, Ackerley CA, Trimble WS, Wang LY (2010) Septins regulate developmental switching from microdomain to nanodomain coupling of $\mathrm{Ca} 2+$ influx to neurotransmitter release at a central synapse. Neuron 67:100-115.

Yates N, Robertson D, Martin-Iverson M, Rodger J (2014) Auditory brainstem responses of ephrin-A2, ephrin-A5(-/-) and ephrin-A2A5(-/-) mice. Audiol Neurootol 19:115-126.

Ye Y, Machado DG, Kim DO (2000) Projection of the marginal shell of the anteroventral cochlear nucleus to olivocochlear neurons in the cat. J Comp Neurol 420:127-138.

Yeckel MF, Sleeper AA, Fitzpatrick JS, Hertle DN, Hagenston AM, Garner RT (2008) Intracellular calcium waves transmit synaptic information to the nucleus in hippocampal pyramidal neurons. Transcr Regul by Neuronal Act To Nucl Back:73-89.

Yoshioka T, Hagiwara A, Hida Y, Ohtsuka T (2013) Vangl2, the planar cell polarity protein, is complexed with postsynaptic density protein PSD-95. FEBS Lett 587:1453-1459.

Youssoufian M, Couchman K, Shivdasani MN, Paolini AG, Walmsley B (2008) Maturation of auditory brainstem projections and calyces in the congenitally deaf $(\mathrm{dn} / \mathrm{dn})$ mouse. J Comp Neurol 506:442-451.

Yuan X, Eisen AM, McBain CJH, Gallo V (1998) A role for glutamate and its receptors in the regulation of oligodendrocyte development in cerebellar tissue slices. Development 125:2901-2914.

Zajac A, Sun X, Zhang J, Guo W (2005) Cyclical Regulation of the Exocyst and Cell Polarity Determinants for Polarized Cell Growth. Mol Biol Cell 16:1500-1512.

Zarnescu DC, Jin P, Betschinger J, Nakamoto M, Wang Y, Dockendorff TC, Feng Y, Jongens TA, Sisson JC, Knoblich JA, Warren ST, Moses K (2005) Fragile X protein functions with $\mathrm{Lgl}$ and the PAR complex in flies and mice. Dev Cell 8:43-52. 
Zelina P, Blockus H, Zagar Y, Péres A, Friocourt F, Wu Z, Rama N, Fouquet C, Hohenester E, Tessier-Lavigne M, Schweitzer J, Crollius HR, Chédotal A (2014) Signaling switch of the axon guidance receptor Robo3 during vertebrate evolution. Neuron 84:1258-1272.

Zhang B, Seigneur E, Wei P, Gokce O, Morgan J, Südhof TC (2016) Developmental plasticity shapes synaptic phenotypes of autism-associated neuroligin-3 mutations in the calyx of Held. Mol Psychiatry 22:1483-1491.

Zhang KD, Stoller ML, Fekete DM (2015) Expression and misexpression of the miR-183 family in the developing hearing organ of the chicken. PLoS One 10:1-22.

Zuko A, Kleijer KTE, Oguro-Ando A, Kas MJH, Van Daalen E, Van Der Zwaag B, Burbach JPH (2013) Contactins in the neurobiology of autism. Eur J Pharmacol 719:63-74.

\section{Figures}

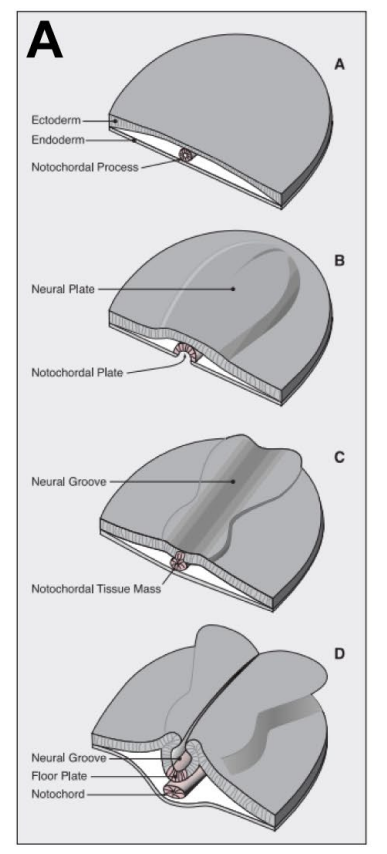

B
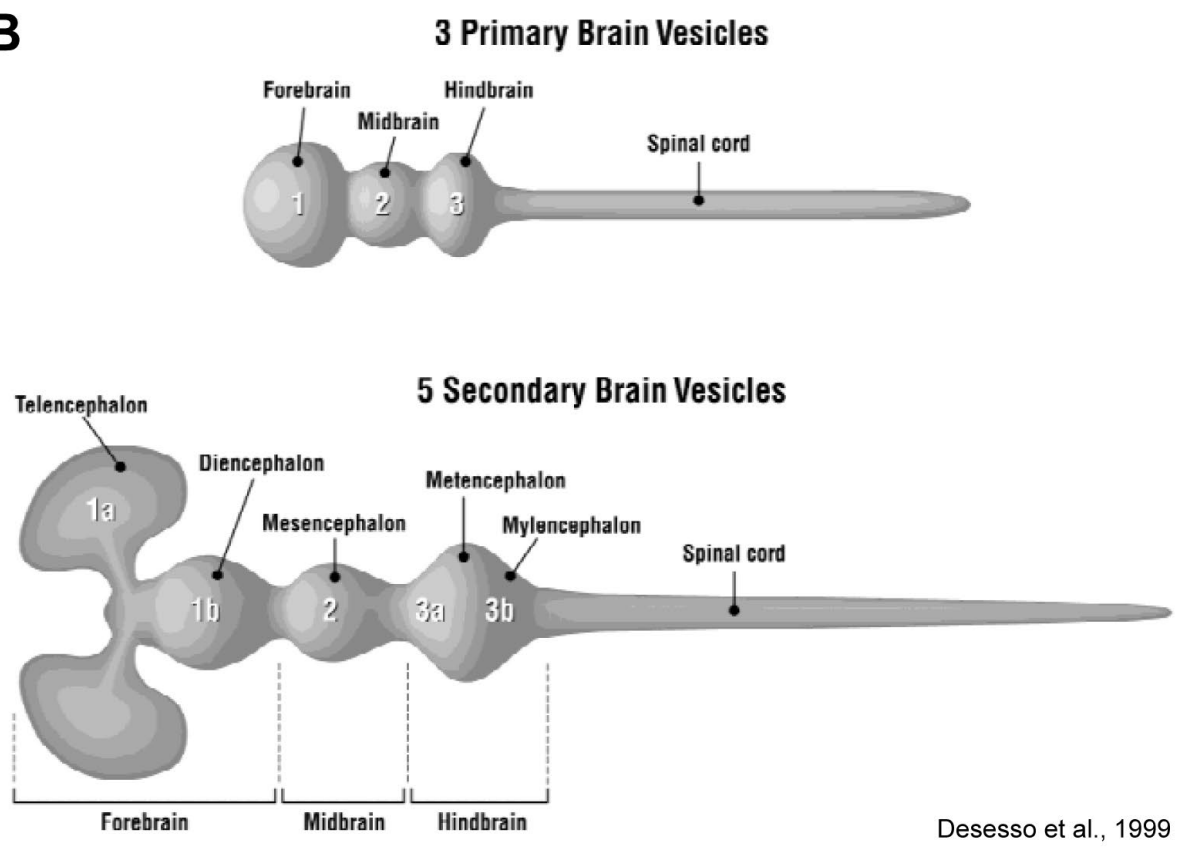

Figure 1: Development of the Nervous System. A. Early in development, multiple infoldings of the layered embryo occur to form the neural groove, which will close to form the neural tube-the basis of the developing nervous system. B. As development proceeds, the neural tube subdivides into three distinct brain vescicles (forebrain, midbrain, hindbrain). The forebrain further subdivides into the telencephalon and diencephalon, while the hindbrain subdivides into the metencephalon and mylencephalon. Adapted from Desesso et al, 1999. 
A

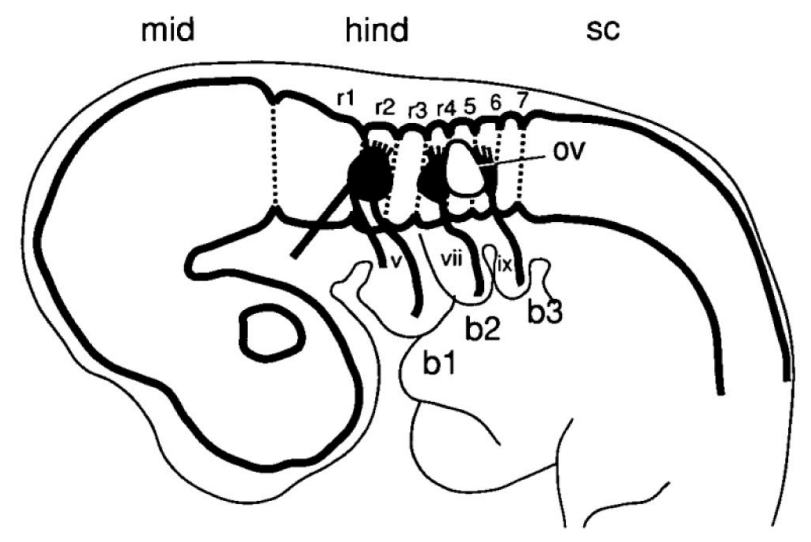

Hunt et al., 1991

B

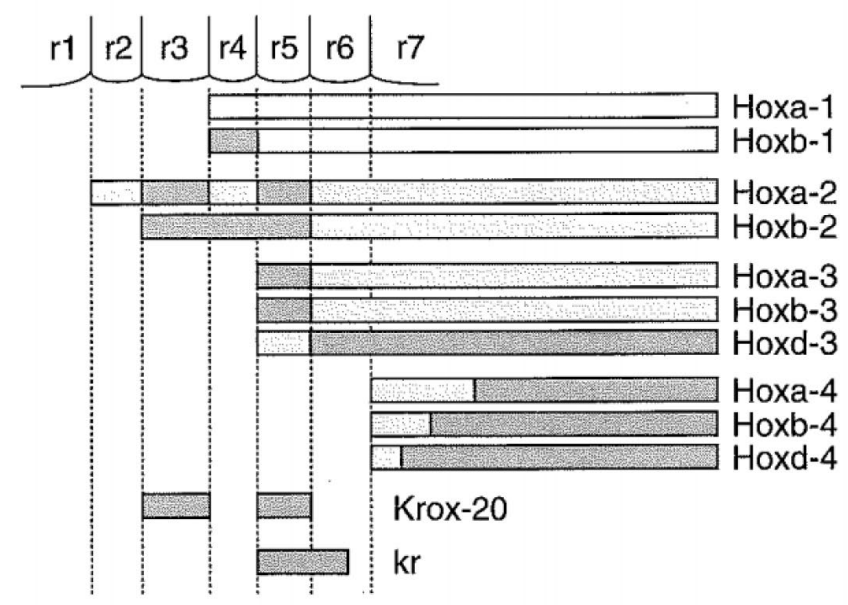

Irving, Flenniken, Alldus, \& Wilkinson, 1996
C E11.5

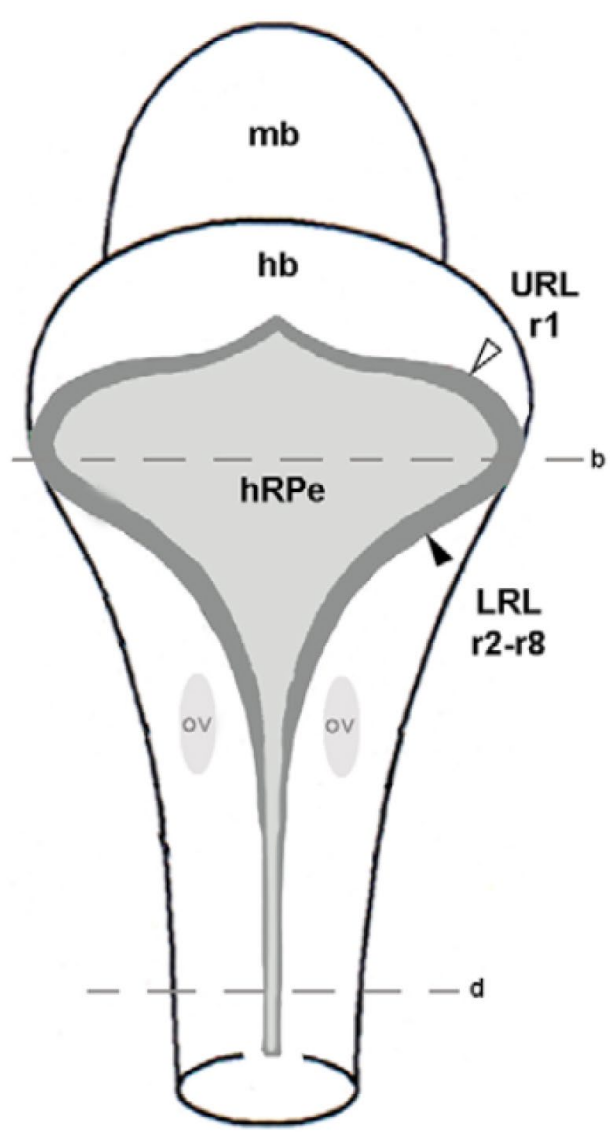

Hunter and Dymecki, 2006

Figure 2: Rhombomeres of the Mouse Hindbrain. A. The mouse hindbrain can be subdivided into seven rhombomeres ( $r 1-7)$, both through morphology and protein expression (b1-3, brachial arches; ov, otic vesicle). B. Multiple proteins, including the homeobox-containing (Hox) proteins, early growth response protein 2 (EGR2 or Krox-20), and V-maf musculoaponeurotic fibroscarcoma oncogene homolog B (MAFB or Kreisler (kr)), are expressed differentially within the developing mouse rhombomeres. Shading indicates relative levels of expression for each protein, with unshaded boxes representing expression prior to the identification of rhombomeres by morphology. C. In the dorsal view of the developing neural tube of the mouse, the rhombic lip can be seen divided into upper (URL) and lower (LRL) segments. The URL is contained within rhombomere 1 $(\mathrm{r} 1)$, while the LRL spans the remaining rhombomeres. Adapted from Hunt et al., 1991; Irving, Flenniken, Alldus \& Wilkinson, 1996; and Hunter and Dymecki, 2006. 
A

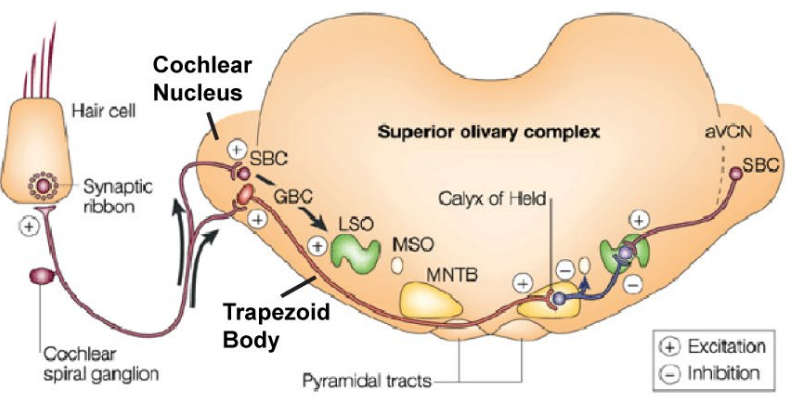

modified from Von Gersdorff and Borst, 2002

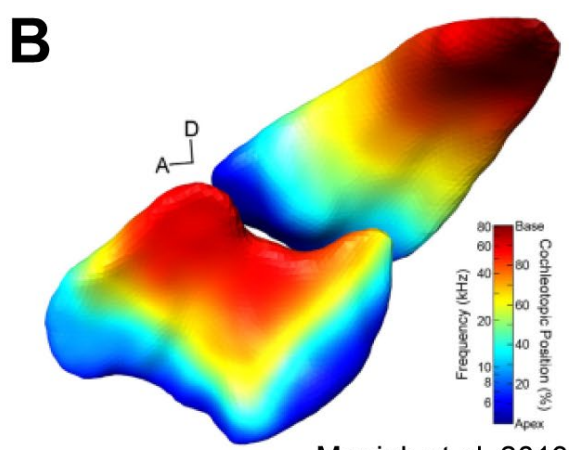

Muniak et al, 2013

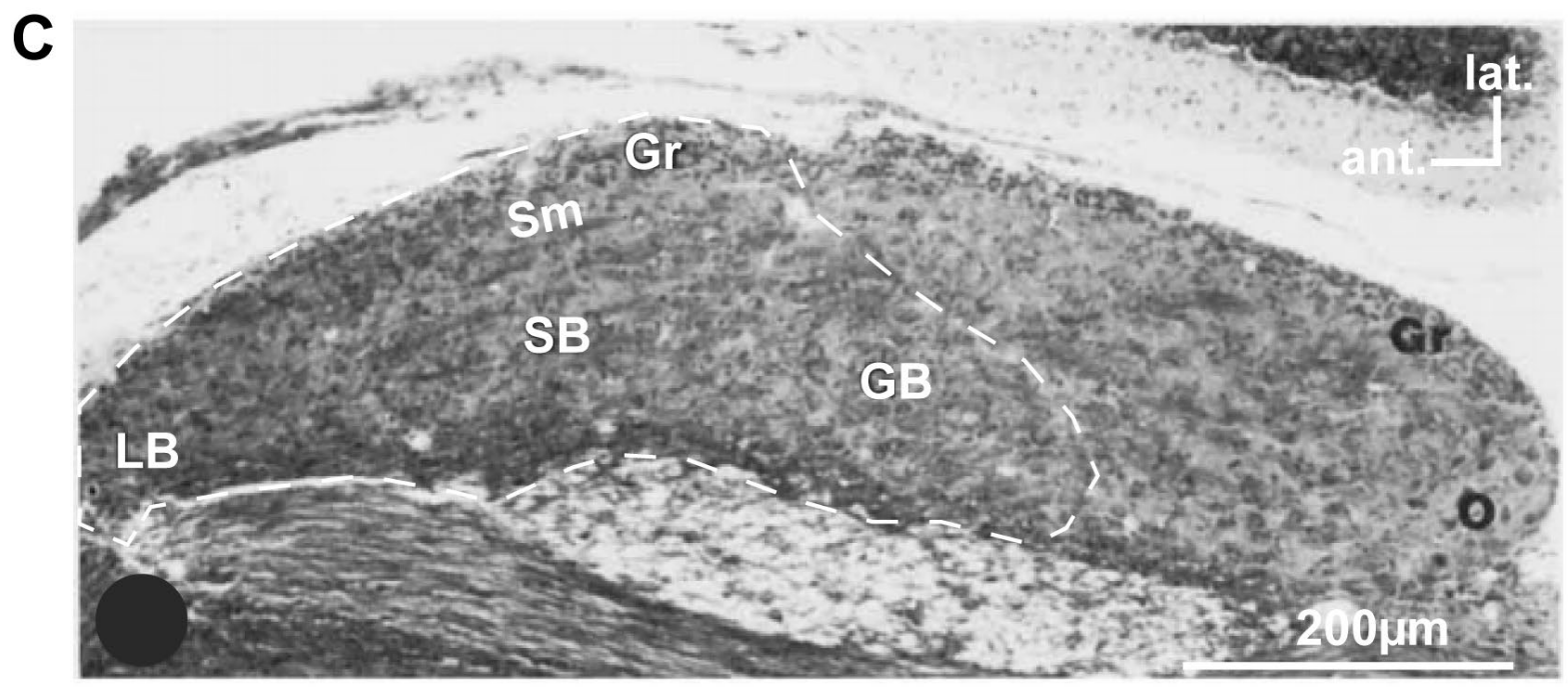

modified from Webster and Trune, 1982

Figure 3: Cochlear Nucleus Anatomy. A. Schematic of major structures of the auditory brainstem sound localization circuit. Transduction of auditory signals from hair cells to the cochlear nucleus is provided by the spiral ganglion neurons. The two major cell types of the anteroventral cochlear nucleus, spherical bushy cells (SBC) and globular bushy cells (GBC) project their axons in a axonal tract called the trapezoid body (or ventral acoustic stria) to multiple nuclei of the superior olivary complex (SOC). In particular, the connection between the GBCs and principal neurons of the contralateral medial nucleus of the trapezoid body (MNTB) - a large axosomatic terminal called the calyx of Held-will be the major focus of this dissertation. B. Innervation of the major subdivisions of the cochlear nucleus by the vestibulocochlear nerve occurs in a pattern that preserves the frequency mapping of the cochlea, a phenomenon called tonotopic mapping. Innervation occurs from low frequency at the most anteroventral areas (blue) progressing to high frequency at the most dorsolateral extents (red). C. Mouse cochlear nucleus (horizontal slice) stained with luxol fast blue-cresyl violet. The anteroventral cochlear nucleus (dotted white line) is comprised of large (LB) and small (SB) spherical bushy cells, globular bushy cells (GB), multipolar cells (throughout), a small cell cap ( $\mathrm{Sm}$ ), and a granule cell layer (Gr). Adapted from Von Gersdorff and Borst, 2002; Muniak et al, 2013; and Webster and Trune, 1982. 

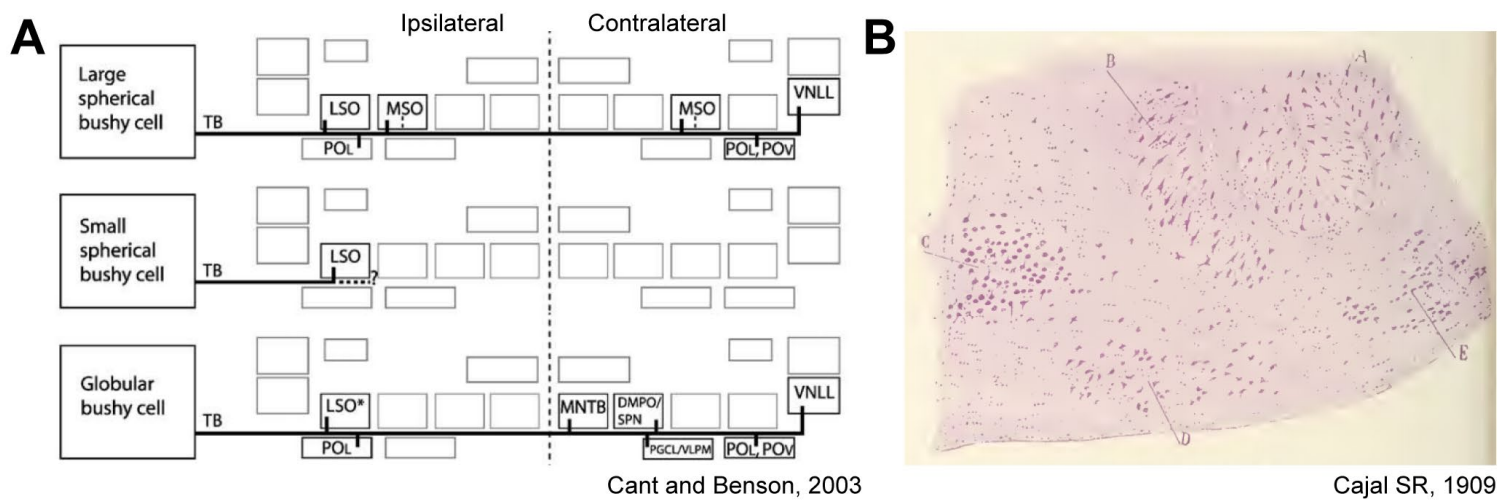

\section{C}

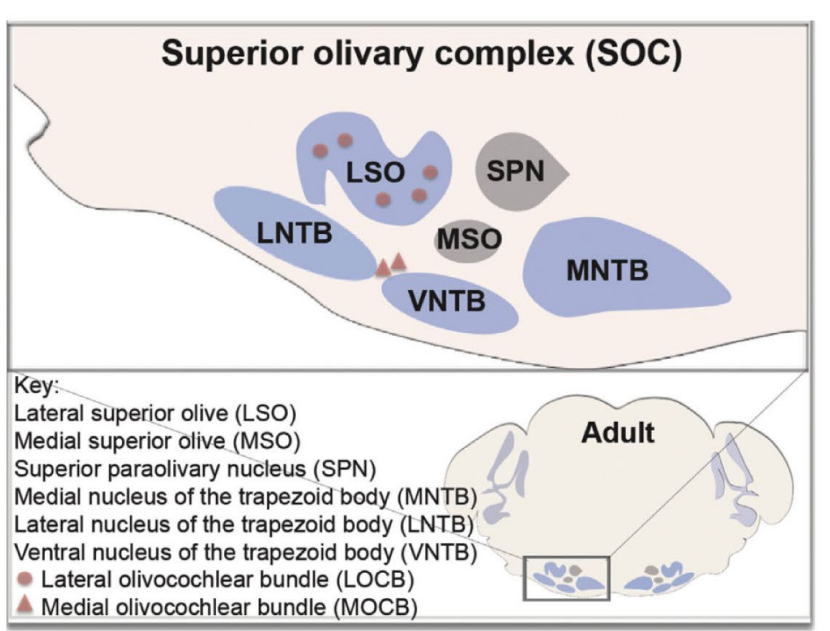

Altieri et al, 2015

A: Superior olive

B: Accessory olive (periolivary nucleus)

C: Nucleus of the Trapezoid Body

$D$ : Internal preolivary ganglion

$\mathrm{E}$ : External preolivary ganglion

TB: Trapezoid body

LSO: Lateral superior olive

MSO: Medial superior olive

VNLL: Ventral nucleus of the lateral limniscus

DMPO: Dorsomedial paraolivary nucleus

SPN: Superior paraolivary nucleus

MNTB: Medial nucleus of the trapezoid body

PGCL: Lateral paragigantocellular nucleus

VLPM: Ventrolateral part of the medullary nucleus

PO: Periolivary nuclei (L: lateral; V: ventral)

Figure 4: Auditory Brainstem Anatomy. A. Connectivity diagram of cochlear nucleus cell output to auditory brainstem nuclei through the trapezoid body. B. Initial characterization of auditory brainstem nuclei using Nissl stain. Five separate nuclei are identified. C. Current accepted subdivision of superior olivary complex (SOC) in the auditory brainstem. Adapted from Cant and Benson, 2003; Cajal SR, 1909; and Altieri et al, 2015. 
A

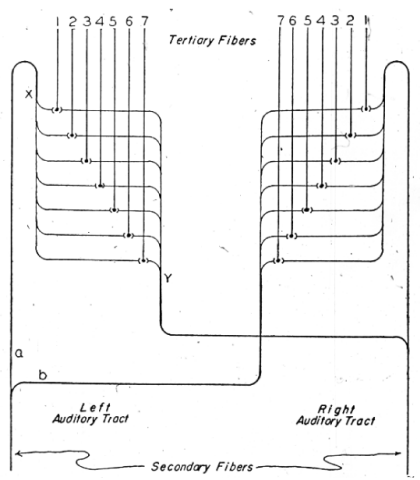

Jeffress LA, 1948

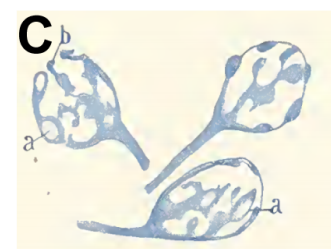

Cajal R, 1909

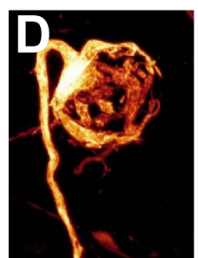

Kuner T, 2004
B

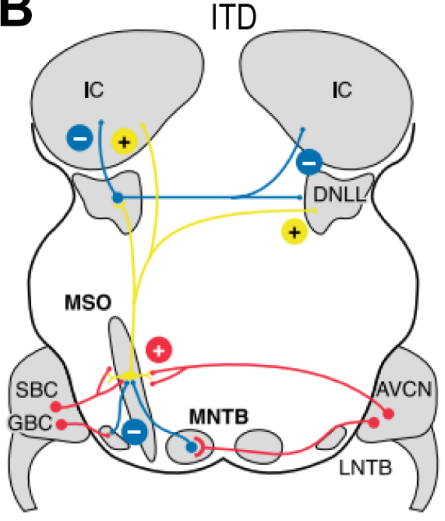

AVCN: Anterio-ventral cochlear nucleus

DNLL: Dorsal nucleus of the lateral lemniscus

GBC: Globular bushy cell

IC: Inferior colliculus

MNTB: Medial nucleus of the trapezoid body

MSO: Medial superior olive

SBC: Spherical bushy cell

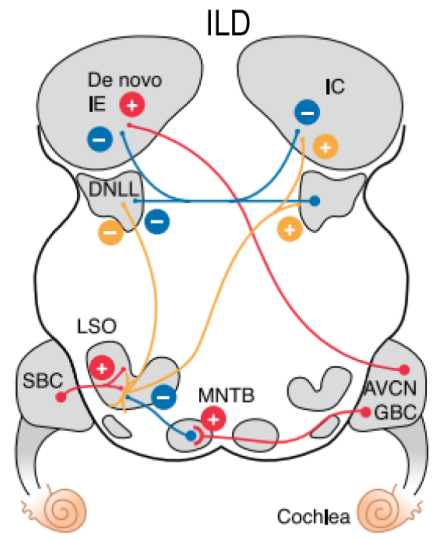

AVCN: Anterio-ventral cochlear nucleus

DNLL: Dorsal nucleus of the lateral lemniscus GBC: Globular bushy cell

IC: Inferior colliculus

LSO: Lateral superior olive

MNTB: Medial nucleus of the trapezoid body

SBC: Spherical bushy cell

Grothe, Pecka, and McAlpine, 2010

Figure 5: Connectivity of Auditory Nuclei for Binaural Sound Localization. A. Delay line schematic as envisioned by Jefress to describe the coincidence detection of sound by two ears. B. Circuit diagram of the auditory brainstem describing the pathways for computation of interaural time differences (ITD) and interaural level differences (ILD). C. Adult calyces of Held as depicted by Ramón y Cajal from terminals stained using methylene blue. D. Adult calyx of Held imaged by Tom Kuner after in vivo expression of virally transfected green fluorescent protein. Adapted from Jefress LA, 1948; Grothe, Pecka, and McAlpine, 2010; Cajal SR, 1909; and Kuner T, 2004 (https://www.uni-heidelberg.de/izn/researchgroups/kuner-t/currentresearch.html). 

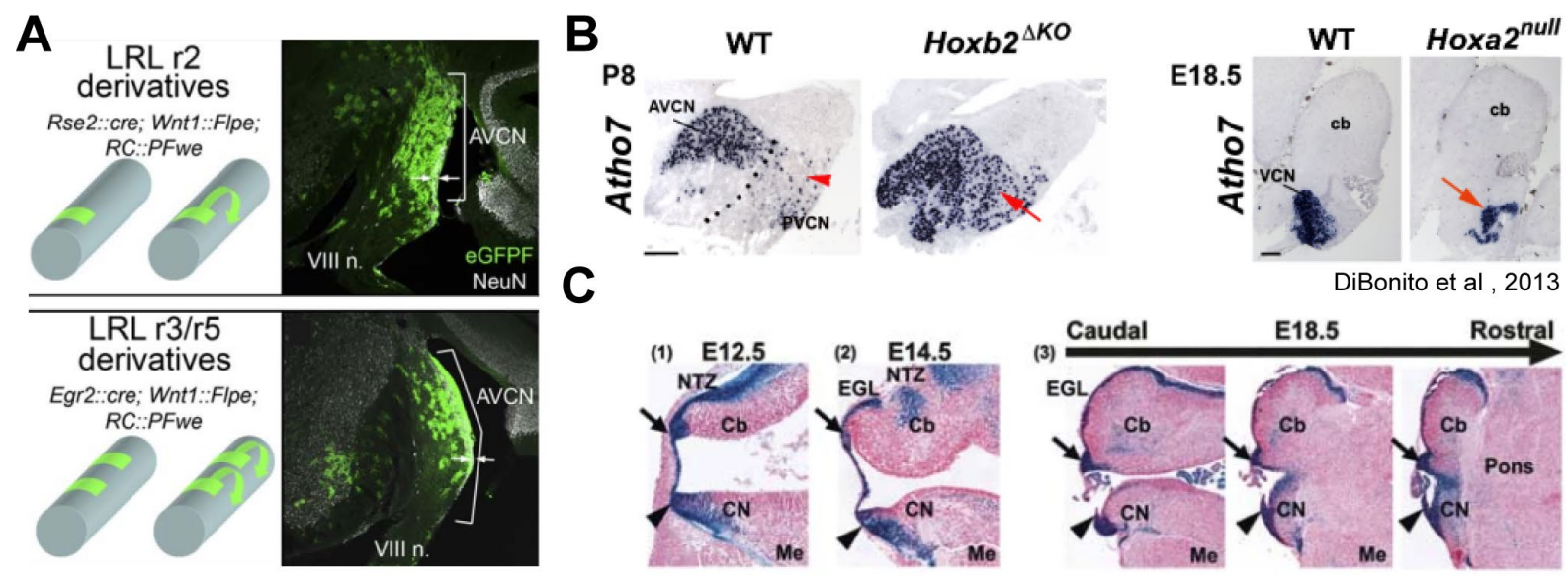

Farago, Awatramani, and Dymecki, 2003

Figure 6: Embryonic Origins of the Anteroventral Cochlear Nucleus (AVCN). A. AVCN neurons are derived from the lower rhombic lip of rhombomeres 2 and 3. Enhanced GFP (green) is expressed in these cells only following successful recombination from both Wnt1::Flpe and either Rse2::cre (r2-specific) or Egr2::cre (r3/5specific). Cells were also stained with NeuN, a neuron-specific label. Entrance of VIIIth nerve labeled for reference. B. Coronal sections of cochlear nucleus from P8 (left) or E18.5 (right) mice stained for Atoh7 (Math5), a protein specific to spherical and globular bushy cells. A very low level of expression of Atoh7 is visible in the posteroventral cochlear nucleus (PVCN, red arrowhead) in wild type (WT) animals, while Atoh7 is highly expressed in the PVCN in Hoxb2 ${ }^{\triangle K O}$ mice (left red arrow) suggesting these cells have adopted an AVCN phenotype. Conversely, loss of Hoxa2 (Hoxa2 ${ }^{\text {null }}$ ) leads to a dramatic reduction in the size of the AVCN (right red arrow). C. Coronal sections through mouse embryos show $\beta$-gal expression under control of Math1 in the cochlear extramural stream (CES) from the rostral (arrow) and caudal (arrowhead) rhombic lips. Cells migrating through this stream form both the cerebellum $(\mathrm{Cb})$ and the cochlear nucleus (CN). Medulla (Me) and pons labeled for reference. Adapted from Farago, Awatramani, and Dymecki, 2003; DiBonito et al, 2013; Wang, Rose, and Zoghbi, 2005. 

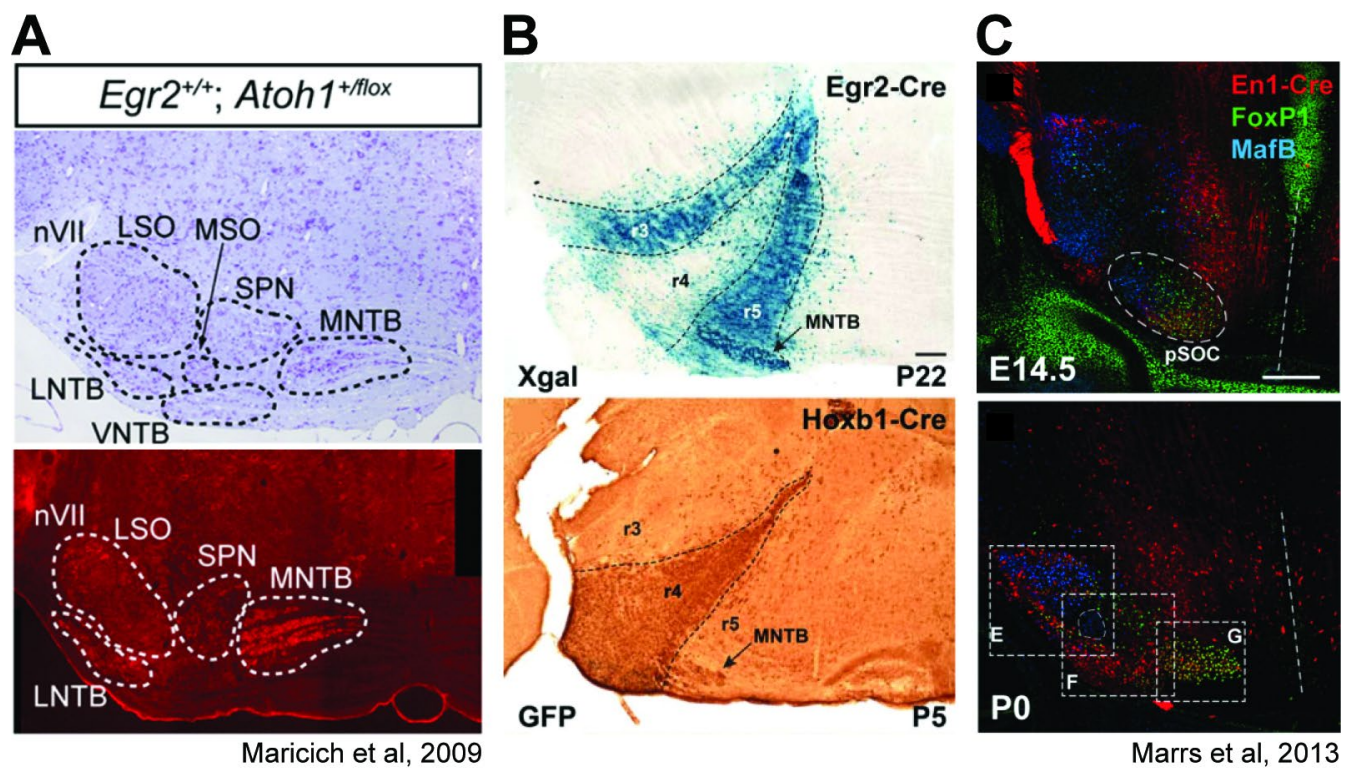

Figure 7: Embryonic Origins of the Medial Nucleus of the Trapezoid Body (MNTB). A. Superior olivary complex (SOC) neurons in 7-week-old mice stained with cresyl violet (top) and Cat-301, an extrasynaptic cell surface marker (bottom). Egr2 (Krox20), expressed in rhombomeres 3 and 5, is expressed in all SOC nuclei: lateral and medial superior olives (LSO, MSO); lateral, ventral, and medial nuclei of the trapezoid body (LNTB, VNTB, MNTB); and superior paraolivary nucleus (SPN). Facial nerve (nVII) labeled for reference. B. Xgal staining of Egr2-positive rhombomeres 3 and 5 ( $r 3$ and $r 5$, blue, top) and GFP staining of Hoxb1positive rhombomere 4 (r4, dark brown, bottom) show the rhombomeric origins of the MNTB. C. Presumptive $\mathrm{SOC}$ (pSOC) nuclei can be identified as early as E14.5 in the mouse using a combination of three novel markers (dotted white oval). By P0, these nuclei have separated (LSO and LNTB, box E; SPN, VNTB, and MSO, box F; and MNTB, box G). MNTB cells are En1-positive (red) and FoxP1-positive (green). Adapted from Maricich et al, 2009; Marrs et al, 2013. 


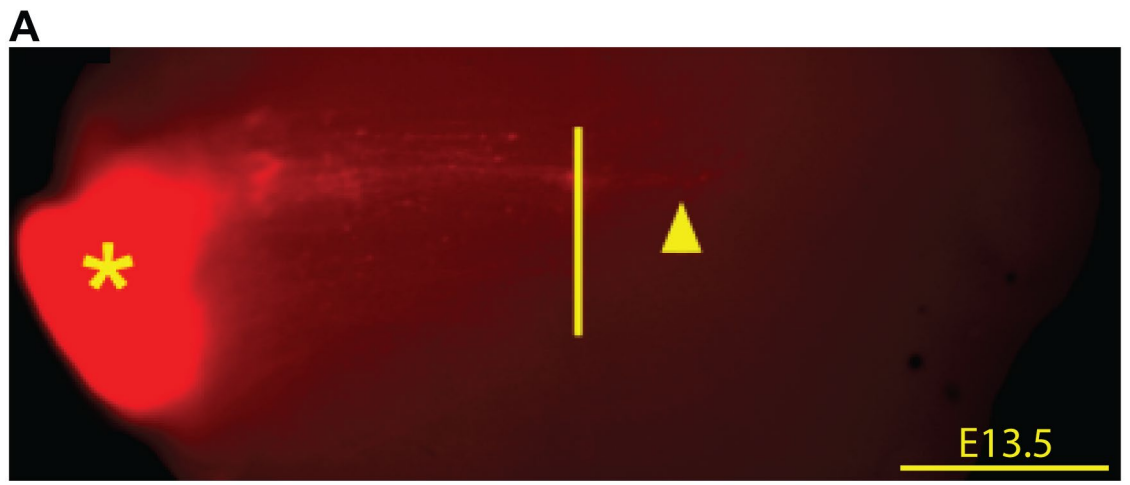

B

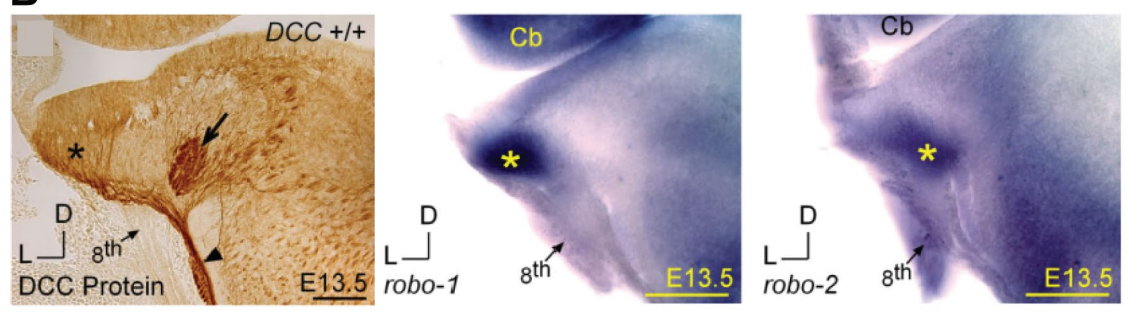

C
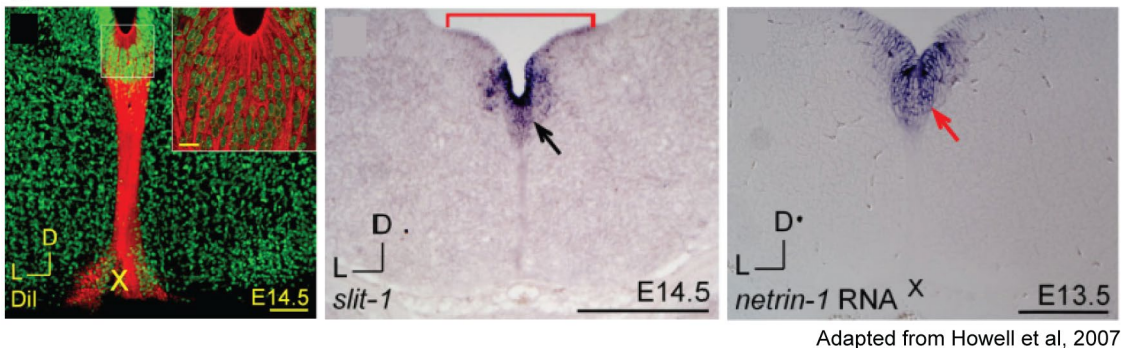

Figure 8: VCN Axon Guidance is Mediated by DCC-Netrin and Slit-Robo Binding. A. Dil/DiL injections into the VCN (asterisk) at E13.5 show axons (red lines) crossing the midline (yellow vertical line) and decussating in an area analogous to that of the adult MNTB (yellow arrowhead). B. DCC protein (left, dark brown) is expressed in axons (arrowhead) leaving the cochlear nucleus (asterisk) and within the cerebellar peduncle (arrow) at E13.5. Robo-1 and robo-2 mRNAs (middle and right, respectively) are also expressed in the cochlear nucleus at this age. C. Neurons that reside in the midline (green, SYTOX green stain, left and inset) project their axons along the midline (red, DiD stain). These cells appear to express both slit-1 (middle arrow) and netrin-1 (right red arrow) mRNA. Dorsal and lateral directions $(\mathrm{D}, \mathrm{L})$ are indicated, and cerebellum $(\mathrm{Cb})$ and $8^{\text {th }}$ nerve are labeled for reference. $X$ label indicates ventral surface of the brainstem. Scale bars

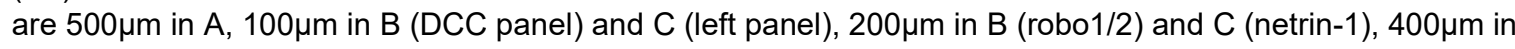

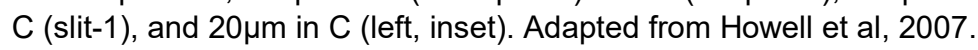



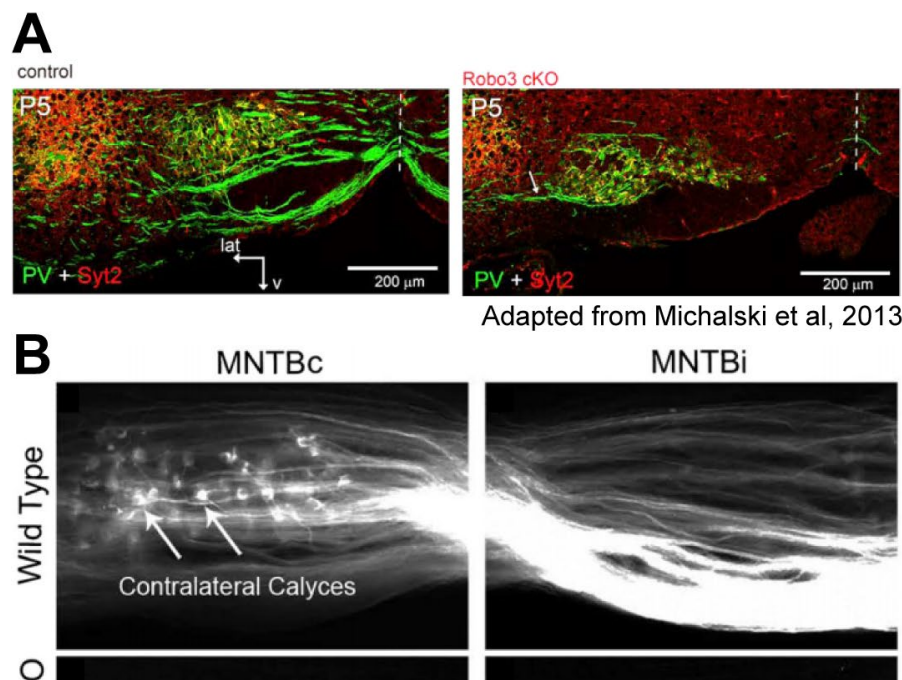

Adapted from Michalski et al, 2013

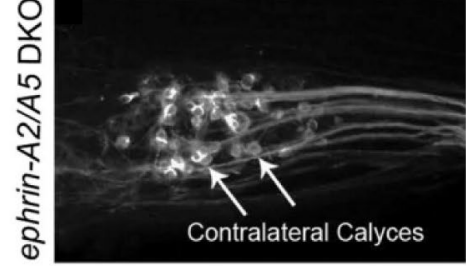

MNTBi
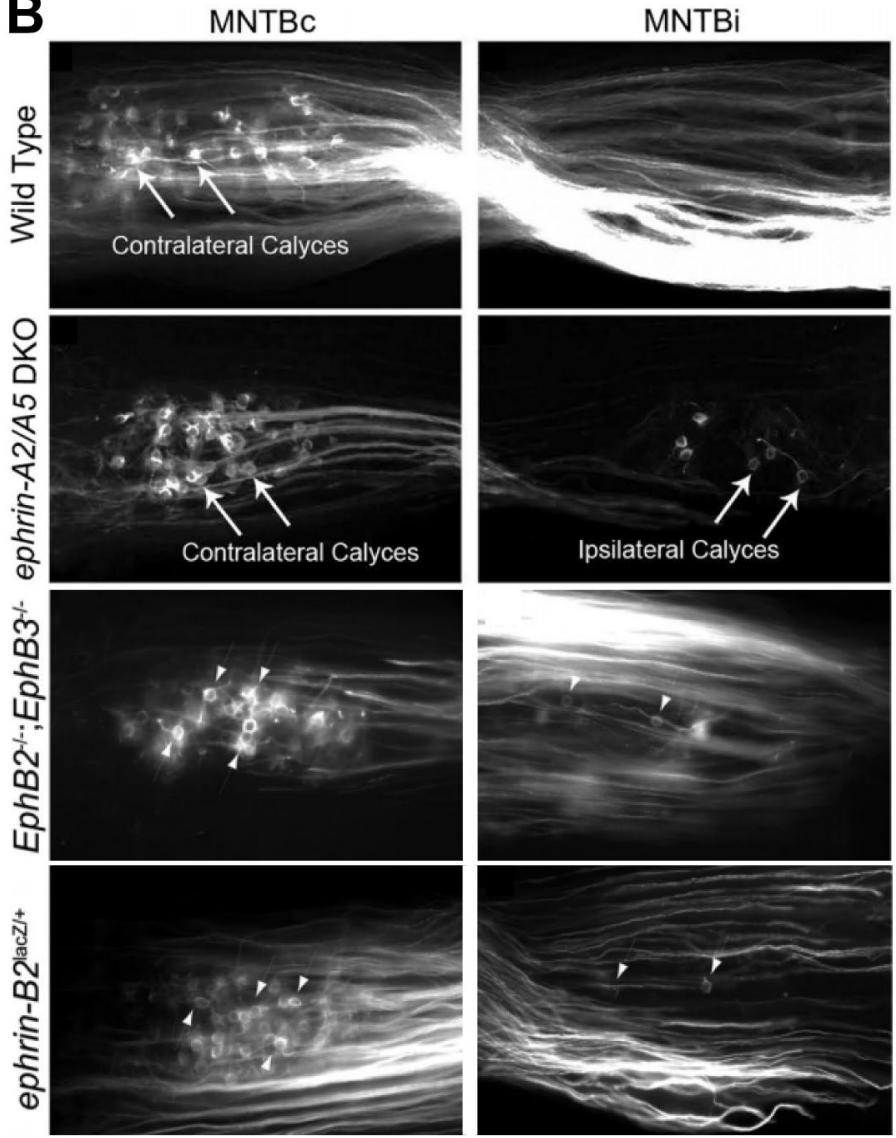

Adapted from Abdul latif et al, 2015, and Hsieh et al, 2010

Figure 9: Eph-ephrin Binding Inhibits Ipsilateral Calyx Formation. A. In wild type animals (left), GBC axons (green) cross the midline (dotted line) and form calyces (yellow) in the contralateral MNTB. When midline crossing is disrupted by knockout of Robo3 (right), GBC axons (arrow) instead form calyces in the ipsilateral MNTB. PV, parvalbumin; Syt2, synaptotagmin-2. Lateral (lat) and ventral (v) directions labeled for reference. B. Although formation of the calyx of Held in the contralateral MNTB (MNTBC) is not disrupted in knockout models of Eph and ephrin, aberrant calyces form in the ipsilateral MNTB (MNTBi). Importantly, no ipsilateral calyces form in EphB3 knockout alone (data not shown). Axons/calyces labeled by placement of NeuroVue Red dye unilaterally in the VCN. Adapted from Michalski et al, 2013; Abdul latif et al, 2015; and Hsieh et al, 2010. 


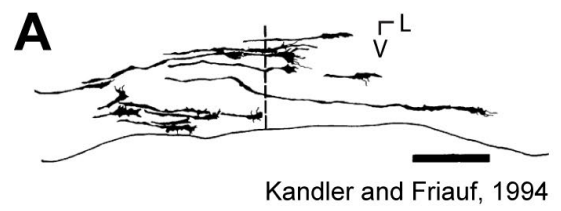

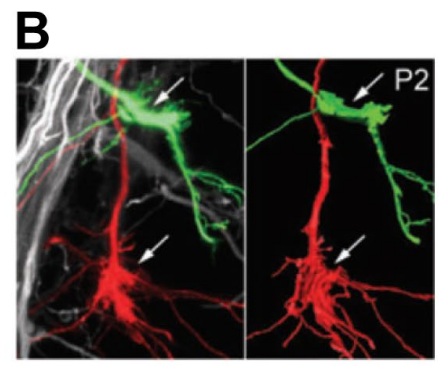

C
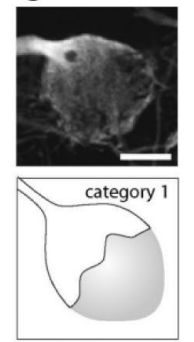

$10 \mu$

Morest K, 1968
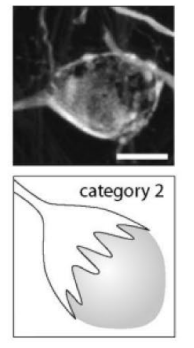

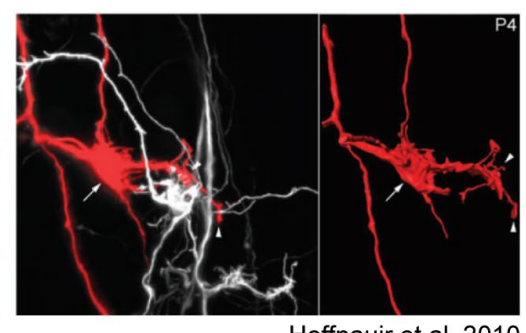

Hoffpauir et al, 2010
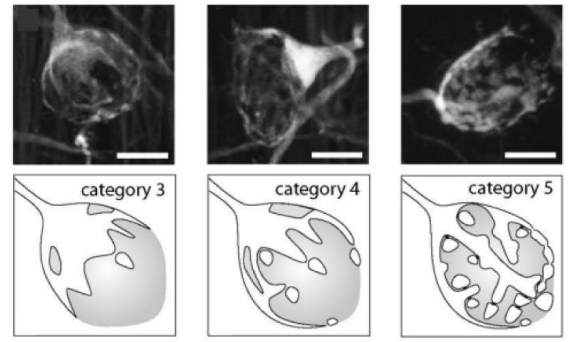

Ford et al, 2009

Figure 10: Morphological Development of the Calyx of Held. A. As early as E15, GBC axons in the rat (top) can be seen crossing the midline (dotted line) possessing elongated growth cones with filopodial- or lamellipodial-like endings (scale bar: $50 \mu \mathrm{m}$; L - lateral, V - ventral). In the cat, protocalyces can be seen shortly after birth (1 in bottom panel, P0-P2), while young unfenestrated calyces are present at P3 $(2,3,4)$ and P7 (5). Both thin sinuous processes (arrow) and thicker processes resembling growth cones (arrowhead) can be seen at these ages. B. Protocalyces in the mouse can be identified as early as P2 (arrows, left panel), and by P4 young calyces have formed (arrow, right panel). Like the calyx in A4, a thick calyceal appendage with the appearance of a growth cone can be seen projecting from the calyx (arrowheads). Calyces in the left panels were labeled with fluorescent dextrans electroporated into the VCN axon between the midline and medial edge of the MNTB, while those in the right panels represent 3D reconstructions of the calyces in the left panels. C. Following the onset of hearing, young calyces undergo a morphological reorganization called fenestration. As defined by Ford et al, the stages of fenestration are completely closed (category 1), early fenestrated (category 2), intermediate fenestrated (category 3), late fenestrated (category 4), and completely fenestrated (category 5). Adapted from Kandler and Friauf, 1994; Morest K, 1968; Hoffpauir et al, 2010; and Ford et al, 2009. 

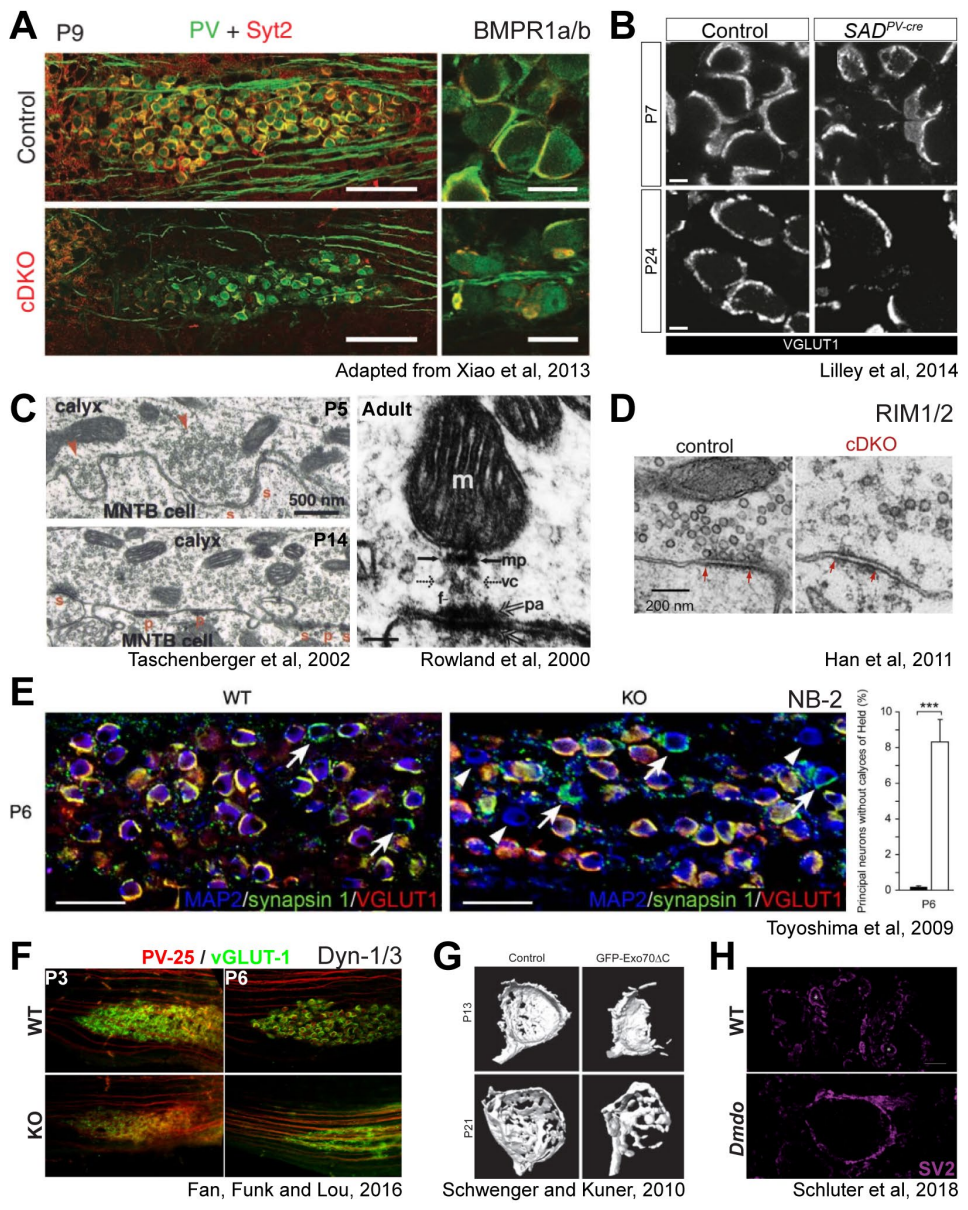

Figure 11: Protein Pathways Involved in Calyx Development. A. In P9 wild type mice (top), young calyces (yellow) can be seen surrounding all MNTB principal cells (green). These calyces have a solid, cupshaped morphology (top right). However, conditional knockout of BMP receptors $1 \mathrm{a}$ and $1 \mathrm{~b}$ cause both significant loss of calyces (bottom left) and a more immature, spoon-shaped morphology (bottom right).

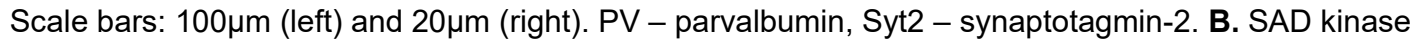
appears to have little influence on early morphological formation of the calyx of Held, as is evident from the similarity of VGLUT1 staining between control (top left) and SAD conditional knockout mice (top right) at P7. By P24, however, SAD knockouts (bottom right) occupy significantly less somatic territory ( $33 \%$ ) than agematched controls (bottom left). Scale bar: $5 \mu \mathrm{m}$. C. Synapses (s) and vesicle clusters (red arrowheads) in the P5 rat (top left) are larger than at P14 (bottom left). The structure of the synapse-associated MAC (right), as detailed by Rowland et al, is composed of a mitochondrion $(\mathrm{m})$, mitochondrial plaque $(\mathrm{mp})$, vesicle chain $(\mathrm{vc})$, filaments (f), and punctum adherens ( $\mathrm{p}$ in left images, pa in right image). Scale bars: $500 \mathrm{~nm}$ (left) and 100nm (right). D. RIM1/2 double knockouts (right) show a significant distance from the synapse (red arrows) to vesicles compared to controls (left). E. NB-2 (contactin 5) knockout mice show significantly higher percentages of principal cells without calyces (arrowhead, right panel) compared to wild type (left). Scale bar: $50 \mu \mathrm{m}$. MAP2 - microtubule associated protein 2, VGLUT1 - vesicular glutamate transporter 1 . F. While dynamin-1/3 knockout mice (bottom) show no phenotypic difference compared to wild type (top) prior to significant growth of the calyx at P3 (left panels), significant morphological differences are evident by P6 (right panels). PV-25 - parvalbumin. G. 3D reconstructions from confocal image stacks show significant volumetric differences in the calyx between $\mathrm{P} 13$ control (top left) and Exo70 dominant negative-expressing (top right) rats. Although calyx volume has recovered by P21, differences in morphology are still apparent (bottom). H. Adult mice with a miR-96 mutation (Dmdo, bottom) possess an immature calyceal morphology in comparison to their compartmentalized wild type counterparts (top). SV2 - synaptic vesicle protein 2. Adapted from Xiao et al, 2013; Lilley et al, 2014; Taschenberger et al, 2002; Rowland et al, 2000; Han et al, 2011; Toyoshima et al, 2009; Fan, Funk, and Lou, 2016; Schwenger and Kuner, 2010; Schluter et al, 2018. 


\section{A}
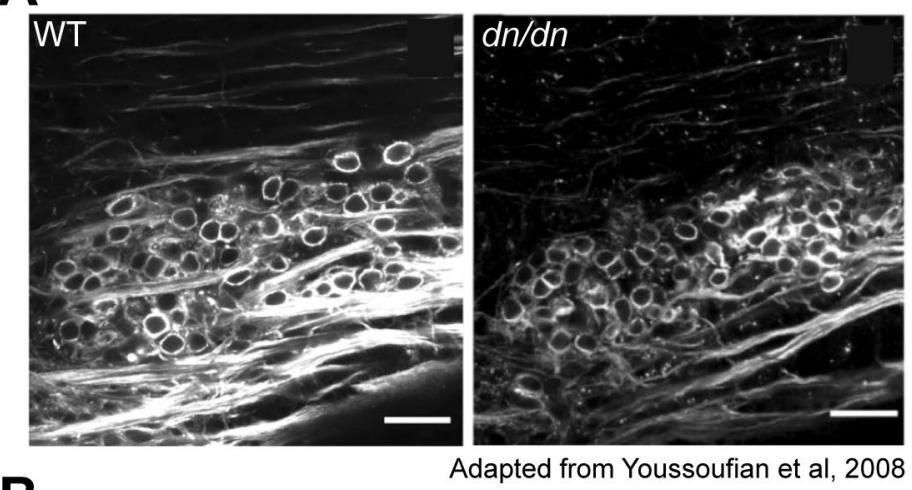

B
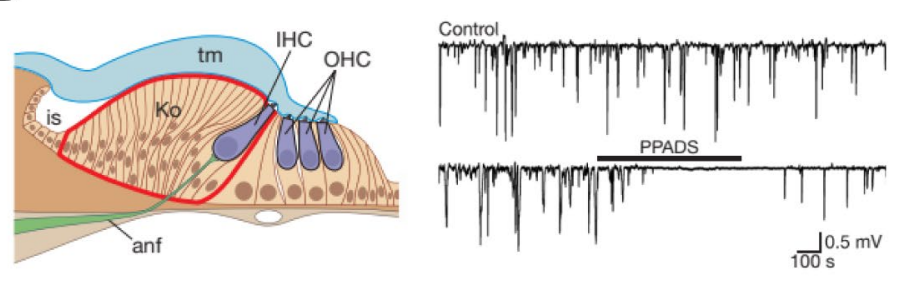

C

a

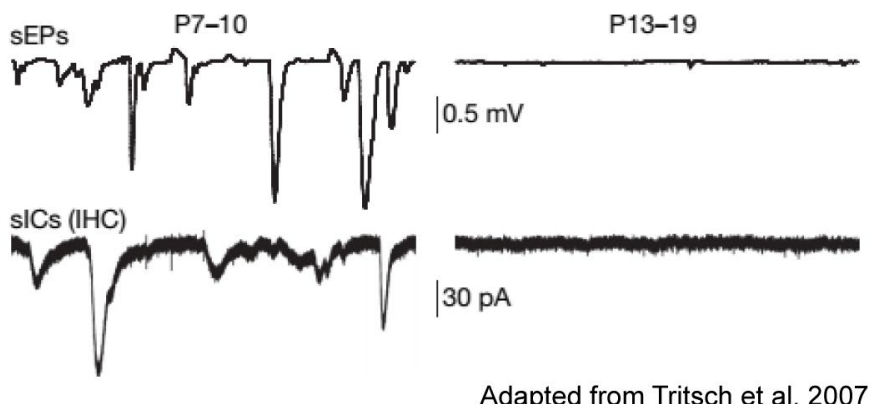

Figure 12: Spontaneous Activity in the Pre-hearing Auditory System. A. No morphological difference in

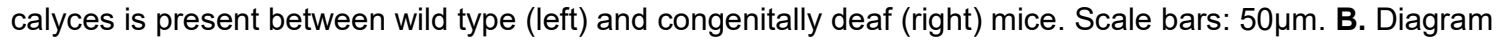
of organ of Corti in the P7 rat (left) including the tympanic membrane (tm), inner sulcus (is), inner and outer hair cells (IHC, OHC), Kölliker's organ (Ko), and auditory nerve fiber (anf). Spontaneous activity in Kölliker's organ (top trace) is mediated by ATP, as evidenced by abolishment of signal upon addition of a P2 purinergic receptor antagonist, PPADS (lower trace). C. Spontaneous extracellular potentials (sEPs) at Kölliker's organ and spontaneous inward currents (sICs) at inner hair cells are prominent prior to hearing onset (P7-10, left) and abolished after opening of the ear canal (P13-19, right). Adapted from Youssoufian et al, 2008; and Tritsch et al, 2007. 


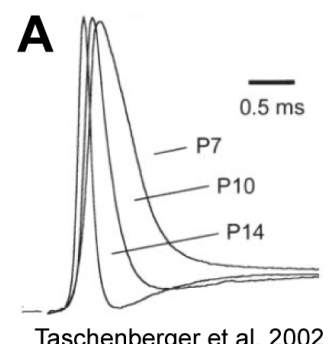

Taschenberger et al, 2002

D

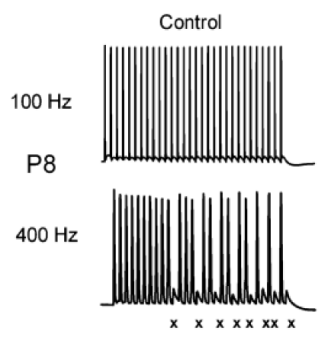

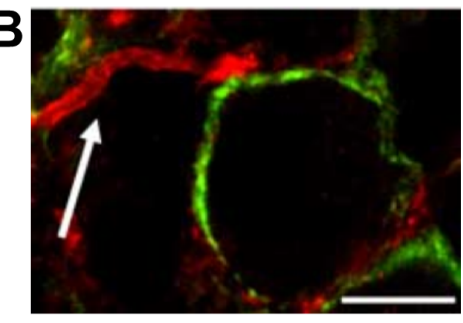

Leão et al, 2005

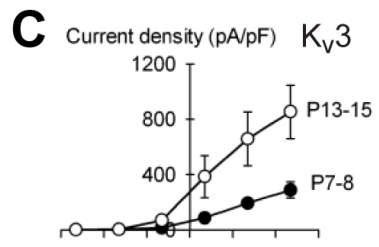

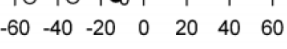
Voltage $(\mathrm{mV})$

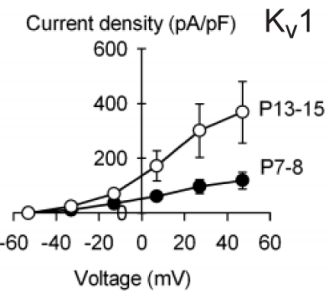

Nakamura and Takahashi, 2007

$\mathbf{E}$

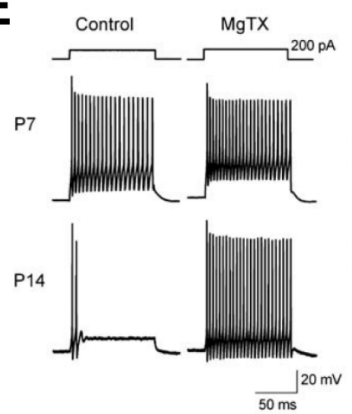

dapted from Nakamura and Takahashi, 2007

Figure 13: Changes in Calyceal Na+ and K+ Channels Sharpen the Presynaptic Action Potential. A. Developmental sharpening of the calyceal action potential from P7-14 in the rat. B. Sodium channels (red, anti-pan-NA antibody) are excluded from the calyx (green, anti-SV2 (synaptic vesicle protein 2) antibody) and are instead found on the extended heminode of the GBC axon (arrow). Scale bar: 10 $\mu \mathrm{m}$. C. Current density of both $\mathrm{K}_{\mathrm{v}} 3$ (left) and $\mathrm{K}_{\mathrm{v}} 1$ (right) channels increase during the second postnatal week. D. Afferent fiber stimulation at $100 \mathrm{~Hz}$ (top row) and $400 \mathrm{~Hz}$ (bottom row) had differential effects based on age and $\mathrm{K}^{+}$ channel blockage. At all ages, calyceal action potentials could follow a $100 \mathrm{~Hz}$ stimulation, while younger ages (P8) failed to maintain firing with $400 \mathrm{~Hz}$ ( $\mathrm{X}$ on bottom of trace). Although control animals at P14 (bottom right) showed no failure to maintain fidelity at $400 \mathrm{~Hz}$, inhibition of $\mathrm{K}^{+}$channels with IbTX + TEA (blocking $\mathrm{K}_{\mathrm{v}} 3$

channels) caused significant failures akin to those at P8. E. A single, 100ms depolarizing current (square waveform at top) evoked tonic firing at P7 (top left trace) and phasic firing at P14 (bottom left trace). In the presence of margatoxin-a scorpion venom derivative that blocks $\mathrm{K}_{\mathrm{v}} 1.3$ channels - the $\mathrm{P} 14$ response (bottom right trace) returned to a tonic phenotype, while P7 was unaffected (bottom left trace). Adapted from Taschenberger et al, 2002; Leão et al, 2005; and Nakamura and Takahashi, 2007. 
A

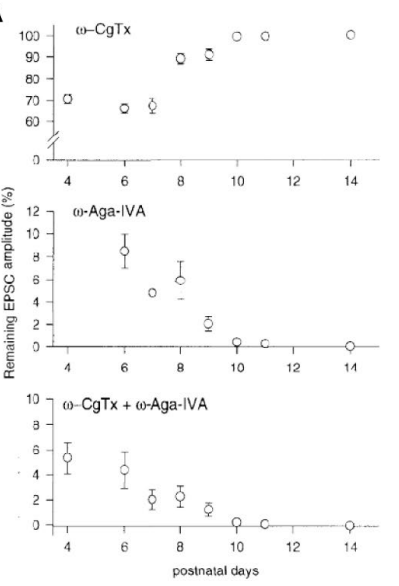

Iwasaki and Takahashi, 1998
B

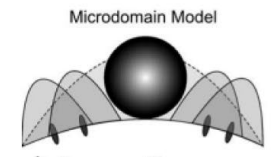

I cä Channel $O$ veside

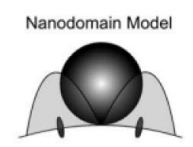

C
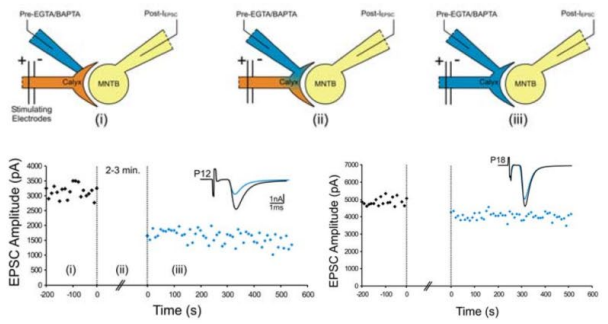

Fedchyshyn and Wang, 2005

D

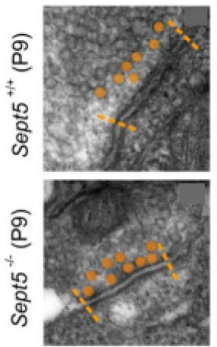

E

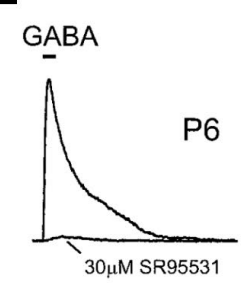

6

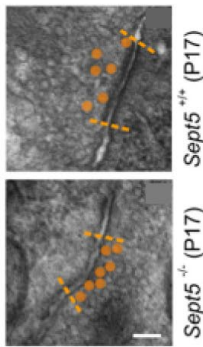

Glycine

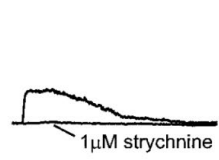

GABA

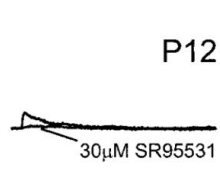

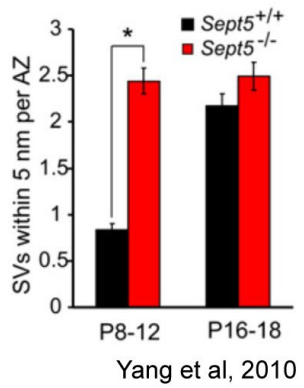

Glycine

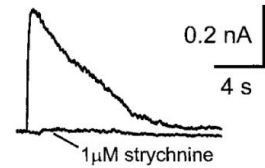

Turecek and Trussell, 2002

Figure 14: Changes in Voltage Gated Calcium Channels (VGCCs) during Calyx Development. A. Measurement of EPSCs in the principal cell of the rat in the presence of two subtype-specific VGCC blockers, $\omega$-conotoxin GVIA ( $\omega$-CgTx, N-type specific) and $\omega$-agatoxin-IV A ( $\omega$-Aga-IV A, P/Q-type specific). While contributions from P/Q-, N-, and R-type VGCCs are evident early in development, only P/Q-type VGCCs are active later. B. Schematic representation of the shift from micro- to nanodomain models in the developing calyx. Early in development, the distance between VGCCs and vesicles necessitates the integration of multiple open VGCCs to elicit release (left). Greater coupling between vesicles and VGCCs later in development decreases the number of VGCCs necessary to cause vesicle release (right). C. VGCCvesicle coupling is experimentally probed using calcium buffers. After calyx patch and EPSC control recordings (i), EGTA and BAPTA (calcium chelators) are infused for 2-3 minutes (ii) and EPSCs are recorded again (iii). A significant decrease in the influence of EGTA/BAPTA is seen at P18 (blue, bottom right) compared to P12 (blue, bottom left), suggesting decreased VGCC-vesicle distance. D. Septin-5 (Sept5) creates a filamentous network that keeps vesicles distanced from the presynapse in P9 mice (top left panels), a phenomenon that is weakened in more mature animals (P17, top middle panels). Although loss of Sept5 causes no significant difference in vesicle distance in P17 animals (bottom middle panels), vesicles at $\mathrm{P} 9$ are no longer distance restricted (bottom left panels, summary in right panel). Scale bar: $100 \mathrm{~nm}$. E.

GABA and glycine both elicit depolarizing currents at the calyx early in development, with a developmental shift from GABA-dominated at P6 (left two traces) to glycine-mediated (right two traces). Adapted from Iwasaki and Takahashi, 1998; Fedchyshyn and Wang, 2005; Yang et al 2010; Turecek and Trussell, 2002. 

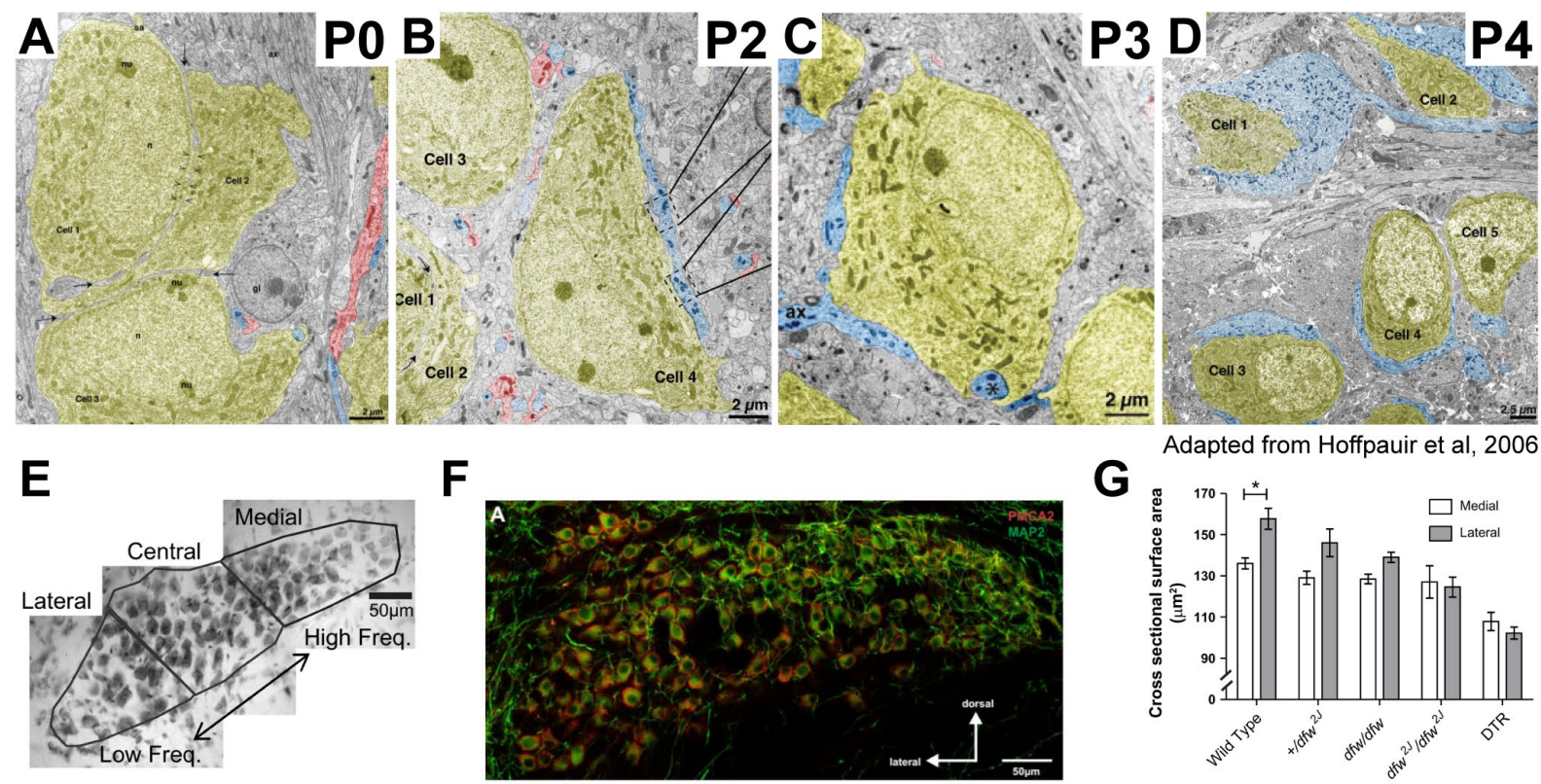

Adapted from Hoffpauir et al, 2006

Adapted from Weatherstone et al, 2016

Figure 15: Morphological Changes in the Developing Principal Cell of the MNTB. A. Principal cell (PC) bodies (gold) are in close apposition at P0, with lengths of cell-cell contact (open arrowheads between cell 1 and 2) and thin spaces between cells filled by glial processes (arrows). One small input (blue) is somatically associated, while the rest are associated with PC dendrites (red). ax - axon bundle, gl - glial cell, n nucleus, nu - nucleolus. B. Cells are still in close apposition at P2 (arrows, cells 1 and 2). Although most of the synaptic contacts appear dendritic at this age, a large protocalyceal input (blue) is apparent on cell 4.C. Cells at P3 are more widely spaced, rounder, and somatic inputs are more prevalent. D. While some cells are still closely apposed at P4 (cells 4 and 5), most have moved apart. Cells have also elongated and attained an oval shape. Very large inputs are present on every cell, with some (see inputs to cells 1 and 4 ) having the cup-shaped appearance of a young calyx. E. Nissl stained cells in the MNTB showing a gradient in cell size from larger cells laterally to smaller cells medially. F. PMCA2 (plasma membrane calcium APTase 2 ) is present both pre- and postsynaptically in the MNTB. G. Quantification of PC cross-sectional area in medial and lateral portions of the MNTB. Although lateral PC size is significantly larger than medial cells in wild type, both overall cell size and tonotopic size gradient is incrementally decreased in $+/ d f w^{2 J}$ (one fully functional copy of PMCA2 gene), dfw/dfw (two copies of PMCA2 gene with $60 \%$ less efficiency), $d f w^{2 \mathrm{~J}} / d f w^{2 \mathrm{~J}}$ (genetic deletion of PMCA2), and DTR (deaf mice due to hair cells ablated with diptheria toxin at 4 weeks old). Adapted from Hoffpauir et al 2006; Weatherstone et al, 2016. 

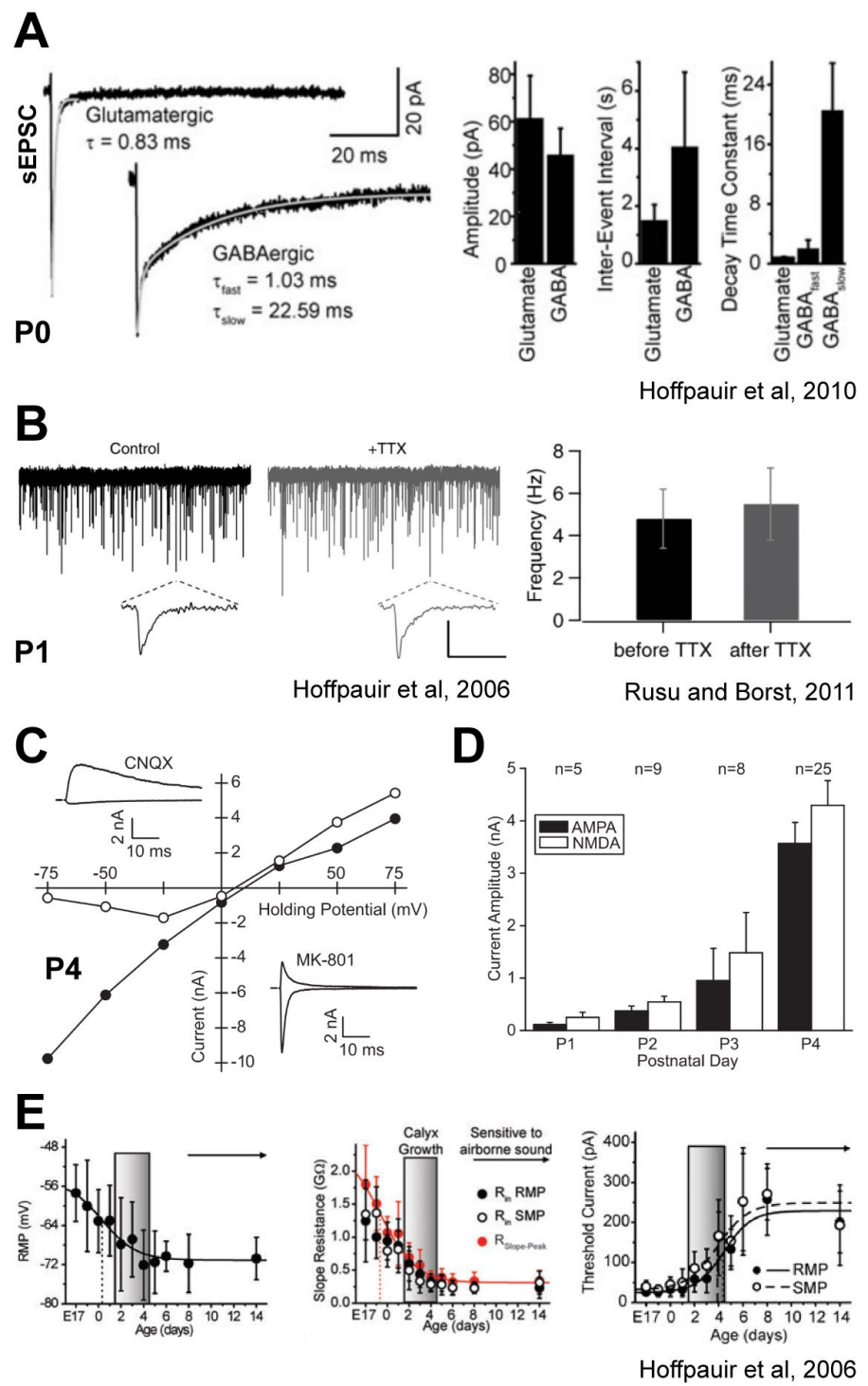

Figure 16: Electrophysiological Changes in the Developing Principal Cell of the MNTB. A.

Spontaneous EPSCs (sEPSC) recorded in the PO PC of the mouse show a fast glutamatergic response (left trace) and slower GABA-ergic response (right trace). The GABA-ergic sEPSCs had a lower amplitude, happened less frequently, and were slower to rectify than their glutamatergic counterparts (graphs on right). B. Spontaneous release from pre-calyx inputs to PCs (left trace) is independent of action potentials as demonstrated by activity in the presence of tetrodotoxin (TTX, right trace), a potent $\mathrm{Na}^{+}$channel blocker. No change in frequency is apparent with or without TTX (graphs on right). C. EPSCs measured for AMPA (•) and NMDA $(\circ)$ receptors at holding potentials from $-75 \mathrm{mV}$ to $+75 \mathrm{mV}$. AMPA receptor currents were blocked by CNQX (top left traces), while NMDA currents were blocked by MK-801 (bottom right traces). Traces were recorded at $-75 \mathrm{mV}$ and $+75 \mathrm{mV}$ holding potential. D. EPSCs evoked by AMPA and NMDA receptors increase from P1-4, with a substantial spike between P3-4. E. As the PC matures, the resting membrane potential (RMP) decreases from $-55.2 \pm 7.4 \mathrm{mV}$ at E17 to near its adult value of $-72.1 \pm 6.4 \mathrm{mV}$ by P4 (left plot). Simultaneously, the membrane resistance decreases as a function of age irrespective of whether the cell is measured at its resting membrane potential $\left(R_{\text {in }} R M P, \bullet\right)$ or held at a standard membrane potential for adults of $-73 \mathrm{mV}\left(R_{\text {in }} S M P, \circ\right)$ (middle plot). While slope resistance was calculated for the whole stimulus range, the maximum resistance for each set of calculated resistances was also plotted as $\mathrm{R}_{\text {Slope-Peak }}(\bullet)$. Consequently, the threshold current necessary to evoke an action potential in the PC increases inversely to these two factors for both RMP (•) and SMP (०). Adapted from Hoffpauir et al, 2006 and 2010; Rusu and Borst, 2011. 
A
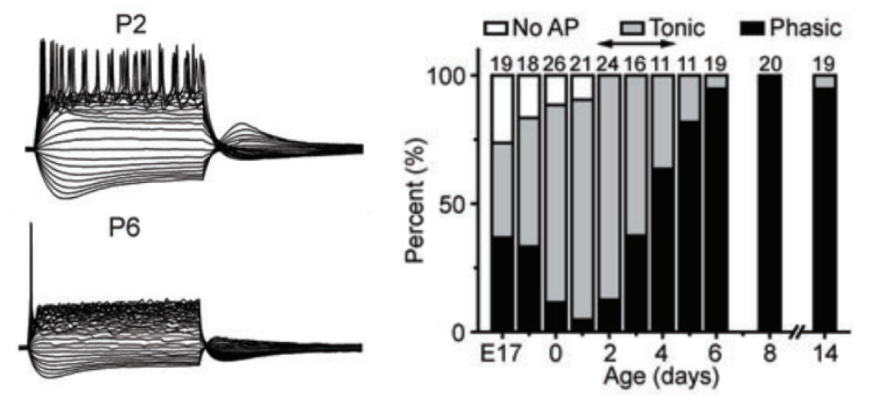

Hoffpauir et al, 2010
B

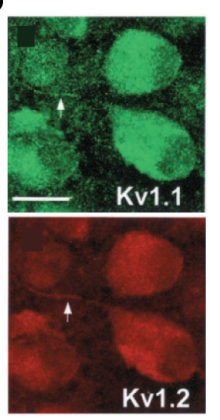

C

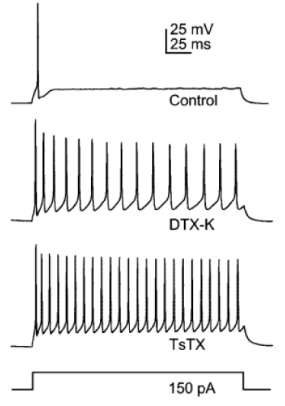

Dodson, Barker, and Forsythe, 2002

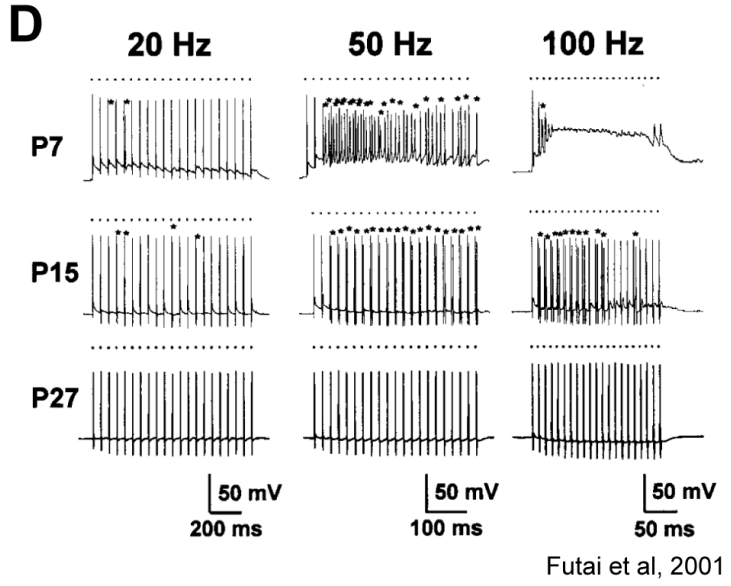

E
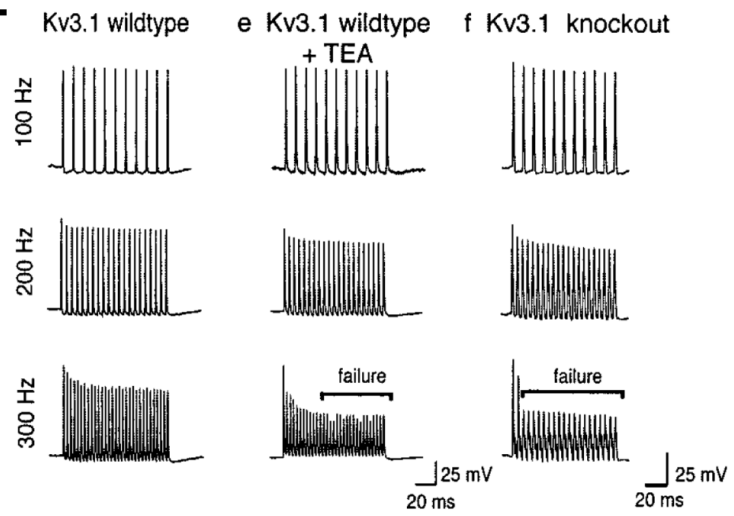

Macica et al, 2003

Figure 17: Maturation of Principal Cell Electrophysiology is Mediated in Part by Potassium Channels. A. Postsynaptic AP firing transitions from a tonic bursting at P2 (top left) to single tonic firing at P6 (bottom left) in response to single depolarizing current injections. Percentages of tonic and phasic firing PCs at each age are plotted on the right. B. Potassium channels Kv1.1 (green) and Kv1.2 (red) are co-expressed in PCs in P8-14 rats, and enriched in the PC axons (arrows). Scale bar: $20 \mu \mathrm{m}$. C. PCs in P8-14 rats that fire phasic APs (top trace) in response to a depolarizing current (bottom trace) can be reverted to tonic firing by inhibiting either Kv1.1 (dendrotoxin, DTX-K; middle top trace) or Kv1.2 (tityustoxin, TsTX; middle bottom trace) channels. D. High-fidelity firing with fewer failures is established in the second week of development and later in the mouse. Stimulation was performed presynaptically using an electrode placed halfway between the midline and the MNTB. At P7, multiple failures are evident at $50 \mathrm{~Hz}$ stimulus (asterisks) while AP firing is abolished at $100 \mathrm{~Hz}$ due to plateau (top traces). By P15, failures have decreased and plateau is no longer present (middle traces). At P27, PCs have achieved high fidelity firing with no failures. E. Kv3.1 channels mediate high-frequency phase locking. Stimulation was performed by direct postsynaptic depolarization with brief current injections of 2nA. While wild type P14 mice (left traces), wild type mice with Kv3.1 channels blocked with TEA (middle traces), and Kv3.1 knockout mice all fire with high fidelity at 100 and $200 \mathrm{~Hz}$, depolarization at $300 \mathrm{~Hz}$ causes significant attenuation in the amplitude of the AP response and failure to fire APs after the initial response in wild type + TEA (bottom middle) and Kv3.1 knockouts (bottom right). Adapted from Hoffpauir et al, 2010; Dodson, Barker, and Forsythe, 2002; Futai et al, 2001; and Macica et al, 2003. 

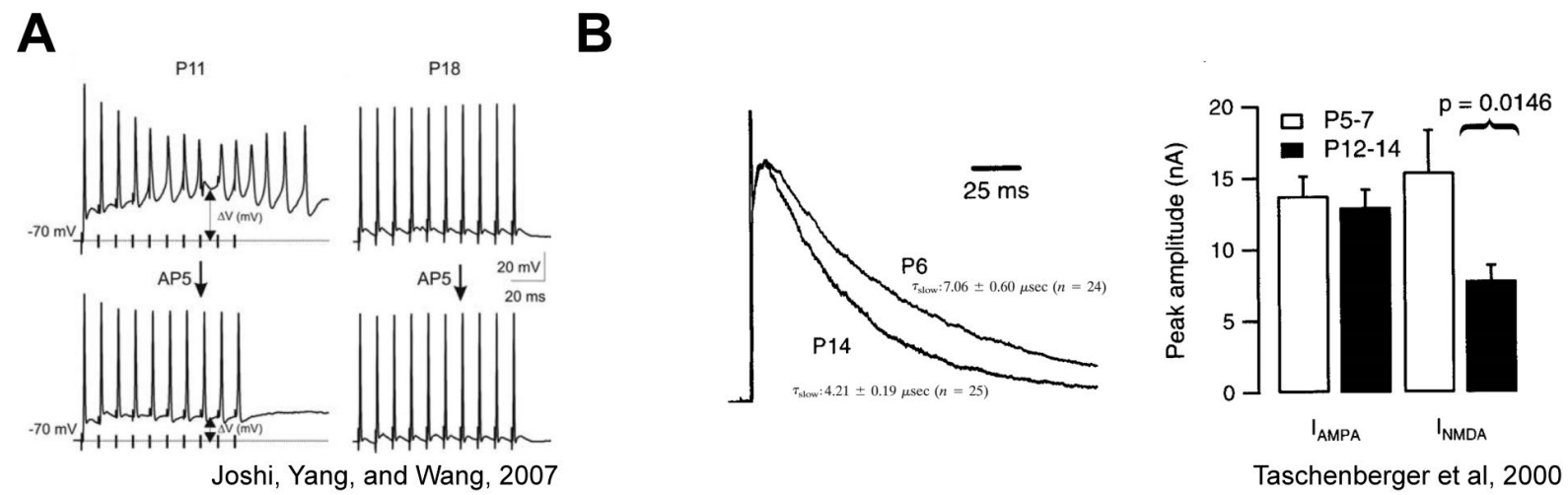

C

Pre- and Post- Pairing at $35^{\circ} \mathrm{C}$
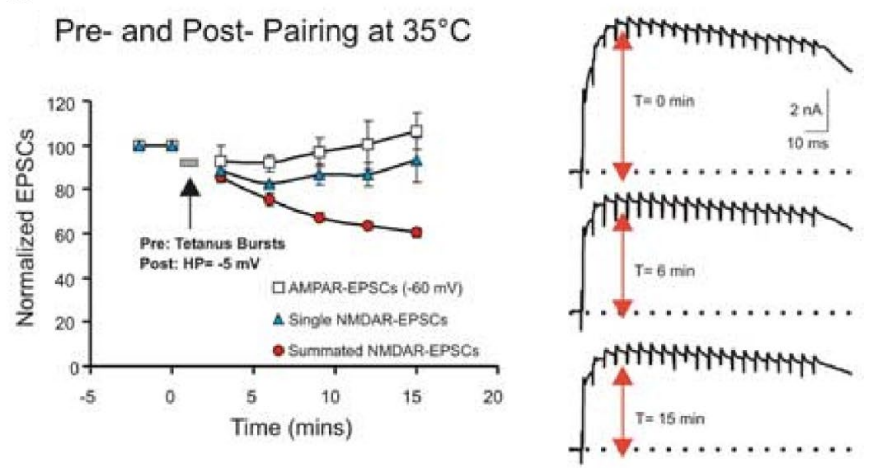

$D$ Mouse mRNA Protein

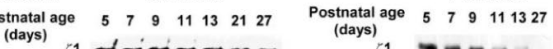
51 வ ع1/2 $-\infty-\infty$ इ1 $-\infty-\cdots$ $\varepsilon 1=-$

Futai et al, 2001

E $\quad R a$

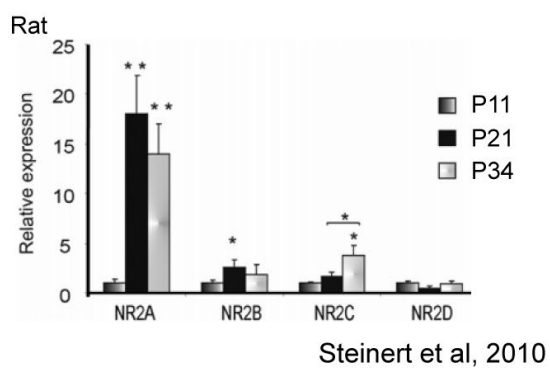

Figure 18: Changes in Principal Cell NMDA Channels During Development. A. Stimulation of $100 \mathrm{~Hz}$ to calyceal axons (black bars) causes summation of tail currents $(\triangle \mathrm{V})$ in EPSCs in P11 (top left) mice, while P18 mice (top right) show no summation. When treated with AP5 (selective NMDA antagonist), P11 cells show a more mature phenotype with little summation (bottom left), while EPSCs in P18 neurons were unaffected (bottom right). EPSCs were recorded in voltage clamp mode $(-70 \mathrm{mV})$. B. While AMPA receptor EPSC amplitude shows no change from P5-P14, both the decay time constant (Tslow, left) and amplitude (right) of the NMDAR-mediated current decrease over this time frame. C. After measuring EPSC response to $0.05 \mathrm{~Hz}$ presynaptic stimulation at a postsynaptic holding potential of $-60 \mathrm{mV}$ (first data point) and both a presynaptic $100 \mathrm{~Hz}$ burst and single stimulation at a $+60 \mathrm{mV}$ holding potential (second data point), a paired presynaptic stimulation and postsynaptic depolarization was sustained for 1 minute (grey bar). At two minutes after this stimulation/depolarization was stopped and at 4 additional 3 minute intervals, measurements of EPSC response to $100 \mathrm{~Hz}$ stimulus bursts (summated NMDAP-EPSCs, red) and single stimulations (AMPAR-EPSCs, white; Single NMDAR-EPSCs, blue) were recorded. While both AMPAREPSCs and individual NMDAR-EPSCs remain stable, a decrease in the summated NMDAR-EPSC is evident (red, left graph; right traces) suggesting a decrease in NMDARs. D. NMDAR subunit mRNA (left, RT-PCR

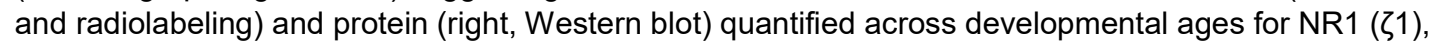
NR2A $(\varepsilon 1)$ and NR2B $(\varepsilon 2)$ in the mouse. While NR1 mRNA was consistently expressed, the protein product decreased across development. Both NR2A and NR2B mRNA and protein decreased as well, with NR2A protein decreasing earlier. The G3PDH (left) housekeeping gene was used as a control. E. mRNA expression for NMDAR subunits in the rat assessed by quantitative PCR. In contrast to findings in the mouse (panel D), expression of NR2A and NR2B mRNA increased from P11-21, and only decreased slightly from P21-34. Additionally, NR2C showed an increase from P21-34, while no significant change was found for NR2D. Adapted from Joshi, Yang, and Wang, 2007; Taschenberger et al, 2000; Futai et al, 2001; and Steinert et al, 2010. 

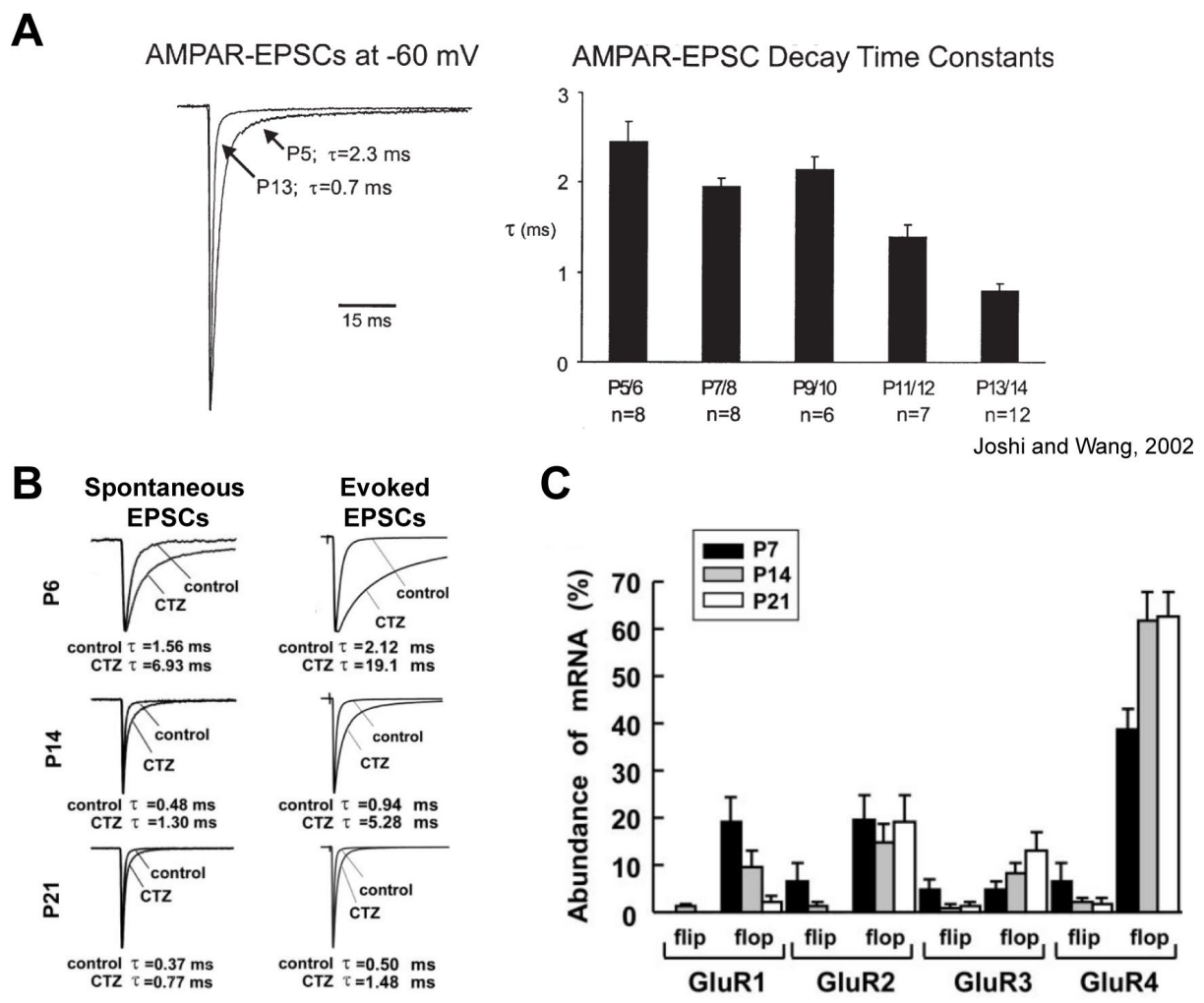

C

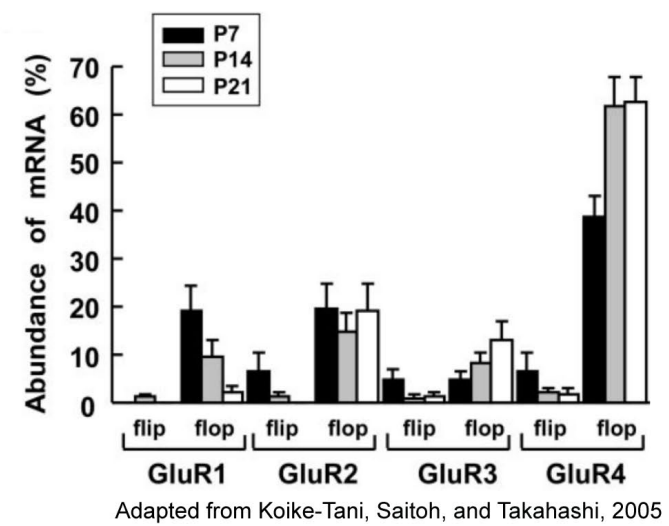

D
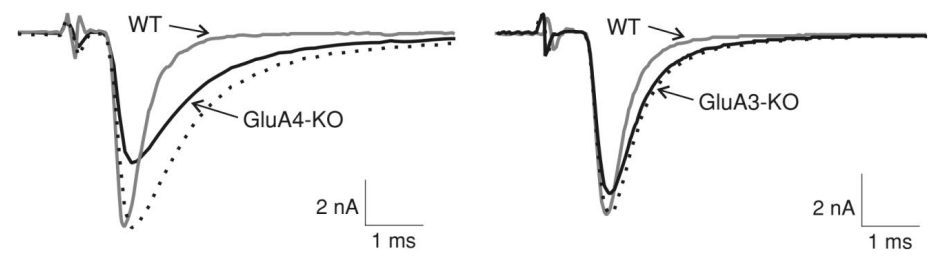

Yang et al, 2011

Figure 19: Changes in Principal Cell AMPA Channels During Development. A. AMPAR-mediated EPSCs become sharper during development in part due to a decrease in decay time (left). From P5-14, the AMPAR-EPSC decay time constant decreases by $\sim 70 \%$ (right). B. A comparison of spontaneous (left) and evoked (right) AMPAR-EPSCs (sEPSC and eEPSC, respectively) with and without CTZ, a positive allosteric modulator of AMPARs that decreases desensitization. CTZ has a developmentally decreasing influence on both sEPSCs and eEPSCs (compare control and CTZ time constants), and less influence on eEPSCs than sEPSCs most likely due to the higher glutamate release in eEPSCs (compare $\Delta$ TCTZ-control). C. Expression of AMPAR subunits (GluR1-4) from P7-14 in the rat. GluR1 decreases during development, while GluR3 and GluR4 increase and GluR2 shows no significant change."Flip" isoforms are significantly less prevalent at all ages than "flop" isoforms. E. AMPAR-EPSCs in GluR4 (GluA4) knockout mice at P16-19 show significantly increased rise and decay times and decreased amplitudes compared to wild-type (left). Although there is a significant difference in decay time in GluR3 (GluA3) knockouts, no other features of the EPSC are significantly disrupted (right). Adapted from Joshi and Wang, 2002; Koike-Tani, Saitoh, and Takahashi, 2005; and Yang et al, 2011. 
A
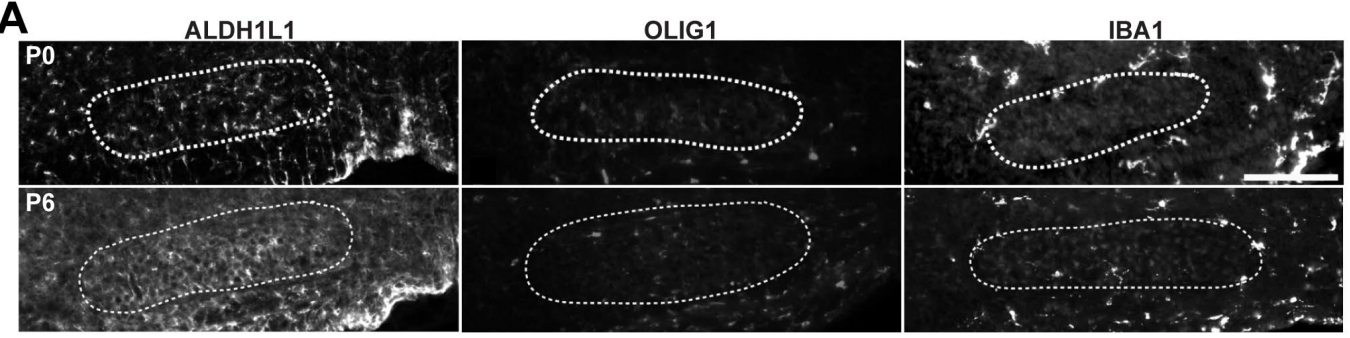

B
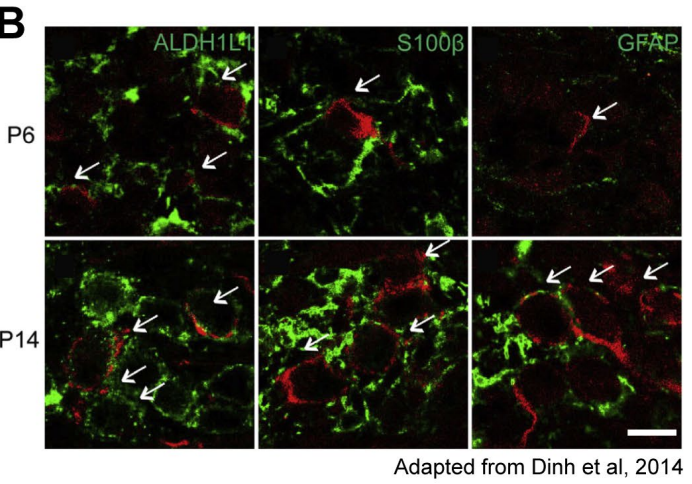

C

Adapted from Dinh et al, 2014

D
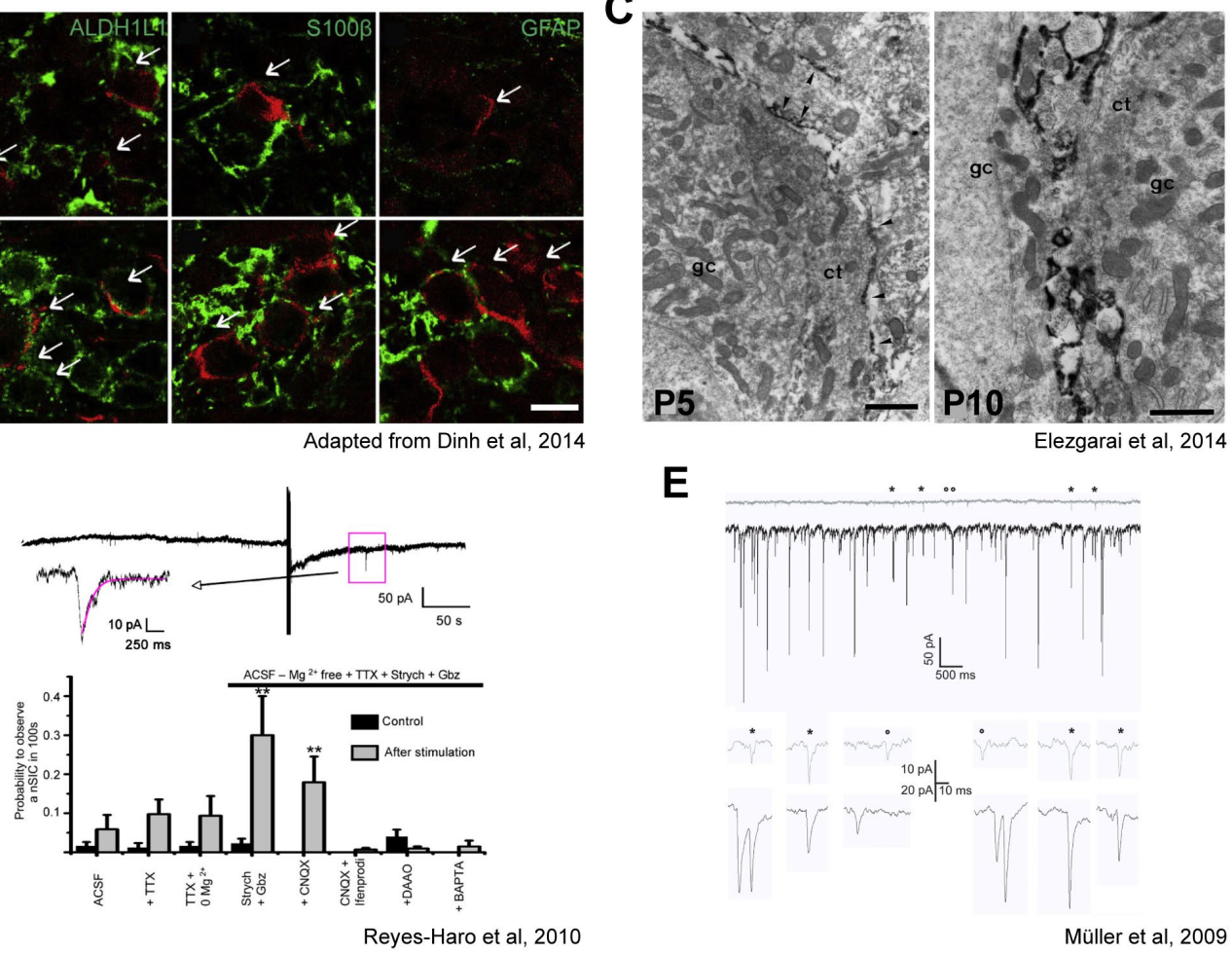

$\mathbf{E}$

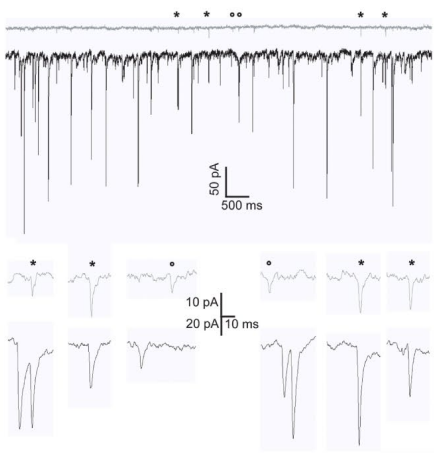

Müller et al, 2009

Figure 20: Glial Development and Association with the CoH:MNTB Connection in MNTB. A. Astrocytic (ALDH1L1, left) and oligodendrocytic (OLIG2, middle) processes are present in the MNTB as early as P0, and increase in density by P6. ALDH1L1 processes appear to surround cell bodies at both ages. Microglia (IBA1, right), though present in surrounding brain regions, are absent from the MNTB at P0 and only

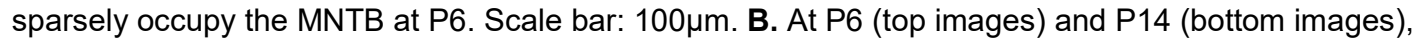
ALDH1L1-positive (left, green) and S100ß-positive (middle, green) astrocytic processes are in close apposition to both the principal cells (void inside round process profiles) and the calyx of Held (red, arrows). GFAP-processes (right, green) are largely absent from the MNTB at P6, and not associated with the calyx. However, by P14 these processes are more prevalent and associated with the calyx (bottom right). Scale bar: $25 \mu \mathrm{m}$. C. Immunoperoxidase reaction shows metabotropic glutamate receptors $2 / 3$ (mGluR2/3, black and arrowheads) within glial processes in apposition to both calyceal terminals (ct) and principal cells (gc) at P5 and P10. Scale bar: 1 $1 \mu \mathrm{m}$. D. Example of a neuronal slow inward current (nSIC) present following an electrical stimulation-induced, astrocyte-activating calcium wave (top). Through a combination of receptor inhibition (TTX, strychnine, gabazine) and decrease in NMDA Mg ${ }^{2+}$ block, the prevalence of $n S I C s$ can be increased. As this increase can be countered by CNQX and ifenprodil (NMDA antagonists) and DAAO (degrades D-serine), the neurotransmitters released by astrocytes to mediate nSICs can be identified as glutamate and D-serine. E. Simultaneous recording of spontaneous postsynaptic currents from NG2+ glia (top trace) and principal cells (bottom trace) reveals both coincident (asterisks) and non-coincident (circles) events. Below are expanded traces for each event. Adapted from Dinh et al, 2014; Elezgarai et al, 2014; Reyes-Haro et al, 2010; and Müller et al, 2009. 
A

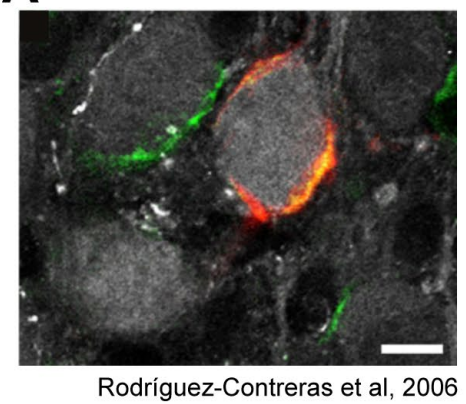

B

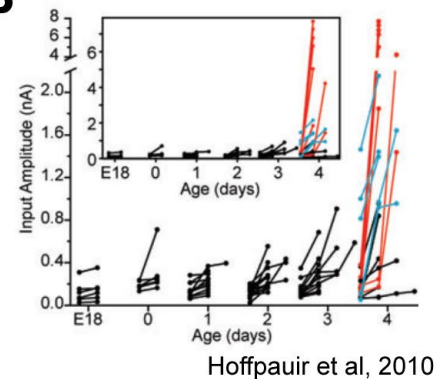

D

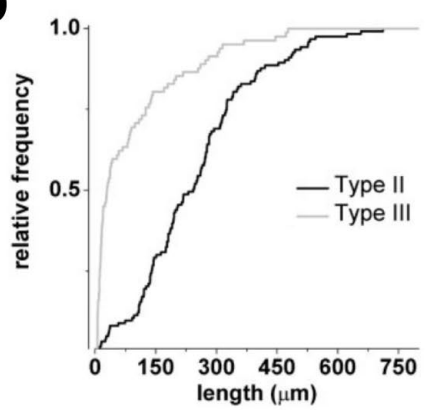

Rodríguez-Contreras et al, 2006
C
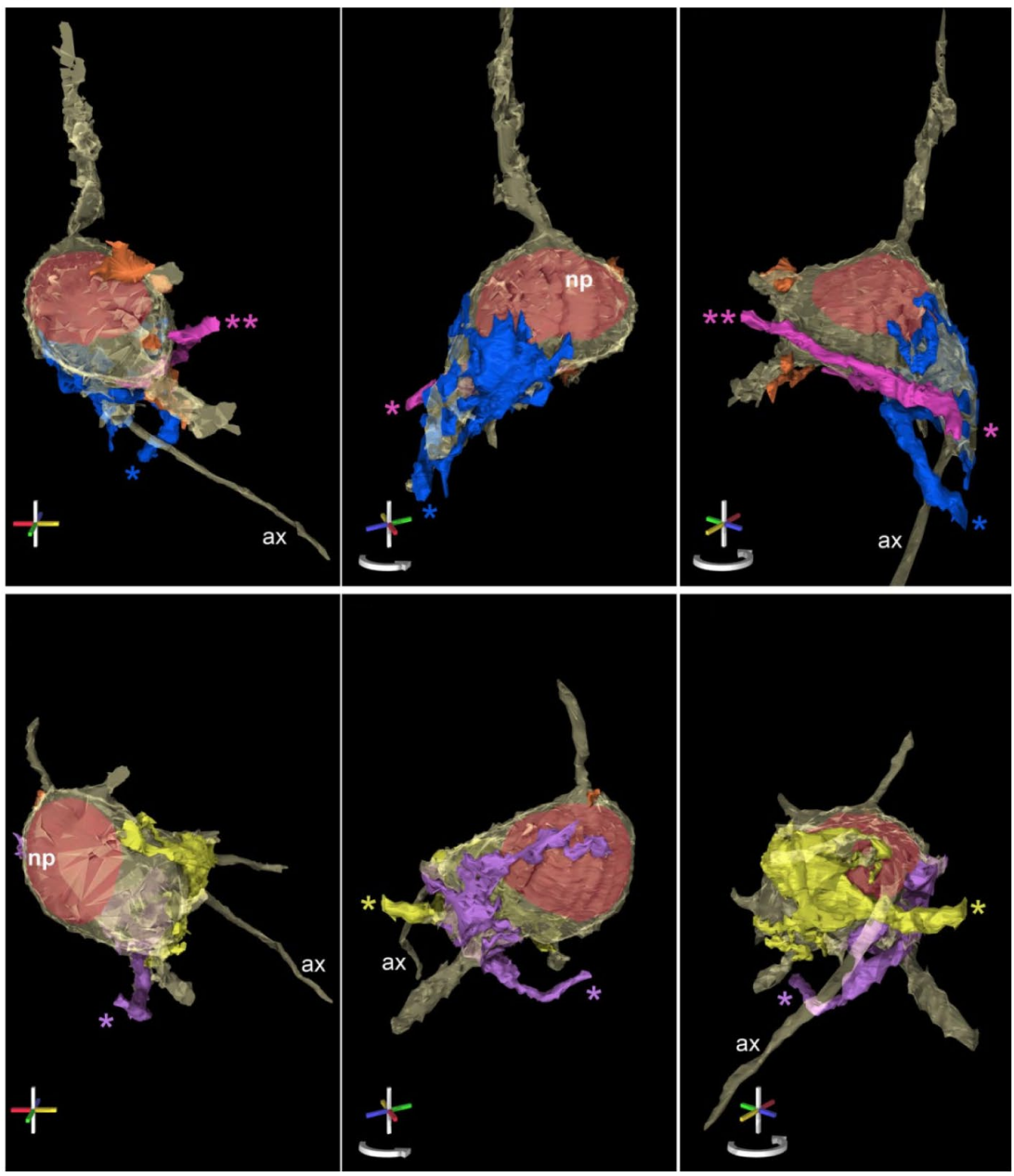

Hoffpauir et al, 2006

Figure 21: Competition During Calyceal Development and Principal Cell Polarity. A. Principal cells (PCs) in the P9 rat were visualized with anti-calbindin (grey), while calyceal terminals were stained with antiVGLUT1/2 (green). A fluorescent conjugated dextran amine-labeled axon (red) overlaps completely with one large terminal (orange). However, several other terminals in the image do not have associated axons, and thus remain green. B. Minimal stimulation protocol reveals multiple inputs on PCs from E18-P4. Circles represent unique inputs and lines represent terminals present on a single PC. Cells possessing terminals with similar, small amplitudes are in black, those with larger amplitude terminals (0.8-2.2nA) but whose terminals were within $0.75 \mathrm{nA}$ of one another (potential competitors) are in blue, and cells with one terminal (probable protocalyx) at least $1 \mathrm{nA}$ in amplitude larger than the next largest input are in red. C. 3D reconstructions from serial section electron micrographs of two P4 neurons (tan; top row and bottom row are each one cell), each with eccentric nuclei (red) and two large terminals (blue and cyan on top, purple and yellow on bottom). Middle and right images depict cells in the left images rotated counterclockwise $120^{\circ}$ (middle) and $240^{\circ}$ (right). ax - principal cell axon, $\mathrm{np}$ - nuclear pole. D. Prevalence of Type II (branched with only one calyx) and Type III (branched, calyx formed on each branch) with respect to the distance of their branch point from the calyx. Axons producing multiple calyces tend to branch close to the location of the terminals. Adapted from Rodríguez-Contreras et al, 2006; Hoffpauir et al, 2006 and 2010. 
A
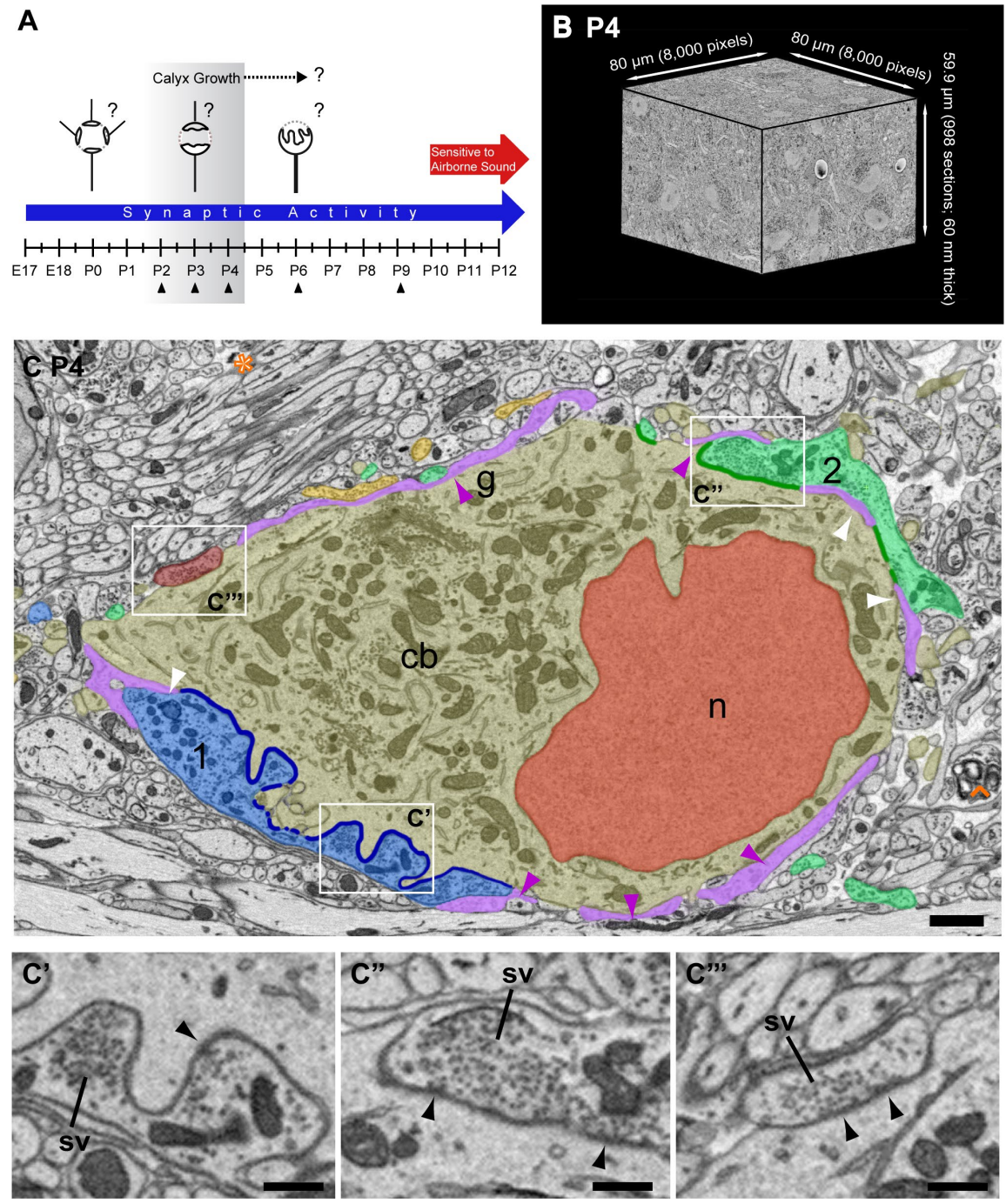

Figure 22: Experimental design and tissue analysis for time series SBEM. A, Calyx growth occurs in context of synaptic activity and precedes ear canal opening (termed sensitivity to airborne sound). Tissue samples taken at postnatal (P) ages (P2, P3, P4, P6, P9) to permit assay of exuberant innervation, strengthening and pruning of a subset of inputs and resolution of mono-innervation (question marks) and dynamics of calyx growth (shaded area, calyx growth $\square$ ?). B, Representative SBEM volume from P4 animal. Cross-sectioned neurons, glia and capillaries (white circles with darkened cores) are evident on the block faces. Perspective view alters perceived length of edges. C, Portion of single slice from SBEM volume depicted in panel B reveals cellular details. Objects segmented from volume by tracing cell membranes are colorized for ease of identification in this figure: nucleus ( $\mathrm{n}$, red) ; cell body (cb; gold); largest calyciform input (1, blue); second largest calyciform input (2, green); smaller inputs (burgundy, yellow); glial covering of cell body (g, purple colored and purple arrowheads) and thin glial incursions between input and cell body (white arrowheads). Yellow input is excluded by glia from contact with soma in this section. Size of inputs quantified as apposed surface area with cell body (dark blue line on input 1 , dark green line on input 2), excluding glial incursions. Developing brain contains much open space (orange asterisk) and areas of degeneration (orange caret). Zoomed views of three inputs (C',C",C"') show groupings of synaptic vesicles (sv) and active zones (black arrows) where vesicles cluster along the presynaptic membrane. Scale bar: $2 \mu \mathrm{m}$ in panel C, $0.5 \mu \mathrm{m}$ in panels C'-C'”. 

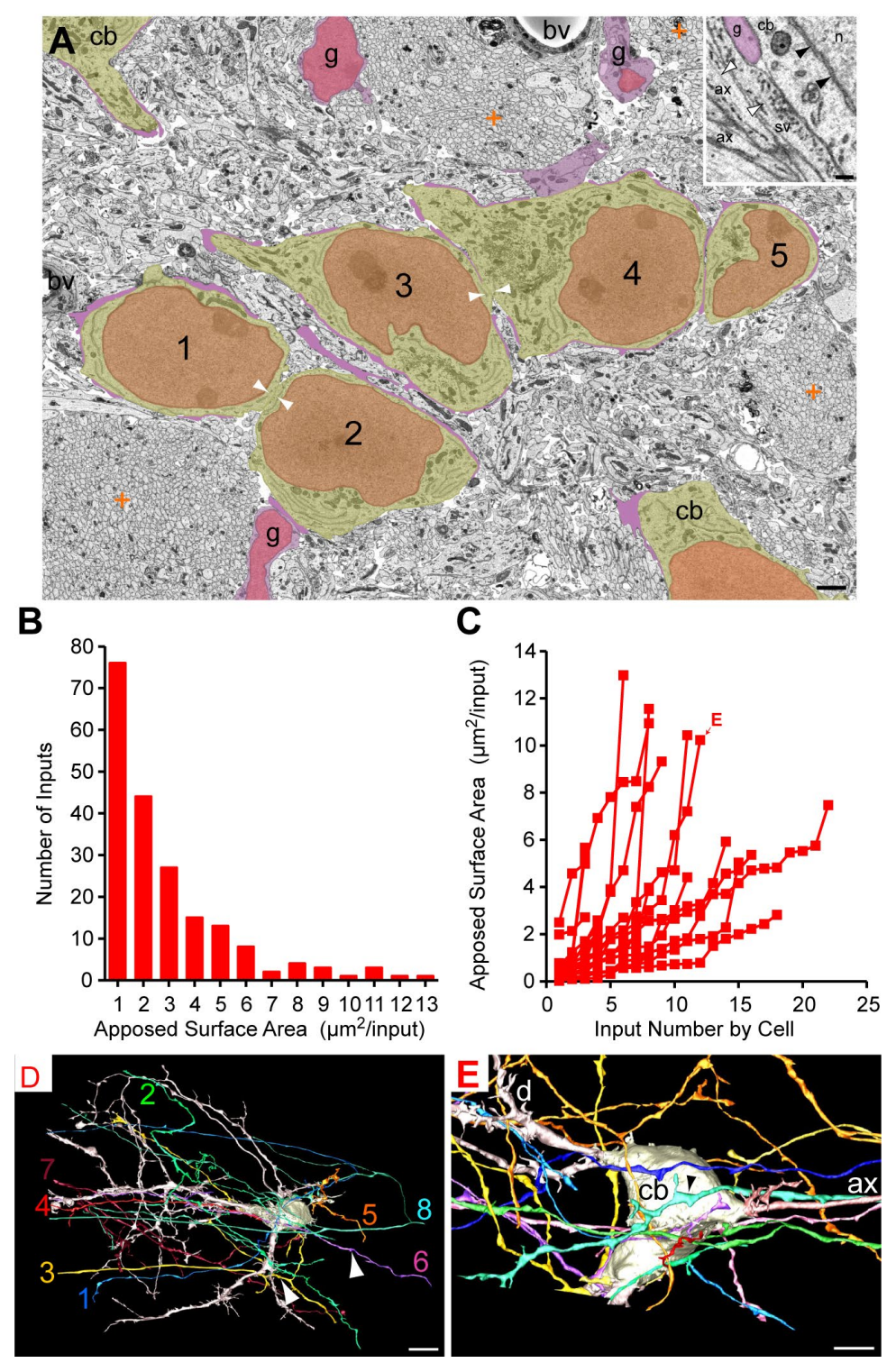

Figure 23: Clustering of cells and quantification of somatic inputs at P2. A, Region of single image at P2 shows cluster of MNTB cells (gold, numbered 1-5) with direct membrane apposition (white arrowheads) and glial covering (purple) which can form thin layers between cells. Glial cells (g), other MNTB cell bodies (cb) and blood vessel (bv) also shown. Large bundles of crosscut cellular processes (orange plus signs) are prominent structures. Inset, Example of small synaptic terminal identified by synaptic vesicles (sv) clustered at apposition with thin region of MNTB cell body cytoplasm (cb) surrounding nucleus ( $n$; black arrowheads indicate double membrane of nuclear envelope). Microtubules (white arrowheads) are evident in axon forming synaptic contact and adjacent axons (ax) which do not contain synaptic vesicles. B, Size distribution (apposed surface area) of all inputs $(n=204)$ to reconstructed cells $\left(21 / 71\right.$ cells in volume), binned at $1 \mu m^{2}$ increments. C, Inputs (red squares) to each cell linked by lines and plotted from smallest to largest. Cell in panel $E$ is indicated by "E" and arrow. D, 3D reconstruction of cell with small inputs to cell body (numbered 1-8) and showing highly branched dendrites. Several inputs branch from medial-lateral oriented axons $(3,8)$. E, Close-up of another MNTB neuron showing cell body (cb), proximal dendrites (d), axon (ax) and inputs to cb. One of the largest inputs at this age makes en passant contact (black arrowhead). Dendrite and axons truncated at bottom by edge of image volume. Scale bars: $2 \mu \mathrm{m}$ in panel A; $0.5 \mu \mathrm{m}$ in panel A inset; $10 \mu \mathrm{m}$ in panel D; $5 \mu \mathrm{m}$ in panel $\mathrm{E}$. 

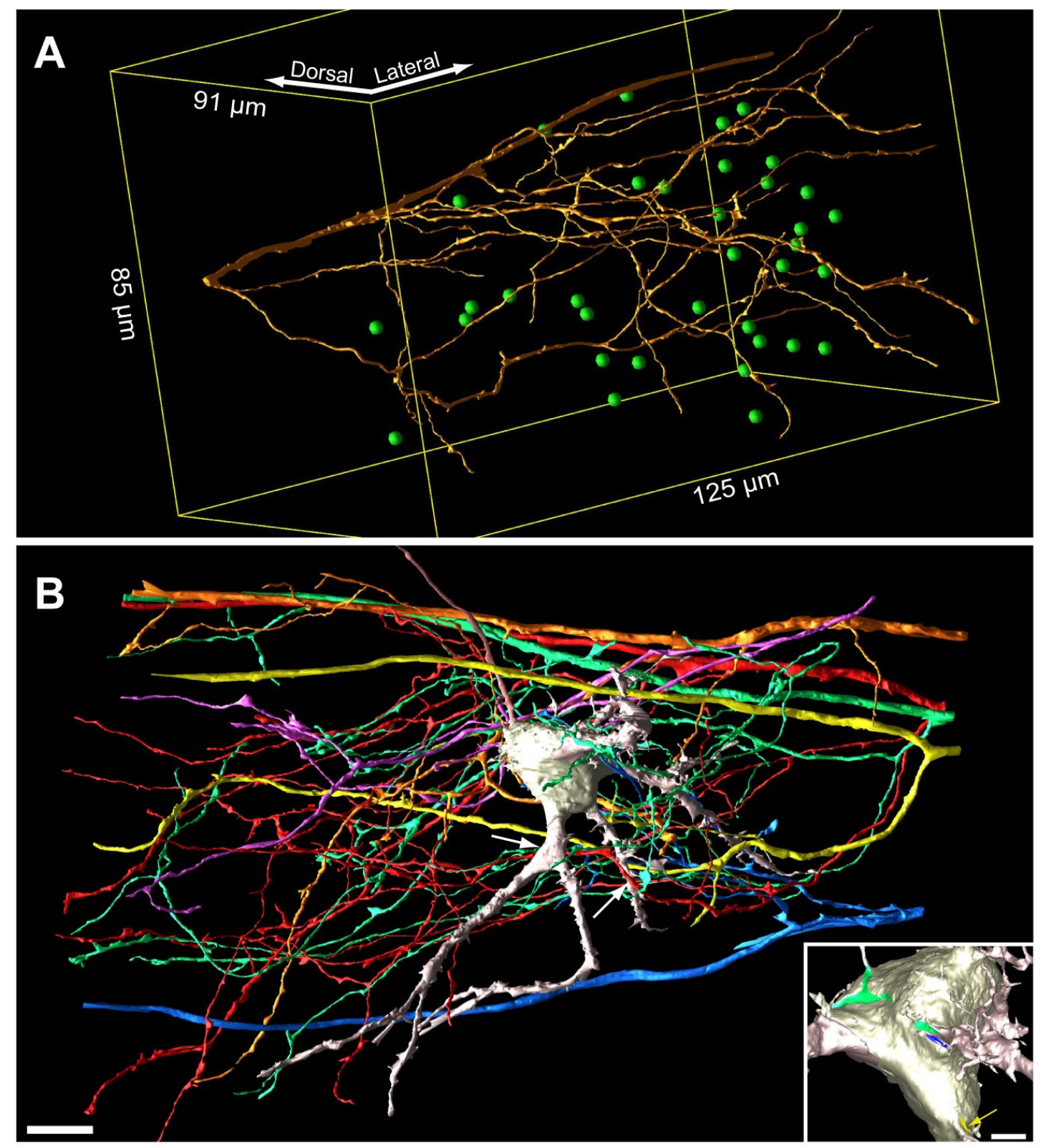

Figure 24: Exuberant innervation of MNTB at P2. A, Reconstruction of single large diameter axon oriented medially-laterally in image volume. Green spheres indicate center of MNTB cells (35/71 cells in volume) with synaptic contact from this axon onto soma or dendrites. Yellow lines depict outline of image volume. B, To illustrate convergence of multiple inputs, a cell contacted by five large diameter trapezoid body axons on dendrites (white arrows; left arrow indicates contact on opposite side of dendrite) or cell body (green, blue, and yellow (arrow highlights ASA) in inset; fourth ASA not shown) is shown. Contacts occurred via branches from parent axon. Scale bar: $10 \mu \mathrm{m}$ in panel B, $2.5 \mu \mathrm{m}$ in panel B inset. 

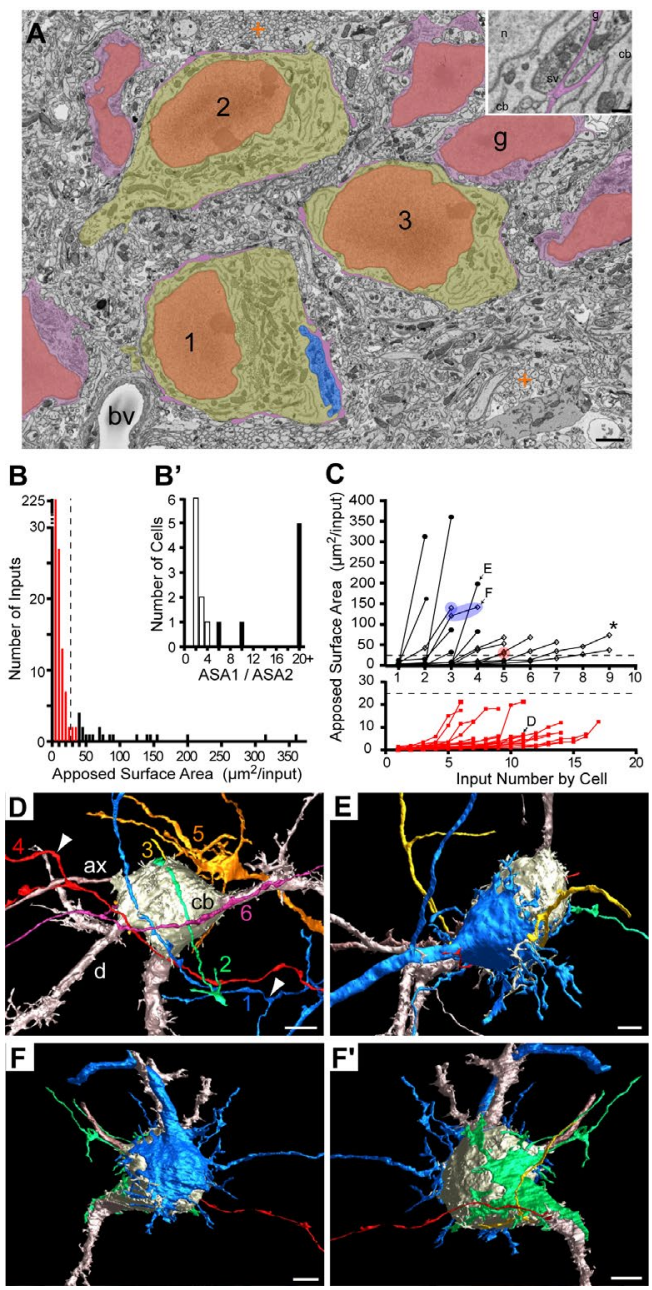

Figure 25: Separation of cells and beginning of rapid nerve terminal growth at $\mathrm{P} 3$. $A$, Region of single image showing small field of cells (numbered 1-3) with small inputs and less cell-cell contact than at P2, yet retaining significant coverage of cell body surface by glia (purple). A large input (blue) contacts cell 1. Color scheme same as figure 2. Crosscut cellular processes (orange plus signs) indicated as in figure 2. Inset, Inputs to two adjacent cell bodies (cb) identified by synaptic vesicles (sv) and mitochondria and covered by glial process (g, purple) $B$, Size distribution (ASA) of all inputs $(n=298)$ onto reconstructed cells ( $37 / 37$ cells in volume), binned at $5 \mu \mathrm{m}^{2}$. Local minimum at $25 \mu \mathrm{m}^{2}$ (vertical dashed line) is operational distinction between small (red) and large inputs (black). $B^{\prime}$, Inputs to cells with at least one large input (16/37 cells) were analyzed for relative size of two largest inputs (size ratio $=$ ASA1/ASA2). Cells with ASA1/ASA2 $>5$ (7/16 cells, closed histogram bars) formed a distinct population from those with smaller size ratios (9/16 cells, open black bars). $\boldsymbol{C}$, Inputs (closed black circles, open black diamonds, red squares) to each cell linked by lines and plotted from smallest to largest. Cells had 1 (e.g. open black diamond inside blue or red circle), mostly 2 or 3 (asterisk) large inputs and both inputs could exceed $100 \mu \mathrm{m}^{2}$ (blue ellipsoid, labeled F). Cells with only small inputs (red) graphed separately for clarity. $\boldsymbol{D}, 3 \mathrm{D}$ rendering of representative cell contacted by only small inputs. Input 5 is branched from a larger nerve terminal growing on an adjacent cell body. Sizes of inputs to this cell shown in panel $C$ and marked " $D$ ". Only largest 6 of 11 inputs shown for clarity. E, 3D rendering of representative cell with a dominant large input (blue). Sizes of inputs to this cell shown in panel $C$ and marked "E". $\boldsymbol{F}$ and $\boldsymbol{F}$ ', 3D rendering of cell showing largest (blue) and second largest (green, rotated view of same cell) inputs, both of which were $>100 \mu \mathrm{m}^{2}$. Sizes of inputs to this cell shown in panel $C$ and marked " $F$ ". Abbreviations: cb, cell body; $d$, dendrite; ax, axon. Scale bars: $2 \mu \mathrm{m}$ in panel A; $0.5 \mu \mathrm{m}$ in panel A inset; $5 \mu \mathrm{m}$ in panels $D-F$ '. 


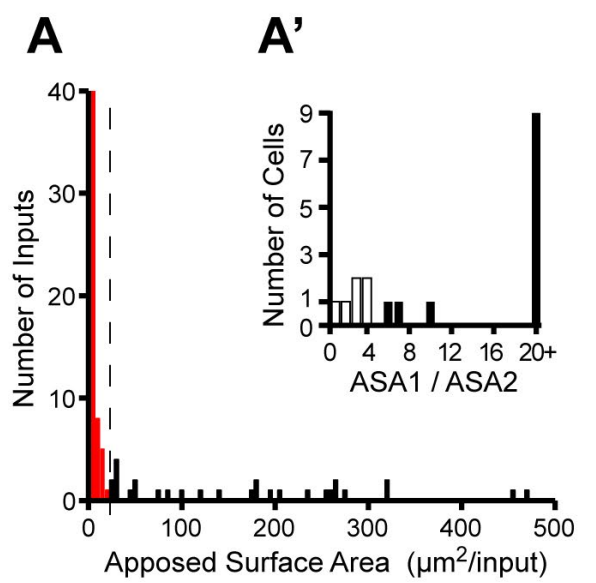

B
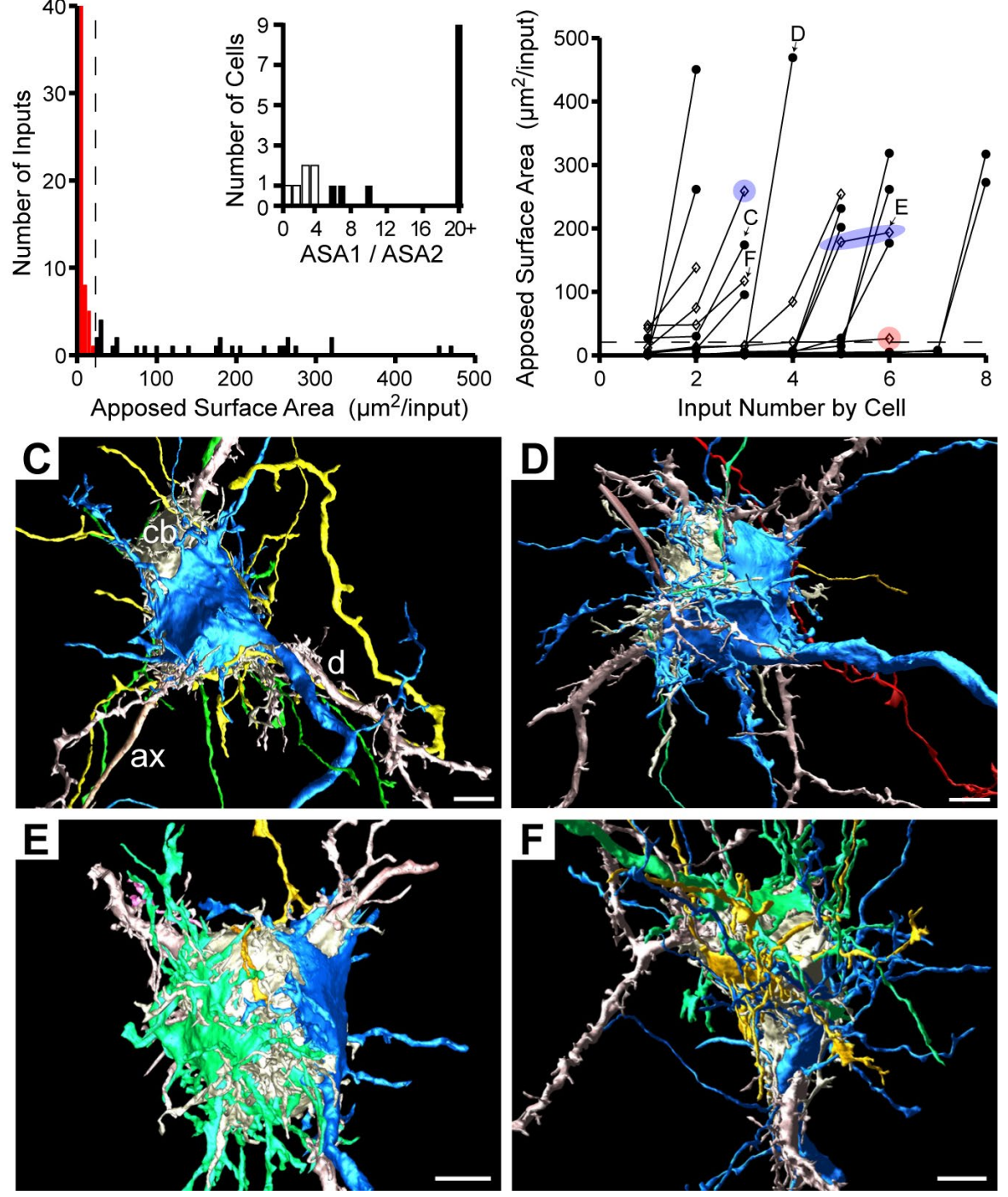

Figure 26: All cells contacted by large terminals at P4. A, Size distribution (ASA) of all inputs $(n=83)$ onto reconstructed cells (18/18 cells in volume), binned at $5 \mu \mathrm{m}^{2}$. Local minimum at $25 \mu \mathrm{m}^{2}$ (vertical dashed line) distinguishes small (red) and large inputs (black). A', Inputs to cells with at least one large input (18/18 cells) were analyzed for their size ratio (ASA1/ASA2). Cells with size ratio $>5(12 / 18$ cells, solid black histogram bars and solid black circles in panel $B$ ) formed a distinct population from those with smaller size ratios (6/18 cells, open black bars and open black diamonds in panel B). B, Inputs (closed black circles, open black diamonds) to each cell linked by lines and plotted from smallest to largest. Largest inputs $\left(>260 \mu \mathrm{m}^{2}\right)$ were always dominant inputs (size ratio $>5$ ). Cells with actively competing inputs (size ratio $<5$ ) had 1 (e.g., open black diamond in red circle), mostly 2 (e.g., open black diamond in blue circle) or 3 (labeled F) large inputs. Two competing inputs could each exceed $150 \mu \mathrm{m}^{2}$ (blue ellipsoid, E). C, Cell with a dominant large input (blue; sizes of inputs to this cell indicated by "C" in panel B). D, Cell with largest input (blue; sizes of inputs to this cell indicated by "D" in panel B). E, Cell with two large competing inputs (blue, green; sizes of inputs to this cell indicated by "E" in panel B). Two smallest inputs to the cell were omitted from the image for clarity. F, Cell with three large competing inputs (blue, green, yellow; sizes of inputs to this cell indicated by "F" in panel B). Abbreviations: cb, cell body; $d$, dendrite; ax, axon. Scale bars: $5 \mu \mathrm{m}$. 

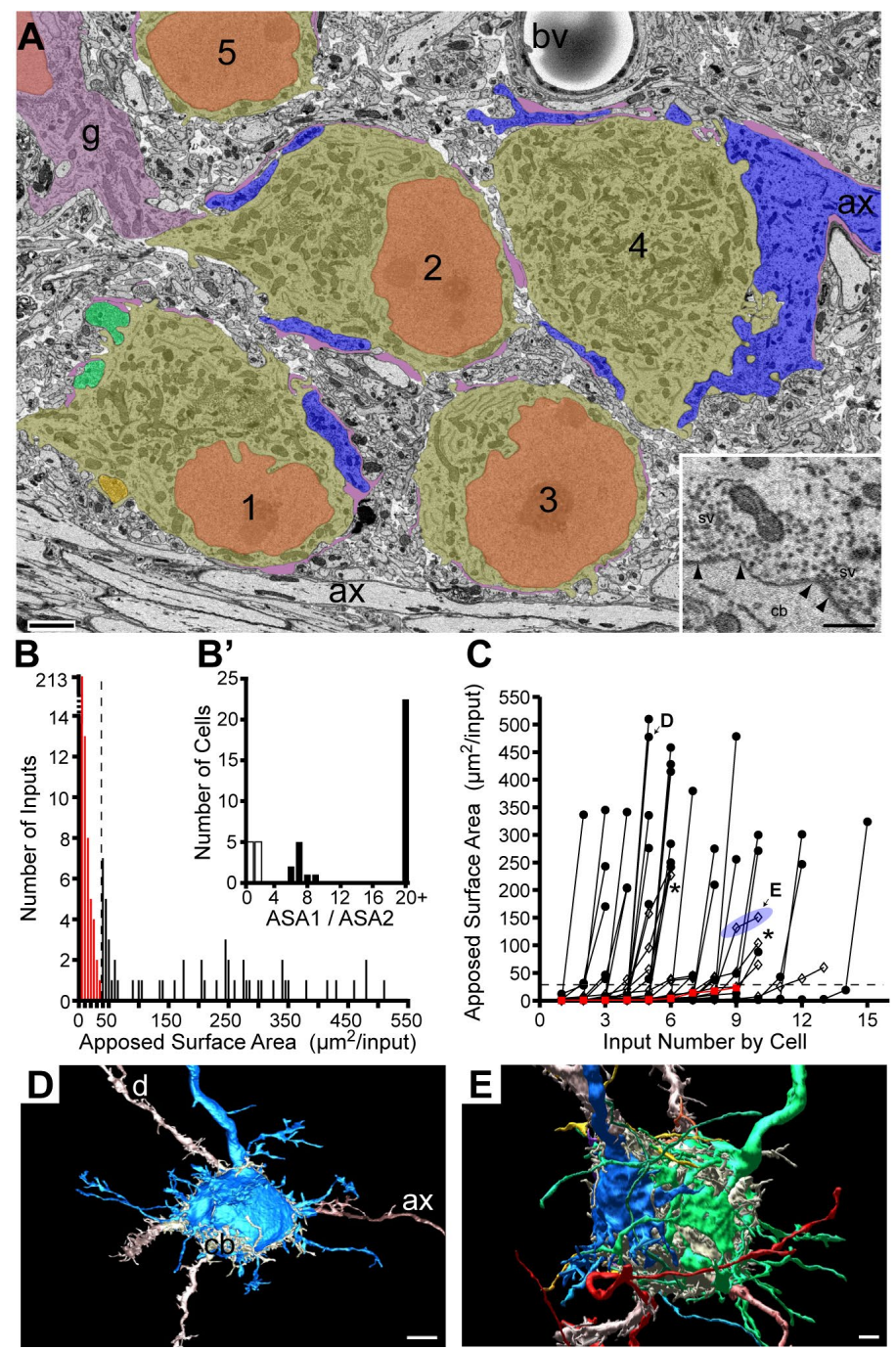

Figure 27: Continued growth and competition at P6. A, Region of single image showing small field of neurons with dominant large (cells 2-5; large inputs to cells 3 and 5 not captured in this section) and actively competing inputs ( 3 inputs shown onto cell 1 ) and a glial (g) cell body. Axon forming calyx onto cell 4 enters at right edge of image. Glial laminae (purple) cover portions of cell body but also the non-synaptic surface of calyces and calycigenic inputs. Color scheme same as figure 4A. Fiber fascicle traverses in medial-lateral direction at bottom of image. A Inset, Terminals contain visible active zones defined by synaptic vesicles (sv) clustered along the presynaptic membrane (black arrowheads). B, Size distribution (ASA) of all inputs $(n=306)$ onto reconstructed cells ( $43 / 43$ cells in volume), binned at $5 \mu \mathrm{m}^{2}$ increments. Local minimum at $35 \mu \mathrm{m}^{2}$ (vertical dashed line) distinguishes small (red) and large inputs (black) that potentially form the single calyceal input. B', Inputs to cells with at least one large input (42/43 cells) were analyzed for size ratio (ASA1/ASA2). Cells with size ratio $>5$ (32 cells, solid black histogram bars) formed a distinct population from those with smaller size ratios (10 cells, open black bars). C, Inputs (solid black circles, open black diamonds, red squares) to each cell linked by lines and plotted from smallest to largest. Largest inputs $\left(>250 \mu \mathrm{m}^{2}\right)$ were always dominant inputs (size ratio $\left.>5\right)$. Cells with actively competing inputs (size ratio $<5$ ) had 2 or 3 (asterisks) large inputs. Two competing inputs could each exceed $125 \mu^{2}$ (blue ellipsoid, labeled E). D, Cell with a dominant large input (blue; sizes of inputs to this cell indicated by " $D$ " in panel C). Smaller inputs omitted for clarity. E, Cell with two large competing inputs (blue, green; sizes of inputs to this cell indicated by "E" in panel C). Most smaller inputs omitted for clarity.

Abbreviations: cb, cell body; d, dendrite; ax, axon; bv, blood vessel. Scale bars: $2 \mu \mathrm{m}$ in panel A; $0.5 \mu \mathrm{m}$ in panel A inset; $5 \mu \mathrm{m}$ in panel $\mathrm{D} ; 2 \mu \mathrm{m}$ in panel $\mathrm{E}$. 

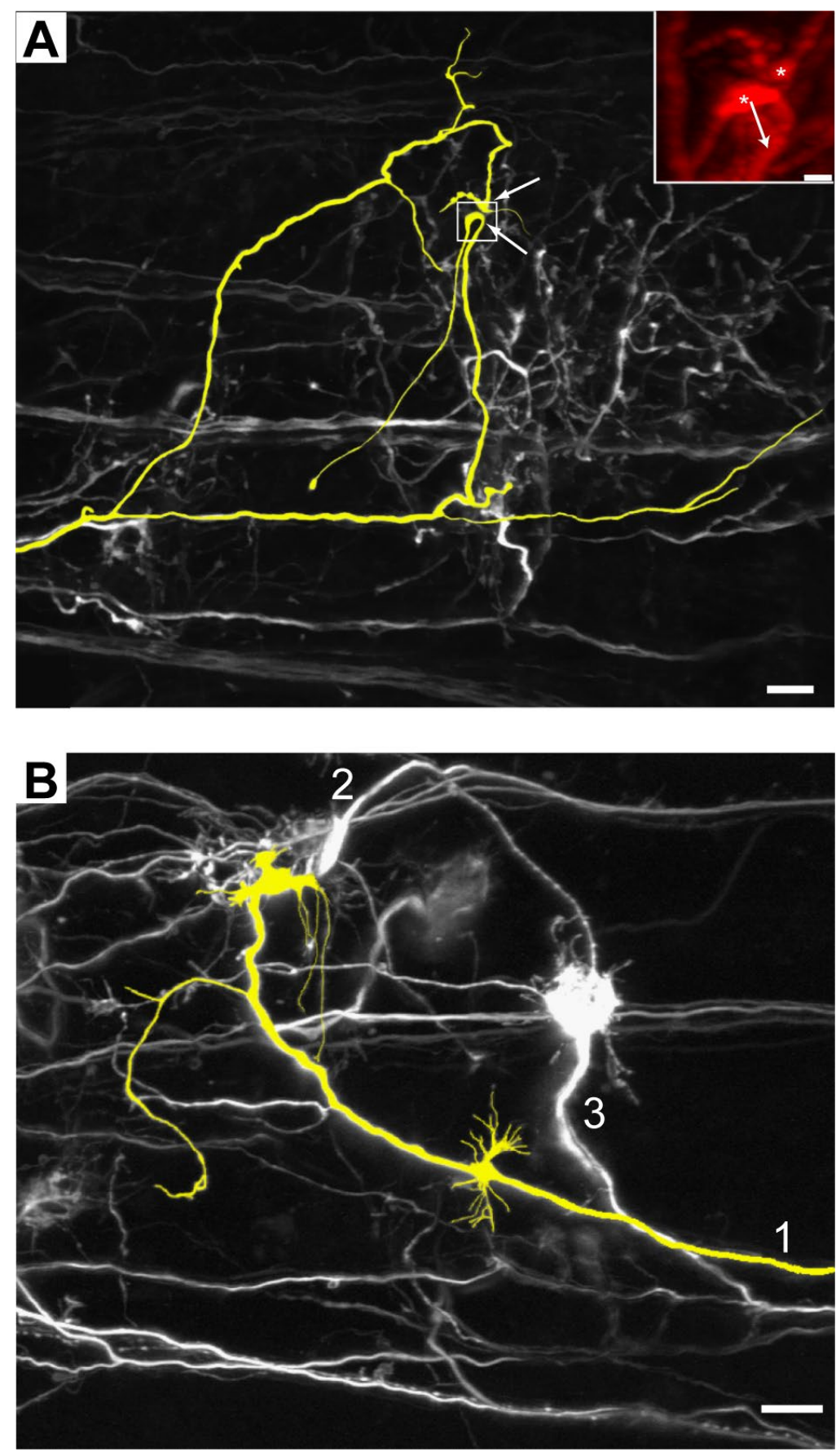

Figure 28: Branches of parent axon do not converge onto the same MNTB cell. A, Subset of axons electroporated with Alexa Fluor 488 dye and imaged using 2 photon microscopy in P3 animal. Images in both panels are of the collapsed image stack. Only example of axon and branches that terminate near each other (colorized yellow; white arrows indicate terminals). Inset, Close-up of boxed region viewed at angle to demonstrate curvature of large ending (arrow) away from smaller ending (both indicated with asterisks), likely indicating innervation of different postsynaptic structures. B, Typical pattern of branching and innervation where axon did not branch at medial locations (area not shown to right of panel B) and branches terminate in distinct regions in the MNTB. Axons 1 and 2 terminate via large endings near each other (P4 animal). Scale bars: $20 \mu \mathrm{m}$ in panels $A$ and $B ; 5 \mu \mathrm{m}$ in panel $A$ inset. 
A

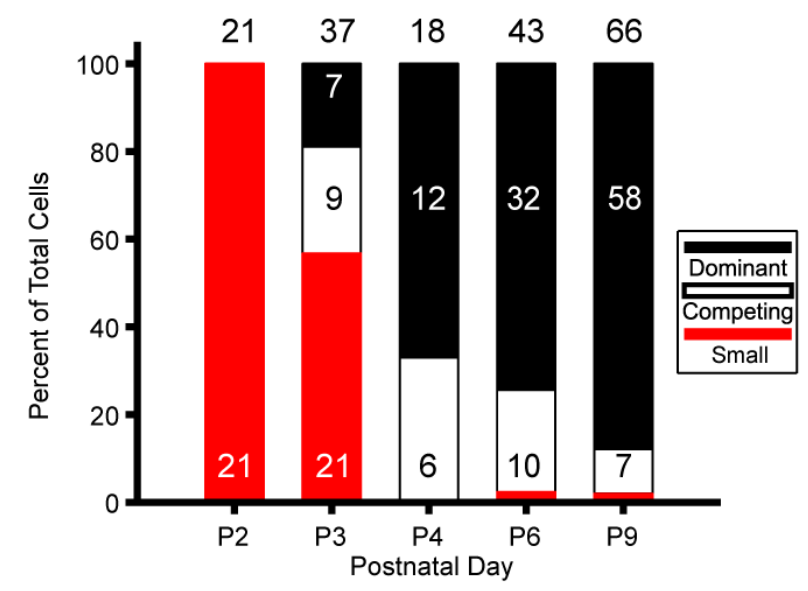

C

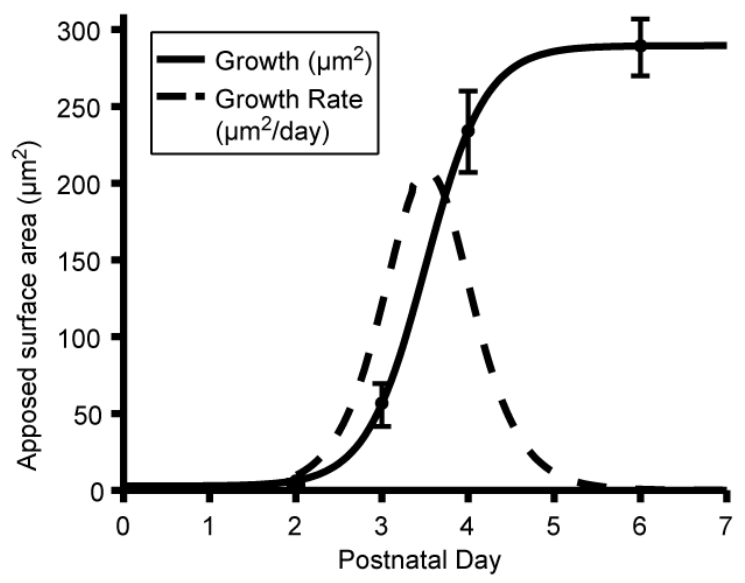

B

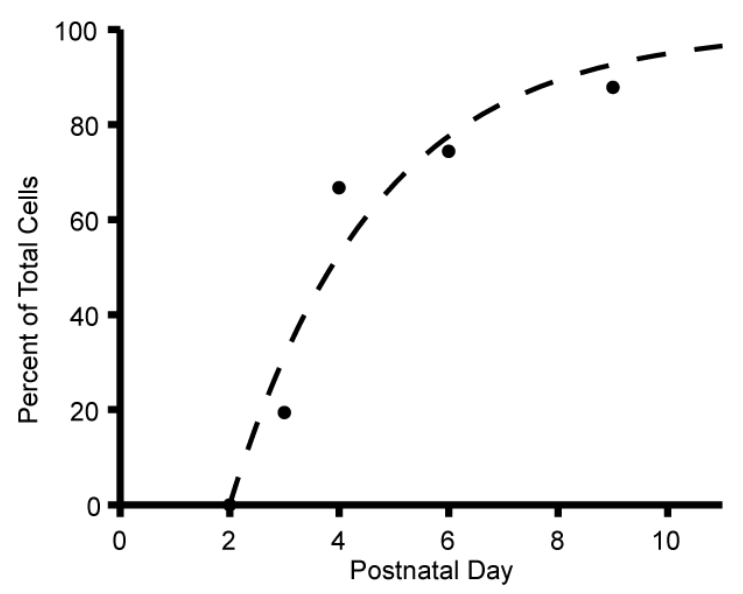

D

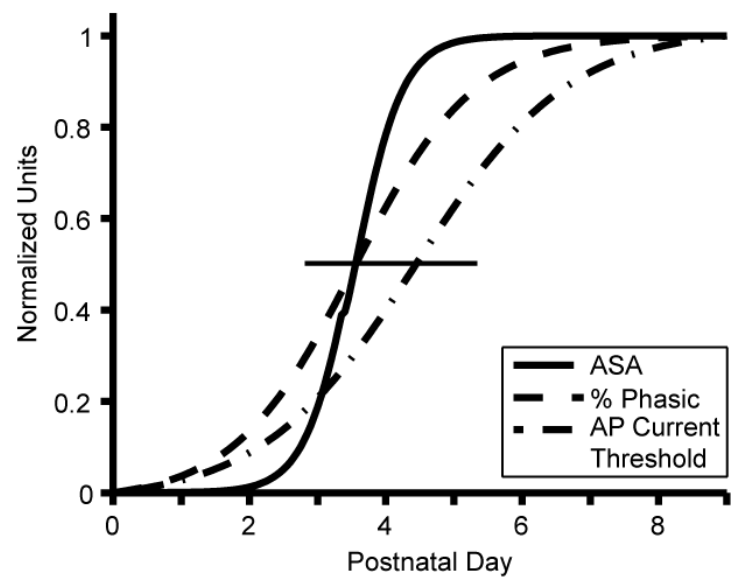

Figure 29: Competition and population growth dynamics of calycigenic terminals. A, Summary histograms of nerve terminal growth by prevalence of MNTB cells with inputs in the categories of (1) only small terminals (red), (2) actively competing large terminals (open; size ratio < 5) and (3) a dominant large input (black, size ratio $>5$ ). Numbers of cells analyzed at each age indicated above histograms. B, Dynamics for emergence of a dominant large input. Exponential fit with $\tau=2.7$ days, $R^{2}=0.94$. C, Population growth dynamics for calyxforming inputs. Data points are average of the largest terminal onto each reconstructed cell between P2-6; size of largest input at P9 was assessed qualitatively for most cells. At P6, cells with inputs $<75 \mu \mathrm{m}^{2}$ excluded from analysis as these likely formed a distinct population (see Results for P6 and P9). Data were fitted to a Boltzmann function as described in Methods. Slope of curve reveals growth rate, which peaks at $207 \mu \mathrm{m}^{2} /$ day. D, Coordinated time scales for calyx growth (Boltzmann fit from panel C, solid line), transition of action potential firing phenotype from tonic to phasic (dashed line) and increase in AP threshold to current pulse injection (dashdotted line) from Hoffpauir et al. (2010). Horizontal line indicates half-maximum of normalized ordinate values. 

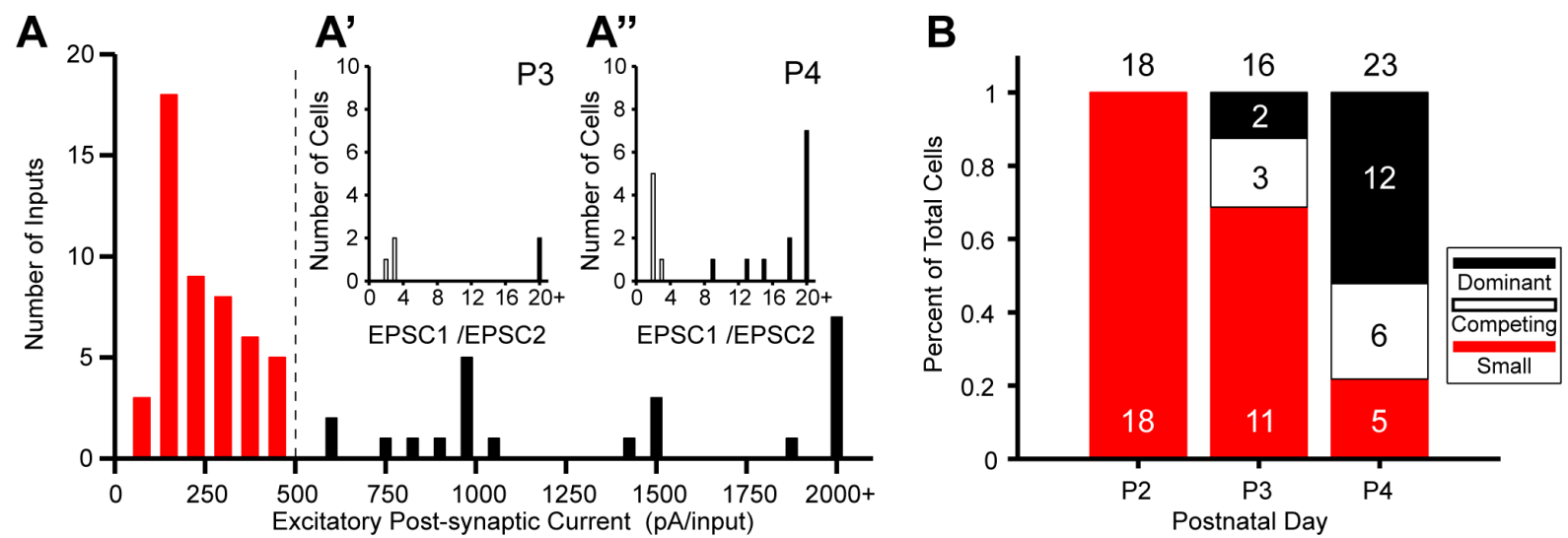

Figure 30: Electrophysiology methods underestimate innervation. A, Distribution of synaptic currents induced using minimal stimulation paradigm at P3 and P4, re-analyzed from Hoffpauir et al. (2010). Dashed line indicates break in histogram at 500 pA. A', A"', Input size ratio (largest input (EPSC1)/ second largest input (EPSC2)) shows groups of inputs with values $<5$ (open histogram bars in these panels and panel $B$ ) and $>5$ (black histogram bars in these panels and panel B). B, Dynamics for emergence of dominant large input, analyzed as for anatomical inputs in figure 8A. Number of cells at each age indicated above histogram. 

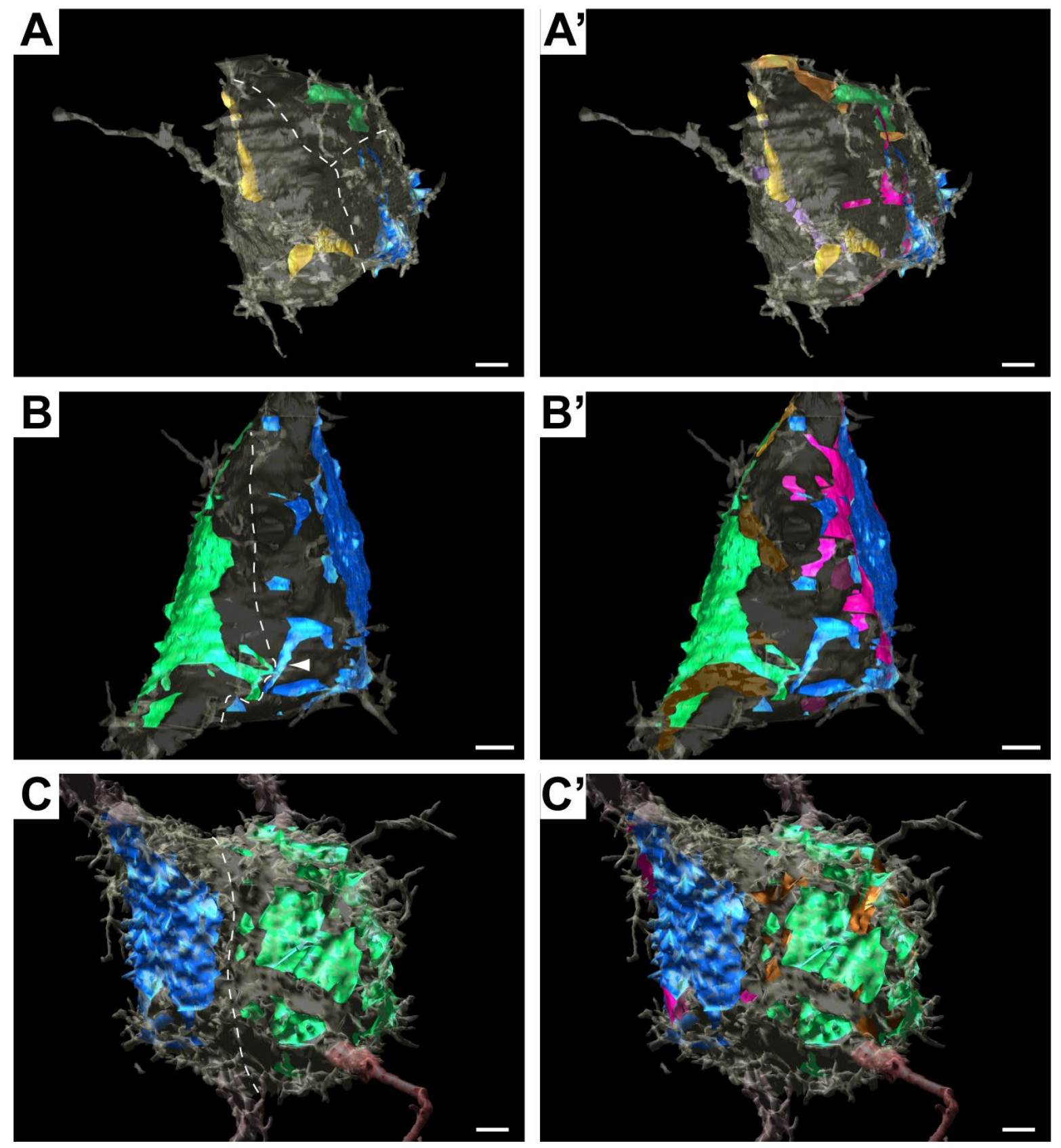

Figure 31: Competing inputs innervate distinct domains on MNTB somatic surface. A-C, Dotted lines hand drawn to illustrate well-segregated innervation territories. Inputs increase in size from panels A-C. As in other figures, largest input blue, second largest green, third largest yellow. A'-C', Inputs are segregated even after addition of glial incursions under terminals (maroon for blue terminals; bronze for green terminals; purple for yellow terminal). Cell in panel $A^{\prime}$ has green input and associated glial incursions located on opposite side of cell body which is translucent, giving the illusion of overlap with maroon glial incursion. Panels A-A', B-B', P3 cells; CC' P6 cell. Scale bars: $2 \mu \mathrm{m}$ in all panels. 
A

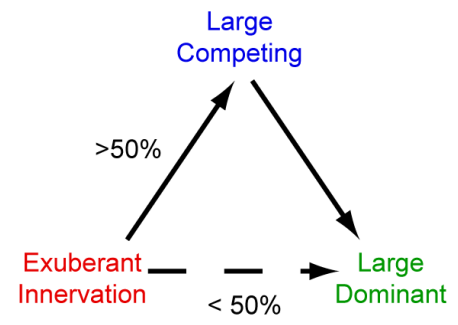

B

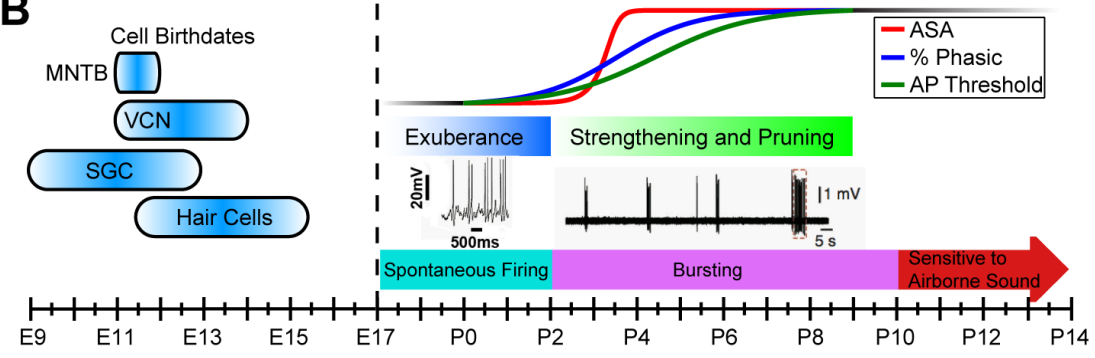

Figure 32: Summary models for dynamics of calyx growth and competition. A, Early exuberance at P2 leads to cells at P3 with multiple large inputs by strengthening and pruning to a large competing input phase in $50 \%$ of cells, or by direct emergence of dominant large calyces in $50 \%$ of cells. Since 24 -hr sampling interval may have missed competing inputs to some cells, these values are listed as minima and maxima, respectively. B, Developmental setting for calyx growth. Cells of lower auditory system born by end of second embryonic week and cochlear activity can drive contralateral MNTB at E17. Based upon data and summary from Hoffpauir et al. (2010). Transition in cochlear activity from non-bursting to bursting patterns of activity (see references in text) is proposed to trigger rapid, synchronized pre- and postsynaptic maturation in the MNTB. Exuberance of innervation by small inputs gives way to strengthening and pruning of 2-3 large inputs. Calyx growth and competition are largely resolved before the animals demonstrate sensitivity to airborne sound. 


$$
y=\theta_{1}+\frac{\theta_{2}-\theta_{1}}{1+e^{\theta_{3}^{*}\left(x-\theta_{4}\right)}}
$$

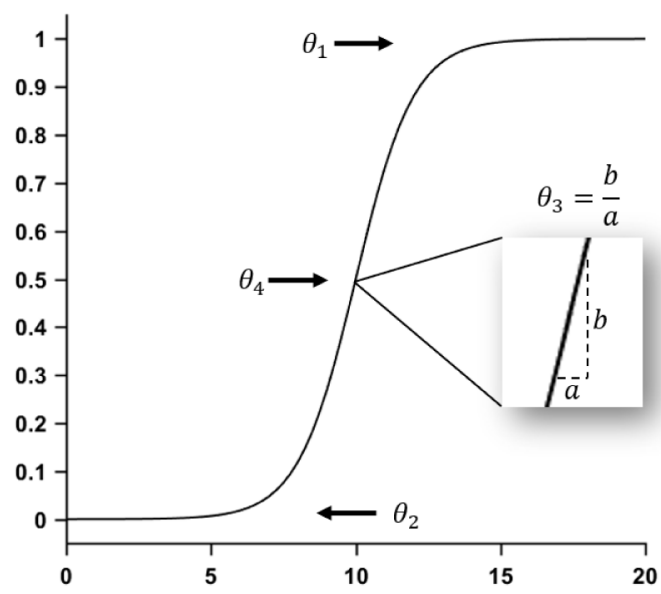

$y=\theta_{1}+\left(d_{1} * I_{1}\right)+\frac{\theta_{2}-\theta_{1}+\left(d_{2} * I_{1}\right)}{1+e^{\left(\theta_{3}+\left(d_{3} * I_{1}\right)\right) *\left(x-\theta_{4}+\left(d_{4} * I_{1}\right)\right)}}$

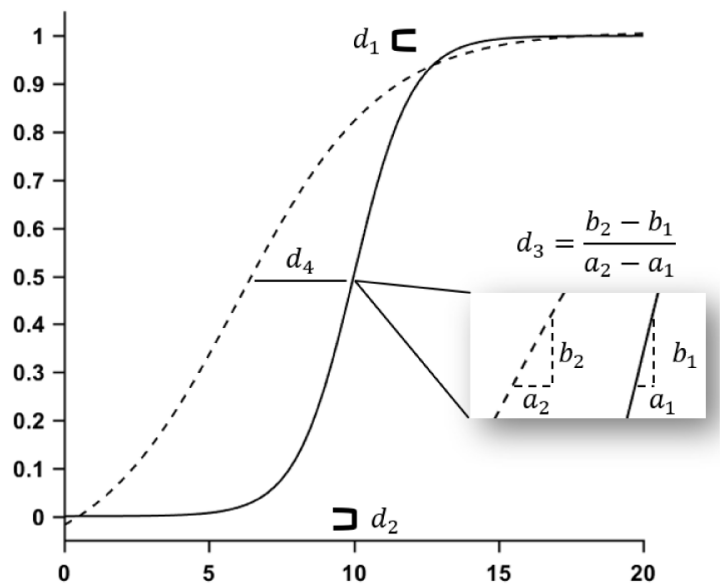

Figure 33: Logistic Curve Fitting for Comparison of Polarity Curves. Left: simple logistic curve equation (top) and graphical description (bottom). Variables are as follows: $\theta_{1}$, upper sill; $\theta_{2}$, lower sill; $\theta_{3}$, slope of curve at midpoint (b/a); $\theta_{4}$, x-location of the curve midpoint. Right: Modified logistic curve equation (top) and graphical description (bottom) for comparison between curves. Variables $d_{1}-d_{4}$ correspond to their respective $\theta$ 's and allow for these variables to be modified. $I_{1}$ is a binary value that dictates whether the $d$ variables should be used; when $I_{1}=0$, this equation reduces to the simple logistic curve. 

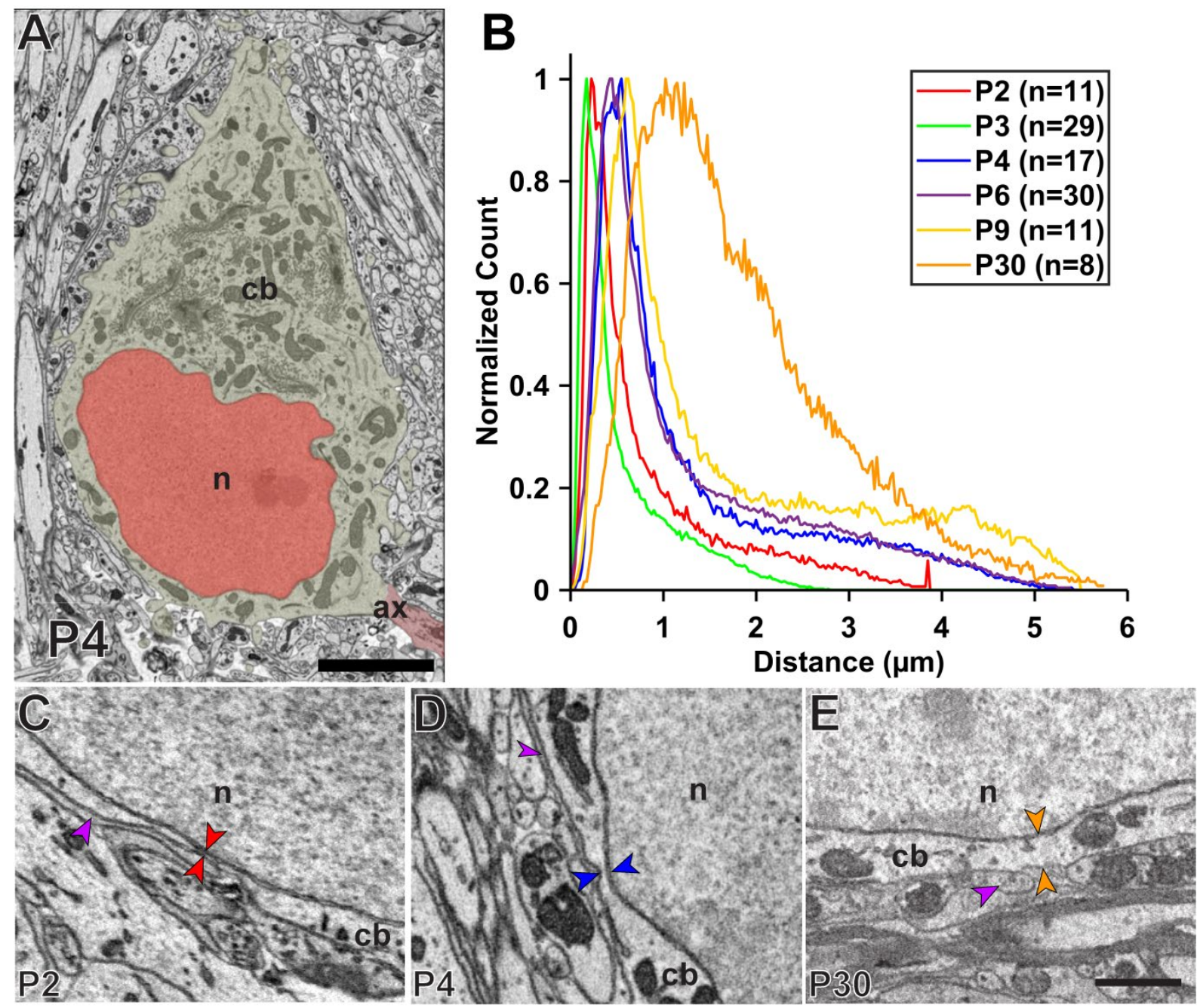

Figure 34: Principal Cell Nuclei are Eccentric. A, Electron micrograph showing a typical MNTB principal cell (cb, tan) with an eccentrically placed nucleus ( $\mathrm{n}$, red). Cell axon (ax, burgundy) is also visible. B, Graph of minimum distances from nuclear membrane to cell membrane across ages. Each curve is the average of all cells at that age. C-E, Electron micrographs showing minimum distance between cell (cb) and nuclear (n) membranes at P2 (C, red arrowheads), P4 (D, blue arrowheads), and P30 (E, orange arrowheads). Glial processes are also visible (purple arrowheads). Scale bar: $3 \mu \mathrm{m}$ for $\mathrm{A}, 1 \mu \mathrm{m}$ for $\mathrm{C}-\mathrm{E}$. 

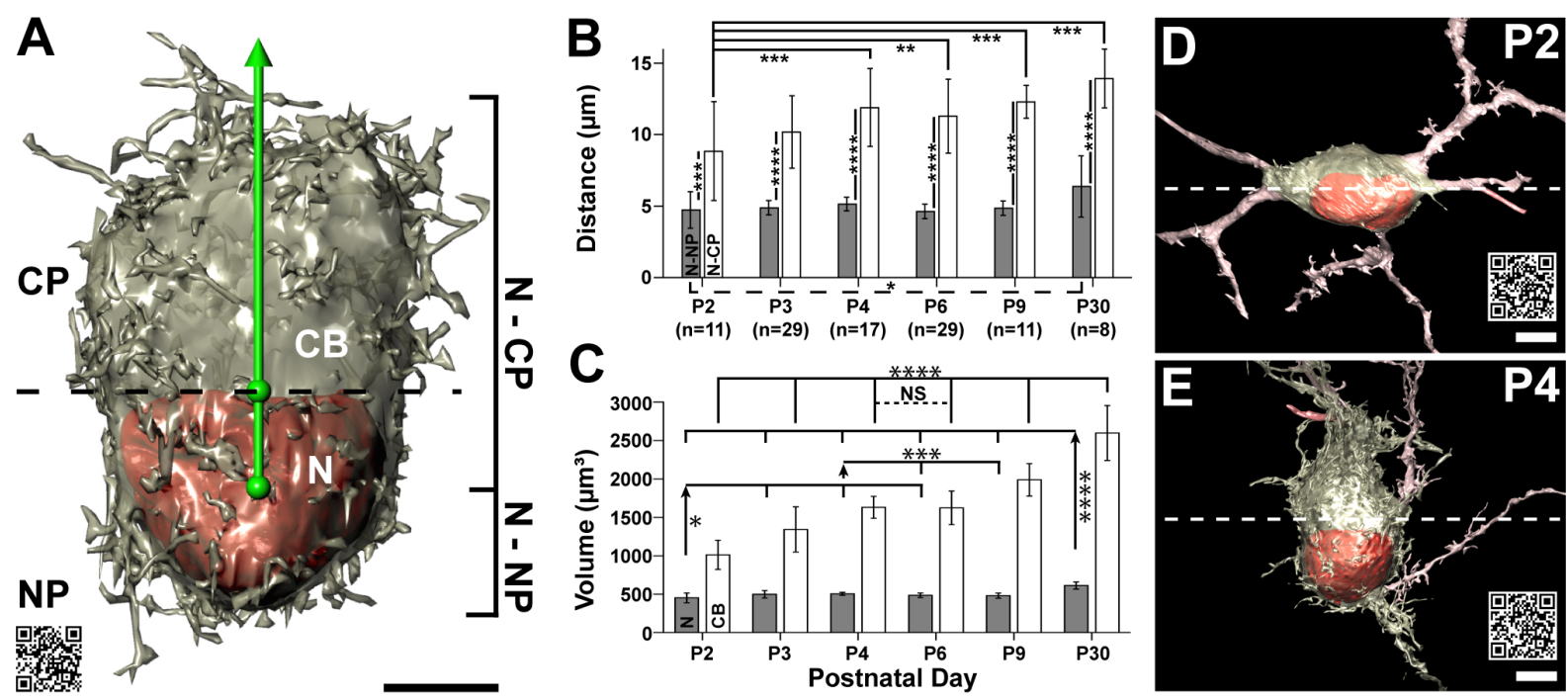

Figure 35: Quantification of Nuclear Eccentricity and Principal Cell Growth. A, Schematic representation of polarity alignment for principal cells (CB) and nuclei $(\mathrm{N})$. Centers of cell and nucleus (green spheres) were used to define a polarity axis (green arrow), and cells were bisected through the cell center to define a cytoplasmic (CP) and nuclear pole (NP). Measurement of distance from the nucleus center to the CP membrane (N-CP) or NP membrane (N-NP) along the polarity axis was used as a secondary measurement of nuclear eccentricity. B, Comparison of average N-NP and N-CP measurements across developmental time points. C, Comparison of average nucleus and cell body volumes across developmental time points. D-E, Example of transition from flattened, fusiform shape of principal cells at P2 (D) to an expanded ovoid shape (E). White dotted line represents the division between NP and CP at each age. p: $<0.05\left(^{*}\right),<0.01\left({ }^{* *}\right),<0.005\left({ }^{* *}\right),<0.001\left({ }^{* * *}\right)$; Scale bars:

$10 \mu \mathrm{m}$ for $\mathrm{A}, 5 \mu \mathrm{m}$ for $\mathrm{D}-\mathrm{E}$. 

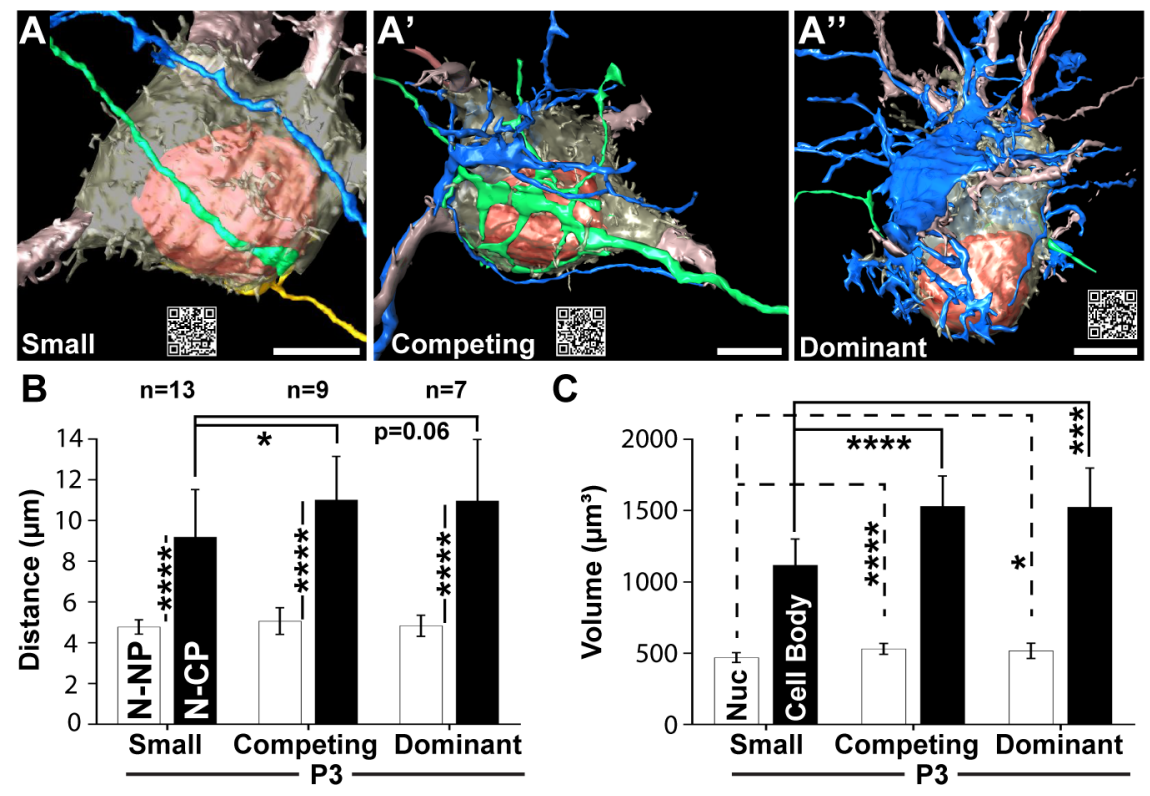

Figure 36: Nuclear Eccentricity and Principal Cell Growth by Developmental State at P3. A-A", Representative 3D reconstructions of cells in the small (A), competing ( $\left.A^{\prime}\right)$, and dominant ( $\left.A^{\prime \prime}\right)$ developmental categories. B, Comparison of average N-NP and N-CP distances by developmental stage. C, Comparison of average nucleus and cell body volumes by developmental stage. p: $<0.05\left(^{*}\right),<0.005\left(^{* * *}\right),<0.001\left(^{* * * *}\right)$ Scale bar: $5 \mu \mathrm{m}$ for A-A". 

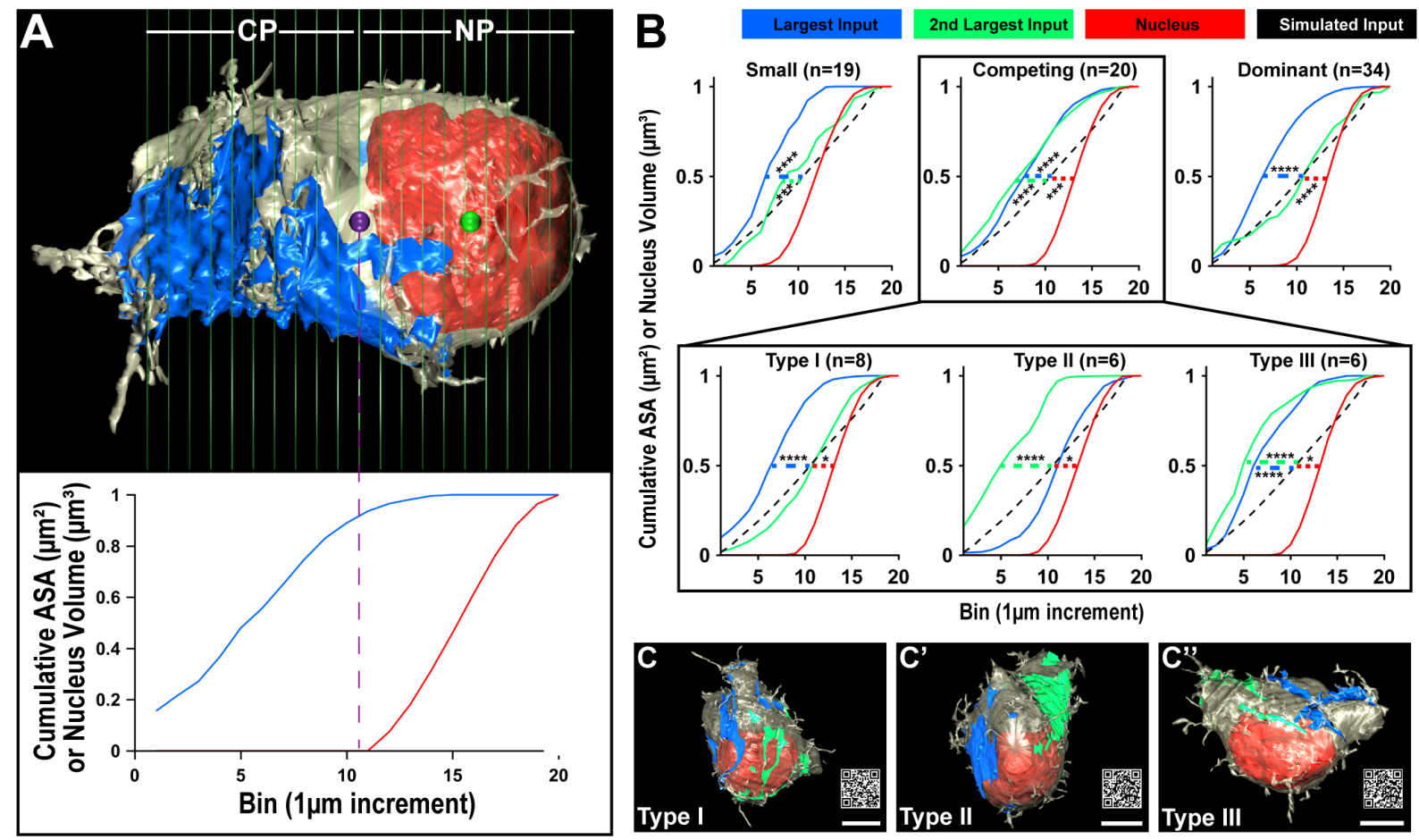

Figure 37: Calyceal Terminals are Polarized with Respect to the Nucleus. A, Schematic of measurement technique for assessing terminal polarity. Cell body (tan), nucleus (red), and terminals (blue) were divided into $1 \mu \mathrm{m}$ bins (green lines) and surface area of terminals or volume of nuclei were accumulated from the leftmost bin in the cytoplasmic pole (CP) to the rightmost bin in the nuclear pole (NP) to produce cumulative curves (lower). Results were assessed based on the CP-NP division (purple dashed line) for overall polarity. Purple and green spheres represent cell and nucleus centers, respectively. B, Top: Comparison of average polarity curves to simulated random terminal placement (black dashed line) for the two largest terminals on each cell (blue and green, respectively) in each developmental category, as well as nucleus location (red). Bottom: Competing polarity curves were further subdivided into three types based on $k$-means clustering results. C-C', Representative 3D reconstructions of Type I (C), Type II (C'), and Type III (C') competing cells (tan) with nuclei (red) and largest and second largest terminals (blue and green, respectively). p: $<0.05\left(^{*}\right),<0.005\left(^{* * *}\right),<0.001$ $\left.{ }^{* * * *}\right)$; Scale bar: $5 \mu \mathrm{m}$ for C-C". 


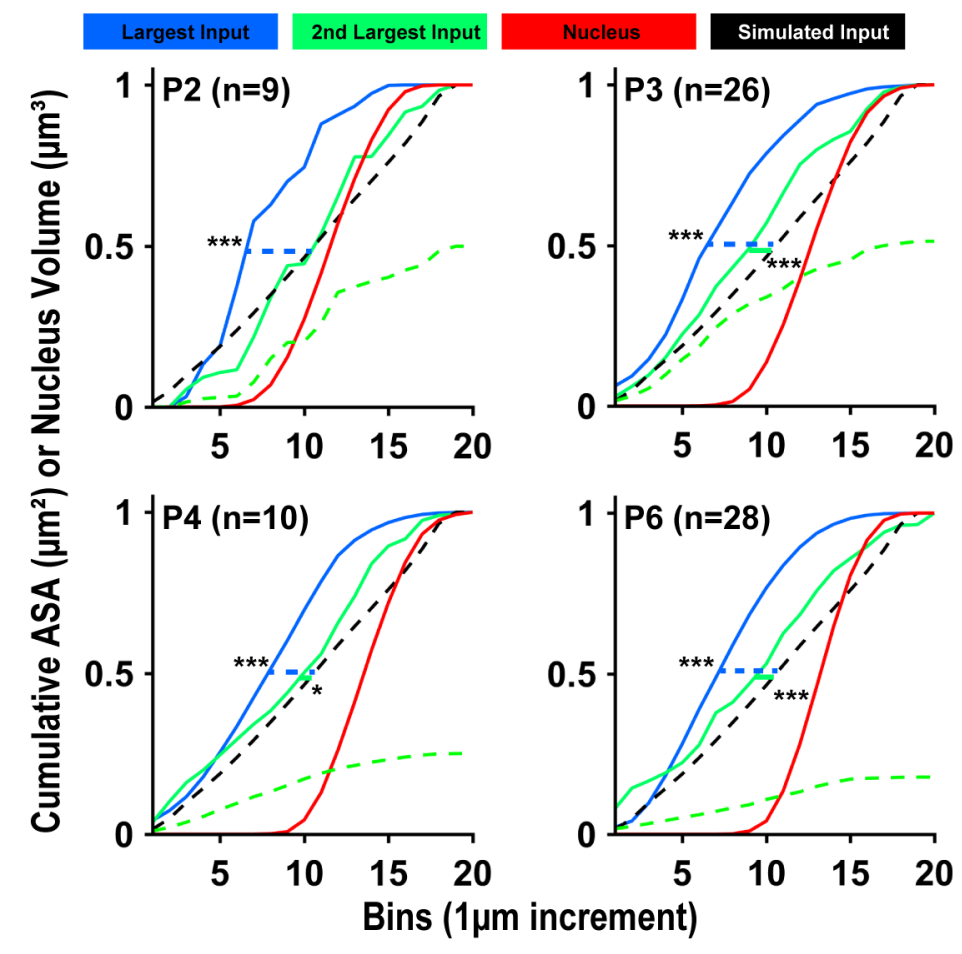

Figure 38: Polarity Curves by Postnatal Day. Comparison of average terminal polarity curves by postnatal day. Green dashed line represents the polarity curve for the second largest input scaled by the ratio of input sizes $\left.(I 2 / 11) . p:<0.05\left(^{*}\right),<0.005^{(* *}\right)$. 


\section{P3 Only}

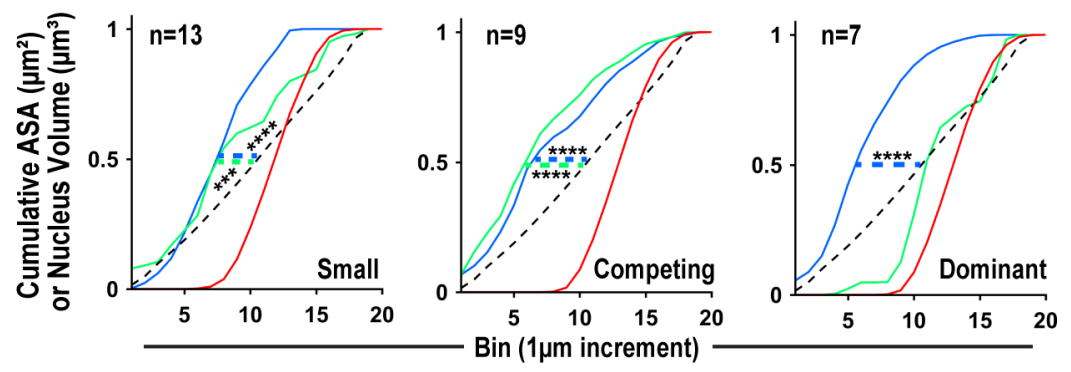

Figure 39: Polarity Curves by Developmental State (P3 Only). Comparison of polarity curves for developmental state confined to postnatal day 3. p: $\left.<0.005\left(^{(* \star}\right),<0.001{ }^{* \star *}\right)$. 

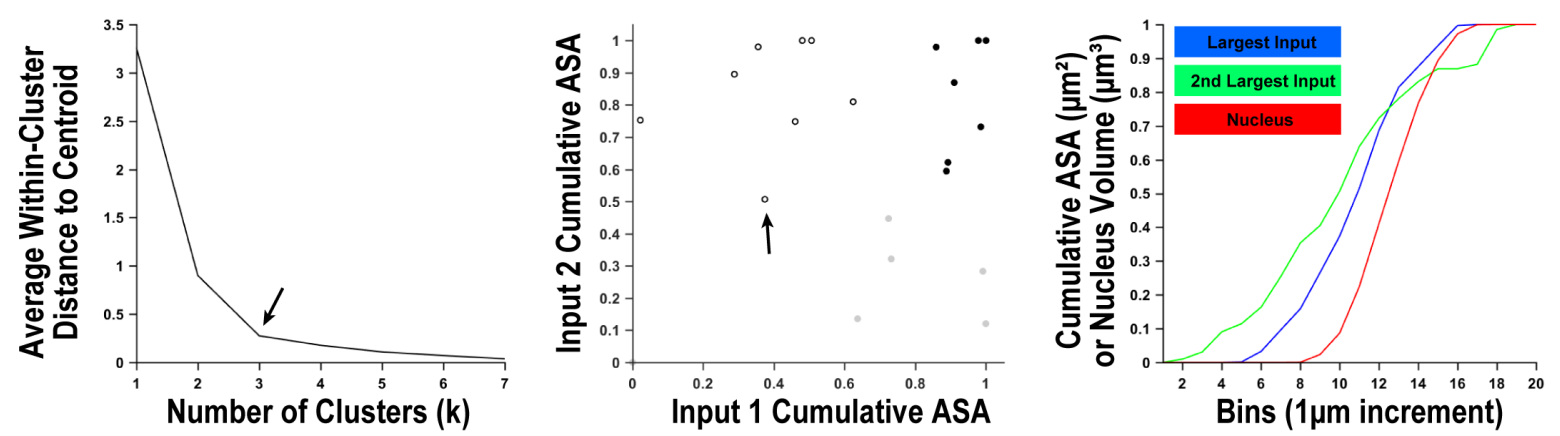

Figure 40: Competing Subtype K-Means Clustering Analysis. Left: Optimal $k$-means cluster determination for competitive subtypes based on inflection point of curve (arrow) comparing number of clusters $(x)$ and the average within-cluster distance to the group centroid $(\mathrm{y})$. Center: Results of $k$-means clustering of competitive subtypes. Arrow shows outlier that could potentially be sorted into two groups. Right: Terminal polarity curves for outlier (arrow in center graph). Curves show trend toward antipolar placement of terminals. 


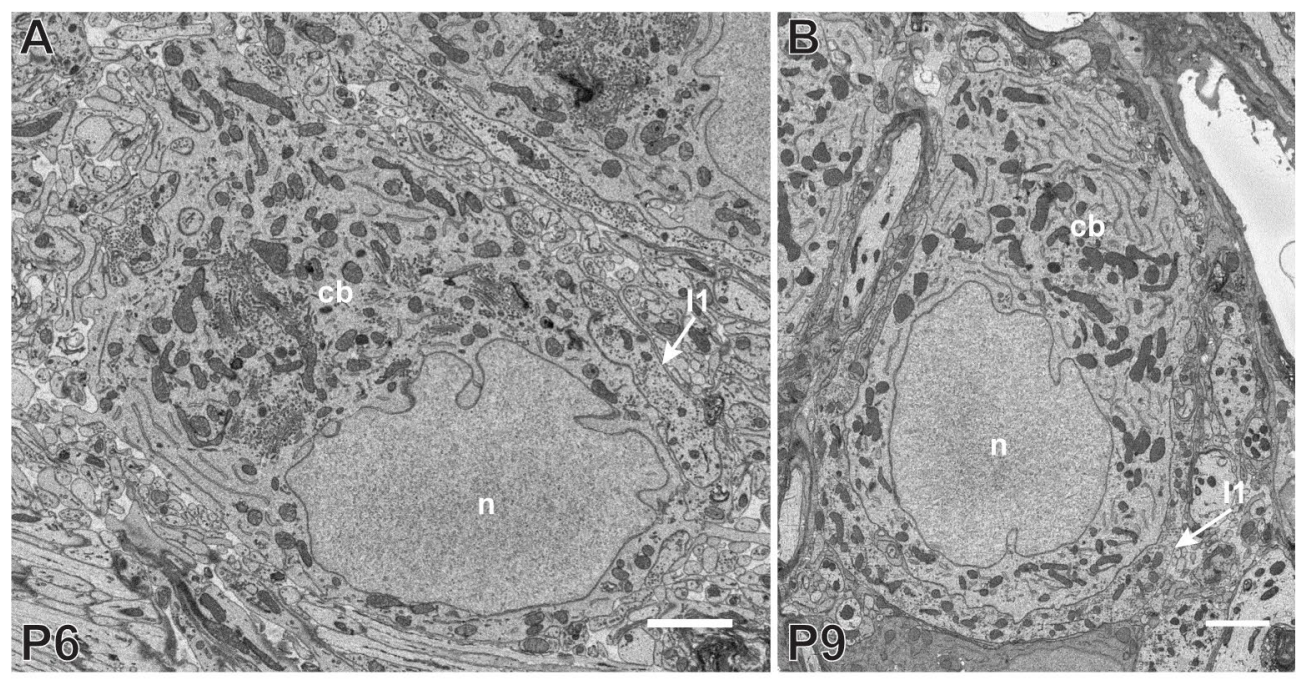

Figure 41: Antipolar Calyces in Later Postnatal Development. Electron micrographs of principal cells (cb) at postnatal days 6 (A) and 9 (B). A large input for each cell (I1, arrow) can be seen closely apposed to the eccentric nucleus $(\mathrm{n})$. Scale bar: $2 \mu \mathrm{m}$. 

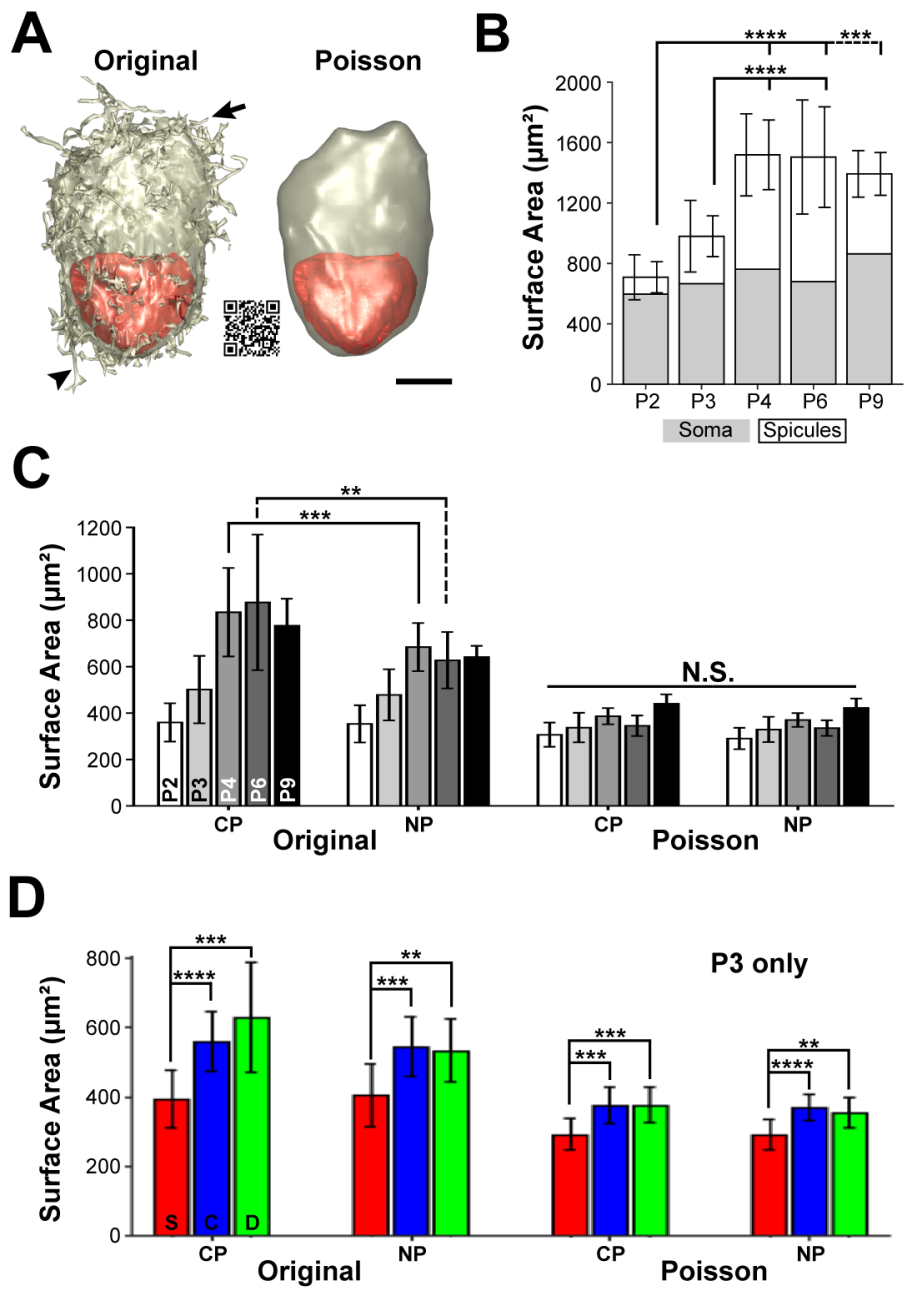

Figure 42: Surface Area Expansion and Somatic Spicules. A, Comparison of original cell reconstruction with somatic spicules and Poisson reconstruction of the same cell. Small somatic spicules (arrow) and longer cellular processes (arrowhead) can be seen on the original cell but are absent on the Poisson reconstruction. B, Comparison of surface areas between original (full bars) and Poisson ("soma", grey bars) reconstructions. The difference ("Spicules", white bars) represent an approximate measurement of surface area found on somatic spicules. C, Pairwise comparison of surface area between the CP and NP of original and Poisson reconstructions at P2-P9. D, Comparison of surface areas for CP or NP between developmental states at P3 (small - S, red; competing $-\mathrm{C}$, blue; dominant $-\mathrm{D}$, green) for original and Poisson reconstructions. p: $<0.01\left(^{(*}\right),<0.005\left(^{* * *}\right)$, $<0.0011^{(* * *)}$; Scale bar: $5 \mu \mathrm{m}$ for $\mathrm{A}$. 

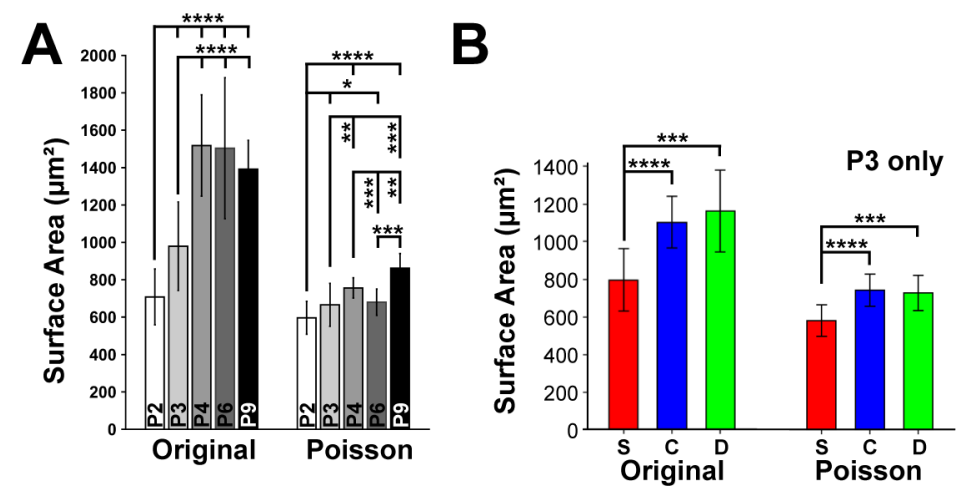

Figure 43: Average Total Surface Areas by Postnatal Day or Developmental State (P3). A, Comparison of average surface areas for each postnatal day for either original reconstructions (left) or Poisson reconstructions (right). B, Comparison of average surface areas for developmental state confined to postnatal day 3 for either original reconstructions (left) or Poisson reconstructions (right). p: $<0.05\left({ }^{*}\right),<0.01\left(^{(*}\right),<0.005\left({ }^{* * *}\right),<0.001\left(^{* * * *}\right)$. 


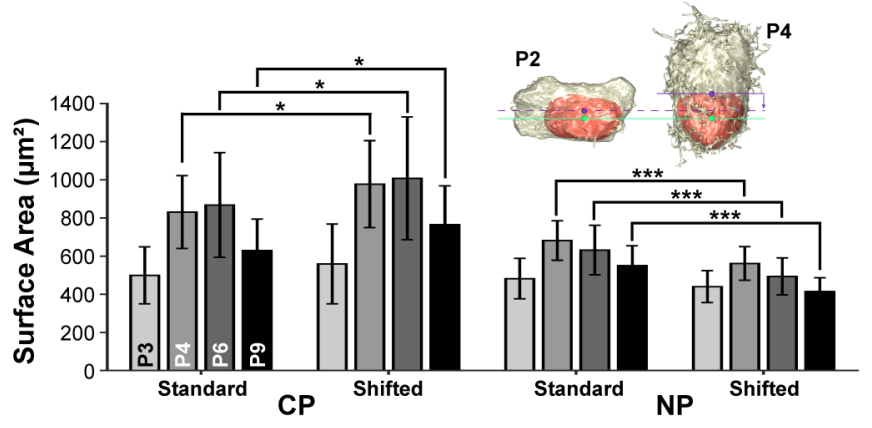

Figure 44: Shift in Bisector Plane Defining Cytoplasmic and Nuclear Poles. If polarity is established early in development ( $\angle P 2)$, it is possible that the early division between CP and NP (purple dashed line) is maintained throughout development, despite the shift of the cell center during growth (purple spheres). To determine the impact this would have on our morphological assessments, bisector planes at later ages (purple solid line) were shifted to the average distance between the P2 nucleus and cell centers (purple arrow). As expected, a significant shift in surface area toward the CP and away from the NP is evident at P4-P9. If asymmetry in the principal cell is established and maintained early on, the results of this study would be further strengthened. $\mathrm{p}$ : $<0.05\left(^{*}\right),<0.005\left({ }^{* * *}\right)$. 

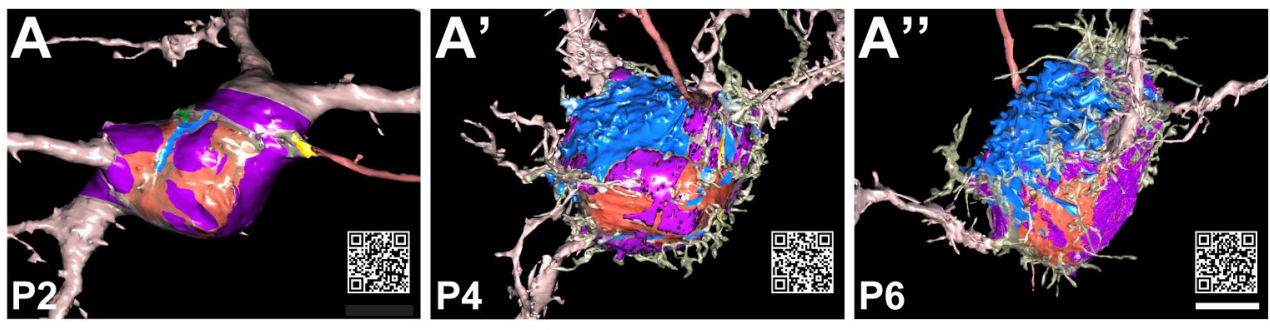

B

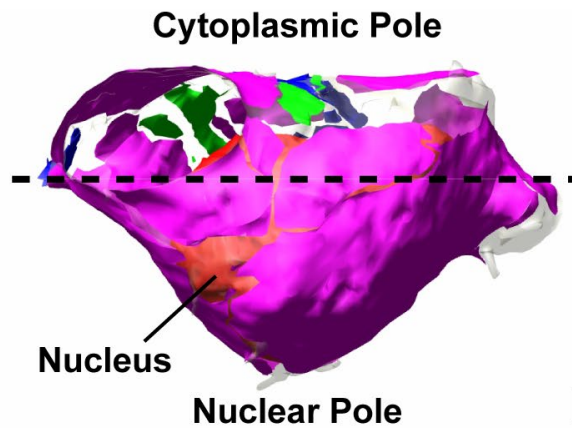

P2

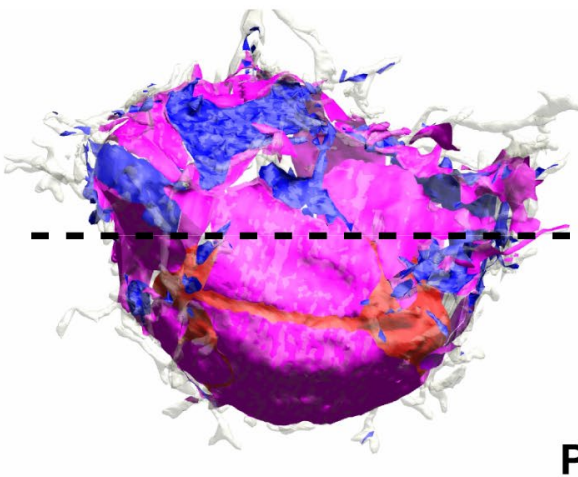

P4
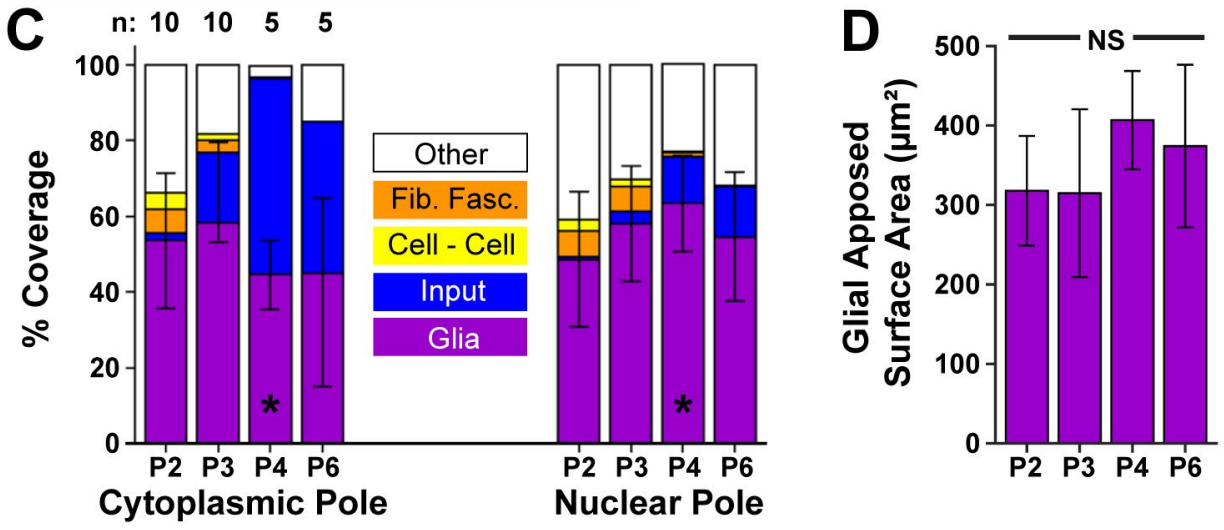

Figure 45: Glial Process Coverage of Principal Cells. A-A", Representative 3D reconstructions of MNTB principal cells (tan, transparent) with nuclei (red), dendrites (brown), axons (burgundy), terminals (blue, green, gold) and glial coverage (purple) for P2 (A), P4 (A'), and P6 (A"). B, 3D reconstructions from A-A" without processes to demonstrate measurement process. Black dashed lines represent bisection plane dividing $C P$ and NP. Color scheme is the same as A-A". C, Breakdown of average surface coverage of MNTB principal cells on either CP or NP by source (glia - purple, inputs - blue, cell-cell contact - yellow, fiber fascicile - orange) for P2P6. "Other" represents either extracellular space or small processes not clearly belonging to another category. Two asterisks represent statistical difference between glial coverage for P4 only. Error bars are for glial coverage only. D, Glial coverage for whole cells averaged for each postnatal day (P2-P6). p: $<0.05\left(^{*}\right)$ Scale bar: $5 \mu \mathrm{m}$ for A-A". 


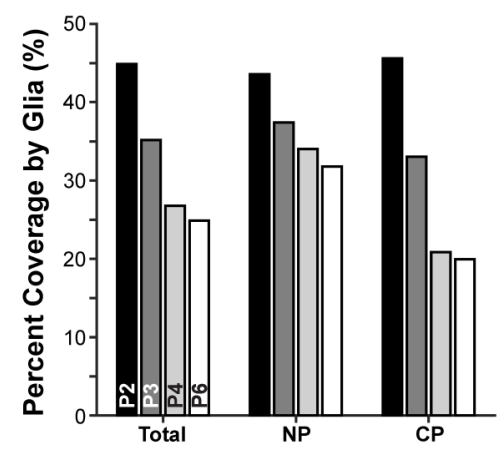

Figure 46: Percentage of Glial Coverage of Cell Surface with Spicules. When glial coverage is considered with regards to cells with somatic spicules, the percentage of surface coverage decreases across postnatal days (P2-P6). This decrease is gradual in regard to the NP, but precipitous for the CP. 

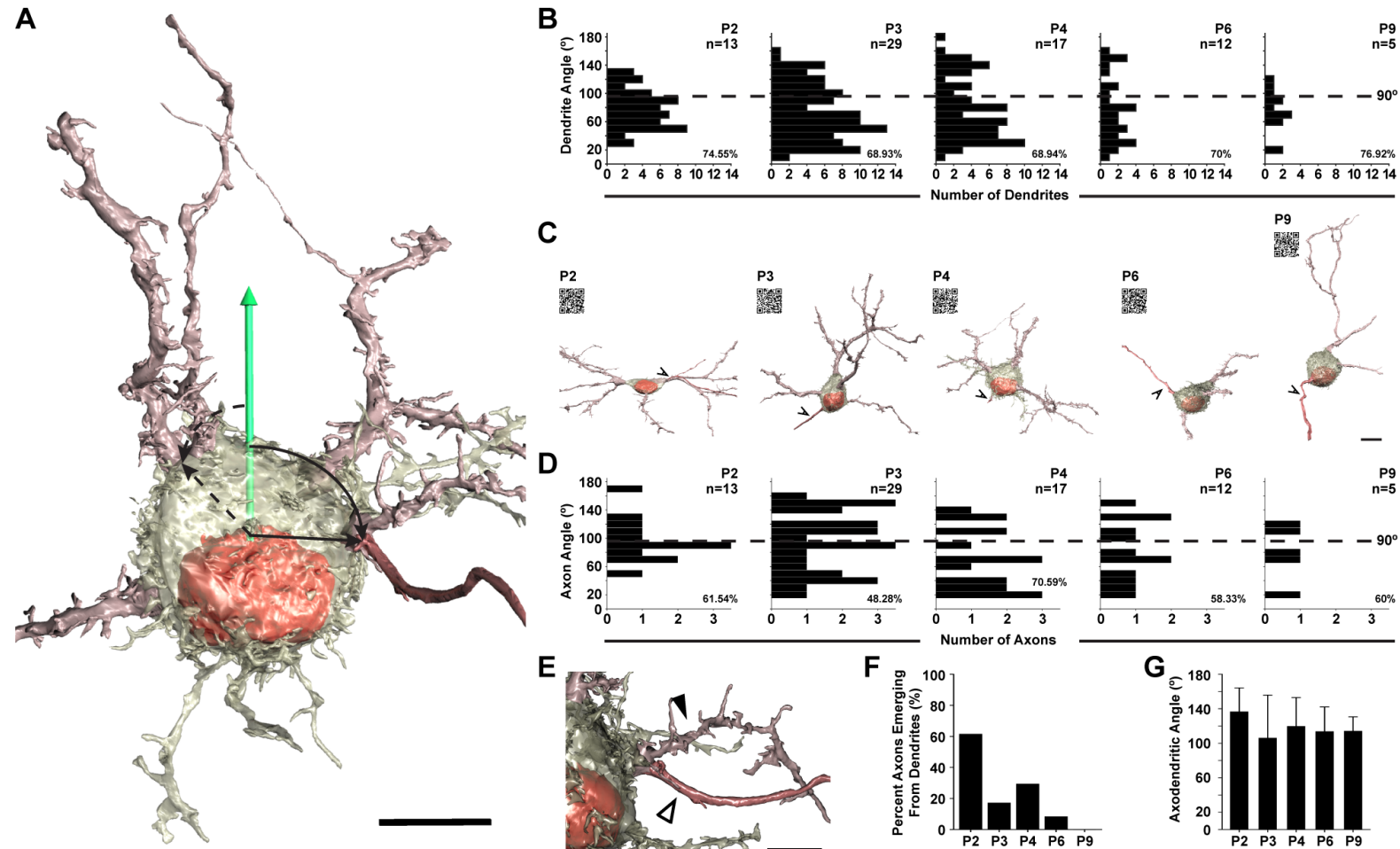

C
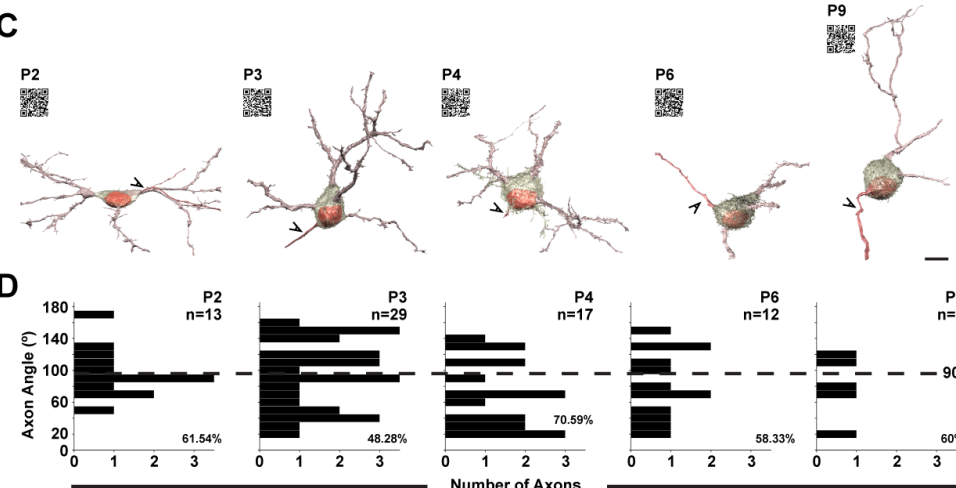

E

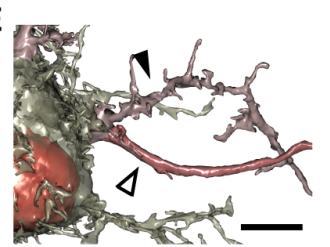

$\mathbf{F}$

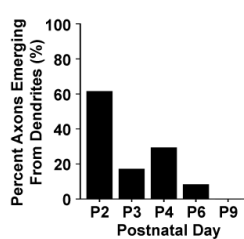

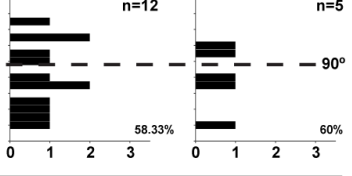

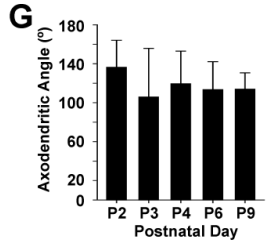

Figure 47: Dendrite Angles with Respect to Polarity Axis. A, Schematic representation of dendrite solid angle (dotted line and arc) and axon solid angle (solid line and arc) measured from the polarity vector (green arrow). Cell body (tan), nucleus (red), dendrites (brown), and axon (burgundy) also depicted. B, Count of dendrites by angle from the polarity vector for postnatal days P2-P9 binned at $10^{\circ}$ increments. Black dotted line divides the CP $\left(0-90^{\circ}\right)$ and NP $\left(90-180^{\circ}\right)$. Percentages are number of dendrites located on the CP compared to all dendrites at that age. B, Montage of typical principal cell dendrite morphology by postnatal day, including cell bodies (tan) nuclei (red), dendrites (brown), and axons (burgundy, open arrowhead). C, Axon angle measurements for postnatal days P2-P9 performed as in A. D, 3D reconstruction of axon (burgundy, open arrowhead) emerging from a dendrite (brown, filled arrowhead). Cell body (tan) and nucleus (red) are also visible. E, Quantification of axons emerging from a dendrite by postnatal day, P2-P9. F, Angle between the axon and average dendrite location measured with reference to the cell center averaged over all cells at each postnatal day, P2-P9. Scale bar: $10 \mu \mathrm{m}$ for $\mathrm{B}, 5 \mu \mathrm{m}$ for $\mathrm{D}$. 

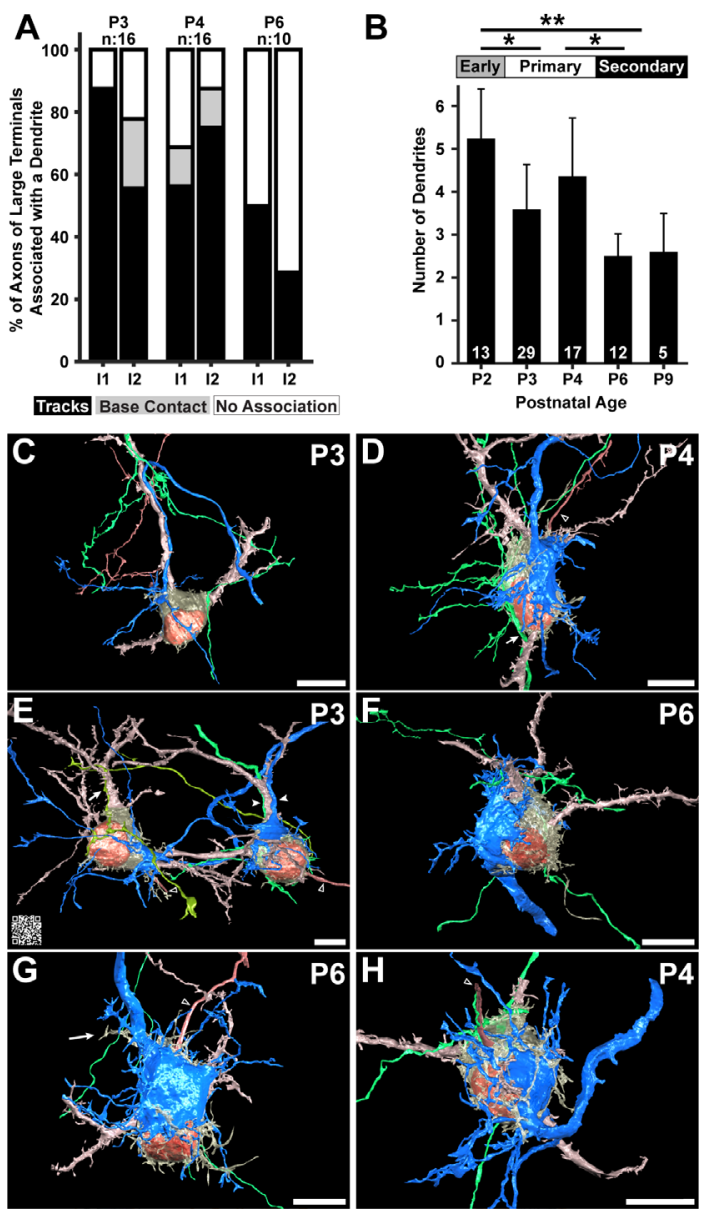

Figure 48: Axodendritic Association and Dendritic Pruning. A, Qualitative assessment of association between calycigenic axons for the two largest inputs per cell $(11,12)$ and principal cell dendrites for competing and dominant cells combined. Axons were classified as either tracking along a dendrite to the soma ("Tracks", black), touching down on the somatic surface at the base of a dendrite ("Base Contact", grey), or not associating with a dendrite ("No Association", white). B, Average number of dendrites per cell at each postnatal age (P2-P9). Two significant decreases in dendrite number were found, dividing cells into three stages based on dendrite number: Early (5+ dendrites), Primary (3-4 dendrites), and Secondary (2 or fewer dendrites). Significance bar at top compares Early and Secondary dendrite numbers. C-H, Representative 3D reconstructions of axodendritic associations including cell bodies (tan, translucent), nuclei (red), dendrites (brown), axons (burgundy, open arrowhead) and two largest inputs (I1, blue and I2, green, respectively). C, I1 axon (blue) tracking a dendrite to the soma. 12 axon touches down at a dendrite base (arrow). D, Dendrite base contact for I1 on CP (arrowhead) and $\mathrm{I} 2$ on nuclear pole (arrow). E, Exceptional case of dual calyces. I1 axon (blue) bifurcates after contacting dendrite (asterisk). One branch tracks along the same dendrite as 12 to the soma on the right (opposing arrowheads) to form a large terminal. The second II branch contacts the dendrite of a neighboring cell (center arrowhead) and tracks to the soma to form another large terminal. A third axon (I2 for the left cell, olive) tracks down a third dendrite (left arrowhead) to the left cell soma. F, Large I1 axon contacts the principal cell on the NP without association with a dendrite. G, Large I1 axon contacts the principal cell on the CP without apparent association with a dendrite. However, a large somatic process (arrow) is directly apposed to the axon, possibly representing a degenerating dendrite. $\mathbf{H}$, Large $\mathrm{I} 1$ axon touches down at the base of a dendrite on the NP and grows almost exclusively on the NP (antipolar calyx). Scale bar: $10 \mu \mathrm{m}$ for $\mathrm{C}-\mathrm{H}$. 


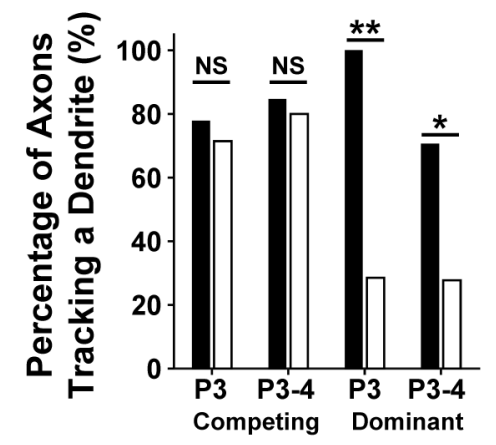

Figure 49: Comparison of Axodendritic Association for Competing and Dominant Developmental Categories. Axodendritic association was assessed specifically for P3-P4 cells ("primary" dendritic stage) and compared between competing and dominant developmental categories. While no significant difference in axodendritic association was found for terminals in active competition, the axon of the second largest terminal in dominant cases was significantly less likely to be associated with a dendrite. p: $<0.05\left({ }^{*}\right),<0.01\left(^{(*)}\right.$. 


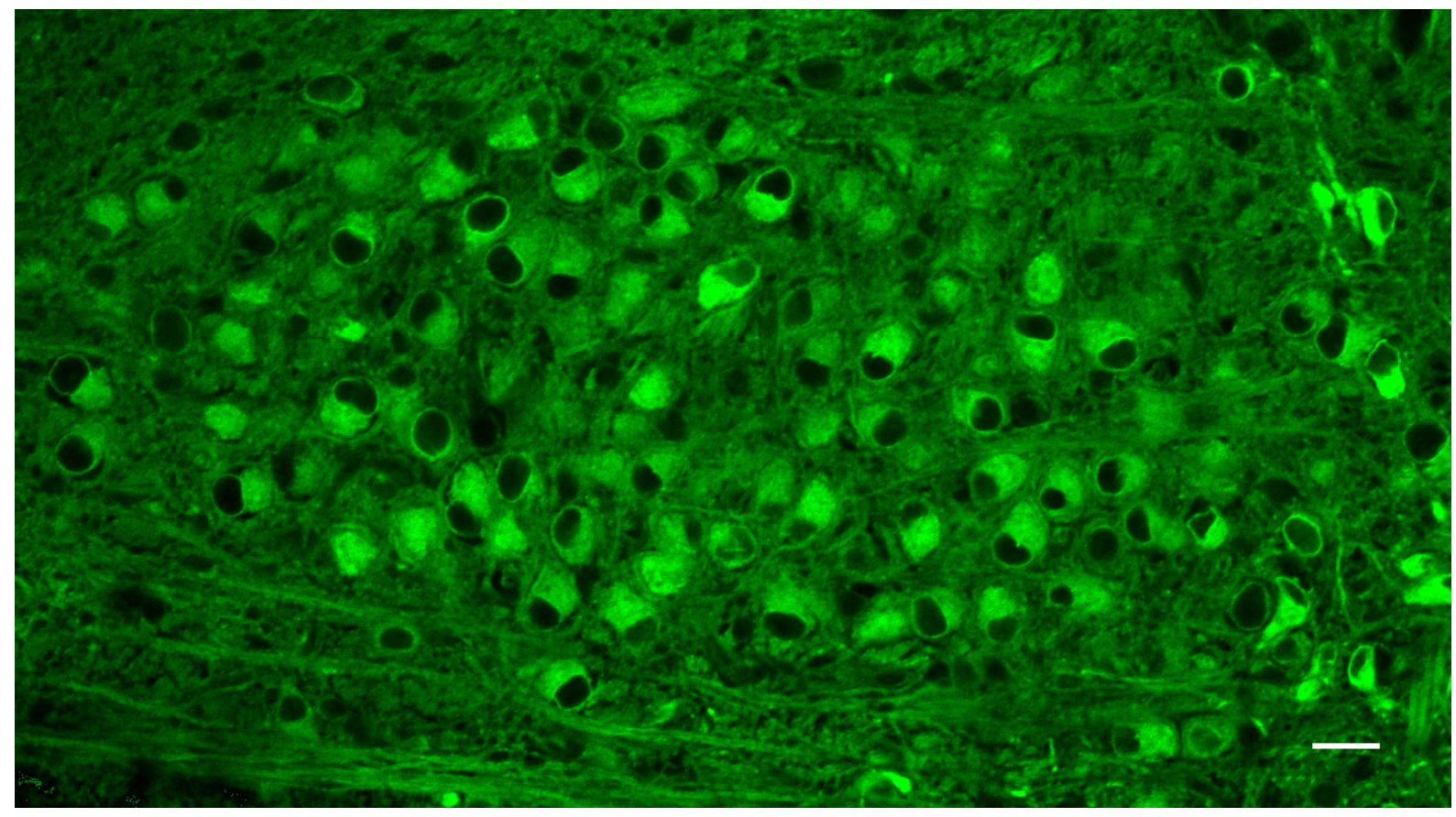

Figure 50: MNTB of KASH/Engrailed-Cre Overexpression Mouse at P6. Overexpression of the KASH domain of the LINC complex in MNTB principal cells using Engrailed-Cre recombination caused no change in nuclear location. Green label is the GFP-tagged overexpressed KASH construct. Scale bar: $20 \mu \mathrm{m}$. 
Table 1. Image Volumes and Analysis

\begin{tabular}{cccccc}
\hline $\begin{array}{c}\text { Postnatal } \\
\text { Day }\end{array}$ & $\begin{array}{c}\text { Tissue } \\
\text { Dimensions }(\boldsymbol{\mu m})\end{array}$ & $\begin{array}{c}\text { Pixel } \\
\text { Size } \\
(\mathbf{n m})\end{array}$ & $\begin{array}{c}\text { Tissue } \\
\text { Volume } \\
\left(\boldsymbol{\mu m}^{3}\right)\end{array}$ & $\begin{array}{c}\text { Number of } \\
\text { Cells } \\
\text { Analyzed }\end{array}$ & $\begin{array}{c}\text { Number of } \\
\text { Cells in } \\
\text { Volume }\end{array}$ \\
\hline 2 & $124.8 \times 90.8 \times 85.2$ & 5.7 & 965,473 & 21 & 71 \\
\hline 3 & $96 \times 84 \times 50.2$ & 12.0 & 404,813 & 37 & 37 \\
\hline 4 & $80 \times 80 \times 59.9$ & 10.0 & 383,360 & 18 & 18 \\
\hline 6 & $98 \times 78.4 \times 68$ & 4.9 & 522,458 & 43 & 43 \\
\hline 9 & $\begin{array}{c}153.6 \times 115.2 \times \\
59.2\end{array}$ & 4.8 & $1,047,527$ & 66 & 66 \\
\hline \hline
\end{tabular}




\section{APPENDIX}

I.

Permission for use of article in Gissertation

Jn permiselons to me

5.21 PM

Dear Dr. Holcomb,

Thank you for your email. Permission is granted to reproduce the requested material listed below with No fee in print and electronic format for use in your doctoral thesis/dissertation. Please contact me if you have any questions or if you need another form of permission.

Regards,

SfN Central Office

From: Foul Holcomb < pul holcombersmall coms

Sent: Mondsw June 11, 2018 4:19 PM

To: in permissions

Subject: Permission for use of article in dissertation

To whom it may concern:

My name is Paul Holcomb, and I published an article in your journal that I would like to regroduce in my dissertation. Here is the reference:

Holcomb PS, Hoffpauir BK, Hoyson MC, Jackson DR, Deerinck TJ, Marrs GS, Dehaff M, Wu J, Elisman MH, Spirou GA. "Synaptic Inputs Compete During Rapid Formation of the Calyx of Held: A New Model System for Neural Development". J Neurosci. 2013 Aug 7;33(32):12954-69. doé: 10.1523/JNEUROSCI.1087-13.2013.

I would appreciate any assistance you could provide in this matter.

Sincerely.

Paul Holcomb 